Supporting Information

\title{
Cascade Oxidative Coupling/Cyclization: A Gateway to 3-Amino Polysubstituted Five-Membered Heterocycles
}

\author{
Kaizhi Li and Jingsong You* \\ Key Laboratory of Green Chemistry and Technology of Ministry of Education, \\ College of Chemistry, and State Key Laboratory of Biotherapy, West China Hospital, \\ West China Medical School, Sichuan University, 29 Wangjiang Road, Chengdu \\ 610064, China \\ Email: jsyou@scu.edu.cn
}

\section{Table of contents}

I. X-Ray crystal structure and data for compound 31 ..........................................

II. X-Ray crystal structure and data for compound $\mathbf{4 a}$........................................ 7

III. X-Ray crystal structure and data for compound 5a.......................................... 12

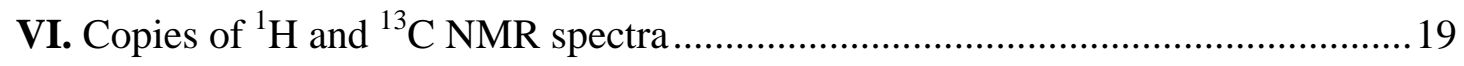




\section{X-Ray crystal structure and data for compound 3I (CCDC-1404100)}

X-Ray single-crystal diffraction data of crystals $3 \mathbf{l}$ was collected by using $\mathrm{Cu} \mathrm{K} \alpha$ radiation. The crystal was kept at $293.15 \mathrm{~K}$ during data collection. Using Olex2, the structure was solved with the Superflip structure solution program using Charge Flipping and refined with the ShelXL refinement package using Least Squares minimisation.
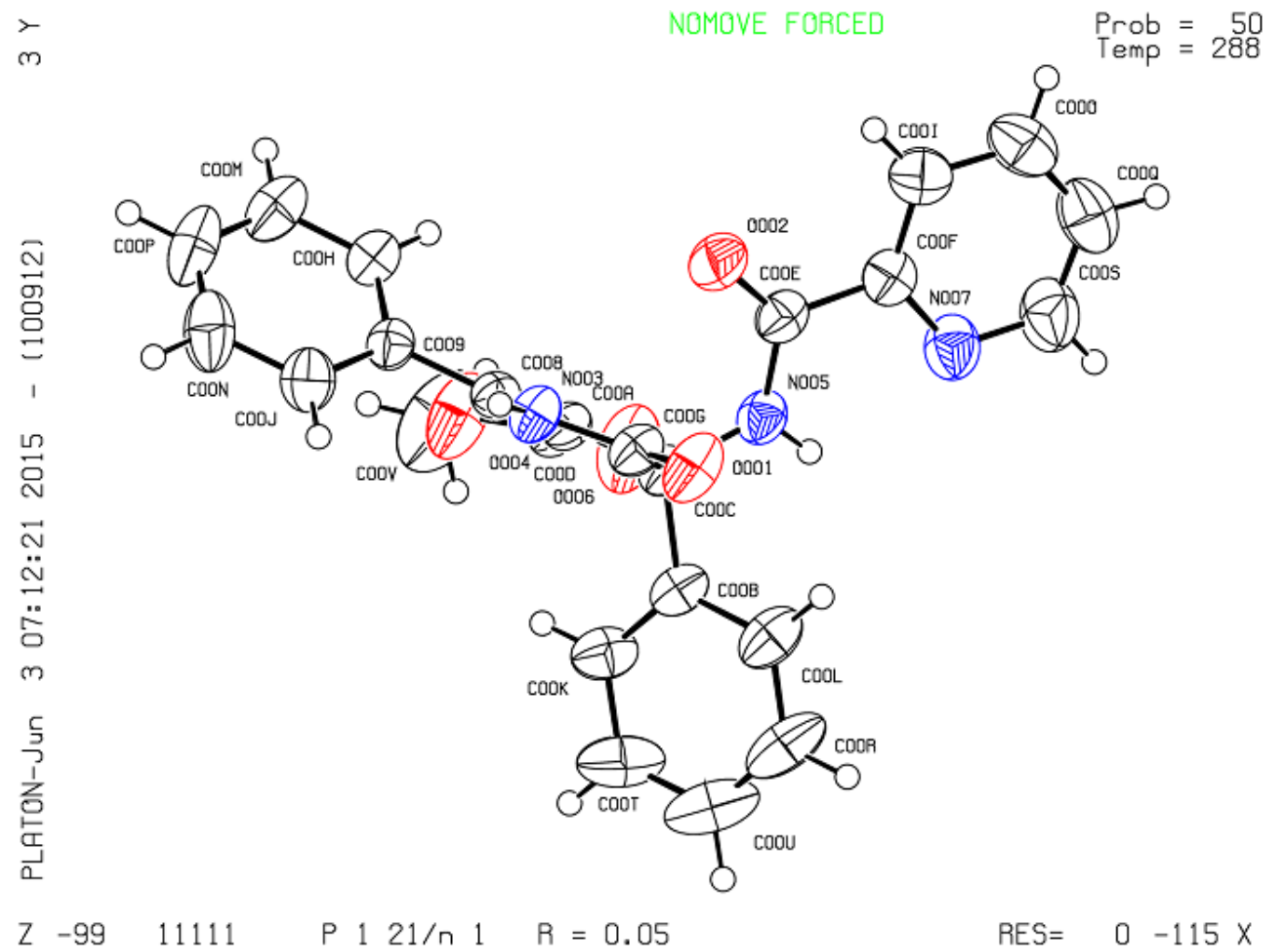

Figure S1 Crystal structure of $\mathbf{3 b}$ (The ellipsoid contour probability level is $\mathbf{5 0 \%}$ )

Table S1. Crystal data and structure refinement for 31

\begin{tabular}{|c|c|}
\hline Identification code & 31 \\
\hline Empirical formula & $\mathrm{C}_{24} \mathrm{H}_{19} \mathrm{~N}_{3} \mathrm{O}_{4}$ \\
\hline Formula weight & 413.42 \\
\hline Temperature/K & $288.42(10)$ \\
\hline Crystal system & monoclinic \\
\hline Space group & $\mathrm{P} 2{ }_{1} / \mathrm{n}$ \\
\hline $\mathrm{a} / \AA$ & $9.9179(4)$ \\
\hline $\mathrm{b} / \AA$ & $24.4164(6)$ \\
\hline $\mathrm{c} / \AA$ & $9.9702(4)$ \\
\hline$\alpha /^{\circ}$ & 90 \\
\hline
\end{tabular}




\begin{tabular}{|c|c|}
\hline$\beta /{ }^{\circ}$ & $118.045(5)$ \\
\hline$\gamma /{ }^{\circ}$ & 90 \\
\hline Volume $/ \AA^{3}$ & $2130.87(15)$ \\
\hline $\mathrm{Z}$ & 4 \\
\hline$\rho_{\text {calc }} \mathrm{g} / \mathrm{cm}^{3}$ & 1.289 \\
\hline$\mu / \mathrm{mm}^{-1}$ & 0.089 \\
\hline $\mathrm{F}(000)$ & 864.0 \\
\hline Crystal size $/ \mathrm{mm}^{3}$ & $0.4 \times 0.3 \times 0.2$ \\
\hline Radiation & $\operatorname{MoK} \alpha(\lambda=0.71073)$ \\
\hline $2 \Theta$ range for data collection $/{ }^{\circ}$ & 6.82 to 52.744 \\
\hline Index ranges & $-12 \leq \mathrm{h} \leq 12,-30 \leq \mathrm{k} \leq 30,-12 \leq 1 \leq 12$ \\
\hline Reflections collected & 24808 \\
\hline Independent reflections & $4359\left[\mathrm{R}_{\text {int }}=0.0389, \mathrm{R}_{\text {sigma }}=0.0257\right]$ \\
\hline Data/restraints/parameters & $4359 / 0 / 281$ \\
\hline Goodness-of-fit on $\mathrm{F}^{2}$ & 1.024 \\
\hline Final $R$ indexes $[\mathrm{I}>=2 \sigma(\mathrm{I})]$ & $\mathrm{R}_{1}=0.0482, \mathrm{wR}_{2}=0.1029$ \\
\hline Final $\mathrm{R}$ indexes [all data] & $\mathrm{R}_{1}=0.0696, \mathrm{wR}_{2}=0.1138$ \\
\hline Largest diff. peak/hole / e $\AA^{-3}$ & $0.19 /-0.19$ \\
\hline
\end{tabular}

Table S2 Fractional Atomic Coordinates $\left(\times 10^{4}\right)$ and Equivalent Isotropic Displacement Parameters $\left(\AA^{2} \times 10^{3}\right)$ for 31 . $U_{\text {eq }}$ is defined as $1 / 3$ of of the trace of the orthogonalised $U_{\text {IJ }}$ tensor.

\begin{tabular}{lcccc}
\hline Atom & $x$ & $y$ & $z$ & $\mathrm{U}(\mathrm{eq})$ \\
\hline O001 & $4963.9(17)$ & $4922.6(5)$ & $1791.1(15)$ & $58.4(4)$ \\
O002 & $8183.6(16)$ & $4308.6(6)$ & $3453.4(15)$ & $61.4(4)$ \\
N003 & $5191.4(16)$ & $4237.2(5)$ & $332.6(16)$ & $43.3(3)$ \\
O004 & $5537(2)$ & $2548.0(5)$ & $1288.2(18)$ & $78.5(5)$ \\
N005 & $6405.6(17)$ & $4039.0(6)$ & $4117.1(16)$ & $45.6(4)$ \\
O006 & $5846(2)$ & $2800.1(6)$ & $3546.0(17)$ & $79.1(5)$ \\
N007 & $8263(2)$ & $4211.5(7)$ & $7028.9(18)$ & $60.7(4)$ \\
C008 & $5504.2(18)$ & $3675.0(6)$ & $477.1(18)$ & $38.1(4)$ \\
C009 & $5774.7(19)$ & $3415.5(6)$ & $-711.6(18)$ & $40.3(4)$ \\
C00A & $5530.6(19)$ & $3481.5(6)$ & $1756.8(18)$ & $38.6(4)$ \\
C00B & $3679(2)$ & $3907.4(6)$ & $2587(2)$ & $43.8(4)$ \\
C00C & $5213.5(19)$ & $3946.5(6)$ & $2576.5(18)$ & $39.4(4)$ \\
C00D & $5684(2)$ & $2918.8(7)$ & $2314(2)$ & $46.7(4)$ \\
C00E & $7802(2)$ & $4216.3(7)$ & $4437(2)$ & $45.2(4)$
\end{tabular}




\begin{tabular}{lcccc} 
C00F & $8851(2)$ & $4308.0(7)$ & $6093(2)$ & $46.3(4)$ \\
C00G & $5126(2)$ & $4443.7(7)$ & $1559(2)$ & $43.8(4)$ \\
C00H & $7019(2)$ & $3075.5(7)$ & $-339(2)$ & $51.0(5)$ \\
C00I & $10306(2)$ & $4500.8(8)$ & $6572(2)$ & $57.9(5)$ \\
C00J & $4809(2)$ & $3528.6(8)$ & $-2230(2)$ & $55.6(5)$ \\
C00K & $2605(2)$ & $3514.2(8)$ & $1772(2)$ & $56.8(5)$ \\
C00L & $3329(3)$ & $4286.8(8)$ & $3414(2)$ & $60.4(5)$ \\
C00M & $7287(3)$ & $2854.9(9)$ & $-1463(3)$ & $67.8(6)$ \\
C00N & $5074(3)$ & $3291.3(9)$ & $-3351(2)$ & $70.6(6)$ \\
C00O & $11206(3)$ & $4608.9(9)$ & $8098(3)$ & $69.8(6)$ \\
C00P & $6313(3)$ & $2960.4(9)$ & $-2961(3)$ & $72.0(7)$ \\
C00Q & $10625(3)$ & $4508.3(9)$ & $9074(3)$ & $73.2(6)$ \\
C00R & $1943(3)$ & $4259.3(10)$ & $3433(3)$ & $79.3(7)$ \\
C00S & $9162(3)$ & $4310.4(10)$ & $8500(3)$ & $75.6(6)$ \\
C00T & $1224(3)$ & $3492.3(9)$ & $1799(3)$ & $78.6(7)$ \\
C00U & $905(3)$ & $3862.0(11)$ & $2639(3)$ & $86.3(8)$ \\
C00V & $5630(4)$ & $1981.4(8)$ & $1724(4)$ & $118.2(12)$ \\
\hline
\end{tabular}

Table S3 Anisotropic Displacement Parameters $\left(\AA^{2} \times 10^{3}\right)$ for 31. The Anisotropic displacement factor exponent takes the form: $-2 \pi^{2}\left[h^{2} a^{* 2} U_{11}+2 h k a * b * U_{12}+\ldots\right]$.

\begin{tabular}{ccccccc}
\hline Atom & $\mathrm{U}_{11}$ & $\mathrm{U}_{22}$ & $\mathrm{U}_{33}$ & $\mathrm{U}_{23}$ & $\mathrm{U}_{13}$ & $\mathrm{U}_{12}$ \\
\hline $\mathrm{O} 001$ & $94.5(10)$ & $32.3(6)$ & $70.8(9)$ & $5.1(6)$ & $57.5(8)$ & $5.3(6)$ \\
$\mathrm{O} 002$ & $65.0(9)$ & $74.0(9)$ & $59.6(8)$ & $-2.9(7)$ & $41.2(7)$ & $-10.7(7)$ \\
$\mathrm{N} 003$ & $59.1(9)$ & $35.9(7)$ & $44.0(8)$ & $6.7(6)$ & $31.7(7)$ & $2.7(6)$ \\
O004 & $144.7(15)$ & $33.2(7)$ & $92.0(11)$ & $-3.9(7)$ & $84.1(11)$ & $-7.3(8)$ \\
$\mathrm{N} 005$ & $55.2(9)$ & $45.5(8)$ & $45.5(8)$ & $-1.3(6)$ & $31.5(7)$ & $-7.0(7)$ \\
O006 & $136.6(15)$ & $50.6(8)$ & $65.3(9)$ & $16.4(7)$ & $60(1)$ & $11.7(8)$ \\
N007 & $73.7(12)$ & $60.3(10)$ & $51.2(10)$ & $-1.5(8)$ & $32.1(9)$ & $-11.3(8)$ \\
C008 & $38.0(9)$ & $35.6(8)$ & $43.0(9)$ & $0.0(7)$ & $21.0(7)$ & $-2.2(7)$ \\
C009 & $48.1(10)$ & $37.4(8)$ & $40.9(9)$ & $-1.7(7)$ & $25.6(8)$ & $-6.8(7)$ \\
C00A & $43.1(9)$ & $35.2(8)$ & $43.8(9)$ & $1.5(7)$ & $25.5(8)$ & $0.4(7)$ \\
C00B & $52.8(11)$ & $36.6(9)$ & $53.7(10)$ & $12.1(8)$ & $34.7(9)$ & $8.5(7)$ \\
C00C & $48.5(10)$ & $33.2(8)$ & $44.1(9)$ & $2.6(7)$ & $28.0(8)$ & $0.1(7)$ \\
C00D & $57.2(11)$ & $38.1(9)$ & $56.9(11)$ & $4.7(8)$ & $36.8(9)$ & $5.1(8)$ \\
C00E & $53.5(11)$ & $36.2(9)$ & $54.2(11)$ & $-1.6(8)$ & $32.4(9)$ & $-0.9(8)$ \\
C00F & $54.6(11)$ & $34.6(8)$ & $51.6(10)$ & $2.2(7)$ & $26.7(9)$ & $1.6(7)$ \\
C00G & $53.3(11)$ & $35.6(9)$ & $53.5(10)$ & $3.6(7)$ & $34.2(9)$ & $-0.2(7)$ \\
C00H & $54.7(12)$ & $54.0(11)$ & $51.0(11)$ & $-2.1(8)$ & $30.5(9)$ & $2.2(9)$ \\
C00I & $54.7(12)$ & $53.6(11)$ & $64.7(13)$ & $5.4(9)$ & $27.4(10)$ & $3.5(9)$
\end{tabular}




\begin{tabular}{lcccccc} 
C00J & $66.9(13)$ & $53.4(11)$ & $45.4(10)$ & $5.3(8)$ & $25.5(10)$ & $2.1(9)$ \\
C00K & $53.6(12)$ & $46.2(10)$ & $79.1(14)$ & $4.8(9)$ & $38.3(11)$ & $2.7(9)$ \\
C00L & $77.7(14)$ & $48.3(11)$ & $75.1(14)$ & $5.7(10)$ & $52.4(12)$ & $11.3(10)$ \\
C00M & $86.1(16)$ & $61.4(13)$ & $77.7(15)$ & $-4.2(11)$ & $56.6(14)$ & $7.6(11)$ \\
C00N & $108.7(19)$ & $61.2(13)$ & $41.4(11)$ & $-0.8(9)$ & $35.0(12)$ & $-11.3(13)$ \\
C00O & $53.7(13)$ & $64.0(13)$ & $72.6(15)$ & $5.9(11)$ & $13.8(11)$ & $2.4(10)$ \\
C00P & $124(2)$ & $52.1(12)$ & $67.3(14)$ & $-10.7(11)$ & $67.3(15)$ & $-7.4(13)$ \\
C00Q & $76.8(16)$ & $66.8(14)$ & $53.4(12)$ & $2.3(11)$ & $11.9(12)$ & $0.9(12)$ \\
C00R & $99.5(19)$ & $67.5(14)$ & $110(2)$ & $23.1(14)$ & $81.9(17)$ & $34.5(14)$ \\
C00S & $89.5(18)$ & $83.9(16)$ & $52.9(13)$ & $-0.1(11)$ & $32.9(13)$ & $-11.8(13)$ \\
C00T & $56.7(14)$ & $63.9(14)$ & $128(2)$ & $18.5(14)$ & $53.9(15)$ & $3.8(11)$ \\
C00U & $76.9(17)$ & $75.3(16)$ & $141(2)$ & $38.6(16)$ & $79.1(18)$ & $24.4(14)$ \\
C00V & $213(4)$ & $29.7(11)$ & $173(3)$ & $2.7(15)$ & $141(3)$ & $0.9(16)$ \\
\hline
\end{tabular}

Table S4 Bond Lengths for 31 .

\begin{tabular}{llclll}
\hline Atom & Atom & Length/A & Atom & Atom & Length/A \\
\hline O001 & C00G & $1.2172(19)$ & C00B & C00C & $1.530(2)$ \\
O002 & C00E & $1.227(2)$ & C00B & C00K & $1.379(3)$ \\
N003 & C008 & $1.400(2)$ & C00B & C00L & $1.389(2)$ \\
N003 & C00G & $1.352(2)$ & C00C & C00G & $1.558(2)$ \\
O004 & C00D & $1.322(2)$ & C00E & C00F & $1.498(3)$ \\
O004 & C00V & $1.440(2)$ & C00F & C00I & $1.373(3)$ \\
N005 & C00C & $1.449(2)$ & C00H & C00M & $1.378(2)$ \\
N005 & C00E & $1.339(2)$ & C00I & C00O & $1.380(3)$ \\
O006 & C00D & $1.197(2)$ & C00J & C00N & $1.389(3)$ \\
N007 & C00F & $1.333(2)$ & C00K & C00T & $1.384(3)$ \\
N007 & C00S & $1.332(3)$ & C00L & C00R & $1.385(3)$ \\
C008 & C009 & $1.475(2)$ & C00M & C00P & $1.369(3)$ \\
C008 & C00A & $1.349(2)$ & C00N & C00P & $1.365(3)$ \\
C009 & C00H & $1.385(2)$ & C00O & C00Q & $1.366(3)$ \\
C009 & C00J & $1.387(2)$ & C00Q & C00S & $1.373(3)$ \\
C00A & C00C & $1.516(2)$ & C00R & C00U & $1.364(4)$ \\
C00A & C00D & $1.463(2)$ & C00T & C00U & $1.366(3)$ \\
\hline
\end{tabular}

Table S5 Bond Angles for 31.

\begin{tabular}{lcccc}
\hline Atom Atom Atom & Angle $^{\circ}$ & Atom & Atom Atom & Angle $^{\circ}$ \\
\hline C00G N003 C008 & $112.03(13)$ & O006 C00D C00A & $123.85(16)$ \\
C00D O004 C00V & $117.15(17)$ & O002 C00E N005 & $122.85(17)$ \\
C00E N005 C00C & $122.69(14)$ & O002 C00E C00F & $122.58(16)$
\end{tabular}




\begin{tabular}{llll} 
C00S N007 C00F & $116.88(19)$ & N005 C00E C00F & $114.55(15)$ \\
N003 C008 C009 & $116.98(13)$ & N007 C00F C00E & $116.08(16)$ \\
C00A C008 N003 & $110.22(13)$ & N007 C00F C00I & $123.45(18)$ \\
C00A C008 C009 & $132.78(15)$ & C00I C00F C00E & $120.42(16)$ \\
C00H C009 C008 & $121.14(15)$ & O001 C00G N003 & $126.62(15)$ \\
C00H C009 C00J & $118.90(16)$ & O001 C00G C00C & $126.66(15)$ \\
C00J C009 C008 & $119.90(16)$ & N003 C00G C00C & $106.65(13)$ \\
C008 C00A C00C & $109.32(13)$ & C00M C00H C009 & $120.28(18)$ \\
C008 C00A C00D & $129.83(15)$ & C00F C00I C00O & $118.5(2)$ \\
C00D C00A C00C & $120.63(13)$ & C009 C00J C00N & $120.03(19)$ \\
C00K C00B C00C & $122.29(15)$ & C00B C00K C00T & $120.8(2)$ \\
C00K C00B C00L & $118.34(17)$ & C00R C00L C00B & $120.2(2)$ \\
C00L C00B C00C & $119.35(16)$ & C00P C00M C00H & $120.5(2)$ \\
N005 C00C C00A & $114.55(14)$ & C00P C00N C00J & $120.2(2)$ \\
N005 C00C C00B & $108.71(13)$ & C00Q C00O C00I & $118.7(2)$ \\
N005 C00C C00G & $109.66(13)$ & C00N C00P C00M & $120.06(19)$ \\
C00A C00C C00B & $114.60(13)$ & C00O C00Q C00S & $118.9(2)$ \\
C00A C00C C00G & $101.24(12)$ & C00U C00R C00L & $120.6(2)$ \\
C00B C00C C00G & $107.58(13)$ & N007 C00S C00Q & $123.5(2)$ \\
O004 C00D C00A & $113.28(15)$ & C00U C00T C00K & $120.2(2)$ \\
O006 C00D O004 & $122.76(16)$ & C00R C00U C00T & $119.8(2)$ \\
\hline
\end{tabular}

Table S6 Hydrogen Atom Coordinates $\left(\AA \times 10^{4}\right)$ and Isotropic Displacement Parameters $\left(\AA^{2} \times 10^{3}\right)$ for 31 .

\begin{tabular}{lcccc}
\hline Atom & $\mathrm{x}$ & $\mathrm{y}$ & $\mathrm{z}$ & $\mathrm{U}(\mathrm{eq})$ \\
\hline H003 & 5057 & 4429 & -445 & 52 \\
H005 & 6208 & 3977 & 4856 & 55 \\
H00H & 7677 & 2996 & 675 & 61 \\
H00I & 10678 & 4557 & 5883 & 70 \\
H00J & 3983 & 3763 & -2499 & 67 \\
H00K & 2812 & 3261 & 1196 & 68 \\
H00L & 4027 & 4560 & 3957 & 73 \\
H00M & 8136 & 2633 & -1203 & 81 \\
H00N & 4406 & 3358 & -4370 & 85 \\
H00O & 12188 & 4747 & 8455 & 84 \\
H00P & 6495 & 2806 & -3714 & 86 \\
H00Q & 11210 & 4573 & 10110 & 88 \\
H00R & 1718 & 4514 & 3992 & 95 \\
H00S & 8778 & 4242 & 9174 & 91 \\
H00T & 510 & 3225 & 1244 & 94 \\
H00U & -17 & 3843 & 2669 & 104
\end{tabular}


H00A

\section{X-Ray crystal structure and data for compound 4a}

\section{(CCDC-1062309)}

X-Ray single-crystal diffraction data of crystals $\mathbf{4 a}$ was collected by using $\mathrm{Cu} \mathrm{K \alpha}$ radiation. The crystal was kept at $293.15 \mathrm{~K}$ during data collection. Using Olex2, the structure was solved with the Superflip structure solution program using Charge Flipping and refined with the ShelXL refinement package using Least Squares minimisation.

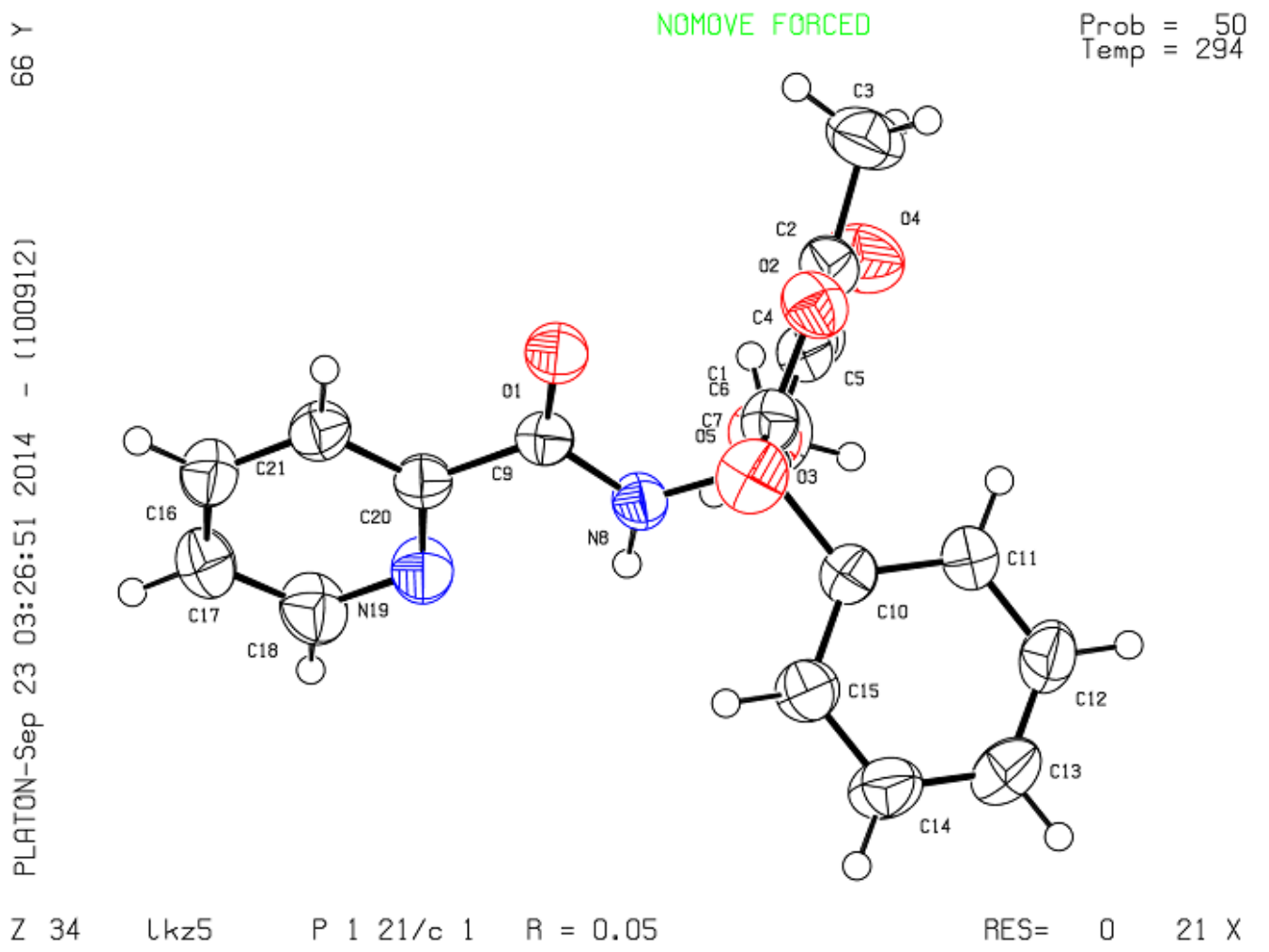

Figure S2 Crystal structure of 4 a (The ellipsoid contour probability level is $\mathbf{5 0 \%}$ )

Table S7 Crystal data and structure refinement for $4 a$.

\begin{tabular}{lc}
\hline Identification code & $\mathbf{4 a}$ \\
\hline Empirical formula & $\mathrm{C}_{19} \mathrm{H}_{16} \mathrm{~N}_{2} \mathrm{O}_{5}$
\end{tabular}


Formula weight

Temperature/K

Crystal system

Space group

$\mathrm{a} / \AA$

$\mathrm{b} / \AA$

$\mathrm{c} / \AA$

$\alpha /{ }^{\circ}$

$\beta /^{\circ}$

$\gamma /{ }^{\circ}$

Volume $/ \AA^{3}$

Z

$\rho_{\text {calc }} \mathrm{g} / \mathrm{cm}^{3}$

$\mu / \mathrm{mm}^{-1}$

$\mathrm{F}(000)$

Crystal size $/ \mathrm{mm}^{3}$

Radiation

$2 \Theta$ range for data collection/ ${ }^{\circ}$

Index ranges

Reflections collected

Independent reflections

Data/restraints/parameters

Goodness-of-fit on $\mathrm{F}^{2}$

Final $\mathrm{R}$ indexes $[\mathrm{I}>=2 \sigma(\mathrm{I})]$

Final $\mathrm{R}$ indexes [all data]

Largest diff. peak/hole / e $\AA^{-3}$
352.34

293.50(18)

monoclinic

$\mathrm{P} 2{ }_{1} / \mathrm{c}$

8.2709(3)

$11.1075(4)$

$18.7990(7)$

90

94.903(3)

90

1720.73(10)

4

1.360

0.833

736.0

$0.3 \times 0.3 \times 0.3$

$\mathrm{CuK} \alpha(\lambda=1.54184)$

9.256 to 134.15

$-9 \leq \mathrm{h} \leq 6,-13 \leq \mathrm{k} \leq 12,-22 \leq 1 \leq 21$

16699

$3072\left[\mathrm{R}_{\mathrm{int}}=0.0498, \mathrm{R}_{\text {sigma }}=0.0206\right]$

$3072 / 0 / 237$

1.067

$\mathrm{R}_{1}=0.0510, \mathrm{wR}_{2}=0.1360$

$\mathrm{R}_{1}=0.0536, \mathrm{wR}_{2}=0.1396$

$0.21 /-0.34$

Table S8 Fractional Atomic Coordinates $\left(\times 10^{4}\right)$ and Equivalent Isotropic Displacement Parameters $\left(\AA^{2} \times 10^{3}\right)$ for $4 a$. $U_{\text {eq }}$ is defined as $1 / 3$ of of the trace of the orthogonalised $\mathrm{U}_{\mathrm{IJ}}$ tensor.

\begin{tabular}{lcccc}
\hline Atom & $x$ & $y$ & $z$ & $\mathrm{U}(\mathrm{eq})$ \\
\hline O1 & $3139.5(15)$ & $1199.8(10)$ & $2233.7(6)$ & $53.5(3)$ \\
O3 & $5921.5(13)$ & $1835.9(12)$ & $1405.9(7)$ & $58.4(3)$ \\
O2 & $4661.3(13)$ & $3457(1)$ & $1806.3(6)$ & $53.5(3)$ \\
N8 & $2371.0(14)$ & $1168.4(11)$ & $1054.6(6)$ & $41.7(3)$ \\
C7 & $3124.8(17)$ & $2314.0(12)$ & $915.3(7)$ & $39.4(3)$ \\
O5 & $-126.7(13)$ & $2875.4(11)$ & $477.3(7)$ & $56.4(3)$ \\
N19 & $645.5(17)$ & $-840.6(13)$ & $1193.9(7)$ & $52.0(4)$
\end{tabular}




\begin{tabular}{lcccc} 
C20 & $1489.2(16)$ & $-439.2(13)$ & $1784.7(7)$ & $39.3(3)$ \\
C9 & $2400.0(17)$ & $713.7(13)$ & $1718.6(7)$ & $40.1(3)$ \\
C10 & $3565.3(17)$ & $2381.2(13)$ & $142.7(8)$ & $40.7(3)$ \\
C21 & $1574.4(19)$ & $-1036.6(14)$ & $2427.8(8)$ & $47.2(4)$ \\
C4 & $2217.9(18)$ & $3392.5(13)$ & $1163.6(8)$ & $42.2(3)$ \\
O4 & $-82.5(17)$ & $4676.4(12)$ & $1009.7(8)$ & $70.1(4)$ \\
C5 & $570.6(18)$ & $3746.0(14)$ & $890.4(8)$ & $45.8(4)$ \\
C1 & $4731.6(18)$ & $2429.7(14)$ & $1400.7(8)$ & $44.8(4)$ \\
C11 & $3664(2)$ & $3477.3(15)$ & $-200.3(9)$ & $51.0(4)$ \\
C16 & $734(2)$ & $-2103.7(15)$ & $2471.9(9)$ & $53.6(4)$ \\
C2 & $3137(2)$ & $3971.4(14)$ & $1670.6(9)$ & $48.6(4)$ \\
C15 & $3933(2)$ & $1344.1(15)$ & $-219.7(9)$ & $54.6(4)$ \\
C17 & $-160(2)$ & $-2523.6(15)$ & $1875.3(10)$ & $55.7(4)$ \\
C18 & $-166(2)$ & $-1870.6(17)$ & $1253.2(10)$ & $59.9(5)$ \\
C13 & $4507(2)$ & $2502.6(18)$ & $-1242(1)$ & $61.1(5)$ \\
C12 & $4127(2)$ & $3530.2(17)$ & $-891.7(9)$ & $60.1(5)$ \\
C14 & $4407(3)$ & $1406.4(18)$ & $-906.4(10)$ & $63.5(5)$ \\
C3 & $2855(3)$ & $5018.2(17)$ & $2127.6(12)$ & $70.9(5)$ \\
C6 & $-1752(2)$ & $3117(2)$ & $165.7(13)$ & $74.5(6)$ \\
\hline
\end{tabular}

Table S9 Anisotropic Displacement Parameters $\left(\AA^{2} \times 10^{3}\right)$ for $4 a$. The Anisotropic displacement factor exponent takes the form: $-2 \pi^{2}\left[h^{2} a^{* 2} U_{11}+2 h k a * b * U_{12}+\ldots\right]$.

\begin{tabular}{lcccccc}
\hline Atom & $\mathrm{U}_{11}$ & $\mathrm{U}_{22}$ & $\mathrm{U}_{33}$ & $\mathrm{U}_{23}$ & $\mathrm{U}_{13}$ & $\mathrm{U}_{12}$ \\
\hline $\mathrm{O} 1$ & $65.8(7)$ & $52.9(6)$ & $40.7(6)$ & $-4.9(5)$ & $-2.5(5)$ & $-13.3(5)$ \\
$\mathrm{O} 3$ & $44.4(6)$ & $68.7(8)$ & $60.7(7)$ & $7.2(6)$ & $-4.3(5)$ & $7.4(5)$ \\
$\mathrm{O} 2$ & $49.7(6)$ & $52.3(6)$ & $56.4(7)$ & $-5.2(5)$ & $-6.7(5)$ & $-9.0(5)$ \\
$\mathrm{N} 8$ & $48.4(7)$ & $38.9(6)$ & $36.8(6)$ & $0.3(5)$ & $-2.5(5)$ & $-4.5(5)$ \\
$\mathrm{C} 7$ & $39.6(7)$ & $38.2(7)$ & $39.8(7)$ & $1.6(5)$ & $-0.7(6)$ & $-0.3(5)$ \\
O5 & $42.3(6)$ & $60.7(7)$ & $64.3(7)$ & $-2.7(5)$ & $-6.9(5)$ & $2.5(5)$ \\
$\mathrm{N} 19$ & $55.1(7)$ & $53.8(8)$ & $45.1(7)$ & $1.7(6)$ & $-7.1(6)$ & $-10.1(6)$ \\
$\mathrm{C} 20$ & $37.5(7)$ & $40.6(7)$ & $39.9(7)$ & $-1.3(6)$ & $3.1(5)$ & $2.4(5)$ \\
$\mathrm{C} 9$ & $41.1(7)$ & $40.2(7)$ & $39.0(7)$ & $-2.4(5)$ & $2.9(6)$ & $2.1(6)$ \\
$\mathrm{C} 10$ & $37.0(7)$ & $44.5(8)$ & $39.8(8)$ & $4.3(6)$ & $-0.5(5)$ & $3.9(6)$ \\
$\mathrm{C} 21$ & $50.9(8)$ & $50.3(8)$ & $39.6(8)$ & $0.4(6)$ & $-0.4(6)$ & $-1.8(7)$ \\
$\mathrm{C} 4$ & $44.6(7)$ & $39.3(7)$ & $42.6(8)$ & $0.3(6)$ & $4.4(6)$ & $-1.4(6)$ \\
$\mathrm{O} 4$ & $69.4(8)$ & $62.8(8)$ & $77.4(9)$ & $-7.1(6)$ & $2.2(7)$ & $23.6(6)$ \\
$\mathrm{C} 5$ & $45.8(8)$ & $47.4(8)$ & $44.9(8)$ & $4.1(6)$ & $8.8(6)$ & $4.3(6)$ \\
C1 & $42.5(8)$ & $47.6(8)$ & $43.5(8)$ & $5.4(6)$ & $-1.6(6)$ & $-2.9(6)$ \\
C11 & $59.1(9)$ & $43.1(8)$ & $51.4(9)$ & $2.8(7)$ & $7.3(7)$ & $-4.0(7)$
\end{tabular}




\begin{tabular}{lcccccc} 
C16 & $59.2(9)$ & $50.3(9)$ & $51.4(9)$ & $8.9(7)$ & $6.3(7)$ & $-1.7(7)$ \\
C2 & $52.5(8)$ & $42.4(8)$ & $50.8(9)$ & $-1.3(6)$ & $4.0(7)$ & $-4.7(6)$ \\
C15 & $67.8(10)$ & $47.4(9)$ & $48.9(9)$ & $6.9(7)$ & $7.0(7)$ & $17.8(7)$ \\
C17 & $52.8(9)$ & $47.5(9)$ & $66.6(11)$ & $2.8(7)$ & $4.9(8)$ & $-8.3(7)$ \\
C18 & $58.3(10)$ & $59.9(10)$ & $58.9(10)$ & $-2.7(8)$ & $-10.4(8)$ & $-16.4(8)$ \\
C13 & $64.9(11)$ & $77.3(12)$ & $41.8(9)$ & $7.1(8)$ & $7.9(7)$ & $5.8(9)$ \\
C12 & $72.0(11)$ & $57.4(10)$ & $51.5(9)$ & $14.7(8)$ & $8.7(8)$ & $-5.3(8)$ \\
C14 & $78.1(12)$ & $64.4(11)$ & $49.3(9)$ & $1.1(8)$ & $12.0(8)$ & $22.0(9)$ \\
C3 & $82.7(13)$ & $56.6(10)$ & $73.2(13)$ & $-22.2(9)$ & $5.4(10)$ & $-6.6(9)$ \\
C6 & $43.1(9)$ & $97.9(16)$ & $80.0(13)$ & $6.1(11)$ & $-8.6(9)$ & $2.0(9)$ \\
\hline
\end{tabular}

Table S10 Bond Lengths for $4 a$.

\begin{tabular}{|c|c|c|c|c|c|}
\hline Atom & Atom & Length/Å & Atom & Atom & Length/Å \\
\hline $\mathrm{O} 1$ & C9 & $1.2257(17)$ & $\mathrm{C} 20$ & $\mathrm{C} 21$ & $1.376(2)$ \\
\hline $\mathrm{O} 3$ & $\mathrm{C} 1$ & $1.1840(19)$ & $\mathrm{C} 10$ & $\mathrm{C} 11$ & $1.383(2)$ \\
\hline $\mathrm{O} 2$ & $\mathrm{C} 1$ & $1.3763(19)$ & $\mathrm{C} 10$ & C15 & $1.385(2)$ \\
\hline $\mathrm{O} 2$ & $\mathrm{C} 2$ & $1.388(2)$ & $\mathrm{C} 21$ & C16 & $1.380(2)$ \\
\hline N8 & $\mathrm{C} 7$ & $1.4507(18)$ & $\mathrm{C} 4$ & $\mathrm{C} 5$ & $1.468(2)$ \\
\hline N8 & C9 & $1.3448(18)$ & $\mathrm{C} 4$ & $\mathrm{C} 2$ & $1.333(2)$ \\
\hline $\mathrm{C} 7$ & $\mathrm{C} 10$ & $1.529(2)$ & O4 & $\mathrm{C} 5$ & $1.1962(19)$ \\
\hline $\mathrm{C} 7$ & $\mathrm{C} 4$ & $1.508(2)$ & $\mathrm{C} 11$ & $\mathrm{C} 12$ & $1.387(2)$ \\
\hline $\mathrm{C} 7$ & $\mathrm{C} 1$ & $1.5519(19)$ & C16 & $\mathrm{C} 17$ & $1.371(3)$ \\
\hline $\mathrm{O} 5$ & $\mathrm{C} 5$ & $1.340(2)$ & $\mathrm{C} 2$ & $\mathrm{C} 3$ & $1.476(2)$ \\
\hline $\mathrm{O} 5$ & C6 & $1.445(2)$ & C15 & C14 & $1.383(2)$ \\
\hline N19 & $\mathrm{C} 20$ & $1.3368(19)$ & $\mathrm{C} 17$ & $\mathrm{C} 18$ & $1.376(3)$ \\
\hline N19 & C18 & $1.336(2)$ & C13 & $\mathrm{C} 12$ & $1.368(3)$ \\
\hline $\mathrm{C} 20$ & C9 & $1.496(2)$ & $\mathrm{C} 13$ & $\mathrm{C} 14$ & $1.377(3)$ \\
\hline
\end{tabular}

Table S11 Bond Angles for 4a.

\begin{tabular}{|c|c|c|c|c|c|c|c|}
\hline Atom & Atom & Atom & Angle $/^{\circ}$ & Atom & Atom & Atom & Angle $/^{\circ}$ \\
\hline $\mathrm{C} 1$ & $\mathrm{O} 2$ & $\mathrm{C} 2$ & 108.68(11) & $\mathrm{C} 5$ & $\mathrm{C} 4$ & $\mathrm{C} 7$ & $125.12(13)$ \\
\hline C9 & N8 & $\mathrm{C} 7$ & $121.55(12)$ & $\mathrm{C} 2$ & $\mathrm{C} 4$ & $\mathrm{C} 7$ & $109.65(13)$ \\
\hline N8 & $\mathrm{C} 7$ & $\mathrm{C} 10$ & $110.81(11)$ & $\mathrm{C} 2$ & $\mathrm{C} 4$ & $\mathrm{C} 5$ & $125.23(14)$ \\
\hline N8 & $\mathrm{C} 7$ & $\mathrm{C} 4$ & $114.08(12)$ & O5 & C5 & $\mathrm{C} 4$ & $110.52(13)$ \\
\hline N8 & $\mathrm{C} 7$ & $\mathrm{C} 1$ & $109.03(11)$ & $\mathrm{O} 4$ & $\mathrm{C} 5$ & O5 & $123.61(15)$ \\
\hline $\mathrm{C} 10$ & $\mathrm{C} 7$ & $\mathrm{C} 1$ & $107.03(11)$ & $\mathrm{O} 4$ & $\mathrm{C} 5$ & $\mathrm{C} 4$ & $125.87(15)$ \\
\hline $\mathrm{C} 4$ & $\mathrm{C} 7$ & $\mathrm{C} 10$ & $114.86(11)$ & $\mathrm{O} 3$ & $\mathrm{C} 1$ & $\mathrm{O} 2$ & $122.13(14)$ \\
\hline $\mathrm{C} 4$ & $\mathrm{C} 7$ & $\mathrm{C} 1$ & $100.12(11)$ & $\mathrm{O} 3$ & $\mathrm{C} 1$ & $\mathrm{C} 7$ & $128.91(15)$ \\
\hline $\mathrm{C} 5$ & O5 & C6 & $116.13(15)$ & $\mathrm{O} 2$ & $\mathrm{C} 1$ & $\mathrm{C} 7$ & $108.65(12)$ \\
\hline
\end{tabular}




\begin{tabular}{cccccccc} 
C18 & N19 & C20 & $116.54(14)$ & C10 & C11 & C12 & $120.40(16)$ \\
N19 & C20 & C9 & $116.57(12)$ & C17 & C16 & C21 & $118.84(15)$ \\
N19 & C20 & C21 & $123.59(14)$ & O2 & C2 & C3 & $113.81(15)$ \\
C21 & C20 & C9 & $119.84(13)$ & C4 & C2 & O2 & $112.73(14)$ \\
O1 & C9 & N8 & $122.41(13)$ & C4 & C2 & C3 & $133.44(17)$ \\
O1 & C9 & C20 & $122.15(13)$ & C14 & C15 & C10 & $120.61(15)$ \\
N8 & C9 & C20 & $115.43(12)$ & C16 & C17 & C18 & $118.58(16)$ \\
C11 & C10 & C7 & $120.93(13)$ & N19 & C18 & C17 & $123.85(16)$ \\
C11 & C10 & C15 & $118.59(14)$ & C12 & C13 & C14 & $119.48(16)$ \\
C15 & C10 & C7 & $120.44(13)$ & C13 & C12 & C11 & $120.59(16)$ \\
C20 & C21 & C16 & $118.60(15)$ & C13 & C14 & C15 & $120.33(17)$ \\
\hline
\end{tabular}

Table S12 Torsion Angles for 4a.

\begin{tabular}{|c|c|c|c|c|c|c|c|c|c|}
\hline A & B & $\mathrm{C}$ & $\mathrm{D}$ & Angle $/^{\circ}$ & A & B & $\mathrm{C}$ & $\mathrm{D}$ & Angle $/^{\circ}$ \\
\hline N8 & $\mathrm{C} 7$ & $\mathrm{C} 10$ & C11 & $153.40(14)$ & $\mathrm{C} 21$ & $\mathrm{C} 20$ & C9 & $\mathrm{O} 1$ & $-5.3(2)$ \\
\hline N8 & $\mathrm{C} 7$ & $\mathrm{C} 10$ & $\mathrm{C} 15$ & $-28.98(18)$ & $\mathrm{C} 21$ & $\mathrm{C} 20$ & $\mathrm{C} 9$ & N8 & $173.74(13)$ \\
\hline N8 & $\mathrm{C} 7$ & $\mathrm{C} 4$ & C5 & $-63.16(18)$ & $\mathrm{C} 21$ & $\mathrm{C} 16$ & $\mathrm{C} 17$ & $\mathrm{C} 18$ & $0.8(3)$ \\
\hline N8 & $\mathrm{C} 7$ & $\mathrm{C} 4$ & $\mathrm{C} 2$ & $116.56(14)$ & $\mathrm{C} 4$ & $\mathrm{C} 7$ & $\mathrm{C} 10$ & $\mathrm{C} 11$ & 22.32(19) \\
\hline N8 & $\mathrm{C} 7$ & $\mathrm{C} 1$ & $\mathrm{O} 3$ & $63.8(2)$ & $\mathrm{C} 4$ & $\mathrm{C} 7$ & $\mathrm{C} 10$ & $\mathrm{C} 15$ & $-160.07(14)$ \\
\hline N8 & $\mathrm{C} 7$ & $\mathrm{C} 1$ & $\mathrm{O} 2$ & $-122.69(13)$ & $\mathrm{C} 4$ & $\mathrm{C} 7$ & $\mathrm{C} 1$ & $\mathrm{O} 3$ & $-176.24(16)$ \\
\hline $\mathrm{C} 7$ & N8 & C9 & $\mathrm{O} 1$ & $-4.4(2)$ & $\mathrm{C} 4$ & $\mathrm{C} 7$ & $\mathrm{C} 1$ & $\mathrm{O} 2$ & $-2.70(14)$ \\
\hline $\mathrm{C} 7$ & N8 & C9 & $\mathrm{C} 20$ & $176.56(12)$ & $\mathrm{C} 5$ & $\mathrm{C} 4$ & $\mathrm{C} 2$ & $\mathrm{O} 2$ & $-178.05(13)$ \\
\hline $\mathrm{C} 7$ & $\mathrm{C} 10$ & C11 & $\mathrm{C} 12$ & $177.44(15)$ & $\mathrm{C} 5$ & $\mathrm{C} 4$ & $\mathrm{C} 2$ & $\mathrm{C} 3$ & $3.9(3)$ \\
\hline $\mathrm{C} 7$ & $\mathrm{C} 10$ & $\mathrm{C} 15$ & $\mathrm{C} 14$ & $-176.89(15)$ & $\mathrm{C} 1$ & $\mathrm{O} 2$ & $\mathrm{C} 2$ & $\mathrm{C} 4$ & $-4.11(18)$ \\
\hline $\mathrm{C} 7$ & $\mathrm{C} 4$ & $\mathrm{C} 5$ & O5 & $10.3(2)$ & $\mathrm{C} 1$ & $\mathrm{O} 2$ & $\mathrm{C} 2$ & $\mathrm{C} 3$ & $174.34(14)$ \\
\hline $\mathrm{C} 7$ & $\mathrm{C} 4$ & $\mathrm{C} 5$ & $\mathrm{O} 4$ & $-169.40(15)$ & $\mathrm{C} 1$ & $\mathrm{C} 7$ & $\mathrm{C} 10$ & $\mathrm{C} 11$ & $-87.84(16)$ \\
\hline $\mathrm{C} 7$ & $\mathrm{C} 4$ & $\mathrm{C} 2$ & $\mathrm{O} 2$ & $2.23(18)$ & $\mathrm{C} 1$ & $\mathrm{C} 7$ & $\mathrm{C} 10$ & $\mathrm{C} 15$ & $89.78(16)$ \\
\hline $\mathrm{C} 7$ & $\mathrm{C} 4$ & $\mathrm{C} 2$ & $\mathrm{C} 3$ & $-175.81(18)$ & $\mathrm{C} 1$ & $\mathrm{C} 7$ & $\mathrm{C} 4$ & $\mathrm{C} 5$ & $-179.42(14)$ \\
\hline N19 & $\mathrm{C} 20$ & C9 & $\mathrm{O} 1$ & $175.85(14)$ & $\mathrm{C} 1$ & $\mathrm{C} 7$ & $\mathrm{C} 4$ & $\mathrm{C} 2$ & $0.30(15)$ \\
\hline N19 & $\mathrm{C} 20$ & $\mathrm{C} 9$ & N8 & $-5.12(19)$ & C11 & $\mathrm{C} 10$ & $\mathrm{C} 15$ & $\mathrm{C} 14$ & $0.8(2)$ \\
\hline N19 & $\mathrm{C} 20$ & $\mathrm{C} 21$ & C16 & $-0.6(2)$ & C16 & $\mathrm{C} 17$ & $\mathrm{C} 18$ & N19 & $-0.3(3)$ \\
\hline $\mathrm{C} 20$ & N19 & $\mathrm{C} 18$ & $\mathrm{C} 17$ & $-0.6(3)$ & $\mathrm{C} 2$ & $\mathrm{O} 2$ & $\mathrm{C} 1$ & $\mathrm{O} 3$ & $178.19(15)$ \\
\hline $\mathrm{C} 20$ & $\mathrm{C} 21$ & $\mathrm{C} 16$ & $\mathrm{C} 17$ & $-0.4(2)$ & $\mathrm{C} 2$ & $\mathrm{O} 2$ & $\mathrm{C} 1$ & $\mathrm{C} 7$ & $4.12(16)$ \\
\hline C9 & N8 & $\mathrm{C} 7$ & $\mathrm{C} 10$ & 155.91(12) & $\mathrm{C} 2$ & $\mathrm{C} 4$ & C5 & O5 & $-169.33(15)$ \\
\hline C9 & N8 & $\mathrm{C} 7$ & $\mathrm{C} 4$ & $-72.60(16)$ & $\mathrm{C} 2$ & $\mathrm{C} 4$ & $\mathrm{C} 5$ & $\mathrm{O} 4$ & $10.9(3)$ \\
\hline C9 & N8 & $\mathrm{C} 7$ & $\mathrm{C} 1$ & $38.36(18)$ & $\mathrm{C} 15$ & $\mathrm{C} 10$ & $\mathrm{C} 11$ & $\mathrm{C} 12$ & $-0.2(2)$ \\
\hline C9 & $\mathrm{C} 20$ & $\mathrm{C} 21$ & $\mathrm{C} 16$ & $-179.41(14)$ & $\mathrm{C} 18$ & N19 & $\mathrm{C} 20$ & $\mathrm{C} 9$ & $179.94(14)$ \\
\hline $\mathrm{C} 10$ & $\mathrm{C} 7$ & $\mathrm{C} 4$ & $\mathrm{C} 5$ & $66.34(18)$ & C18 & N19 & C20 & $\mathrm{C} 21$ & $1.1(2)$ \\
\hline $\mathrm{C} 10$ & $\mathrm{C} 7$ & $\mathrm{C} 4$ & $\mathrm{C} 2$ & $-113.94(14)$ & $\mathrm{C} 12$ & $\mathrm{C} 13$ & C14 & $\mathrm{C} 15$ & $-0.2(3)$ \\
\hline
\end{tabular}




$\begin{array}{cccccccccc}\mathrm{C} 10 & \mathrm{C} 7 & \mathrm{C} 1 & \mathrm{O} 3 & -56.2(2) & \mathrm{C} 14 & \mathrm{C} 13 & \mathrm{C} 12 & \mathrm{C} 11 & 0.8(3) \\ \mathrm{C} 10 & \mathrm{C} 7 & \mathrm{C} 1 & \mathrm{O} 2 & 117.39(13) & \mathrm{C} 6 & \mathrm{O} 5 & \mathrm{C} 5 & \mathrm{C} 4 & -179.25(14) \\ \mathrm{C} 10 & \mathrm{C} 11 & \mathrm{C} 12 & \mathrm{C} 13 & -0.6(3) & \mathrm{C} 6 & \mathrm{O} 5 & \mathrm{C} 5 & \mathrm{O} 4 & 0.5(2) \\ \mathrm{C} 10 & \mathrm{C} 15 & \mathrm{C} 14 & \mathrm{C} 13 & -0.6(3) & & & & & \end{array}$

Table S13 Hydrogen Atom Coordinates $\left(\AA \times 10^{4}\right)$ and Isotropic Displacement Parameters $\left(\AA^{2} \times 10^{3}\right)$ for 4 a.

\begin{tabular}{ccccc}
\hline Atom & $x$ & $y$ & $z$ & $\mathrm{U}(\mathrm{eq})$ \\
\hline H8 & 1895 & 771 & 704 & 50 \\
H21 & 2185 & -728 & 2825 & 57 \\
H11 & 3418 & 4183 & 34 & 61 \\
H16 & 774 & -2530 & 2899 & 64 \\
H15 & 3860 & 599 & 1 & 66 \\
H17 & -750 & -3235 & 1891 & 67 \\
H18 & -771 & -2163 & 850 & 72 \\
H13 & 4830 & 2544 & -1703 & 73 \\
H12 & 4180 & 4271 & -1119 & 72 \\
H14 & 4660 & 705 & -1143 & 76 \\
H3A & 2658 & 4745 & 2597 & 106 \\
H3B & 1930 & 5461 & 1926 & 106 \\
H3C & 3793 & 5530 & 2158 & 106 \\
H6A & -2248 & 2379 & -5 & 112 \\
H6B & -1707 & 3669 & -225 & 112 \\
H6C & -2380 & 3463 & 520 & 112 \\
\hline
\end{tabular}

\section{X-Ray crystal structure and data for compound 5a (CCDC-1404091)}

X-Ray single-crystal diffraction data of crystals 5a was collected by using $\mathrm{Cu} \mathrm{Ka}$ radiation. The crystal was kept at $293.15 \mathrm{~K}$ during data collection. Using Olex2, the structure was solved with the Superflip structure solution program using Charge Flipping and refined with the ShelXL refinement package using Least Squares minimisation. 


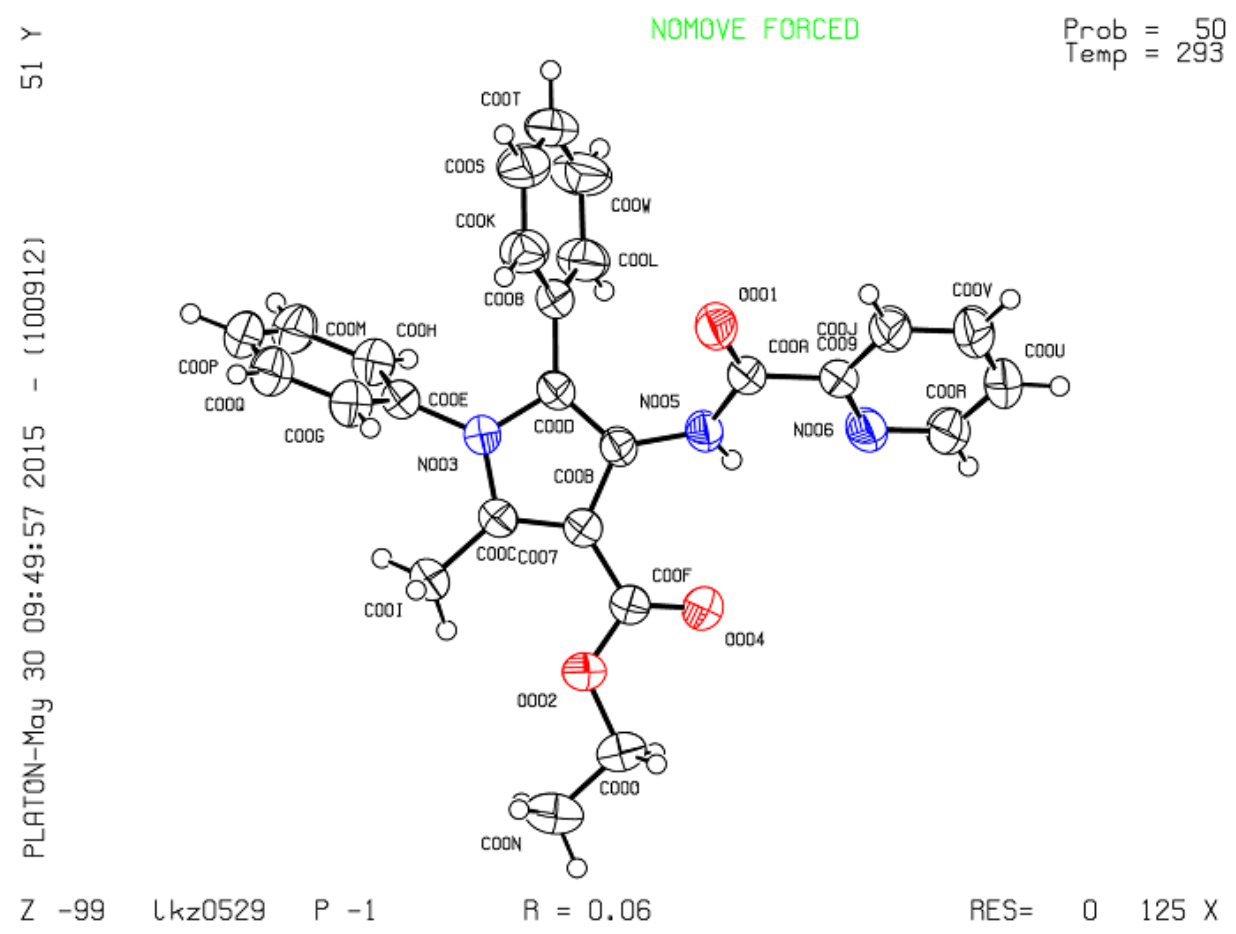

Figure S3 Crystal structure of 5a (The ellipsoid contour probability level is 50\%)

Table S14 Crystal data and structure refinement for 5a.

\begin{tabular}{ll}
\hline Identification code & $\mathbf{5 a}$ \\
\hline Empirical formula & $\mathrm{C}_{26} \mathrm{H}_{23} \mathrm{~N}_{3} \mathrm{O}_{3}$ \\
Formula weight & 425.47 \\
Temperature/K & 293 \\
Crystal system & triclinic \\
Space group & $\mathrm{P}-1$ \\
$\mathrm{a} / \AA$ & $10.5769(4)$ \\
$\mathrm{b} / \AA$ & $10.9393(4)$ \\
$\mathrm{c} / \AA$ & $11.0491(4)$ \\
$\alpha /{ }^{\circ}$ & $73.609(3)$ \\
$\beta /{ }^{\circ}$ & $78.749(3)$ \\
$\gamma /{ }^{\circ}$ & $65.477(4)$ \\
Volume $/ \AA^{3}$ & $1111.37(8)$ \\
$\mathrm{Z}$ & 2 \\
$\rho_{\text {calcg }} / \mathrm{cm}^{3}$ & 1.271 \\
$\mu / \mathrm{mm}^{-1}$ & 0.681 \\
$\mathrm{~F}(000)$ & 448.0 \\
$\mathrm{Crystal} \mathrm{size} / \mathrm{mm}^{3}$ & $0.3 \times 0.3 \times 0.3$ \\
Radiation & $\mathrm{CuK} \alpha(\lambda=1.54184)$
\end{tabular}


$2 \Theta$ range for data collection/ ${ }^{\circ}$

Index ranges

Reflections collected

Independent reflections

Data/restraints/parameters

Goodness-of-fit on $\mathrm{F}^{2}$

Final R indexes $[\mathrm{I}>=2 \sigma(\mathrm{I})]$

Final $\mathrm{R}$ indexes [all data]

Largest diff. peak/hole / e $\AA^{-3}$
9.228 to 134.116

$-12 \leq \mathrm{h} \leq 12,-13 \leq \mathrm{k} \leq 13,-13 \leq 1 \leq 13$

19394

$3957\left[\mathrm{R}_{\mathrm{int}}=0.0324, \mathrm{R}_{\text {sigma }}=0.0164\right]$

$3957 / 0 / 291$

1.048

$\mathrm{R}_{1}=0.0562, \mathrm{wR}_{2}=0.1439$

$\mathrm{R}_{1}=0.0586, \mathrm{wR}_{2}=0.1471$

$0.24 /-0.36$

Table S15 Fractional Atomic Coordinates $\left(\times 10^{4}\right)$ and Equivalent Isotropic Displacement Parameters $\left(\AA^{2} \times 10^{3}\right)$ for $5 \mathrm{a} . \mathrm{U}_{\text {eq }}$ is defined as $1 / 3$ of of the trace of the orthogonalised $U_{I J}$ tensor.

\begin{tabular}{|c|c|c|c|c|}
\hline Atom & $x$ & $y$ & $z$ & $\mathrm{U}(\mathrm{eq})$ \\
\hline O001 & $3865.0(12)$ & $8816.9(13)$ & $3203.2(12)$ & $61.1(3)$ \\
\hline $\mathrm{O} 002$ & $1737.2(14)$ & $4281.9(12)$ & $3709.9(12)$ & $64.4(3)$ \\
\hline N003 & 2984.7(13) & $5668.9(12)$ & $6241.0(11)$ & $46.0(3)$ \\
\hline O004 & $1098.3(16)$ & $6453.9(13)$ & $2591.0(12)$ & $71.2(4)$ \\
\hline N005 & 1991.9(13) & $8243.0(12)$ & $3365.0(12)$ & $47.1(3)$ \\
\hline N006 & $1018.0(15)$ & $10217.8(14)$ & $1339.4(14)$ & $59.0(4)$ \\
\hline $\mathrm{C} 007$ & $2188.5(15)$ & $5833.1(15)$ & $4466.0(13)$ & $43.7(3)$ \\
\hline C008 & $3062.8(16)$ & $7984.5(15)$ & $5998.5(13)$ & $45.7(3)$ \\
\hline C009 & $2253.0(16)$ & $10041.1(14)$ & $1635.8(14)$ & $46.4(3)$ \\
\hline $\mathrm{COOA}$ & $2794.9(15)$ & $8975.5(15)$ & $2816.1(14)$ & $44.7(3)$ \\
\hline $\mathrm{COOB}$ & $2306.2(15)$ & 7109.6(14) & $4411.9(13)$ & $42.9(3)$ \\
\hline $\mathrm{C} 00 \mathrm{C}$ & $2615.2(16)$ & $4964.0(15)$ & $5618.3(14)$ & $46.0(3)$ \\
\hline C00D & $2781.9(15)$ & 6999.4(14) & $5514.4(13)$ & $44.0(3)$ \\
\hline $\mathrm{COOE}$ & $3357.0(16)$ & $5174.6(15)$ & $7523.2(14)$ & $47.3(3)$ \\
\hline $\mathrm{COOF}$ & $1626.4(16)$ & $5582.3(15)$ & $3494.4(14)$ & $46.6(3)$ \\
\hline $\mathrm{CO0G}$ & $4611.8(19)$ & 4100.1(17) & 7796.8(17) & $58.6(4)$ \\
\hline $\mathrm{COOH}$ & 2456.4(19) & $5767.7(18)$ & $8461.2(16)$ & $57.6(4)$ \\
\hline COOI & $2676(2)$ & $3537.9(17)$ & $6217.2(16)$ & $58.7(4)$ \\
\hline $\mathrm{COOJ}$ & $3023(2)$ & $10795(2)$ & $930.5(17)$ & $63.4(5)$ \\
\hline $\mathrm{COOK}$ & $4390.2(18)$ & 7696.2(19) & $6286.6(18)$ & $59.2(4)$ \\
\hline $\mathrm{COOL}$ & 2003.0(19) & 9193.7(18) & 6192(2) & $64.1(5)$ \\
\hline $\mathrm{COOM}$ & $2820(2)$ & $5267(2)$ & $9698.3(17)$ & $69.3(5)$ \\
\hline $\mathrm{COON}$ & $1020(2)$ & $2575(2)$ & $3517(2)$ & $70.2(5)$ \\
\hline $\mathrm{C} 00 \mathrm{O}$ & $1133(2)$ & $3917(2)$ & 2873.8(19) & $66.6(5)$ \\
\hline COOP & $4050(2)$ & $4181(2)$ & $9980.5(18)$ & $72.1(5)$ \\
\hline
\end{tabular}




\begin{tabular}{lcccc} 
C00Q & $4955(2)$ & $3595(2)$ & $9042.0(19)$ & $70.5(5)$ \\
C00R & $523(2)$ & $11186.6(19)$ & $309(2)$ & $71.2(5)$ \\
C00S & $4643(2)$ & $8620(2)$ & $6742(2)$ & $72.3(5)$ \\
C00T & $3576(2)$ & $9821(2)$ & $6938(2)$ & $76.7(6)$ \\
C00U & $1212(3)$ & $11986(2)$ & $-439(2)$ & $77.6(6)$ \\
C00V & $2486(3)$ & $11784(2)$ & $-126.2(19)$ & $82.0(6)$ \\
C00W & $2257(2)$ & $10107(2)$ & $6670(3)$ & $82.2(6)$ \\
\hline
\end{tabular}

Table S16 Anisotropic Displacement Parameters $\left(\AA^{2} \times 10^{3}\right)$ for 5a. The Anisotropic displacement factor exponent takes the form: $-2 \pi^{2}\left[h^{2} a^{* 2} U_{11}+2 h k a * b * U_{12}+\ldots\right]$.

\begin{tabular}{|c|c|c|c|c|c|c|}
\hline Atom & $\mathrm{U}_{11}$ & $\mathrm{U}_{22}$ & $\mathrm{U}_{33}$ & $\mathrm{U}_{23}$ & $\mathrm{U}_{13}$ & $\mathrm{U}_{12}$ \\
\hline O001 & $61.3(7)$ & $65.3(7)$ & $61.7(7)$ & $6.8(5)$ & $-22.6(5)$ & $-35.4(6)$ \\
\hline O002 & $93.2(9)$ & $51.5(6)$ & $63.0(7)$ & $-5.4(5)$ & $-29.9(6)$ & $-36.4(6)$ \\
\hline N003 & $59.3(7)$ & $42.7(6)$ & $39.2(6)$ & $-3.4(5)$ & $-9.3(5)$ & $-24.1(5)$ \\
\hline O004 & $102.2(10)$ & $59.0(7)$ & $63.2(7)$ & $4.0(6)$ & $-40.1(7)$ & $-38.3(7)$ \\
\hline N005 & $49.7(7)$ & $44.9(6)$ & $48.3(7)$ & $2.0(5)$ & $-14.9(5)$ & $-23.2(5)$ \\
\hline N006 & $63.9(8)$ & $49.5(7)$ & 66.1(9) & $4.7(6)$ & $-24.4(7)$ & $-27.5(6)$ \\
\hline $\mathrm{C} 007$ & $48.6(8)$ & $43.8(7)$ & $41.9(7)$ & $-6.8(6)$ & $-5.9(6)$ & $-21.9(6)$ \\
\hline $\mathrm{C} 008$ & $54.8(8)$ & $44.6(8)$ & $41.8(7)$ & $-3.6(6)$ & $-7.8(6)$ & $-25.3(6)$ \\
\hline C009 & $57.3(8)$ & $40.5(7)$ & $45.6(8)$ & $-5.1(6)$ & $-11.6(6)$ & $-22.7(6)$ \\
\hline $\mathrm{C} 00 \mathrm{~A}$ & $50.3(8)$ & 41.1(7) & $45.5(8)$ & $-5.2(6)$ & $-9.6(6)$ & $-20.8(6)$ \\
\hline C00B & $45.4(7)$ & $41.2(7)$ & $42.5(7)$ & $-2.7(6)$ & $-6.7(6)$ & $-20.2(6)$ \\
\hline $\mathrm{C} 00 \mathrm{C}$ & $57.1(8)$ & $42.6(7)$ & $42.9(7)$ & $-6.6(6)$ & $-4.5(6)$ & $-25.4(6)$ \\
\hline COOD & 49.1(8) & $40.2(7)$ & $44.1(7)$ & $-3.6(6)$ & $-5.9(6)$ & $-21.7(6)$ \\
\hline $\mathrm{COOE}$ & $59.3(9)$ & $46.0(8)$ & $42.5(8)$ & $-3.0(6)$ & $-9.2(6)$ & $-28.2(7)$ \\
\hline $\mathrm{COOF}$ & $52.0(8)$ & $47.3(8)$ & $44.7(8)$ & $-7.4(6)$ & $-7.1(6)$ & $-23.7(6)$ \\
\hline $\mathrm{C} 00 \mathrm{G}$ & $63.7(10)$ & $54.9(9)$ & $55.5(9)$ & $-6.5(7)$ & $-13.3(7)$ & $-21.4(8)$ \\
\hline $\mathrm{COOH}$ & $65.7(10)$ & $59.6(9)$ & $47.6(8)$ & $-8.3(7)$ & $-5.4(7)$ & $-26.2(8)$ \\
\hline C00I & $85.2(12)$ & $47.8(9)$ & $49.6(9)$ & $-0.4(7)$ & $-13.2(8)$ & $-35.3(8)$ \\
\hline $\mathrm{C} 00 \mathrm{~J}$ & $75.7(11)$ & $70.0(11)$ & $54.9(9)$ & $3.3(8)$ & $-16.1(8)$ & $-44.9(9)$ \\
\hline $\mathrm{COOK}$ & $54.8(9)$ & $60.3(9)$ & $68(1)$ & $-15.5(8)$ & $-11.6(8)$ & $-24.1(8)$ \\
\hline $\mathrm{COOL}$ & $59(1)$ & $51.1(9)$ & $88.1(13)$ & $-16.7(9)$ & $-20.2(9)$ & $-20.0(8)$ \\
\hline $\mathrm{C} 00 \mathrm{M}$ & $91.5(13)$ & $79.7(12)$ & $44.7(9)$ & $-12.3(8)$ & $-3.6(8)$ & $-42.9(11)$ \\
\hline $\mathrm{COON}$ & $69.7(11)$ & $69.6(11)$ & $89.4(14)$ & $-32.3(10)$ & $0.8(10)$ & $-38.3(9)$ \\
\hline $\mathrm{C} 00 \mathrm{O}$ & $85.9(13)$ & $69.0(11)$ & $63.8(10)$ & $-17.2(9)$ & $-18.7(9)$ & $-41.2(10)$ \\
\hline $\mathrm{COOP}$ & $103.8(15)$ & $78.1(12)$ & 48.1(9) & $5.0(9)$ & $-26.5(10)$ & $-51.9(12)$ \\
\hline C00Q & $80.3(12)$ & $61.8(10)$ & $68.8(12)$ & $2.3(9)$ & $-32.9(10)$ & $-26.3(9)$ \\
\hline C00R & $82.1(12)$ & $59.5(10)$ & $76.4(12)$ & $6.1(9)$ & $-40.9(10)$ & $-30.4(9)$ \\
\hline
\end{tabular}




\begin{tabular}{lcllcll} 
C00S & $72.3(12)$ & $77.4(12)$ & $84.4(13)$ & $-13.5(10)$ & $-23.3(10)$ & $-41.2(10)$ \\
C00T & $100.1(15)$ & $60.4(11)$ & $91.3(14)$ & $-15.3(10)$ & $-32.1(12)$ & $-42.7(11)$ \\
C00U & $112.8(16)$ & $64.0(11)$ & $61.4(11)$ & $15.4(9)$ & $-38.6(11)$ & $-44.6(11)$ \\
C00V & $115.1(17)$ & $86.8(14)$ & $58.8(11)$ & $17.3(10)$ & $-24.6(11)$ & $-67.4(14)$ \\
C00W & $85.2(14)$ & $53.1(10)$ & $117.7(18)$ & $-32.1(11)$ & $-24.5(12)$ & $-20.6(10)$ \\
\hline
\end{tabular}

Table S17 Bond Lengths for 5a.

\begin{tabular}{llclll}
\hline Atom & Atom & Length/A & Atom & Atom & Length/A \\
\hline O001 & C00A & $1.2157(18)$ & C009 & C00J & $1.378(2)$ \\
O002 & C00F & $1.3323(19)$ & C00B & C00D & $1.363(2)$ \\
O002 & C00O & $1.439(2)$ & C00C & C00I & $1.492(2)$ \\
N003 & C00C & $1.3694(19)$ & C00E & C00G & $1.377(2)$ \\
N003 & C00D & $1.3991(18)$ & C00E & C00H & $1.373(2)$ \\
N003 & C00E & $1.4364(18)$ & C00G & C00Q & $1.389(2)$ \\
O004 & C00F & $1.2066(19)$ & C00H & C00M & $1.387(2)$ \\
N005 & C00A & $1.3494(19)$ & C00J & C00V & $1.376(3)$ \\
N005 & C00B & $1.4087(18)$ & C00K & C00S & $1.382(3)$ \\
N006 & C009 & $1.331(2)$ & C00L & C00W & $1.387(3)$ \\
N006 & C00R & $1.336(2)$ & C00M & C00P & $1.366(3)$ \\
C007 & C00B & $1.436(2)$ & C00N & C00O & $1.483(3)$ \\
C007 & C00C & $1.383(2)$ & C00P & C00Q & $1.372(3)$ \\
C007 & C00F & $1.460(2)$ & C00R & C00U & $1.366(3)$ \\
C008 & C00D & $1.480(2)$ & C00S & C00T & $1.371(3)$ \\
C008 & C00K & $1.386(2)$ & C00T & C00W & $1.371(3)$ \\
C008 & C00L & $1.374(2)$ & C00U & C00V & $1.370(3)$ \\
C009 & C00A & $1.506(2)$ & & & \\
\hline
\end{tabular}

Table S18 Bond Angles for 5a.

\begin{tabular}{lccccc}
\hline Atom Atom Atom & Angle $^{\circ}$ & Atom & Atom & Atom & Angle $^{\circ}$ \\
\hline C00F O002 C00O & $118.80(13)$ & N003 C00D C008 & $120.98(12)$ \\
C00C N003 C00D & $110.51(12)$ & C00B C00D N003 & $106.73(12)$ \\
C00C N003 C00E & $124.51(12)$ & C00B C00D C008 & $132.29(13)$ \\
C00D N003 C00E & $124.54(12)$ & C00G C00E N003 & $119.39(14)$ \\
C00A N005 C00B & $125.84(12)$ & C00H C00E N003 & $119.80(14)$ \\
C009 N006 C00R & $117.05(15)$ & C00H C00E C00G & $120.80(15)$ \\
C00B C007 C00F & $124.55(13)$ & O002 C00F C007 & $113.09(13)$ \\
C00C C007 C00B & $107.32(12)$ & O004 C00F O002 & $122.65(14)$ \\
C00C C007 C00F & $128.01(13)$ & O004 C00F C007 & $124.25(14)$ \\
C00K C008 C00D & $120.81(14)$ & C00E C00G C00Q & $119.44(18)$
\end{tabular}




\begin{tabular}{lllll} 
C00L C008 C00D & $120.40(14)$ & C00E C00H C00M & $119.20(17)$ \\
C00L C008 C00K & $118.78(15)$ & C00V C00J C009 & $118.41(17)$ \\
N006 C009 C00A & $117.10(13)$ & C00S C00K C008 & $120.46(17)$ \\
N006 C009 C00J & $123.17(14)$ & C008 C00L C00W & $120.56(17)$ \\
C00J C009 C00A & $119.73(14)$ & C00P C00M C00H & $120.29(18)$ \\
O001 C00A N005 & $125.02(13)$ & O002 C00O C00N & $107.78(16)$ \\
O001 C00A C009 & $121.30(13)$ & C00M C00P C00Q & $120.52(17)$ \\
N005 C00A C009 & $113.67(12)$ & C00P C00Q C00G & $119.72(18)$ \\
N005 C00B C007 & $123.93(13)$ & N006 C00R C00U & $123.64(18)$ \\
C00D C00B N005 & $127.82(13)$ & C00T C00S C00K & $120.26(17)$ \\
C00D C00B C007 & $108.24(12)$ & C00W C00T C00S & $119.67(17)$ \\
N003 C00C C007 & $107.18(12)$ & C00R C00U C00V & $118.57(17)$ \\
N003 C00C C00I & $121.09(13)$ & C00U C00V C00J & $119.16(18)$ \\
C007 C00C C00I & $131.70(14)$ & C00T C00W C00L & $120.26(19)$ \\
\hline
\end{tabular}

Table S19 Torsion Angles for 5a.

\begin{tabular}{|c|c|c|c|c|c|c|c|c|c|}
\hline A & B & $\mathrm{C}$ & D & & A & B & $\mathrm{C}$ & D & \\
\hline N003 & COOE & $\mathrm{COOG}$ & COOQ & $-178.16(15)$ & COOD & N003 & $\mathrm{COOE}$ & $\mathrm{COOH}$ & \\
\hline & & & & 179. & & & $\mathrm{COOK}$ & COOS & (17) \\
\hline $\mathrm{N}$ & & & & & & & & COOW & 178.68(19) \\
\hline NOO & & & $\mathrm{C} 008$ & & & & $\mathrm{C} 00 \mathrm{C}$ & $\mathrm{C} 007$ & 173.61(13) \\
\hline N006 & $\mathrm{COC}$ & $\mathrm{COO}$ & O001 & $-174.78(15)$ & & & $\mathrm{C} 00 \mathrm{C}$ & $\mathrm{COOI}$ & \\
\hline N006 & $\mathrm{CO}$ & $\mathrm{C} 00$ & & & & & C00D & $\mathrm{C} 008$ & \\
\hline N00 & & $\mathrm{C} 00 \mathrm{~J}$ & & & & & & & \\
\hline & & & & & & & & & \\
\hline & & & & & & & & & \\
\hline $\mathrm{COO}$ & & & $\mathrm{C} 008$ & -178 . & Covi & & & & -10 \\
\hline $\mathrm{COO}$ & & $\mathrm{COOS}$ & $\mathrm{CO}$ & & $\mathrm{COOF}$ & & $\mathrm{C} 00 \mathrm{~B}$ & 005 & \\
\hline $\mathrm{COC}$ & & $\mathrm{CO}$ & & & $0 \mathrm{~F}$ & & $\mathrm{COOB}$ & $0 \mathrm{D}$ & 17 \\
\hline $\mathrm{C} 009$ & N006 & $\mathrm{CO}$ & $\mathrm{COOU}$ & 0 & $\mathrm{COOF}$ & 007 & $\mathrm{C} 00 \mathrm{C}$ & N003 & -17 \\
\hline $\mathrm{C} 009$ & $\mathrm{COOJ}$ & $\mathrm{COOV}$ & & & $\mathrm{COOF}$ & $\mathrm{C} 007$ & $\mathrm{C} 00 \mathrm{C}$ & $\mathrm{COOI}$ & \\
\hline COOA & N & $\mathrm{COOB}$ & $\mathrm{C} 007$ & -132.6 & $\mathrm{COOG}$ & $\mathrm{COOE}$ & $\mathrm{COOH}$ & $\mathrm{COOM}$ & \\
\hline $\mathrm{COOA}$ & N & $\mathrm{COOB}$ & CO0D & & $\mathrm{COOH}$ & & $\mathrm{C} 00 \mathrm{G}$ & C00Q & \\
\hline $\mathrm{COOH}$ & C009 & $\mathrm{C} 00 \mathrm{~J}$ & $\mathrm{COOV}$ & -178.47 & $\mathrm{COOH}$ & & $\mathrm{COOP}$ & C00Q & \\
\hline $\mathrm{COOI}$ & & $\mathrm{C} 00 \mathrm{~A}$ & & & & & $\mathrm{C} 00 \mathrm{~A}$ & & \\
\hline $\mathrm{COOI}$ & N00 & $\mathrm{COOA}$ & $\mathrm{C} 009$ & $174.36(13)$ & $\mathrm{COOJ}$ & $\mathrm{C} 009$ & $\mathrm{C} 00 \mathrm{~A}$ & N005 & $-175.41(15)$ \\
\hline $\mathrm{COOB}$ & $\mathrm{C} 007$ & $\mathrm{COOC}$ & N003 & $-0.29(16)$ & $\mathrm{COOK}$ & $\mathrm{C} 008$ & C00D & N003 & \\
\hline $\mathrm{COOB}$ & $\mathrm{C} 007$ & $\mathrm{COOC}$ & $\mathrm{COOI}$ & $177.73(17)$ & $\mathrm{COOK}$ & $\mathrm{C} 008$ & C00D & $\mathrm{COOB}$ & $-119.47(19)$ \\
\hline $\mathrm{COOB}$ & $\mathrm{C} 007$ & $\mathrm{COOF}$ & $\mathrm{O} 002$ & $174.61(14)$ & $\mathrm{COOK}$ & $\mathrm{C} 008$ & $\mathrm{COOL}$ & COOW & $-0.2(3)$ \\
\hline
\end{tabular}




$\begin{array}{llllll}\text { C00B C007 C00F O004 } & -6.2(3) & \text { C00K C00S C00T C00W } & -0.5(4) \\ \text { C00C N003 C00D C008 } & 178.02(13) & \text { C00L C008 C00D N003 } & -117.36(18) \\ \text { C00C N003 C00D C00B } & -1.25(17) & \text { C00L C008 C00D C00B } & 61.7(2) \\ \text { C00C N003 C00E C00G } & 71.4(2) & \text { C00L C008 C00K C00S } & -0.9(3) \\ \text { C00C N003 C00E C00H } & -107.99(18) & \text { C00M C00P C00Q C00G } & -0.7(3) \\ \text { C00C C007 C00B N005 } & 178.56(13) & \text { C00O O002 C00F O004 } & -3.6(3) \\ \text { C00C C007 C00B C00D } & -0.48(17) & \text { C00O O002 C00F C007 } & 175.59(15) \\ \text { C00C C007 C00F O002 } & -9.9(2) & \text { C00R N006 C009 C00A } & 178.43(16) \\ \text { C00C C007 C00F O004 } & 169.36(16) & \text { C00R N006 C009 C00J } & -0.5(3) \\ \text { C00D N003 C00C C007 } & 0.95(17) & \text { C00R C00U C00V C00J } & -0.5(4) \\ \text { C00D N003 C00C C00I } & -177.32(14) & \text { C00S C00T C00W C00L } & -0.6(4) \\ \text { C00D N003 C00E C00G } & -116.91(17) & & & \end{array}$

Table S20 Hydrogen Atom Coordinates $\left(\AA \times 10^{4}\right)$ and Isotropic Displacement Parameters $\left(\AA^{2} \times 10^{3}\right)$ for 5 a.

\begin{tabular}{ccccc}
\hline Atom & $x$ & $y$ & $z$ & $\mathrm{U}(\mathrm{eq})$ \\
\hline H005 & 1219 & 8490 & 3047 & 56 \\
H00G & 5224 & 3716 & 7153 & 70 \\
H00H & 1613 & 6497 & 8270 & 69 \\
H00A & 3591 & 2883 & 6034 & 88 \\
H00B & 1999 & 3376 & 5885 & 88 \\
H00C & 2475 & 3437 & 7117 & 88 \\
H00J & 3886 & 10639 & 1163 & 76 \\
H00K & 5116 & 6875 & 6173 & 71 \\
H00L & 1108 & 9402 & 6000 & 77 \\
H00M & 2224 & 5672 & 10338 & 83 \\
H00D & 672 & 2280 & 2957 & 105 \\
H00E & 391 & 2676 & 4270 & 105 \\
H00F & 1923 & 1900 & 3739 & 105 \\
H00I & 1719 & 3842 & 2083 & 80 \\
H00N & 218 & 4620 & 2689 & 80 \\
H00P & 4275 & 3837 & 10815 & 87 \\
H00Q & 5795 & 2863 & 9238 & 85 \\
H00R & -339 & 11324 & 88 & 85 \\
H00S & 5541 & 8427 & 6917 & 87 \\
H00T & 3746 & 10438 & 7251 & 92 \\
H00U & 824 & 12654 & -1145 & 93 \\
H00V & 2982 & 12308 & -622 & 98 \\
H00W & 1529 & 10916 & 6809 & \\
\hline
\end{tabular}




\section{Copies of ${ }^{1} \mathrm{H}$ and ${ }^{13} \mathrm{C}$ NMR spectra}

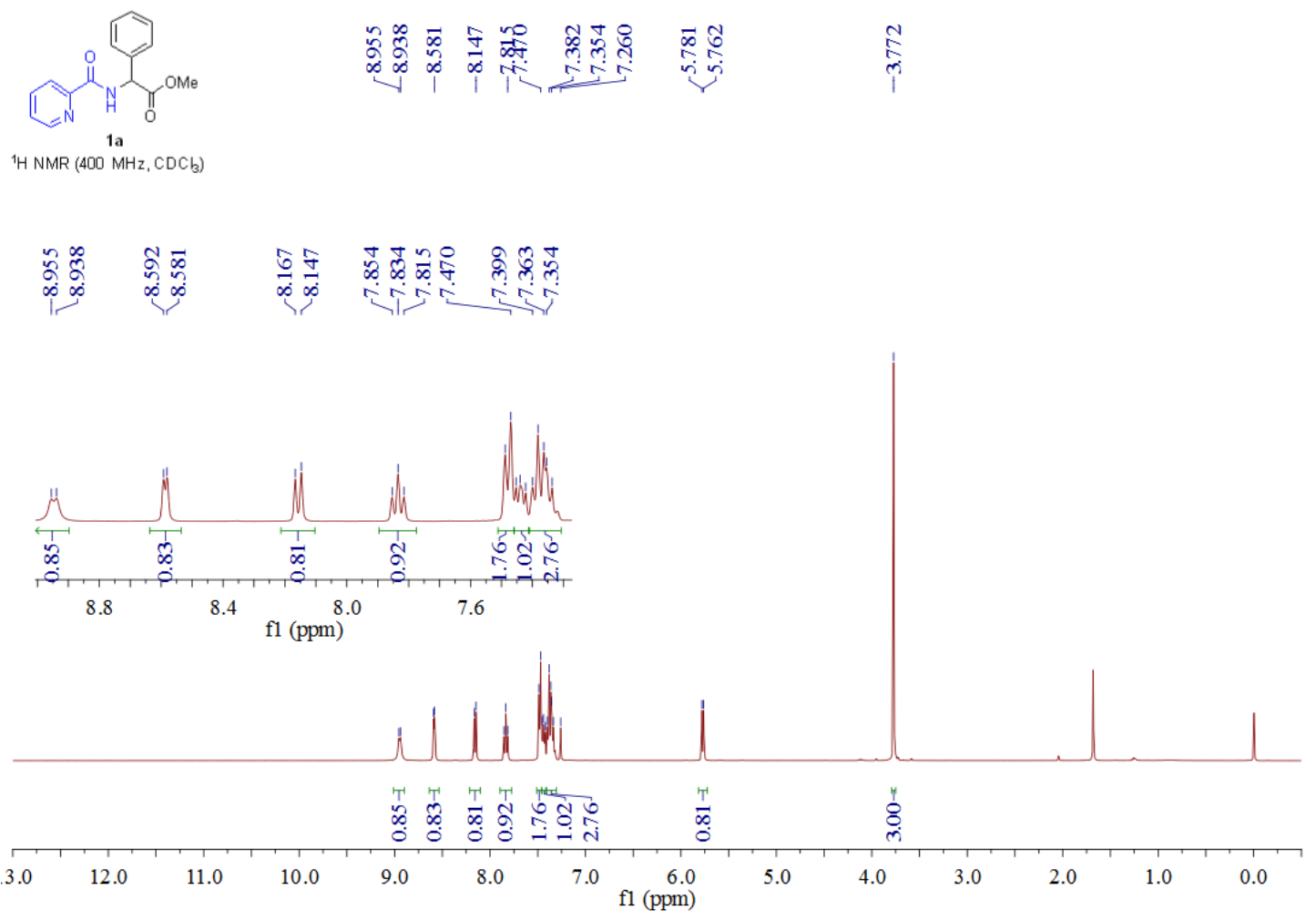

${ }^{1} \mathrm{H}$ NMR spectra for compound $\mathbf{1 a}$ (using $\mathrm{CDCl}_{3}$ as solvent)

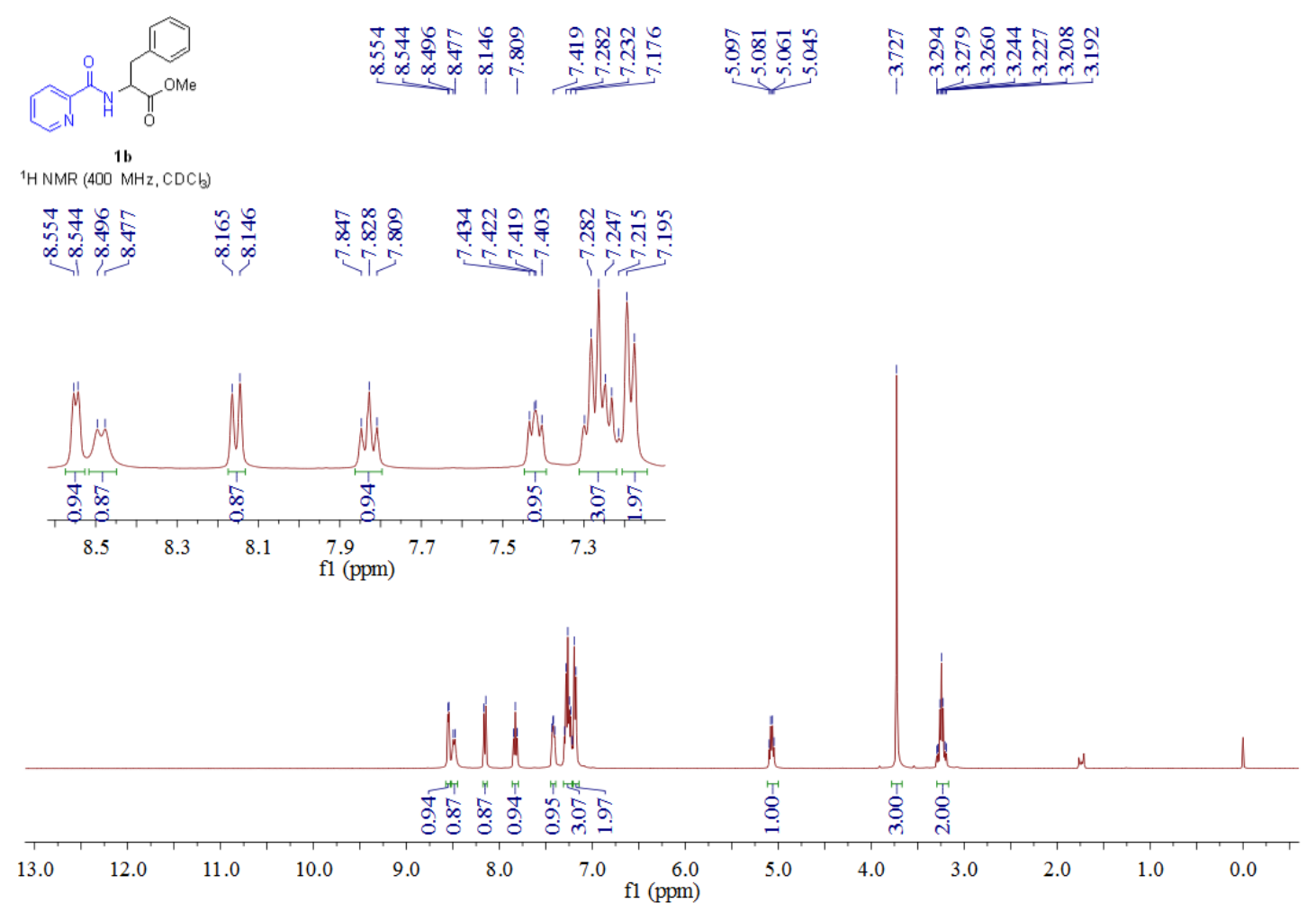

${ }^{1} \mathrm{H}$ NMR spectra for compound $\mathbf{1 b}$ (using $\mathrm{CDCl}_{3}$ as solvent) 


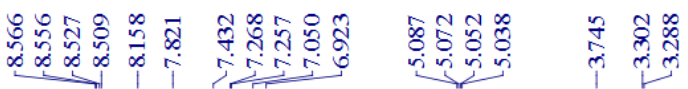

${ }^{1} \mathrm{H} N M R(400 \mathrm{MHz}, \mathrm{CDCb})$

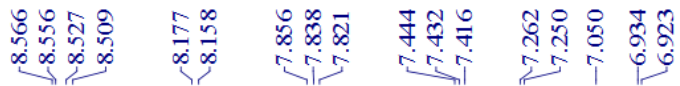
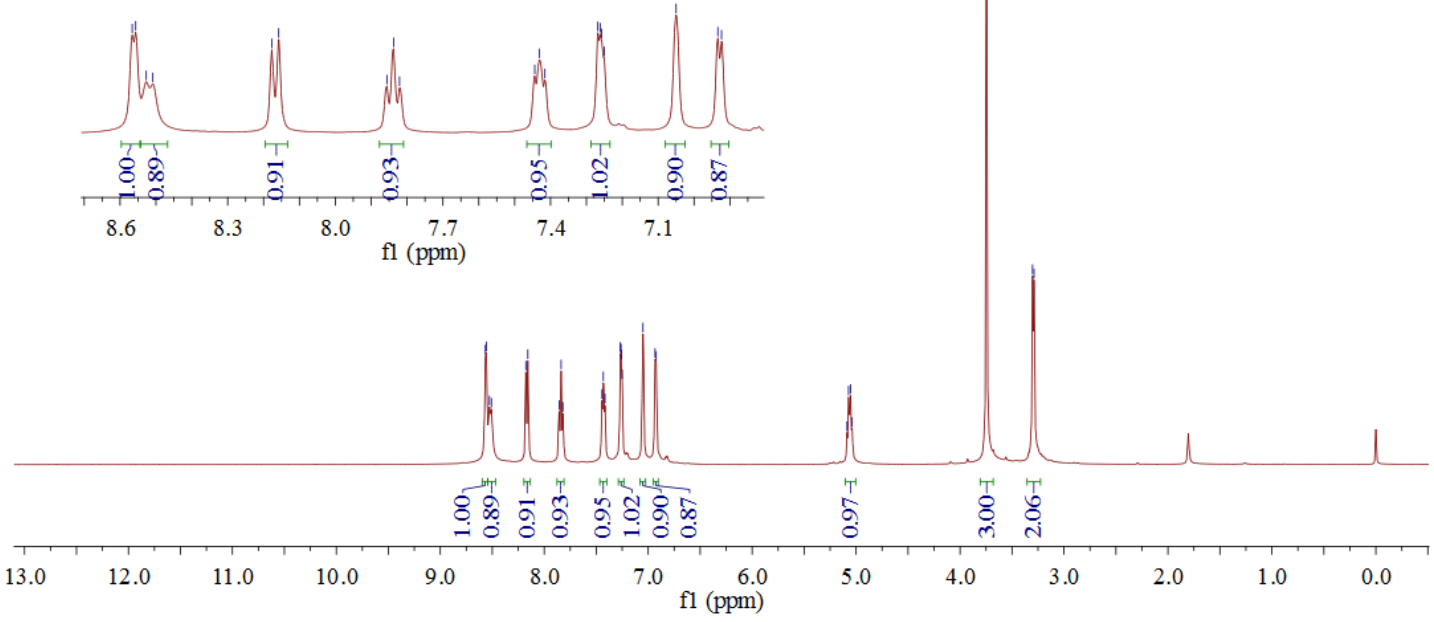

${ }^{1} \mathrm{H}$ NMR spectra for compound $\mathbf{1 c}$ (using $\mathrm{CDCl}_{3}$ as solvent)

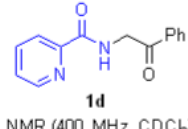

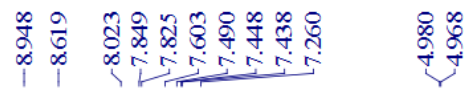

${ }^{1} \mathrm{H}$ NMR (400 MHz, $\mathrm{CDCb}$ )
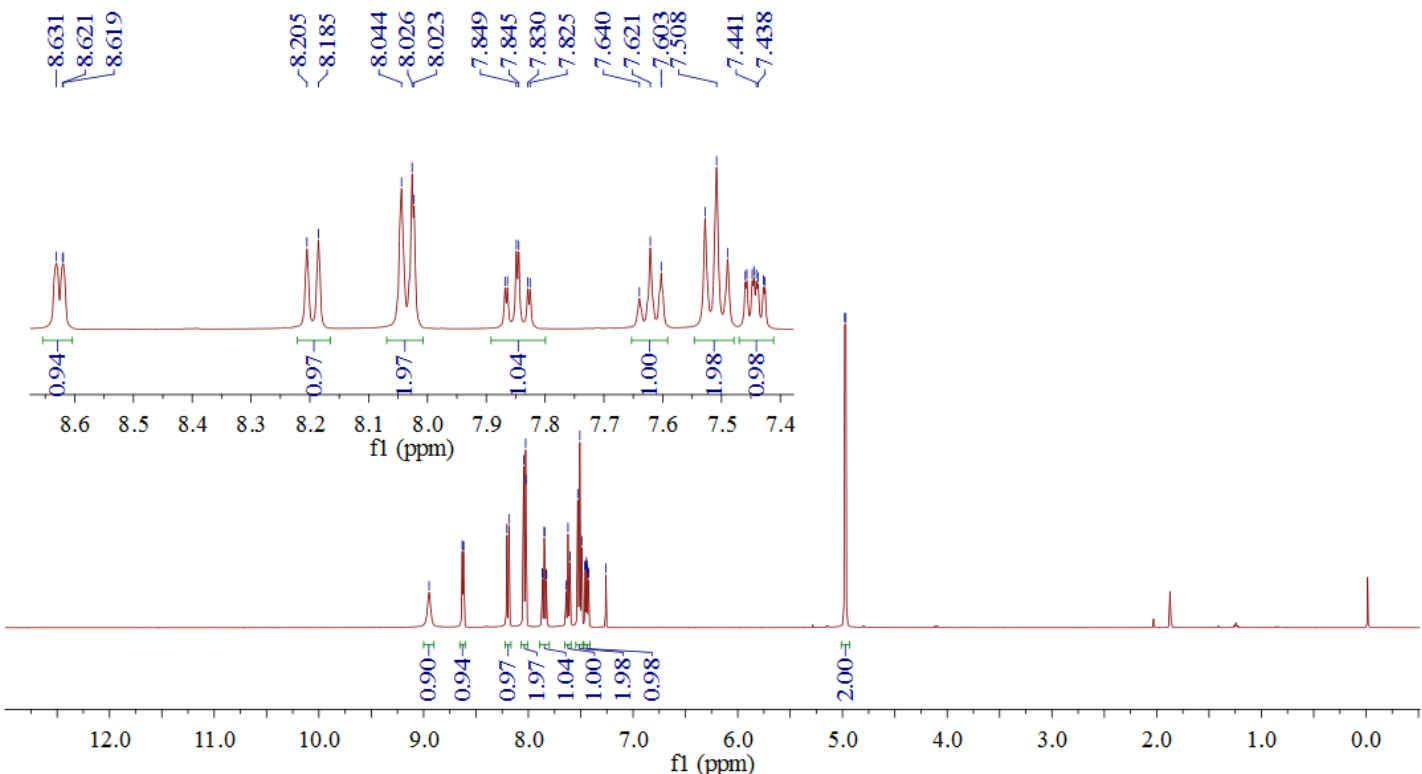


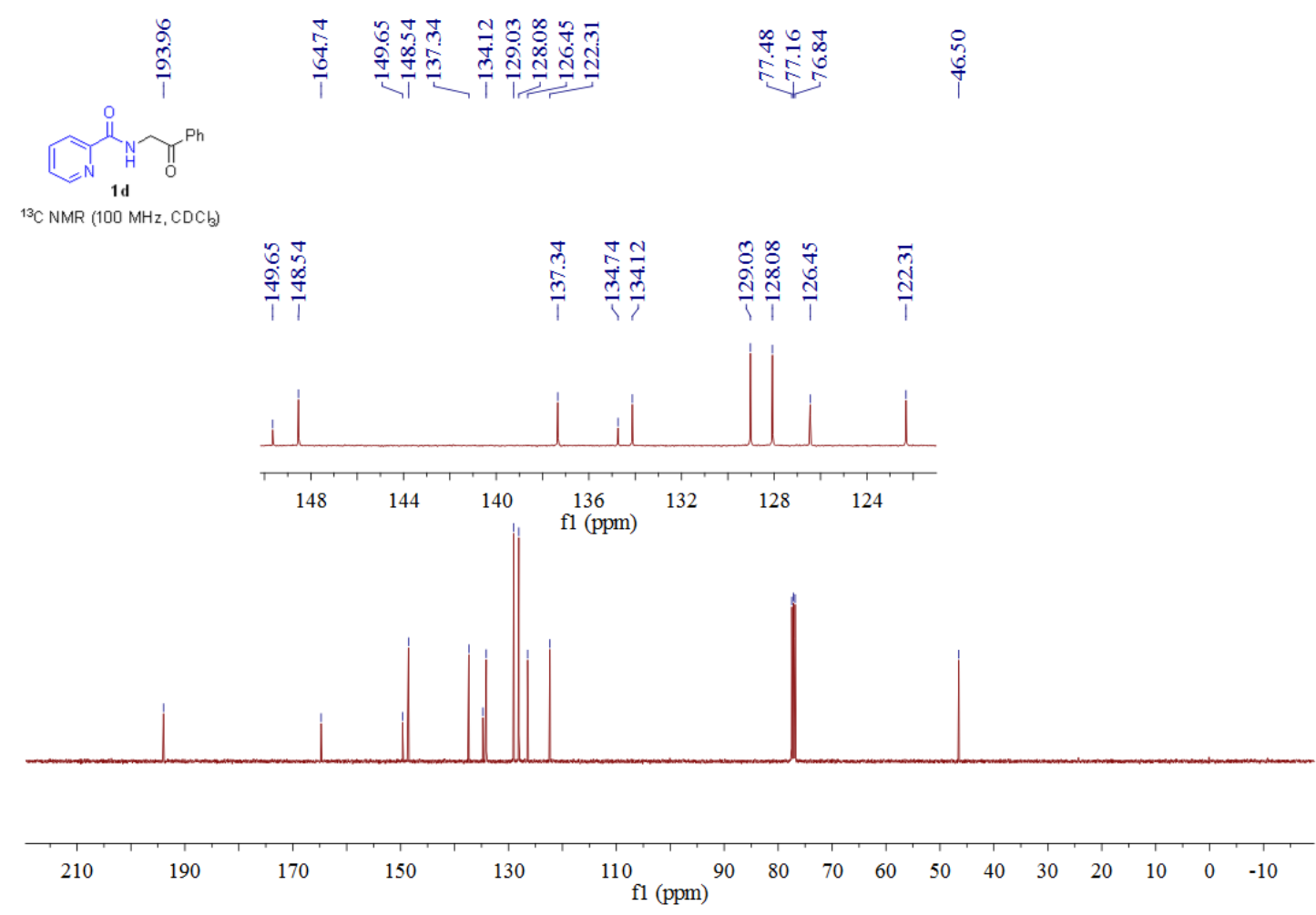

${ }^{1} \mathrm{H}$ NMR and ${ }^{13} \mathrm{C}$ NMR spectra for compound $\mathbf{1 d}$ (using $\mathrm{CDCl}_{3}$ as solvent)

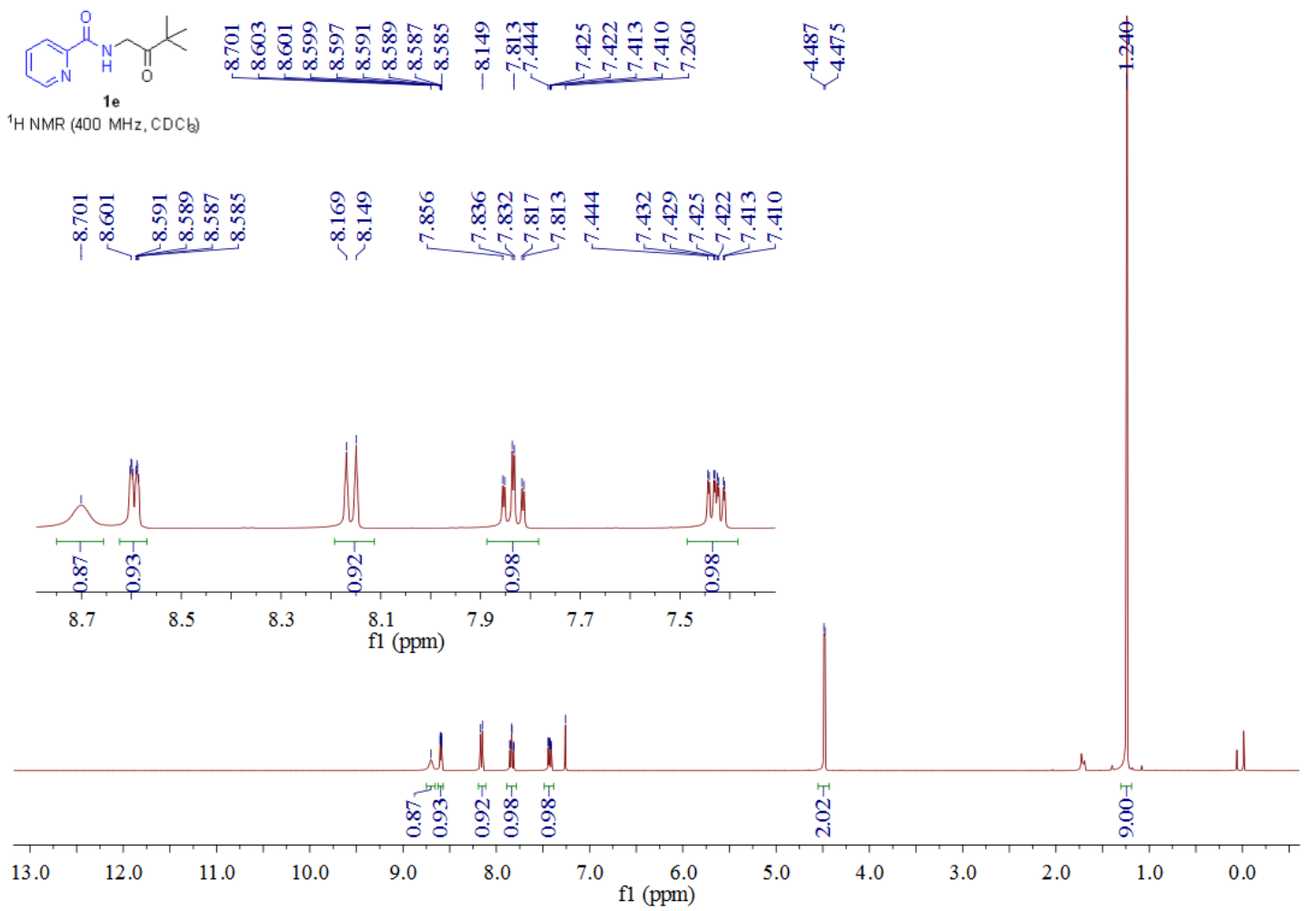



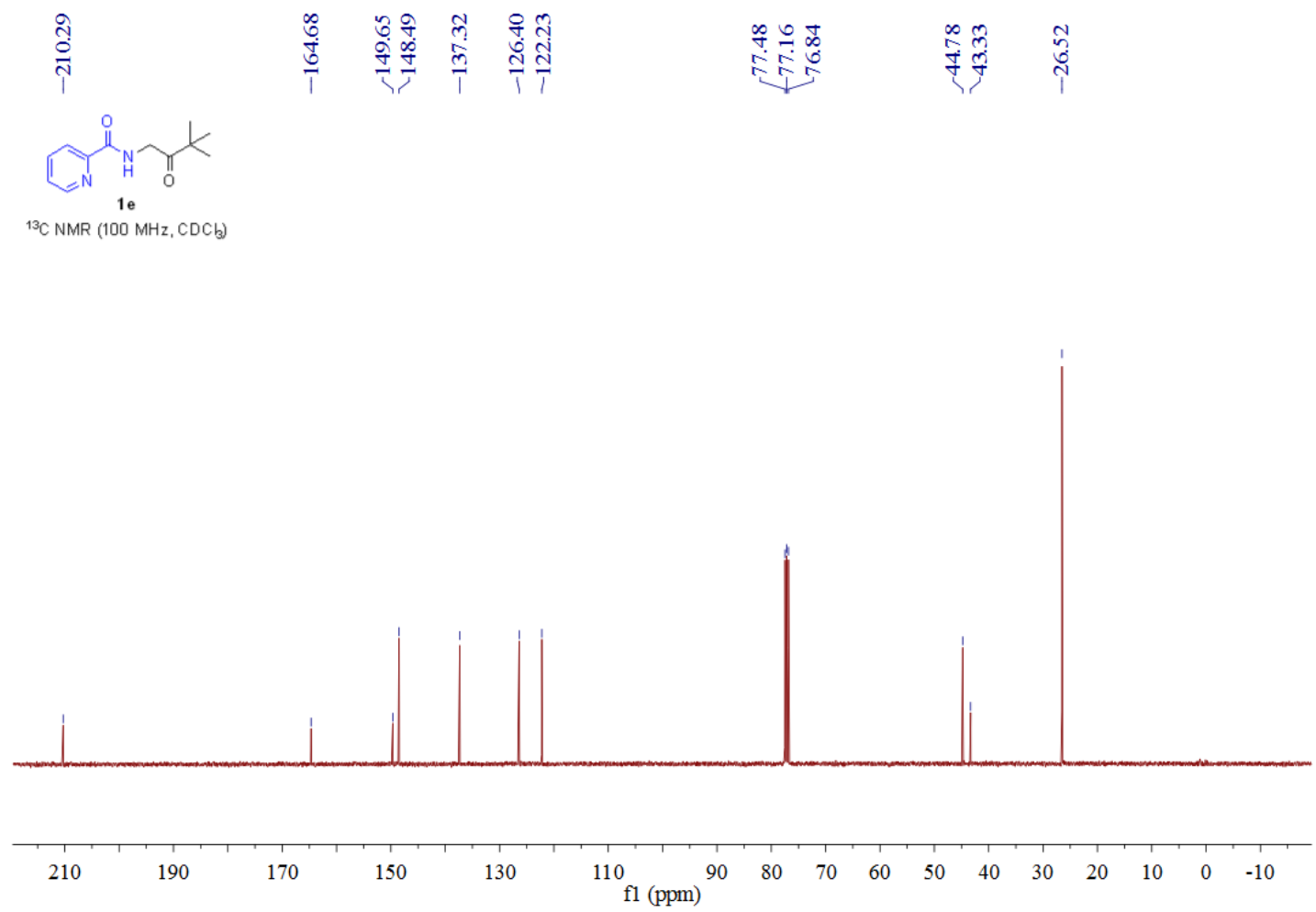

${ }^{1} \mathrm{H}$ NMR and ${ }^{13} \mathrm{C}$ NMR spectra for compound 1e (using $\mathrm{CDCl}_{3}$ as solvent)

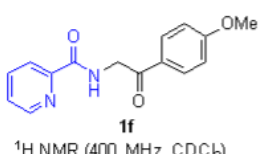

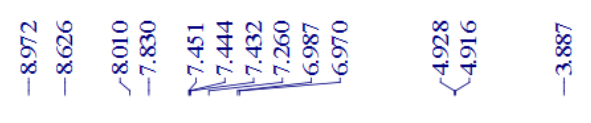

${ }^{1} \mathrm{H}$ NMR $(400 \mathrm{MHz}, \mathrm{CDC} b)$

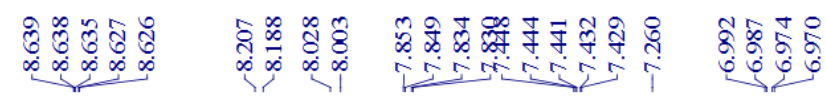

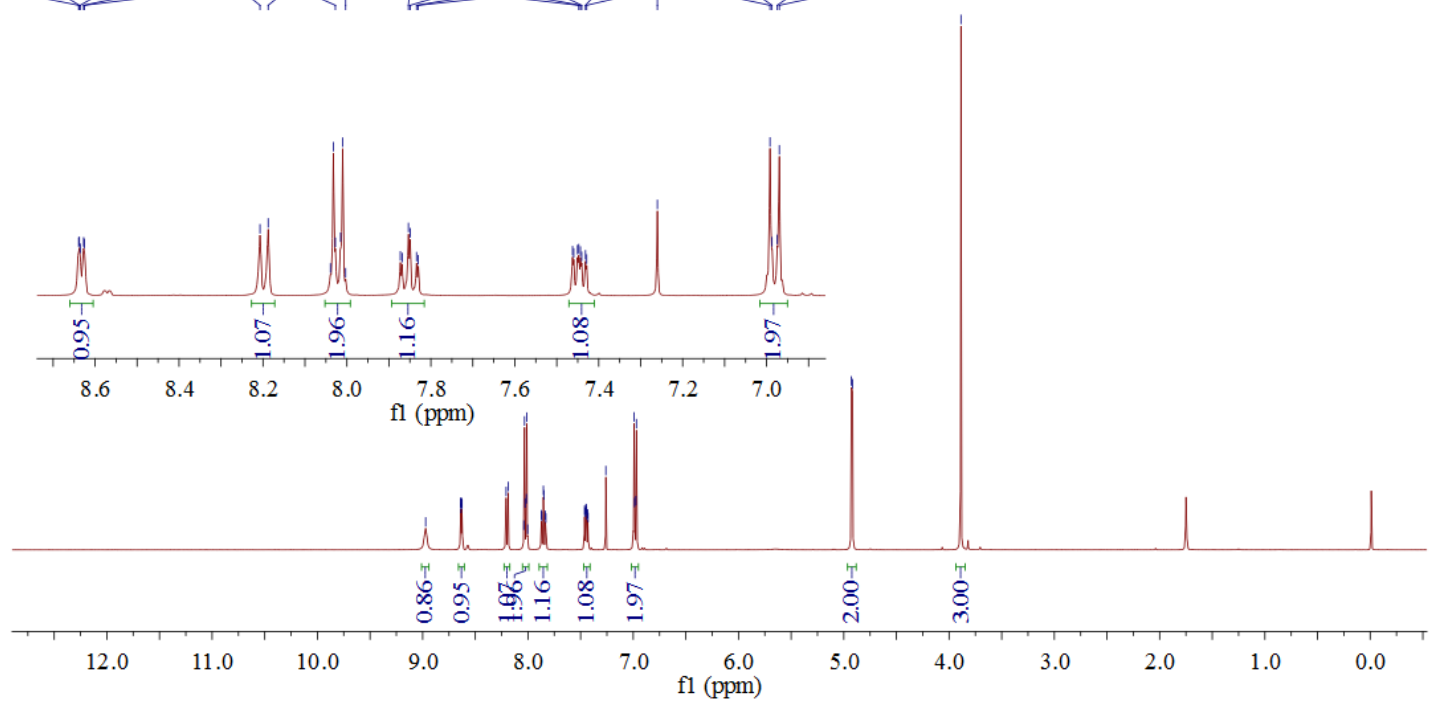



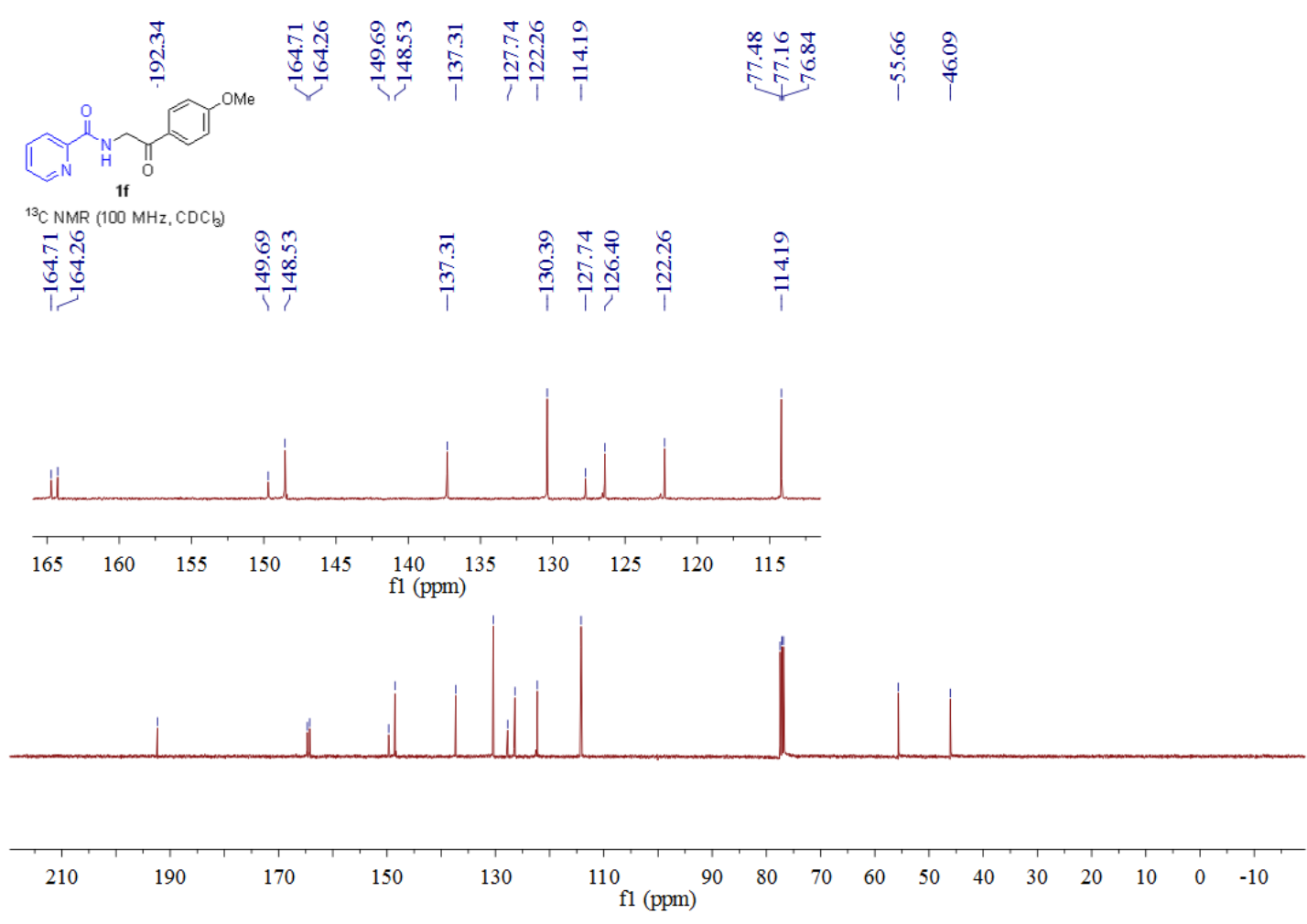

${ }^{1} \mathrm{H}$ NMR and ${ }^{13} \mathrm{C}$ NMR spectra for compound $\mathbf{1 f}$ (using $\mathrm{CDCl}_{3}$ as solvent)

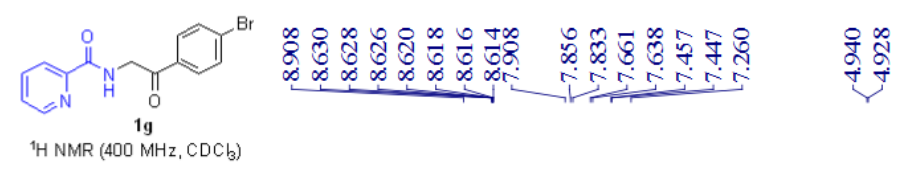

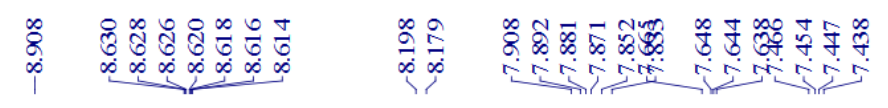

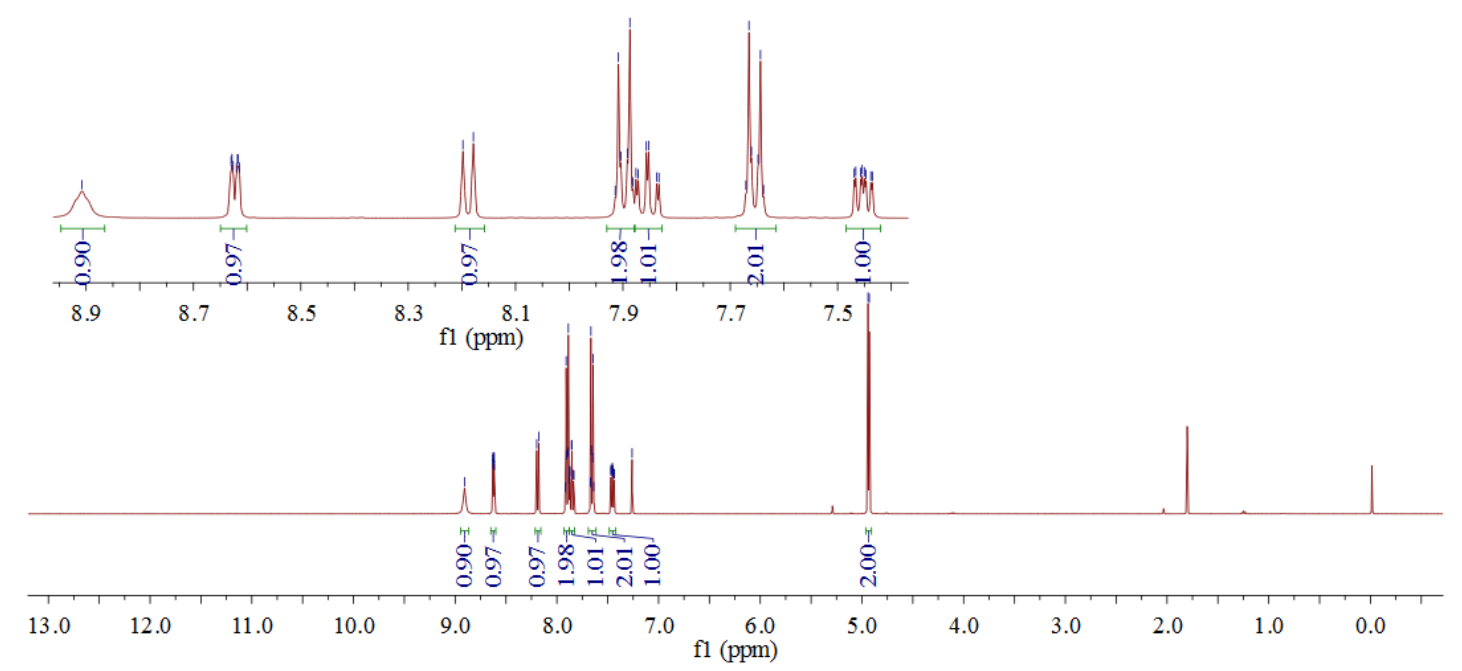



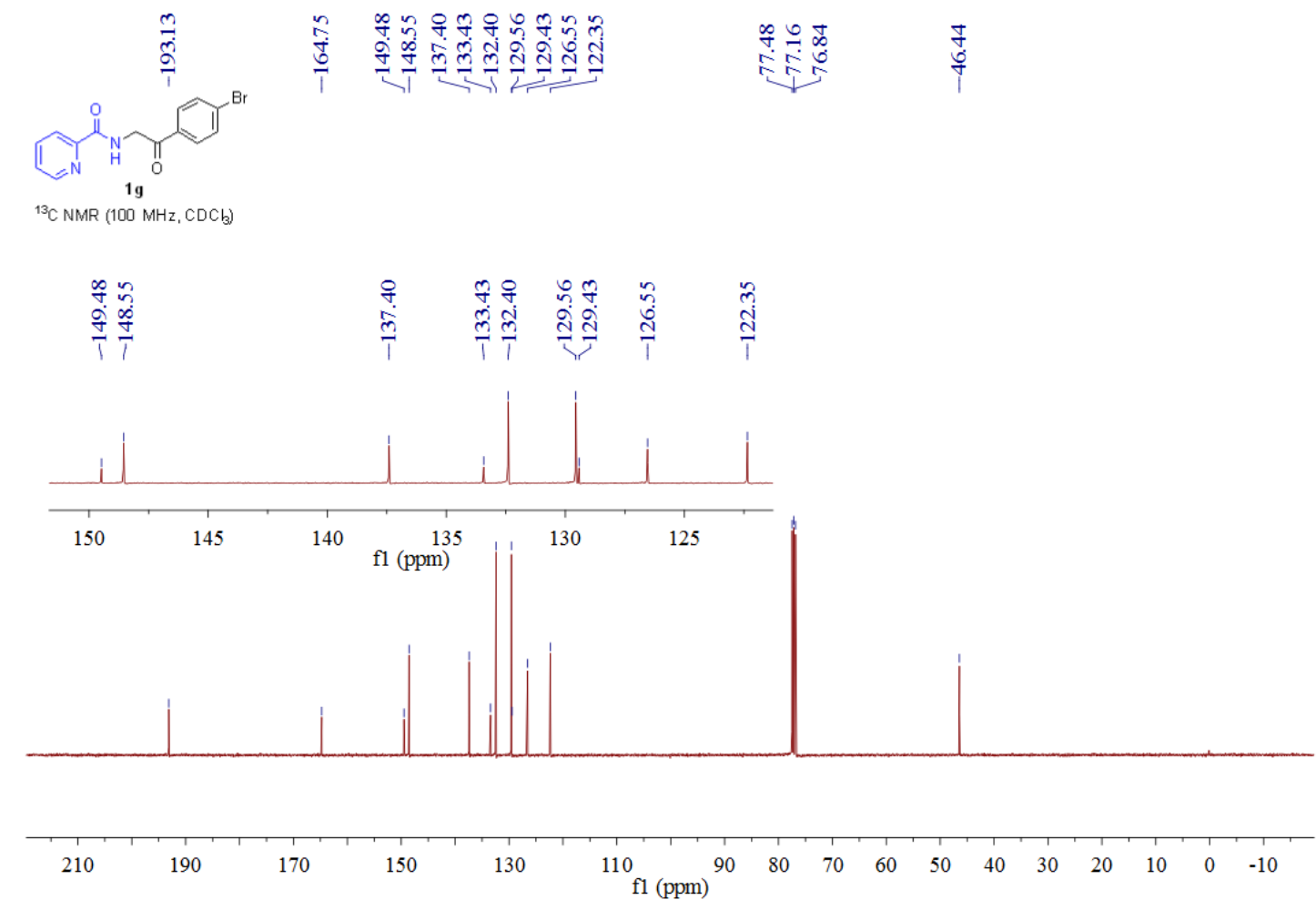

${ }^{1} \mathrm{H}$ NMR and ${ }^{13} \mathrm{C}$ NMR spectra for compound $\mathbf{1 g}$ (using $\mathrm{CDCl}_{3}$ as solvent)
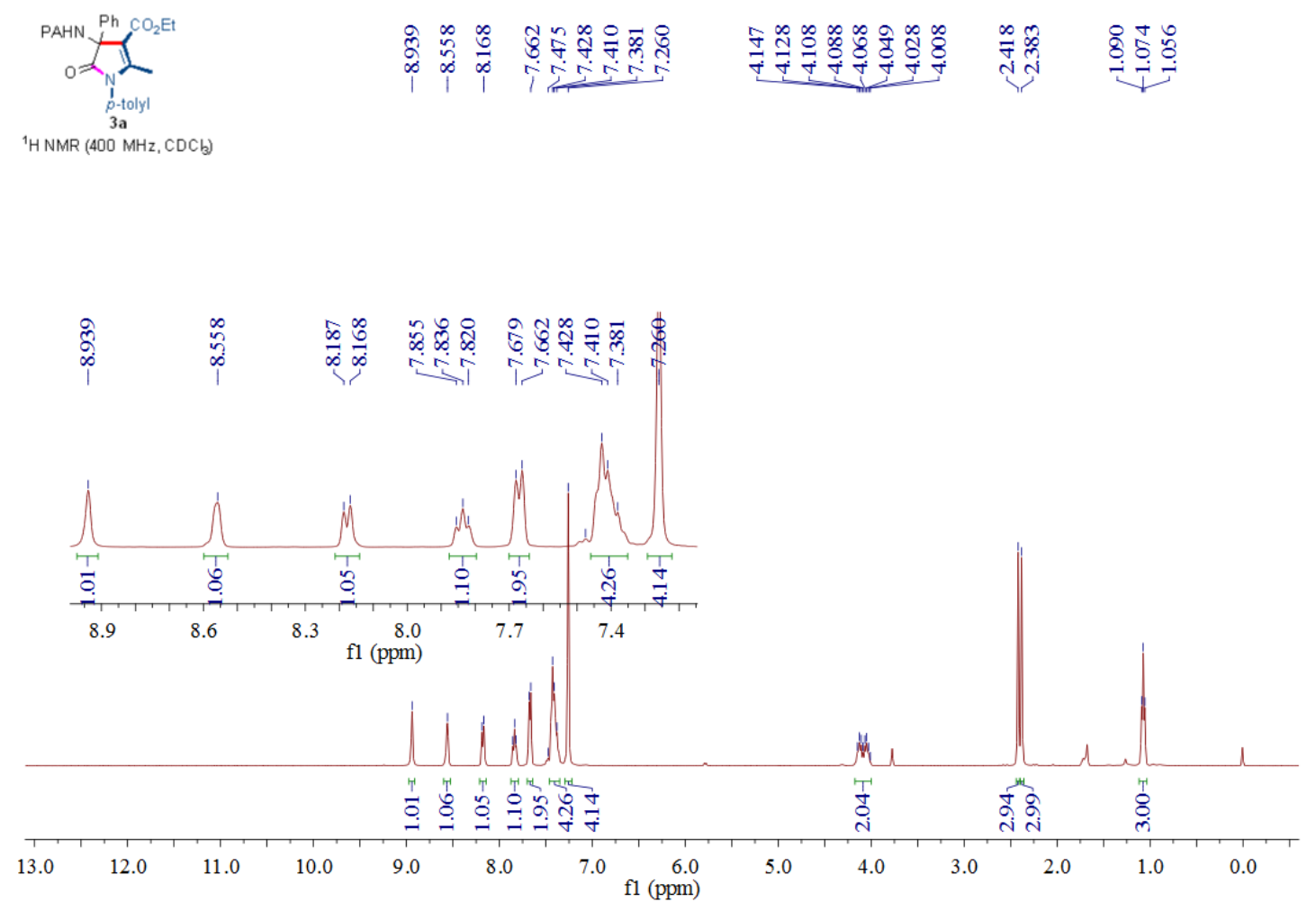

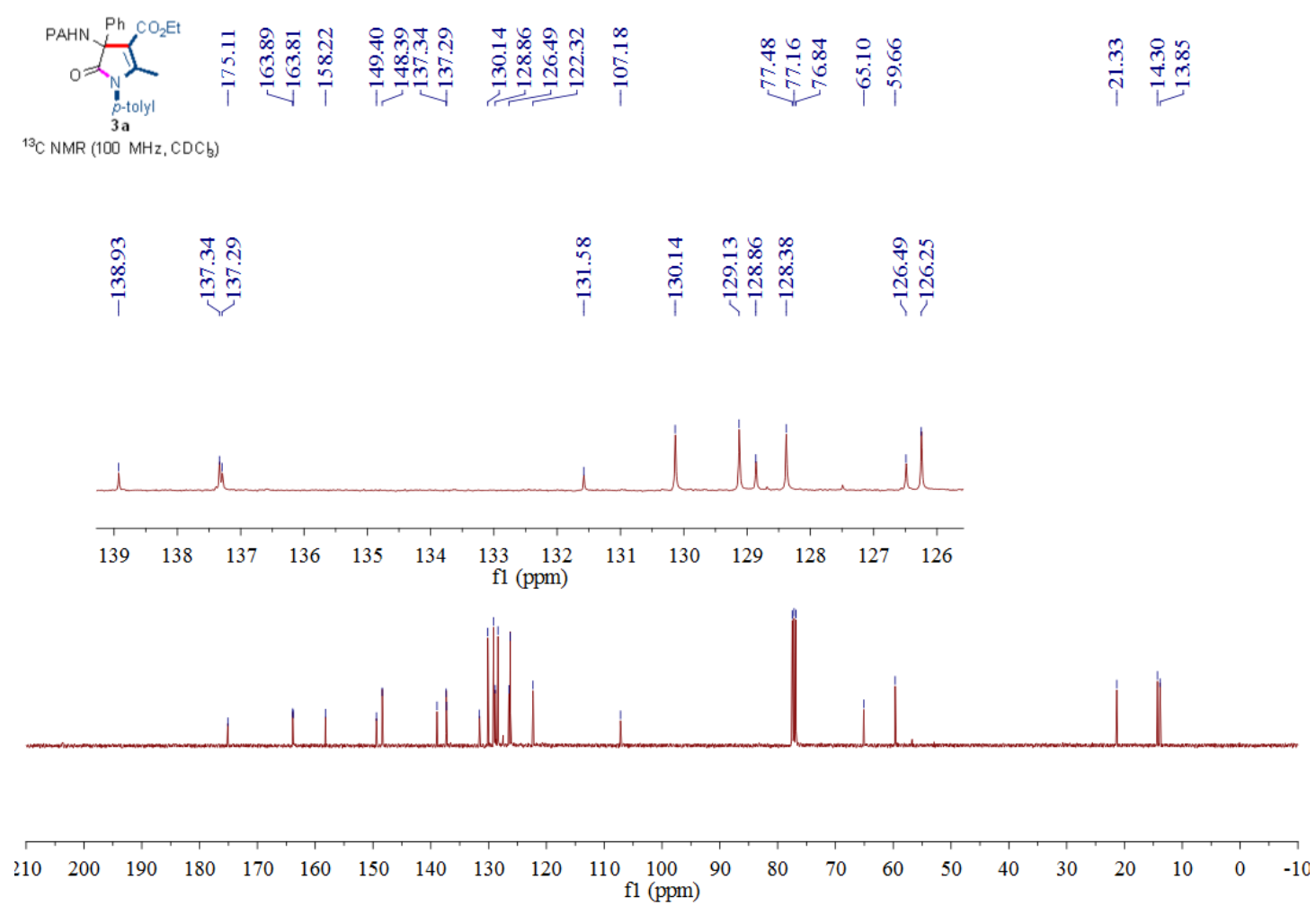

${ }^{1} \mathrm{H}$ NMR and ${ }^{13} \mathrm{C}$ NMR spectra for compound 3a (using $\mathrm{CDCl}_{3}$ as solvent)

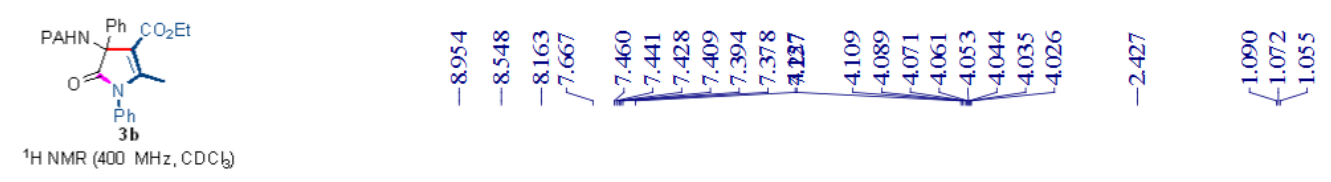

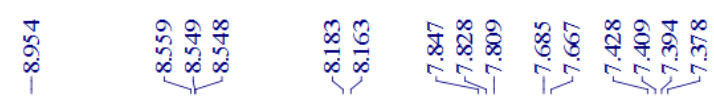
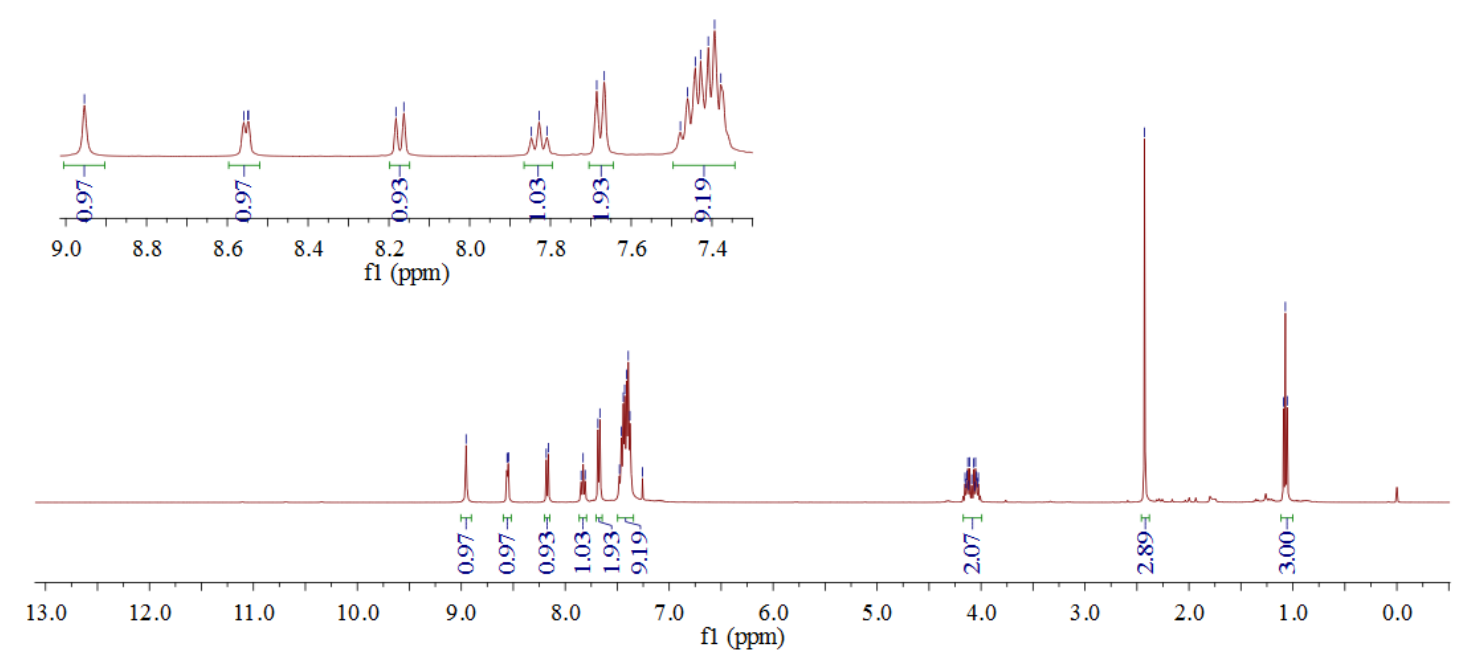


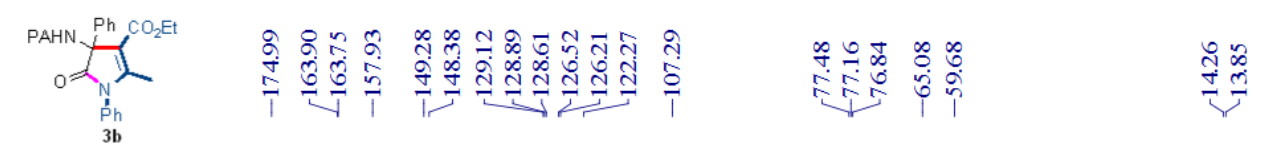

${ }^{13} \mathrm{C}$ NMR $(100 \mathrm{MHz}, \mathrm{CDCb})$

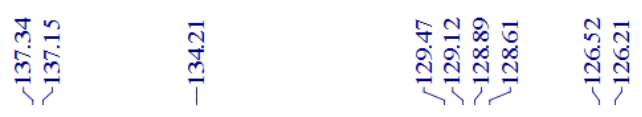

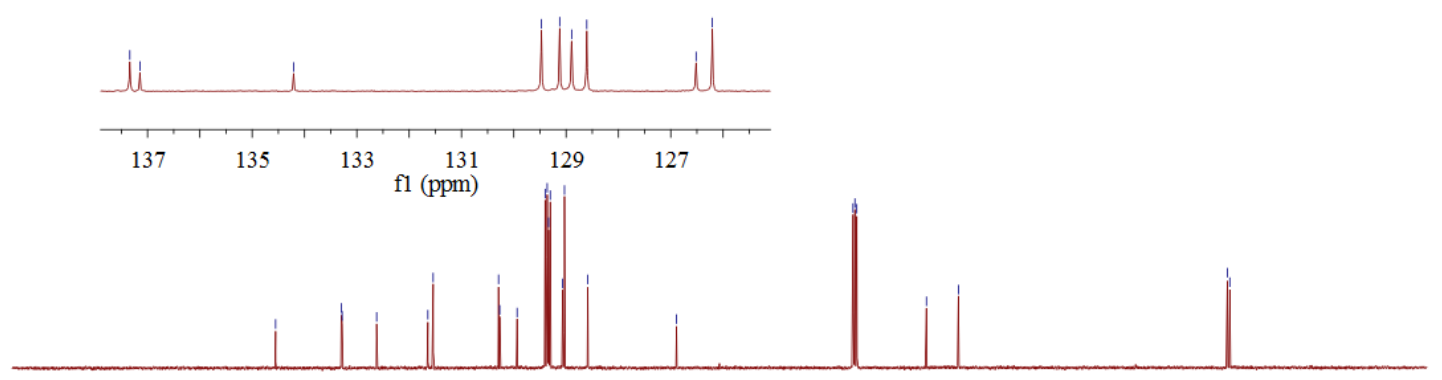

\begin{tabular}{rlllllllllllllllll}
\hline 210 & 190 & 170 & 150 & 130 & 110 & 90 & 80 & 70 & 60 & 50 & 40 & 30 & 20 & 10 & 0 & -10
\end{tabular}

${ }^{1} \mathrm{H}$ NMR and ${ }^{13} \mathrm{C}$ NMR spectra for compound $\mathbf{3 b}$ (using $\mathrm{CDCl}_{3}$ as solvent)

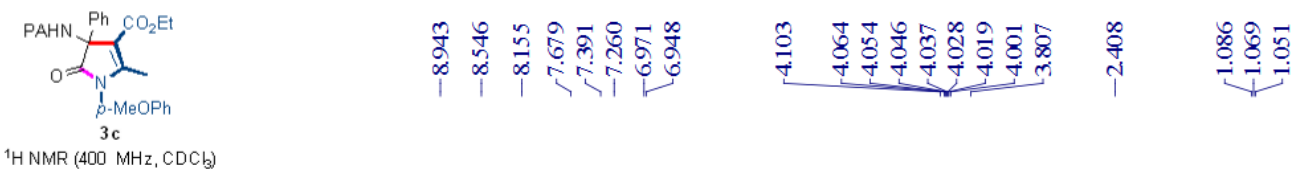

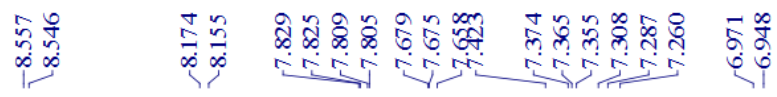

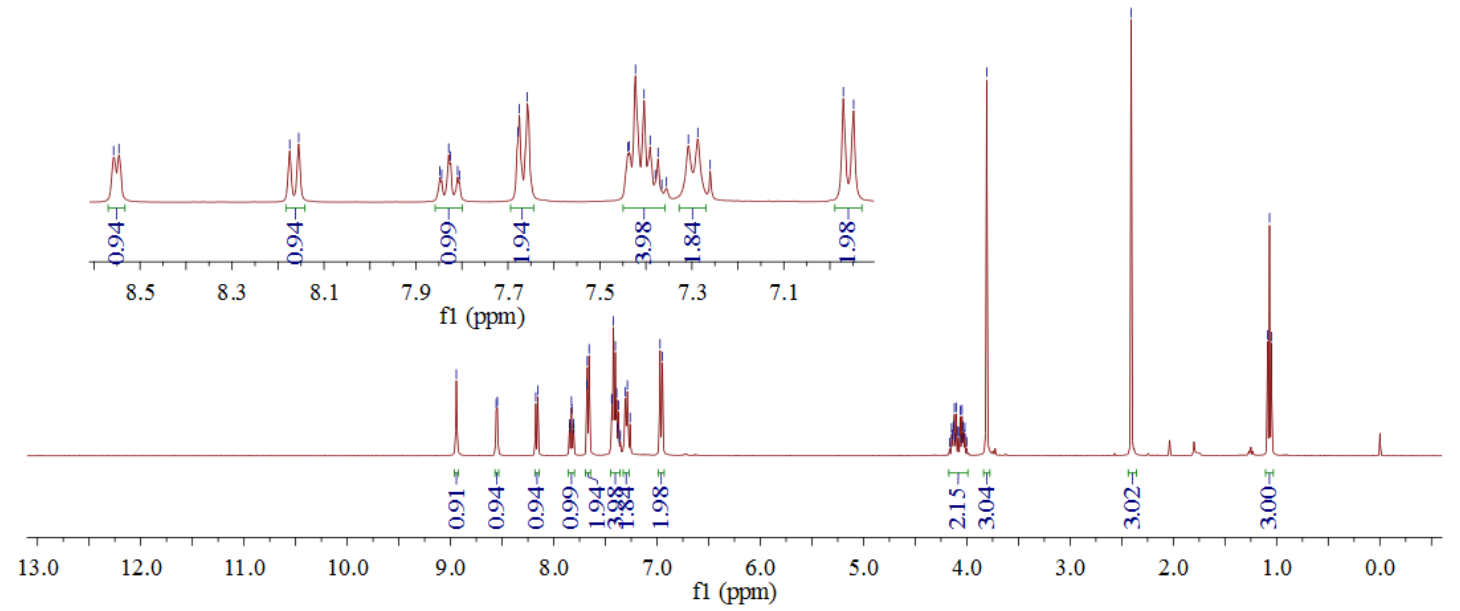




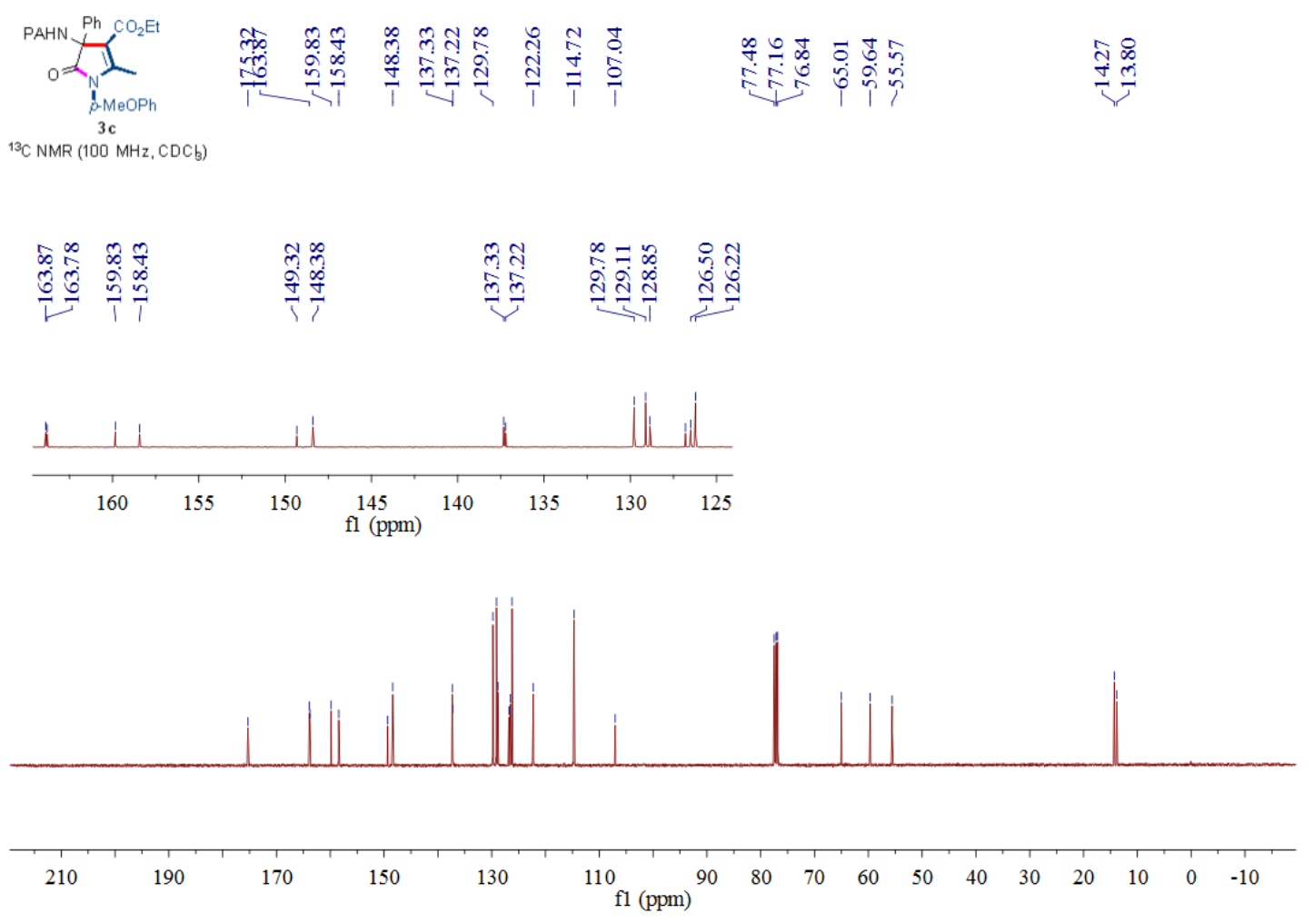

${ }^{1} \mathrm{H}$ NMR and ${ }^{13} \mathrm{C}$ NMR spectra for compound $3 \mathrm{c}$ (using $\mathrm{CDCl}_{3}$ as solvent)

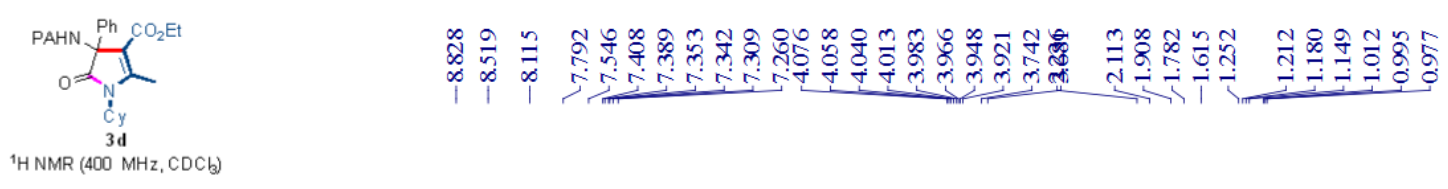

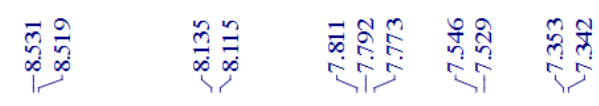

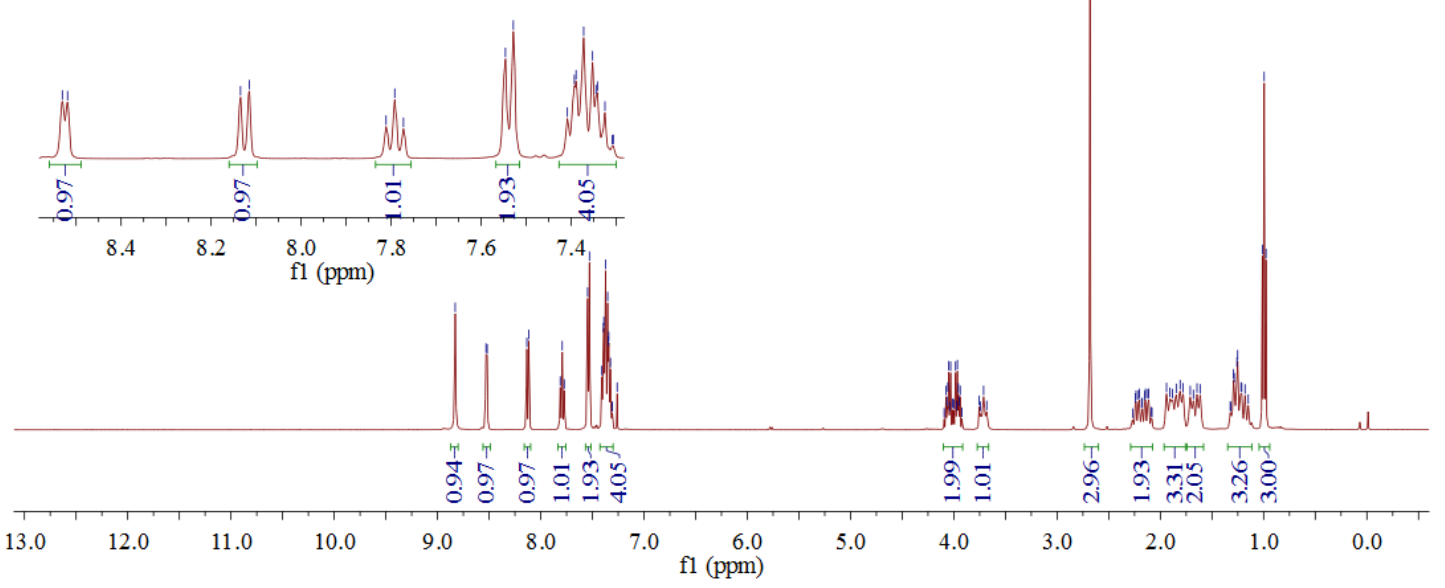




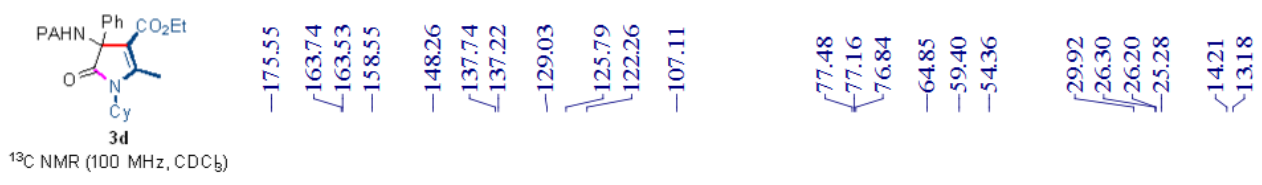

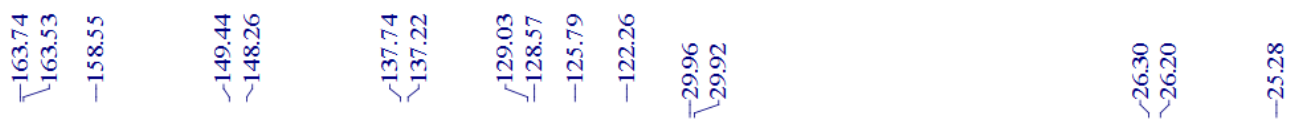
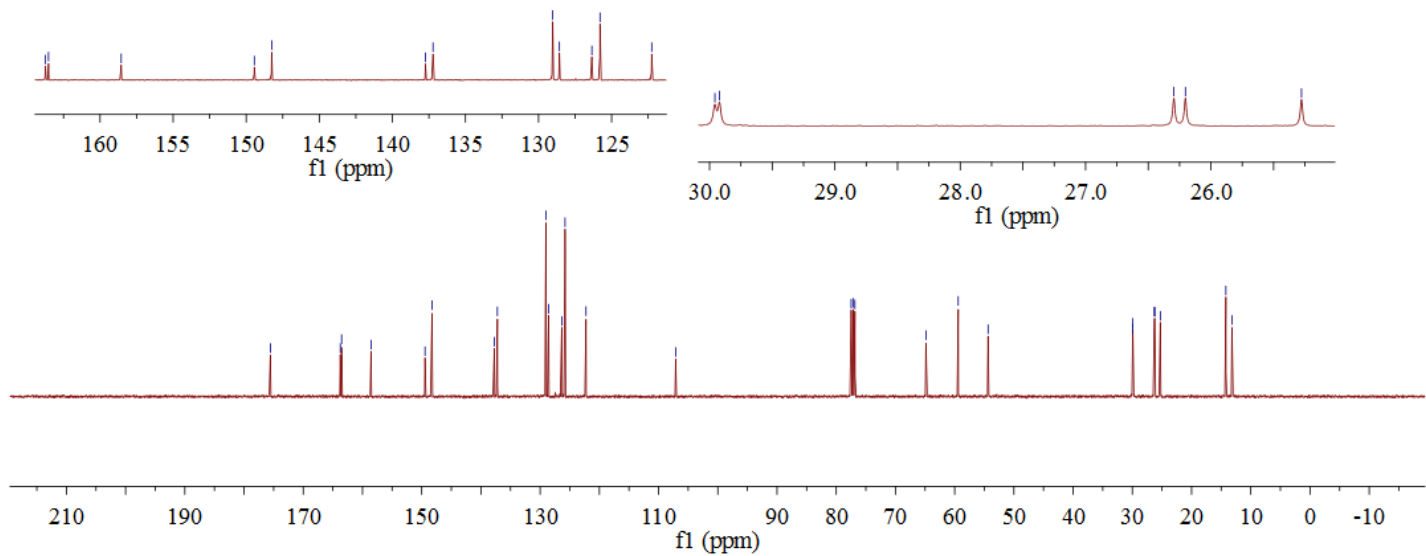

${ }^{1} \mathrm{H}$ NMR and ${ }^{13} \mathrm{C}$ NMR spectra for compound $\mathbf{3 d}$ (using $\mathrm{CDCl}_{3}$ as solvent)

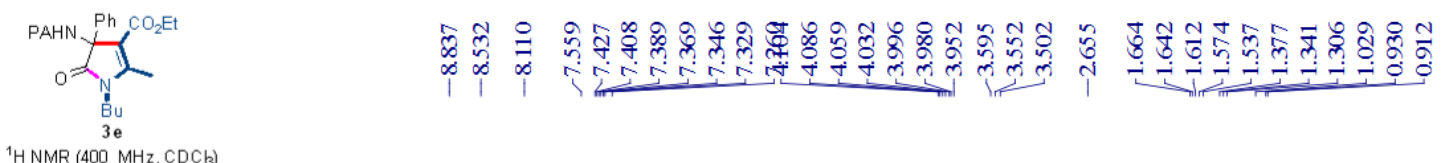

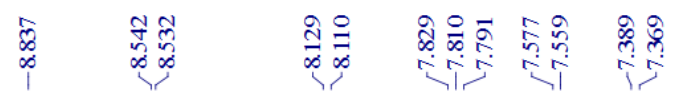
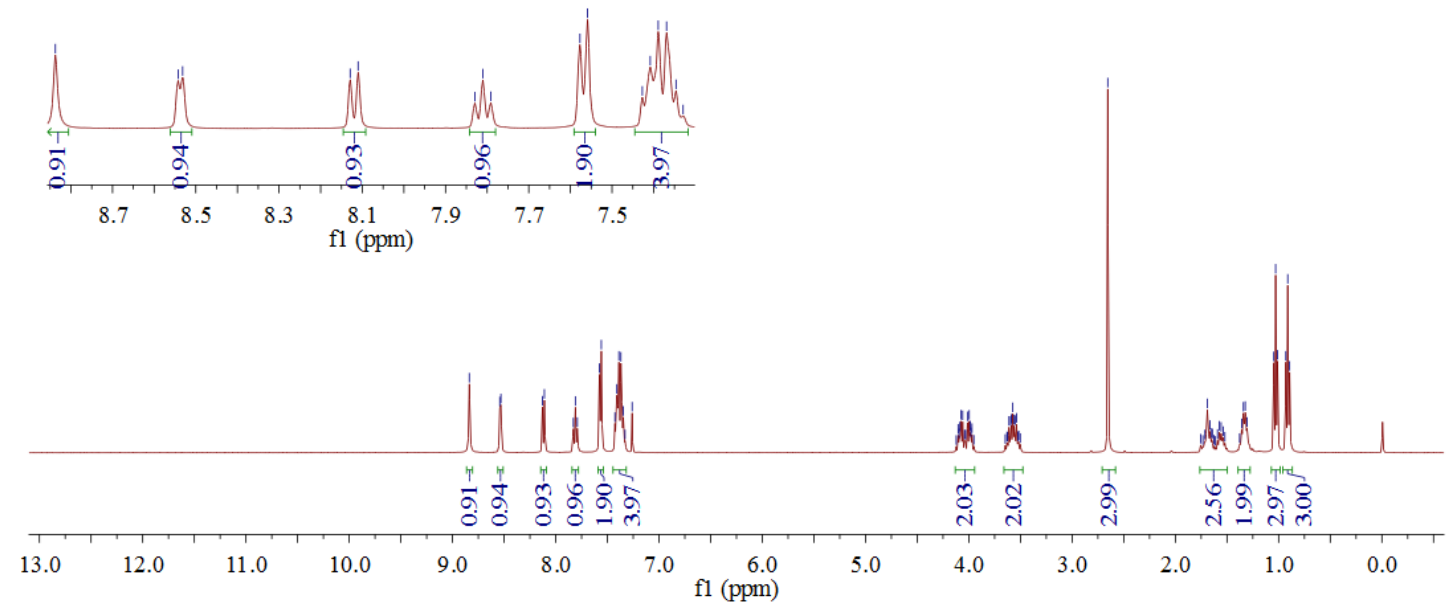


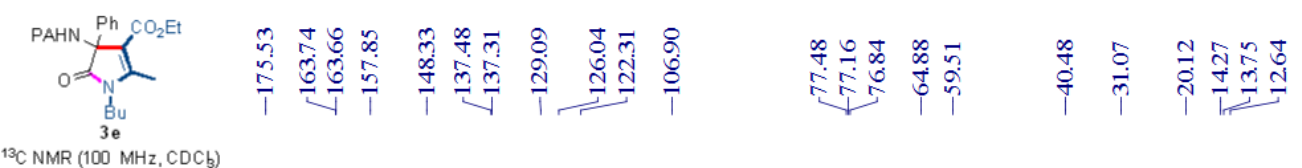

${ }^{13} \mathrm{C}$ NMR $(100 \mathrm{MHz}, \mathrm{CDCb})$

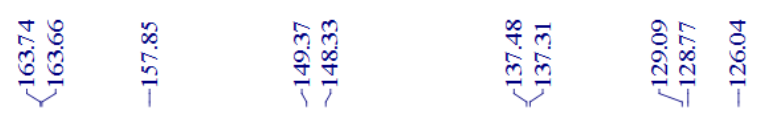

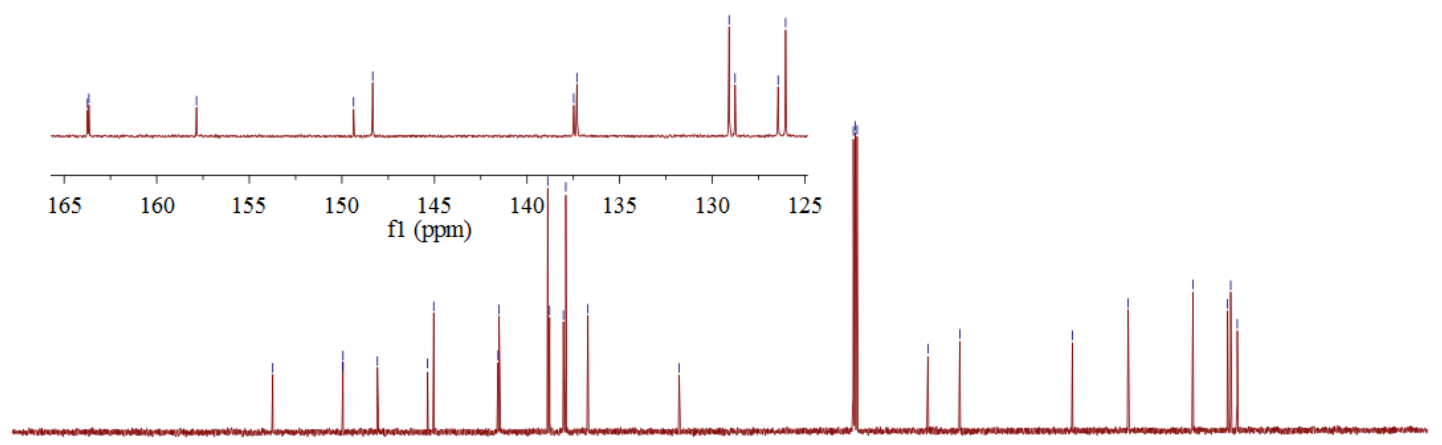

\begin{tabular}{llllllllllllllllllllll}
\hline 210 & 190 & 170 & 150 & 130 & 110 & 90 & 80 & 70 & 60 & 50 & 40 & 30 & 20 & 10 & 0 & -10
\end{tabular}

${ }^{1} \mathrm{H}$ NMR and ${ }^{13} \mathrm{C}$ NMR spectra for compound $3 \mathbf{e}$ (using $\mathrm{CDCl}_{3}$ as solvent)

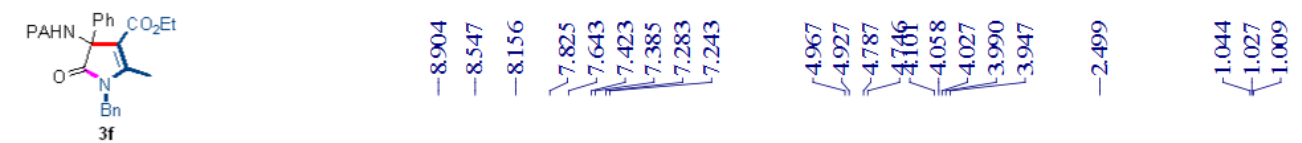

${ }^{1} \mathrm{HNMR}(400 \mathrm{MHz}, \mathrm{CDCl})$

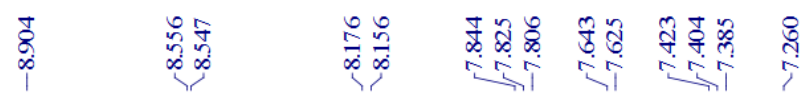
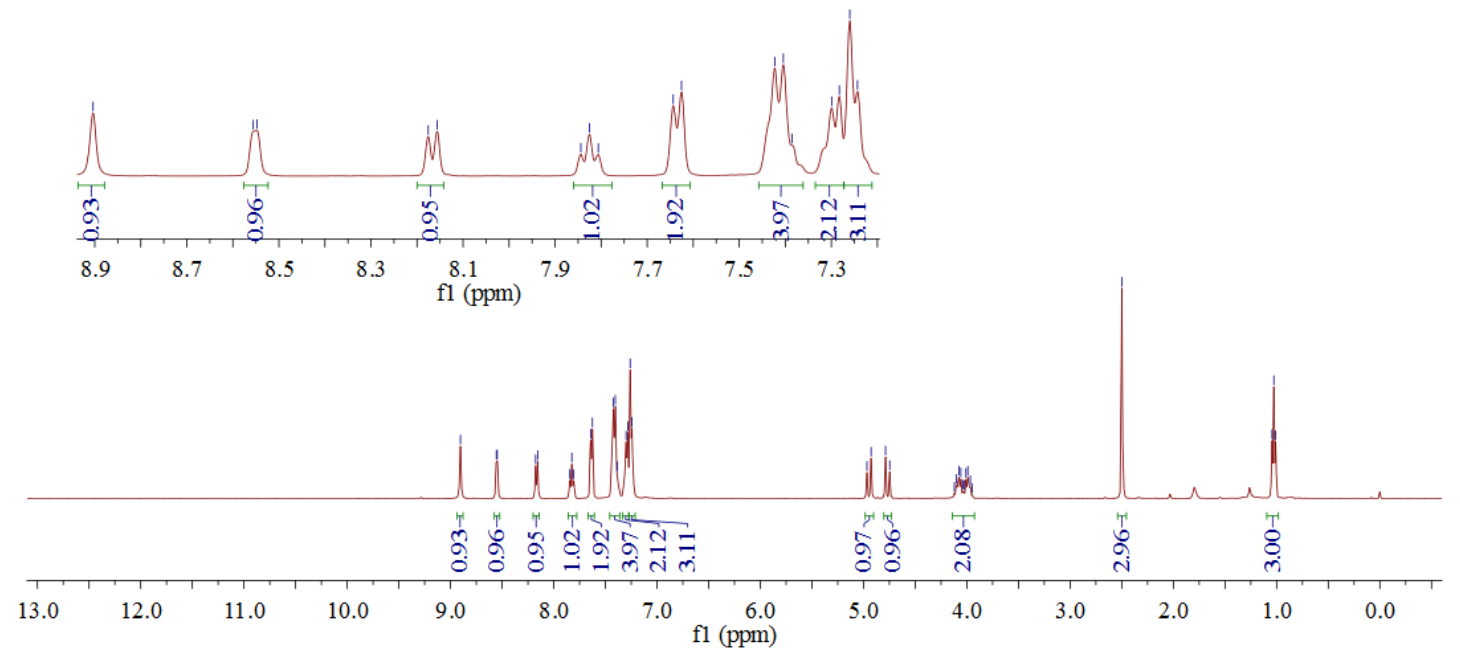


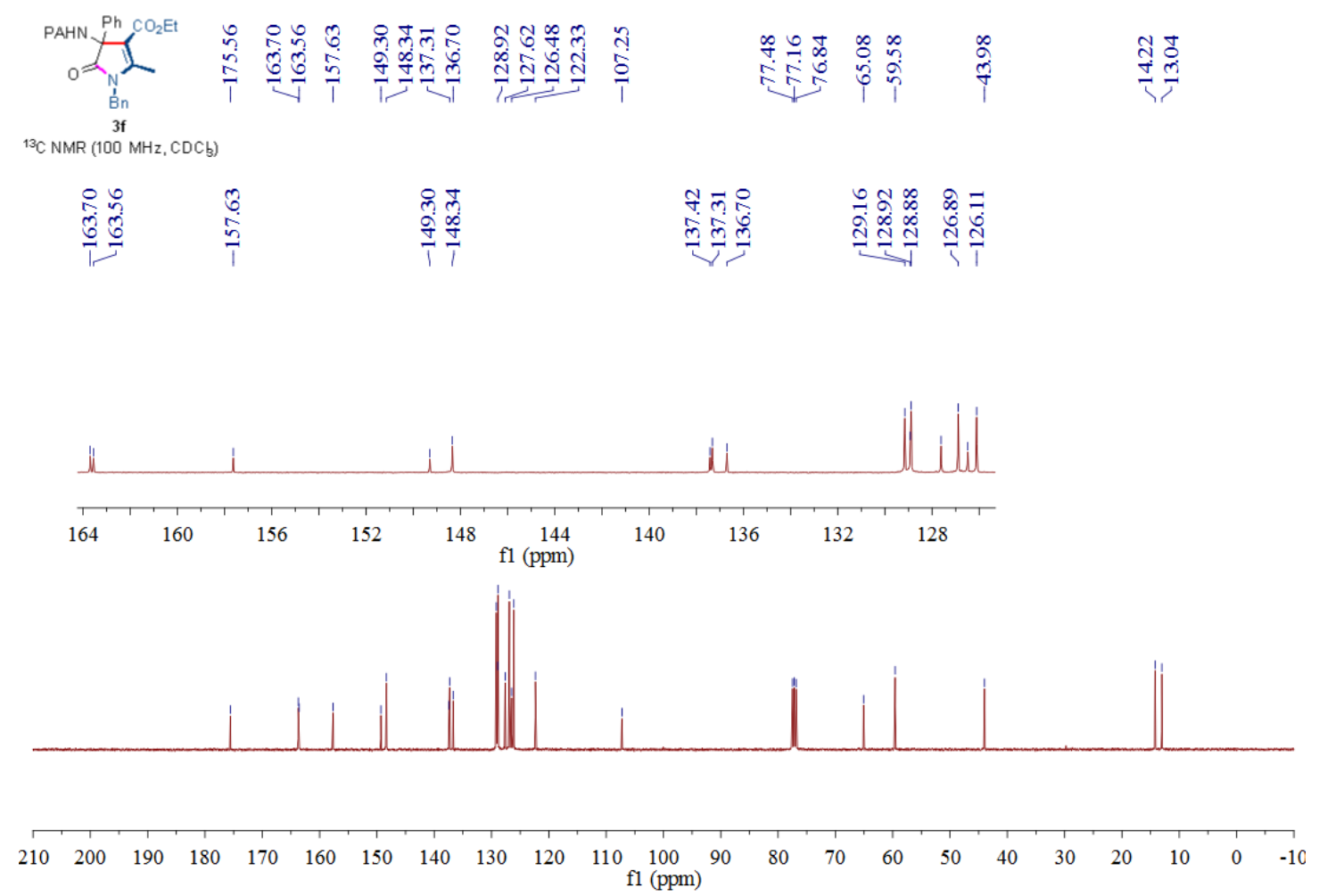

${ }^{1} \mathrm{H}$ NMR and ${ }^{13} \mathrm{C}$ NMR spectra for compound $\mathbf{3 f}$ (using $\mathrm{CDCl}_{3}$ as solvent)

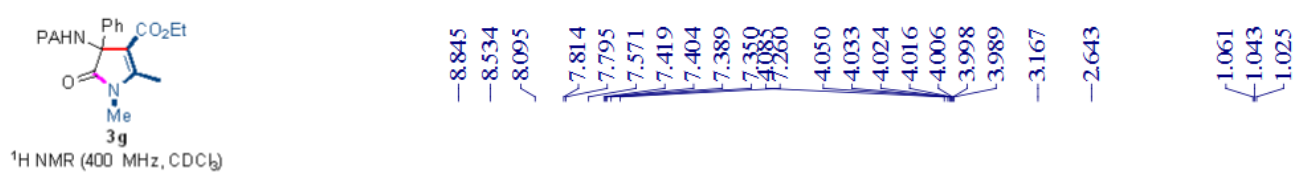

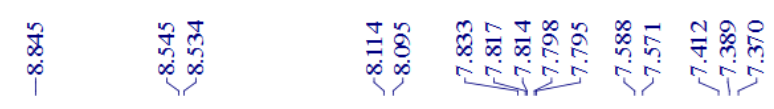
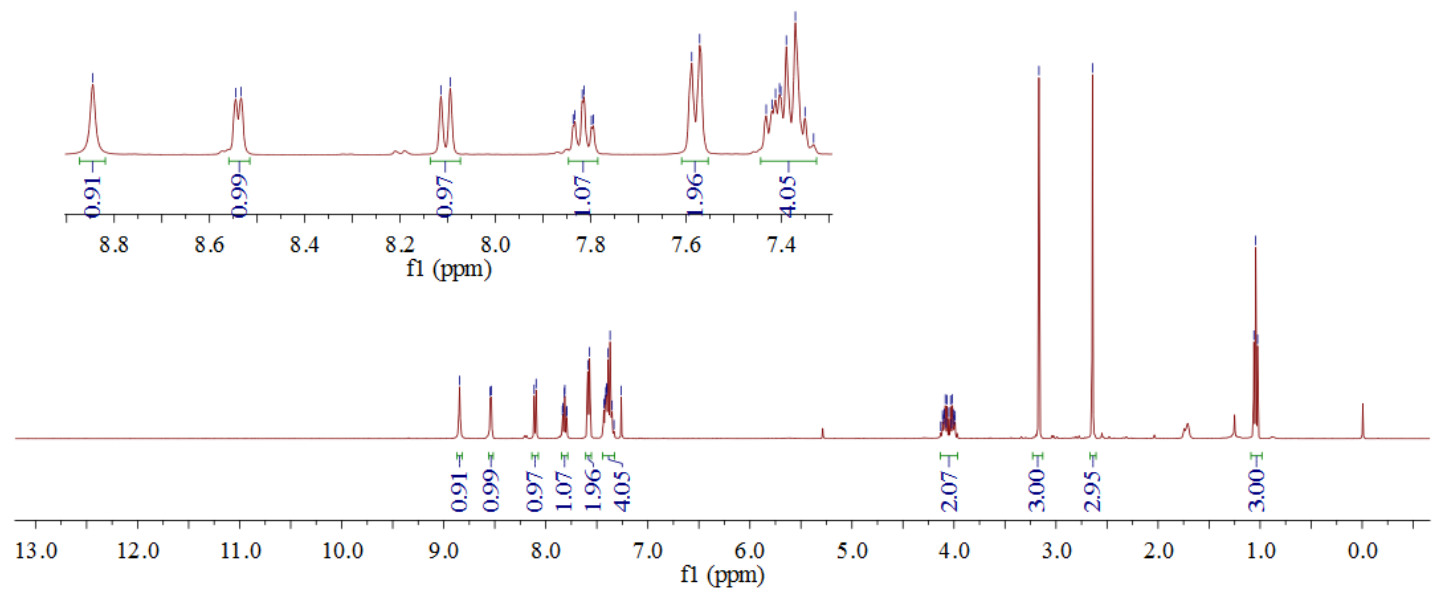


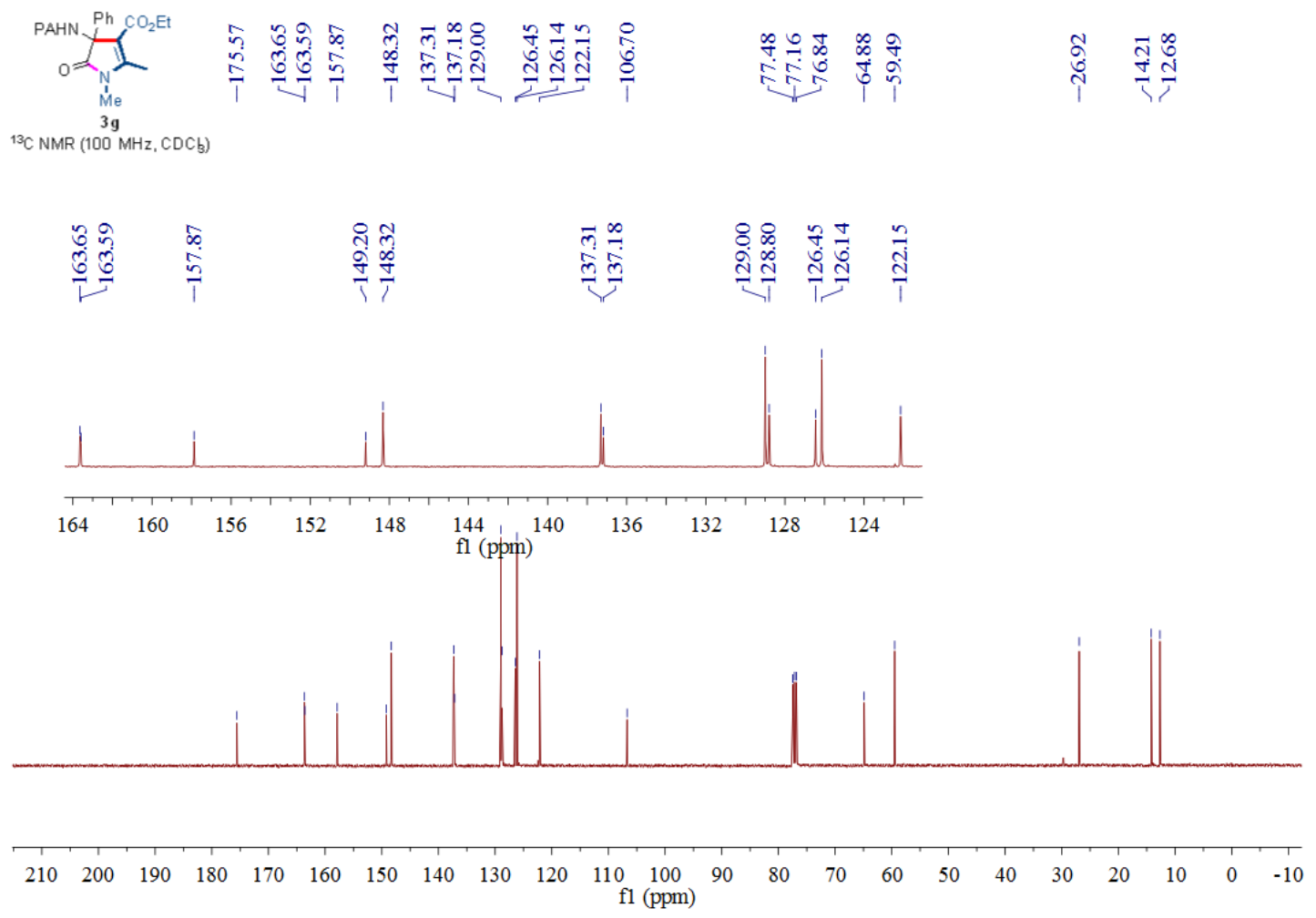

${ }^{1} \mathrm{H}$ NMR and ${ }^{13} \mathrm{C}$ NMR spectra for compound $\mathbf{3 g}$ (using $\mathrm{CDCl}_{3}$ as solvent)

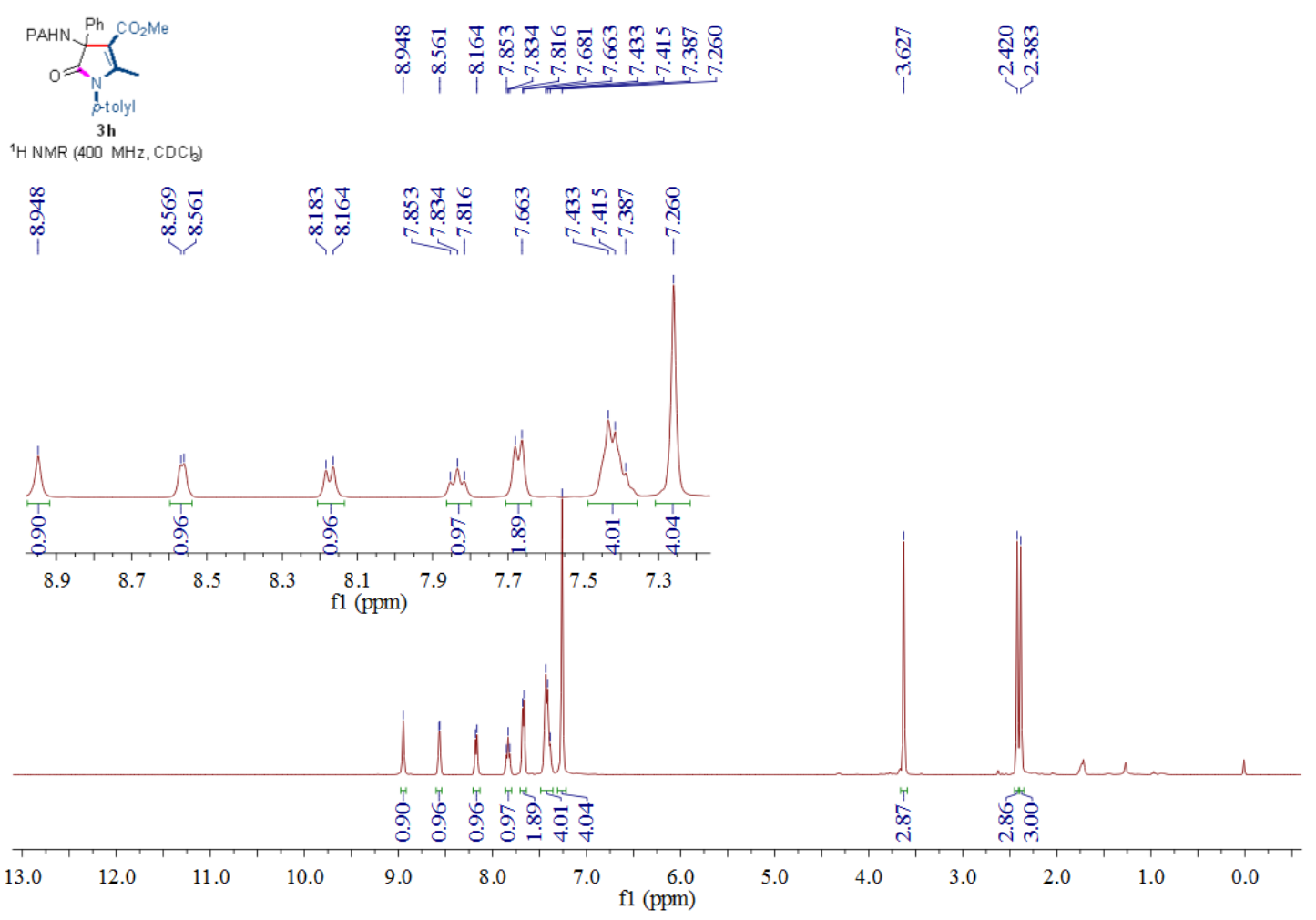




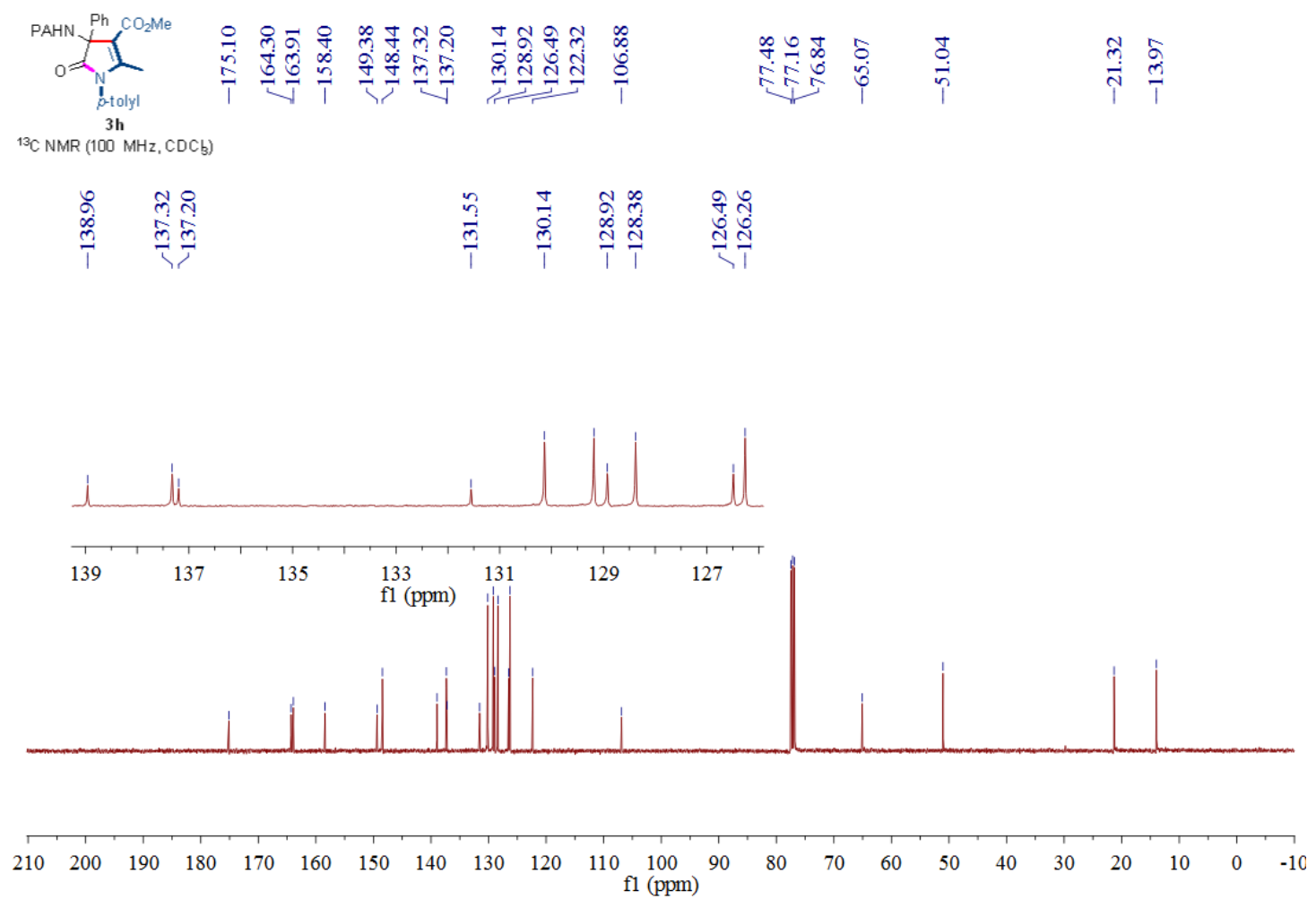

${ }^{1} \mathrm{H}$ NMR and ${ }^{13} \mathrm{C}$ NMR spectra for compound $\mathbf{3 h}$ (using $\mathrm{CDCl}_{3}$ as solvent)
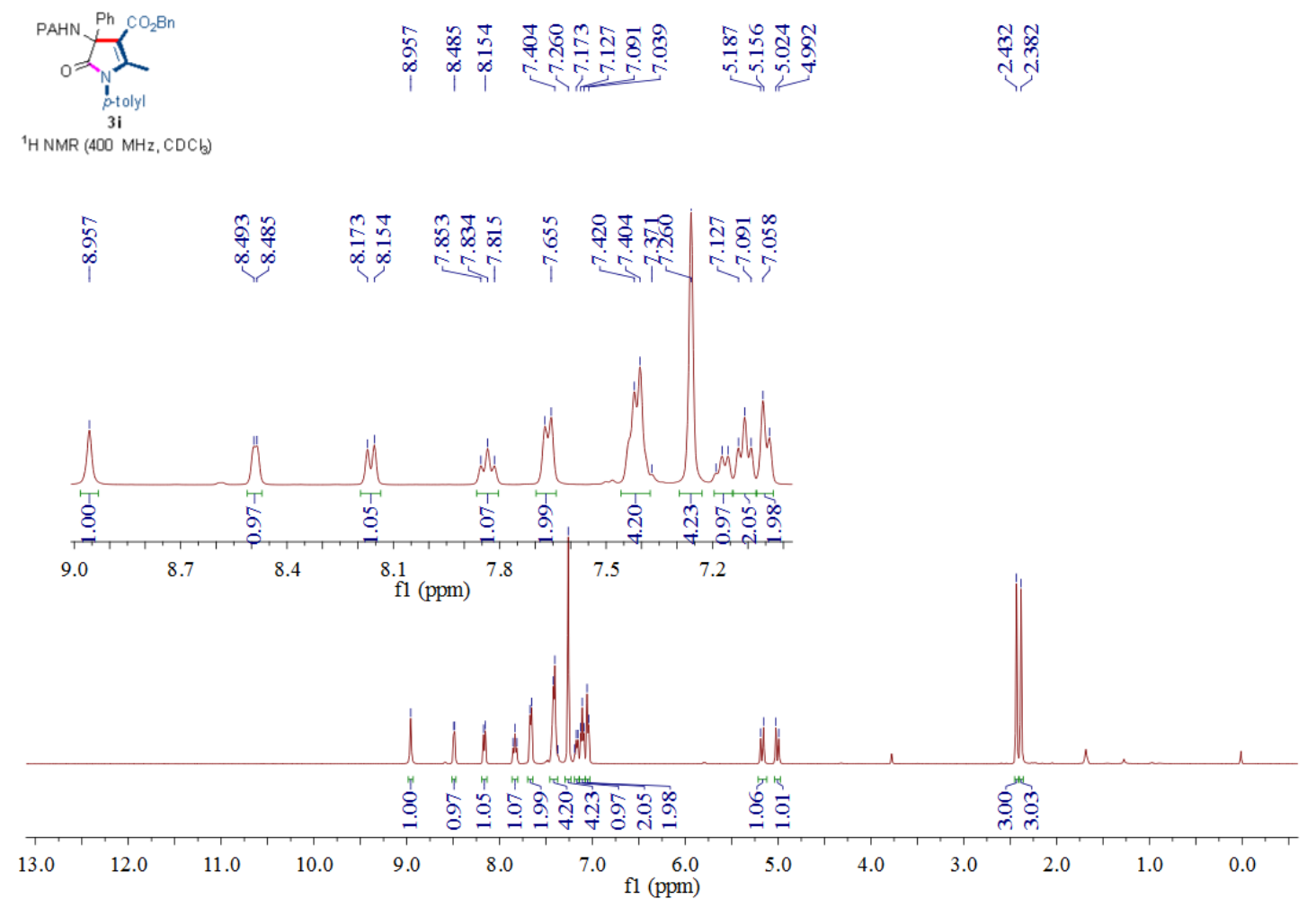


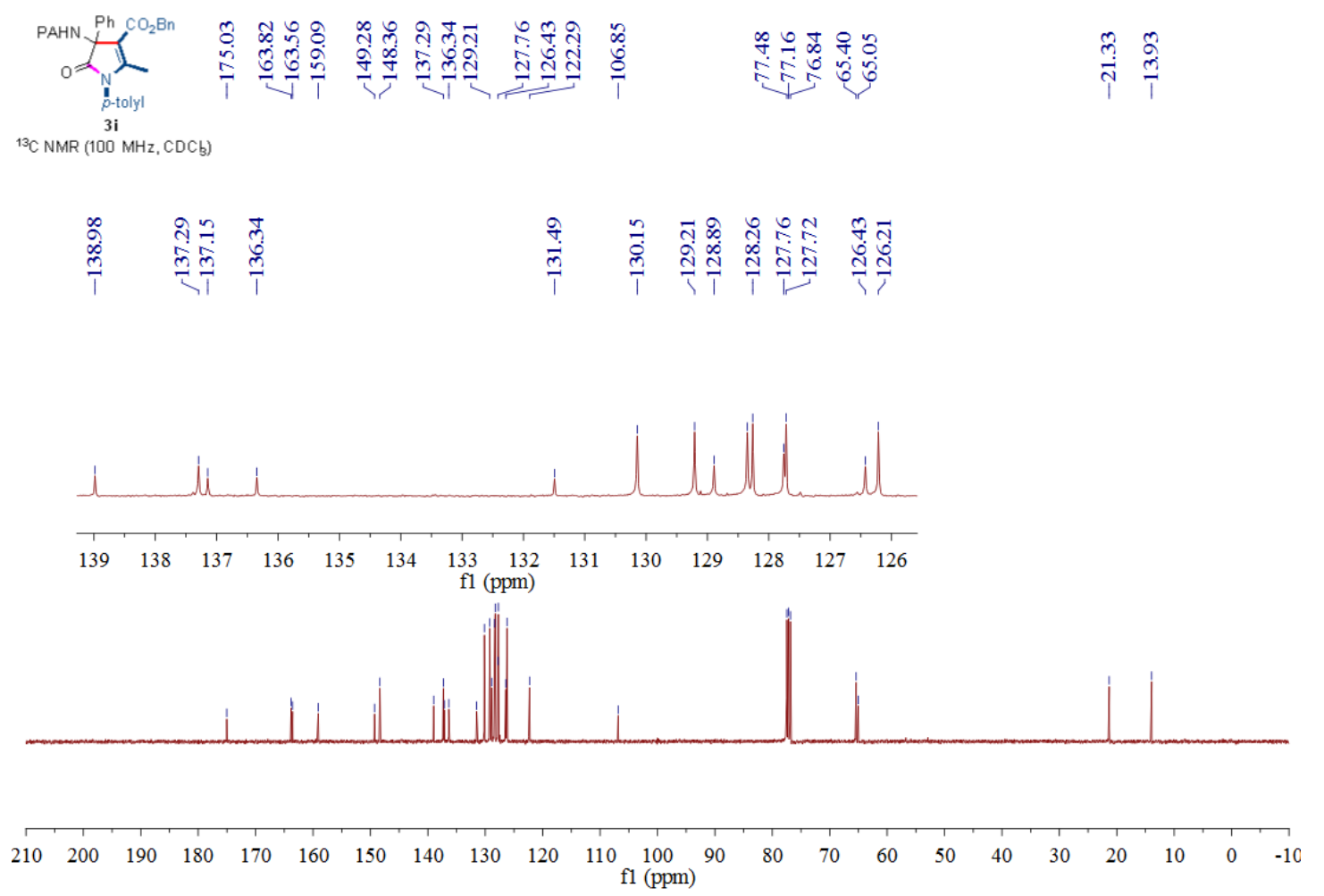

${ }^{1} \mathrm{H}$ NMR and ${ }^{13} \mathrm{C}$ NMR spectra for compound $3 \mathbf{i}$ (using $\mathrm{CDCl}_{3}$ as solvent)

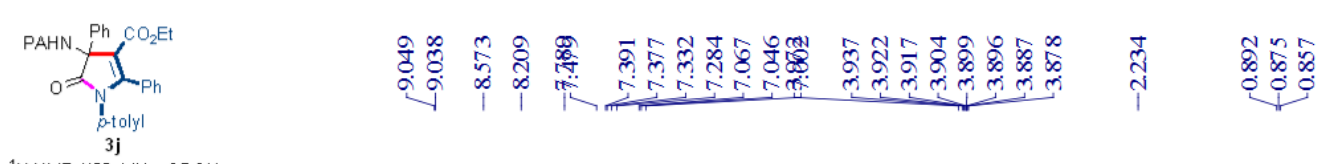

${ }^{1} \mathrm{H} N M R(400 \mathrm{MHz}, \mathrm{CDC} 6)$

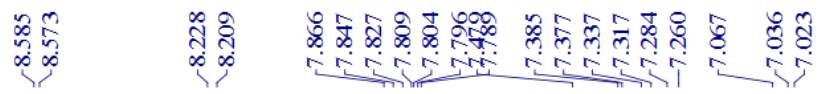
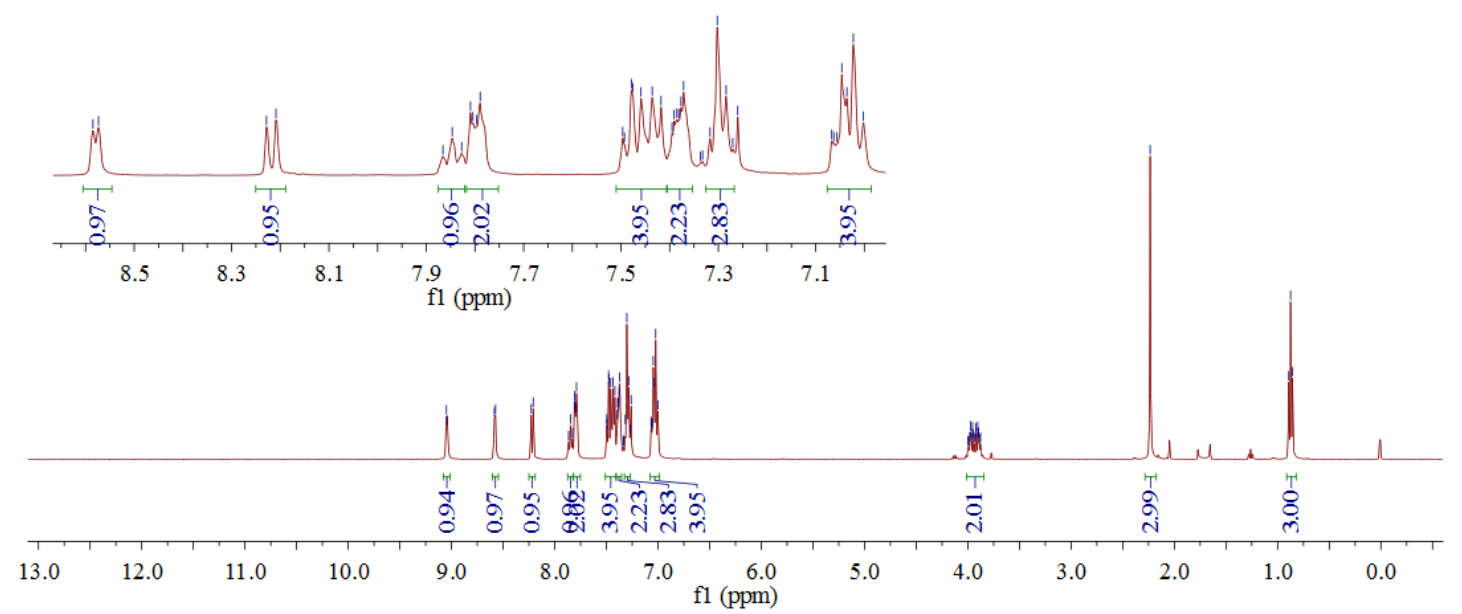

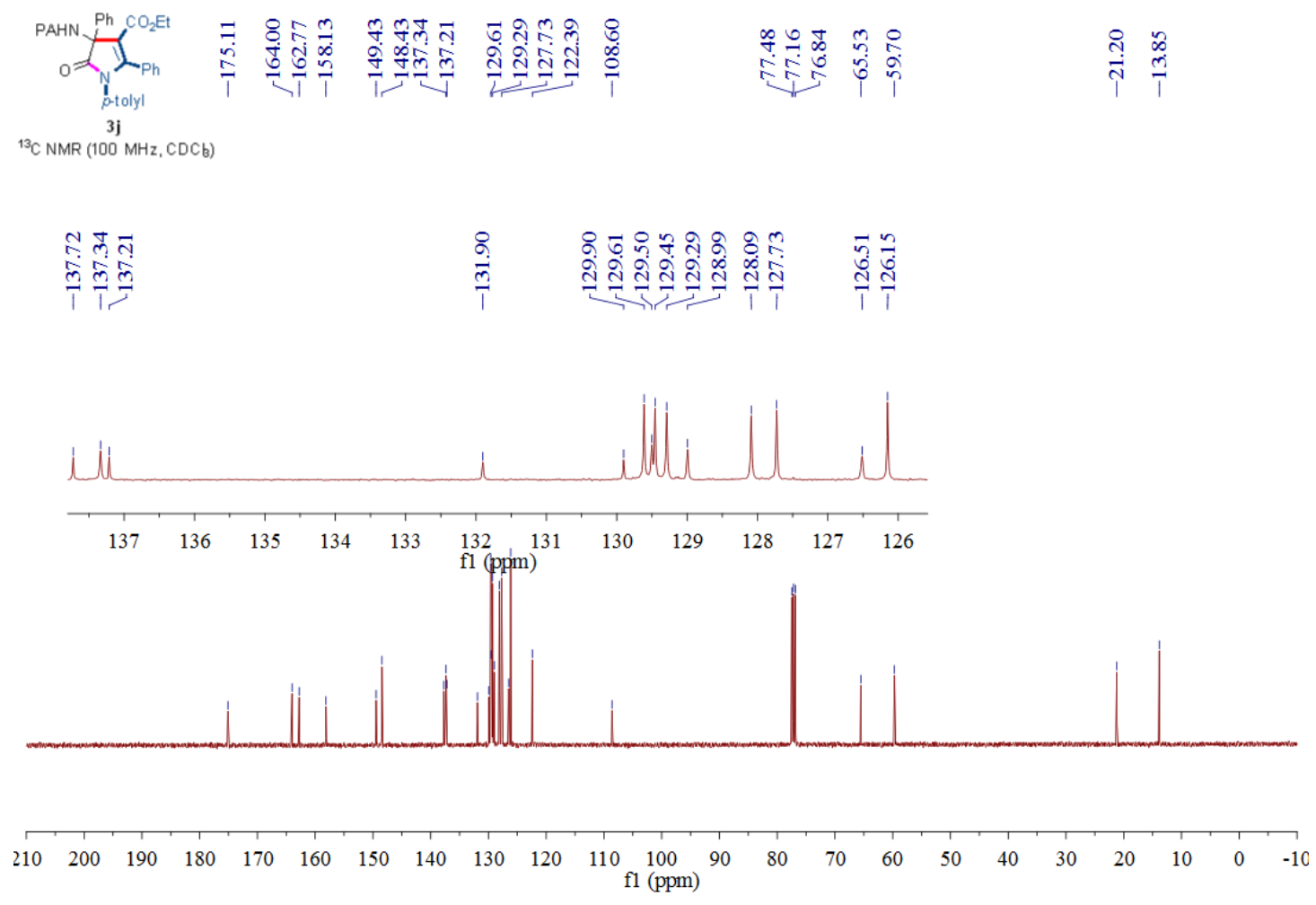

${ }^{1} \mathrm{H}$ NMR and ${ }^{13} \mathrm{C}$ NMR spectra for compound $\mathbf{3 j}$ (using $\mathrm{CDCl}_{3}$ as solvent)

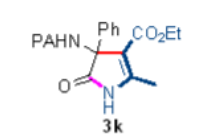

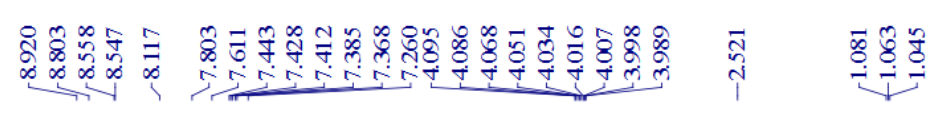

${ }^{1} \mathrm{H}$ NMR $(400 \mathrm{MHz}, \mathrm{CDCb})$

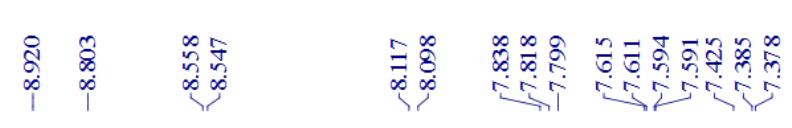
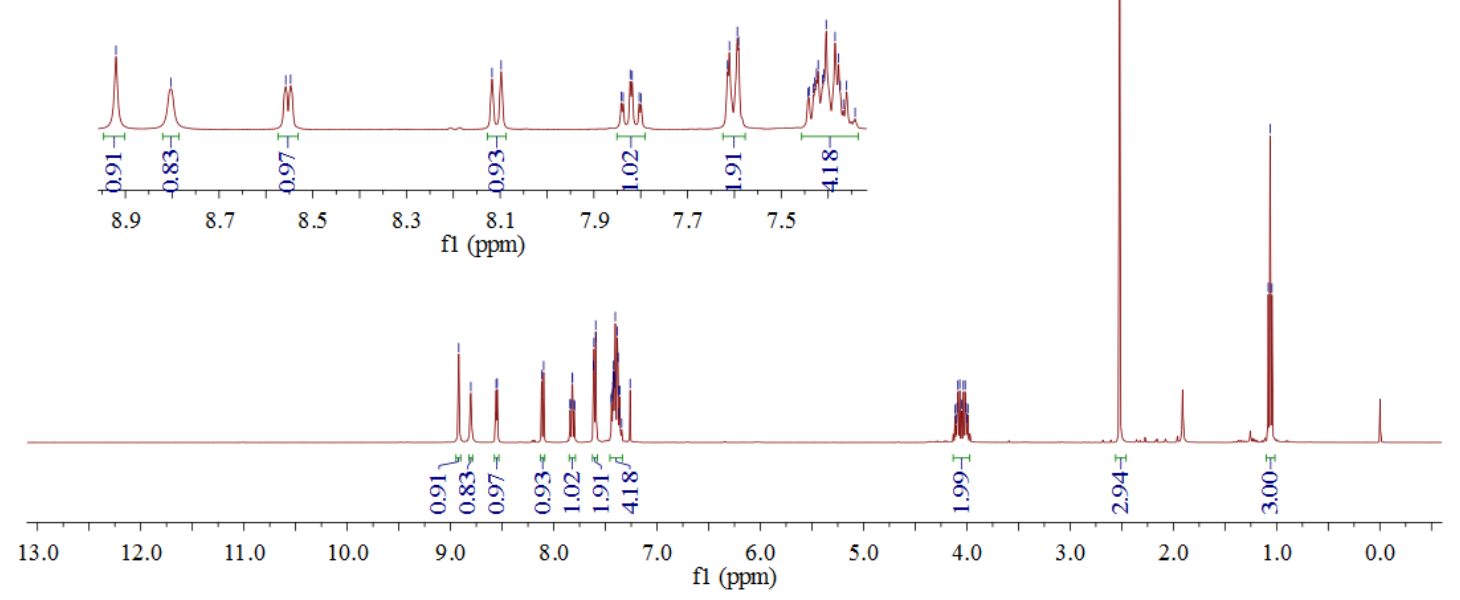


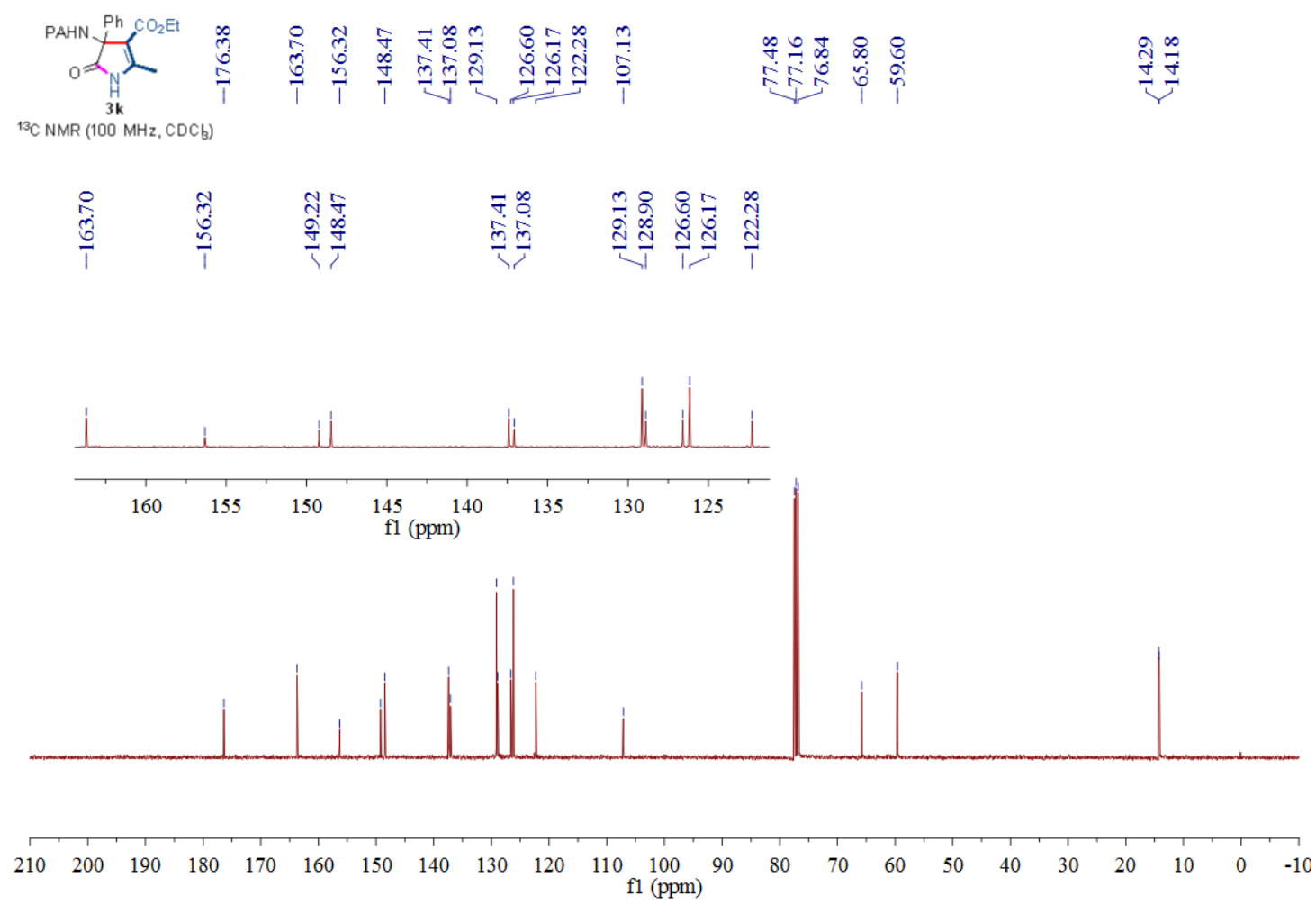

${ }^{1} \mathrm{H}$ NMR and ${ }^{13} \mathrm{C}$ NMR spectra for compound $\mathbf{3 k}$ (using $\mathrm{CDCl}_{3}$ as solvent)

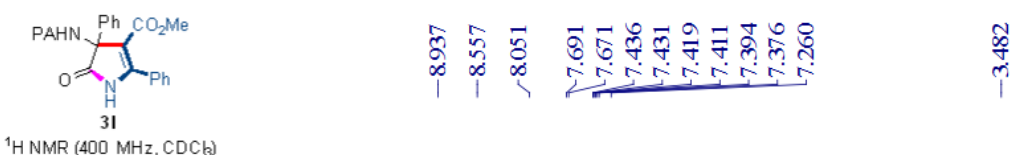

${ }^{1} \mathrm{H}$ NMR $(400 \mathrm{MHz}, \mathrm{CDCb})$

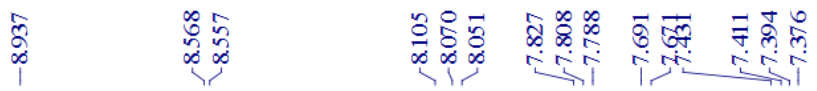
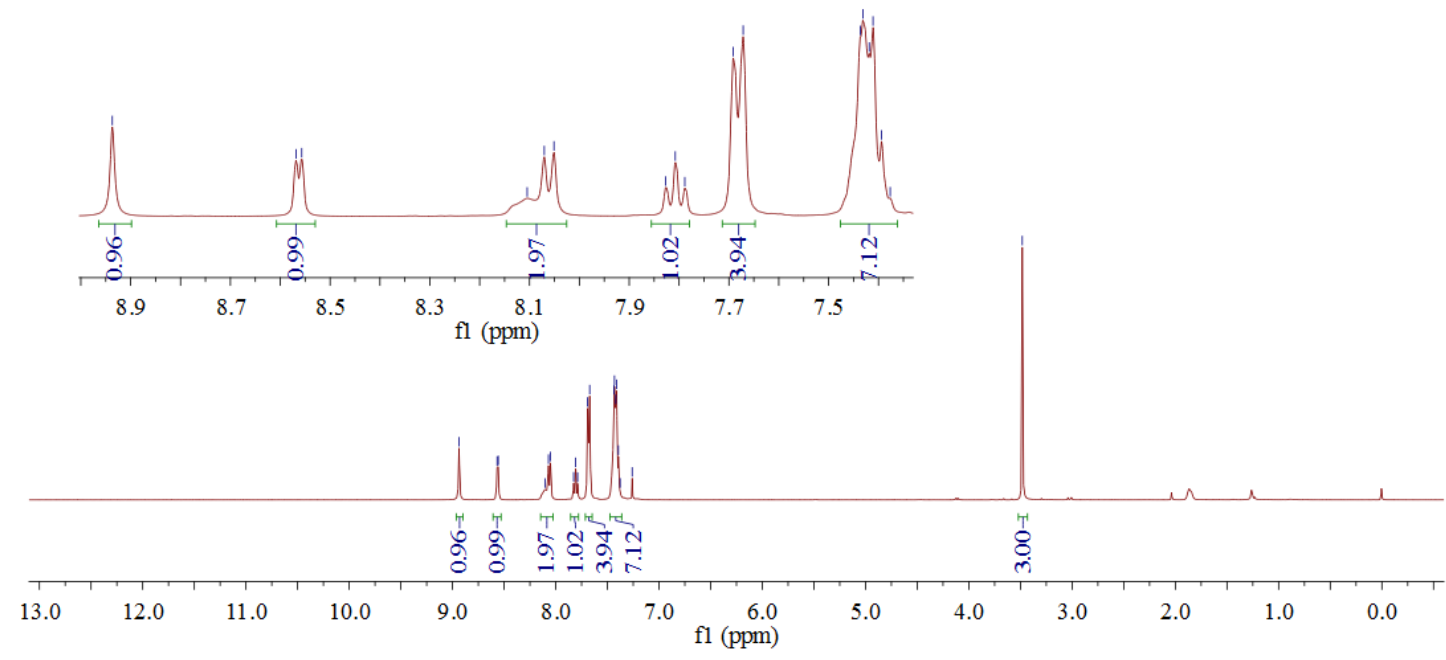


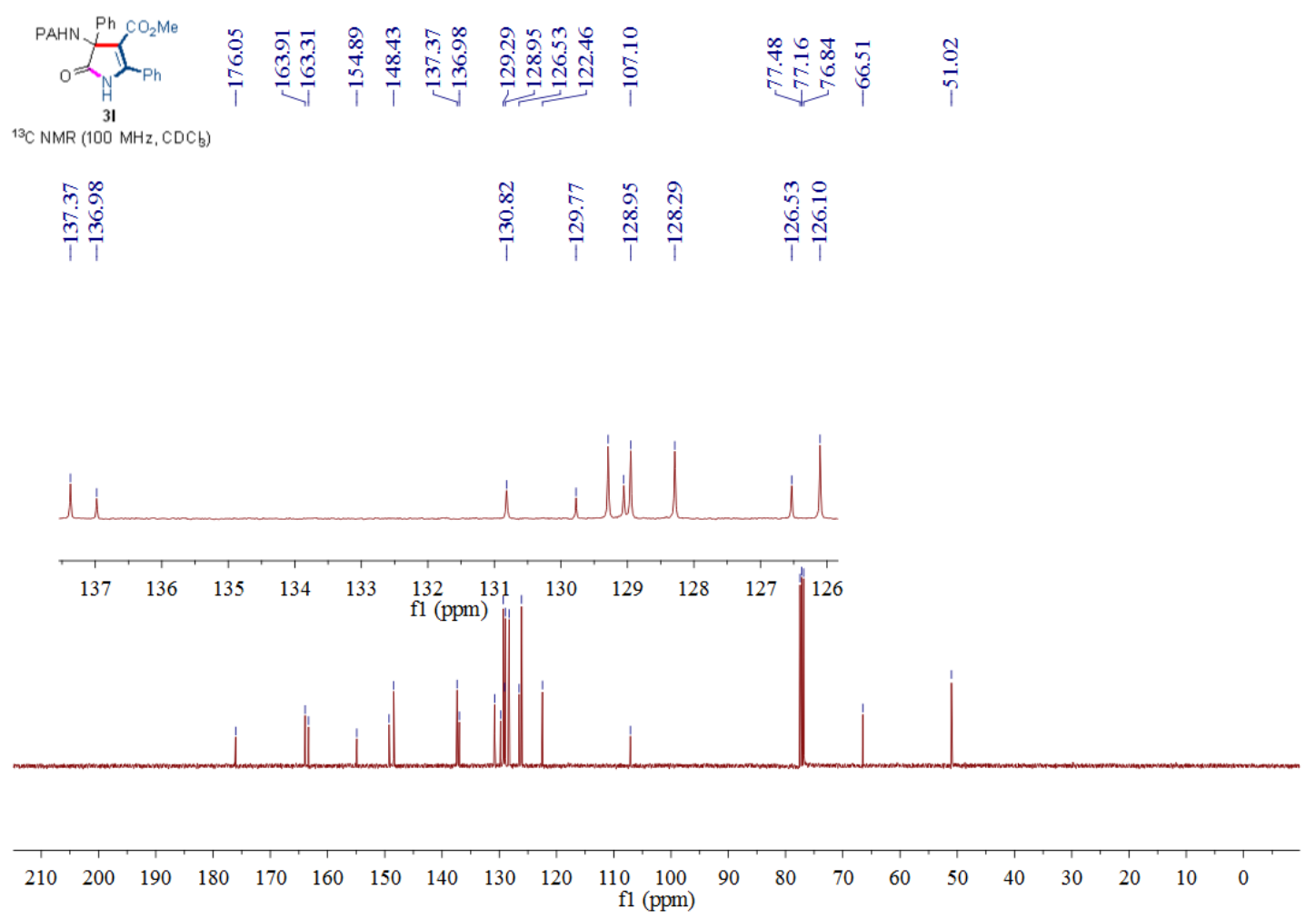

${ }^{1} \mathrm{H}$ NMR and ${ }^{13} \mathrm{C}$ NMR spectra for compound 31 (using $\mathrm{CDCl}_{3}$ as solvent)

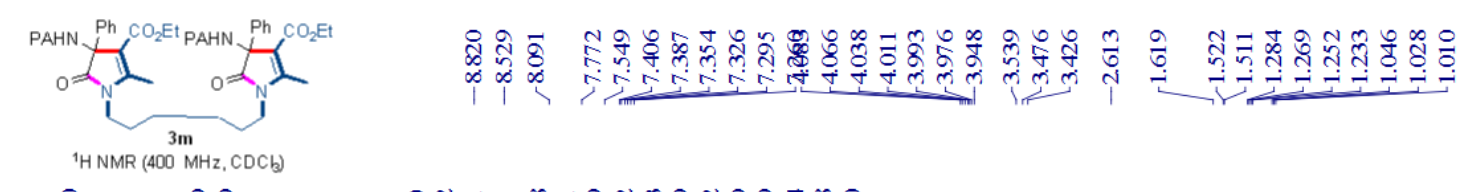

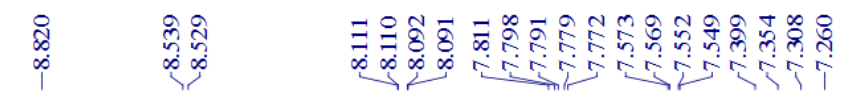

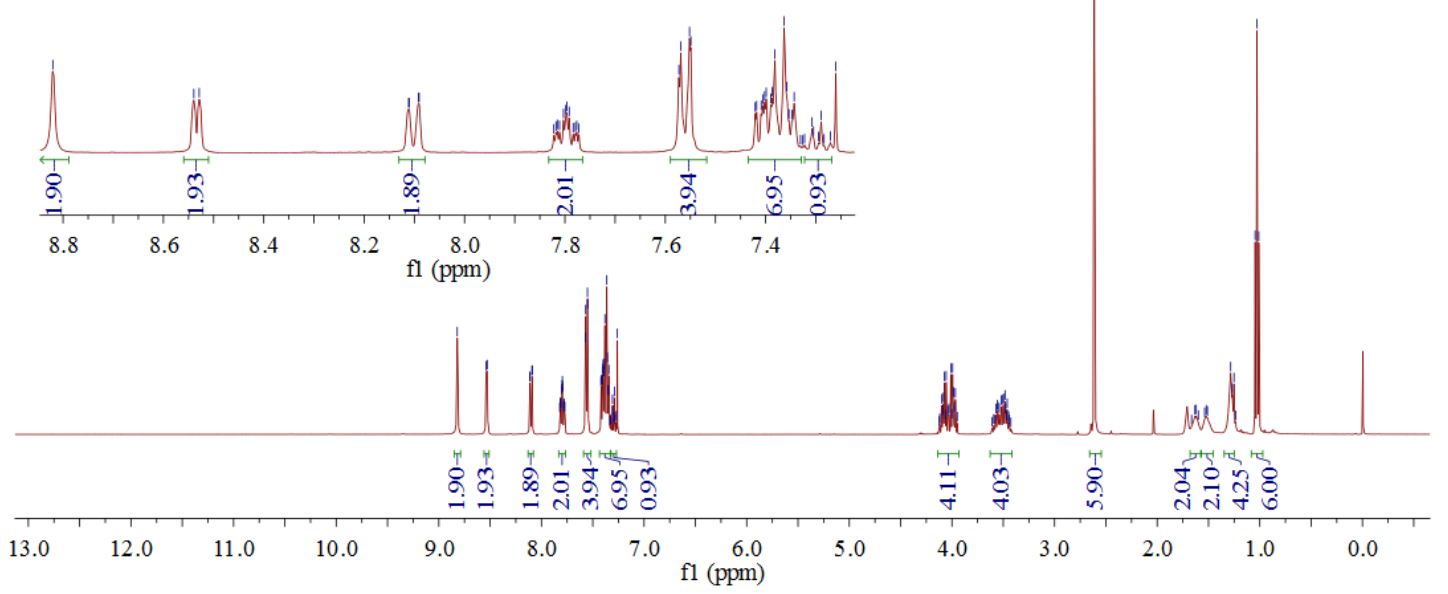




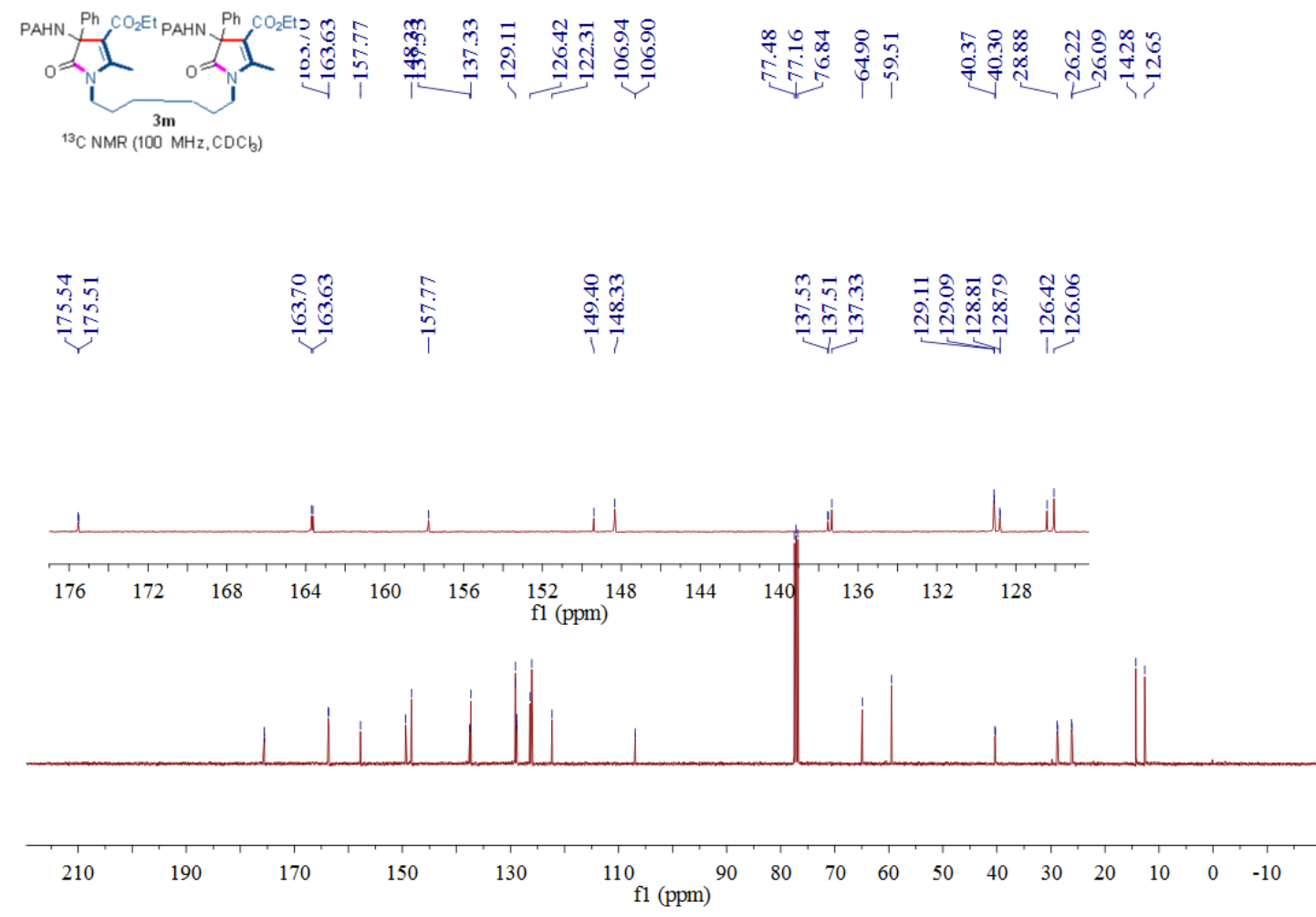

${ }^{1} \mathrm{H}$ NMR and ${ }^{13} \mathrm{C}$ NMR spectra for compound $\mathbf{3 m}$ (using $\mathrm{CDCl}_{3}$ as solvent)

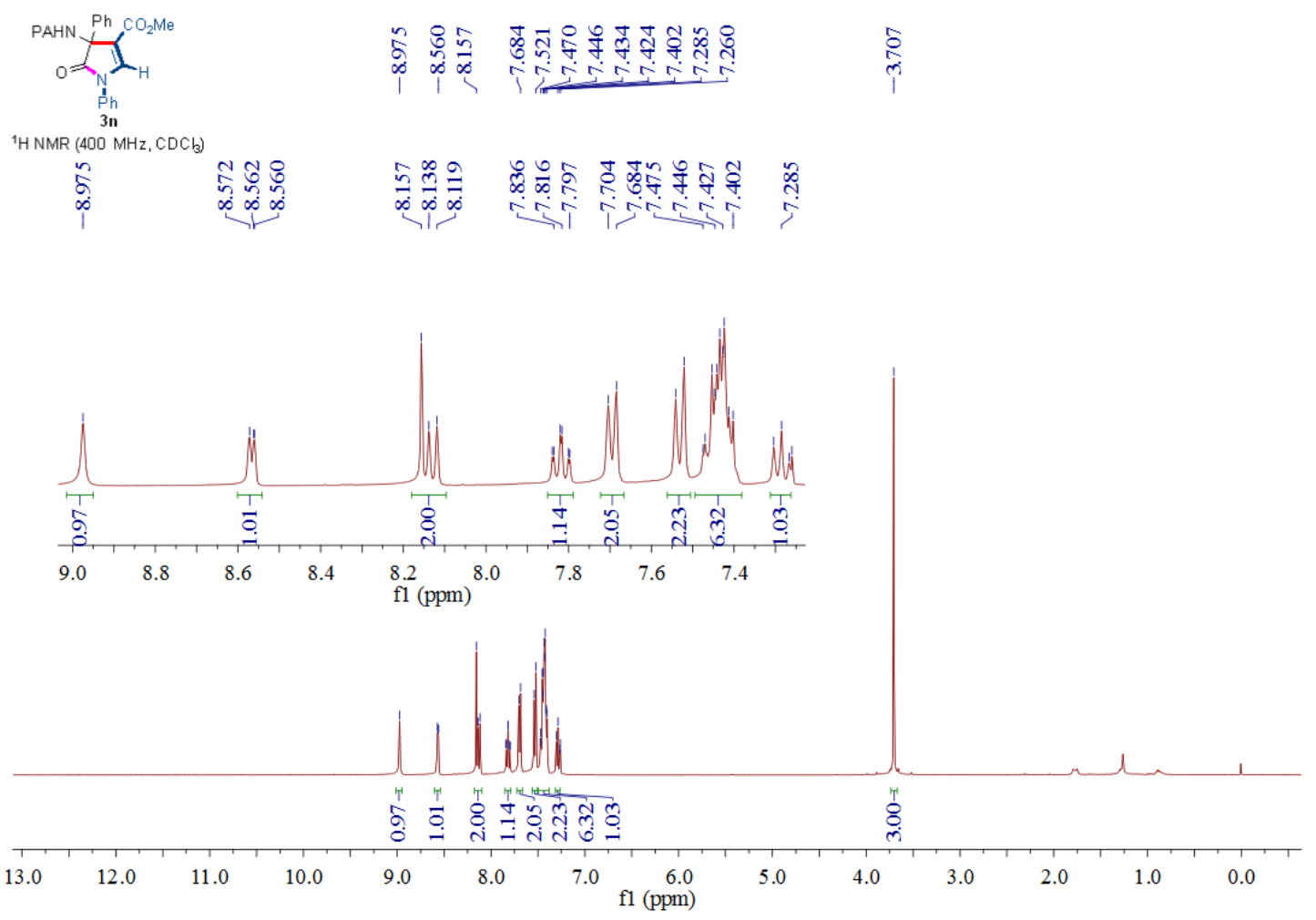




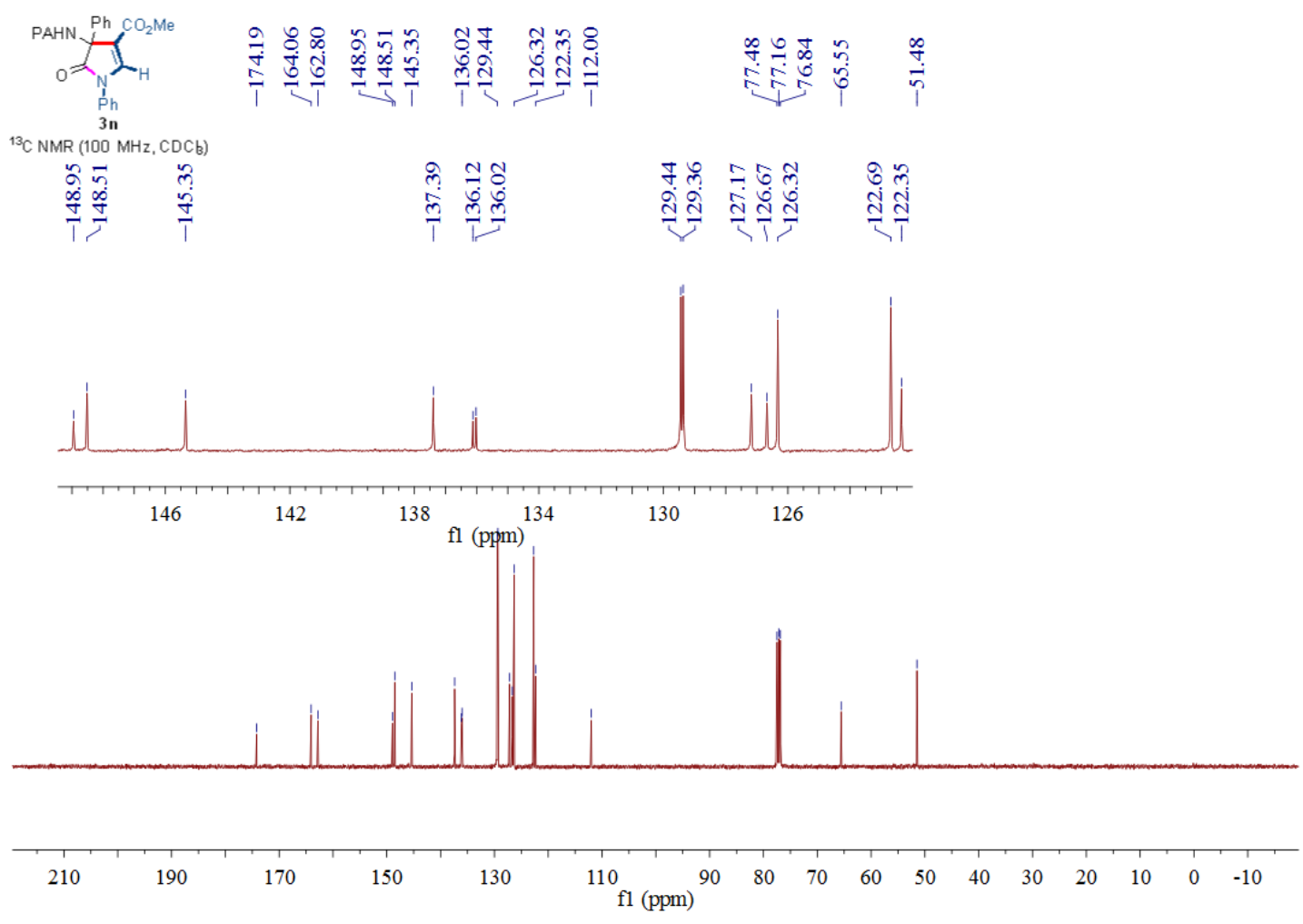

${ }^{1} \mathrm{H}$ NMR and ${ }^{13} \mathrm{C}$ NMR spectra for compound 3 n (using $\mathrm{CDCl}_{3}$ as solvent)

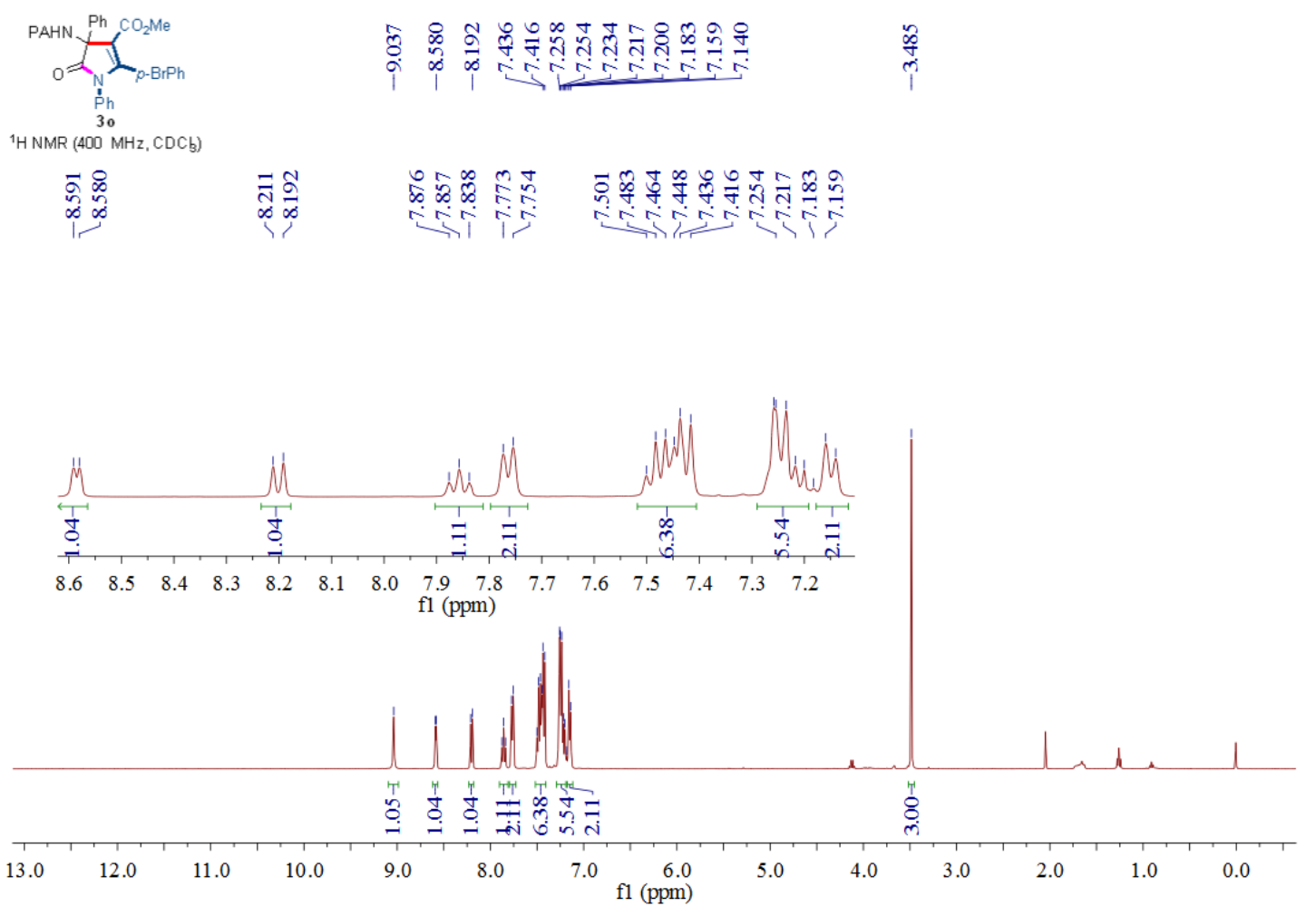



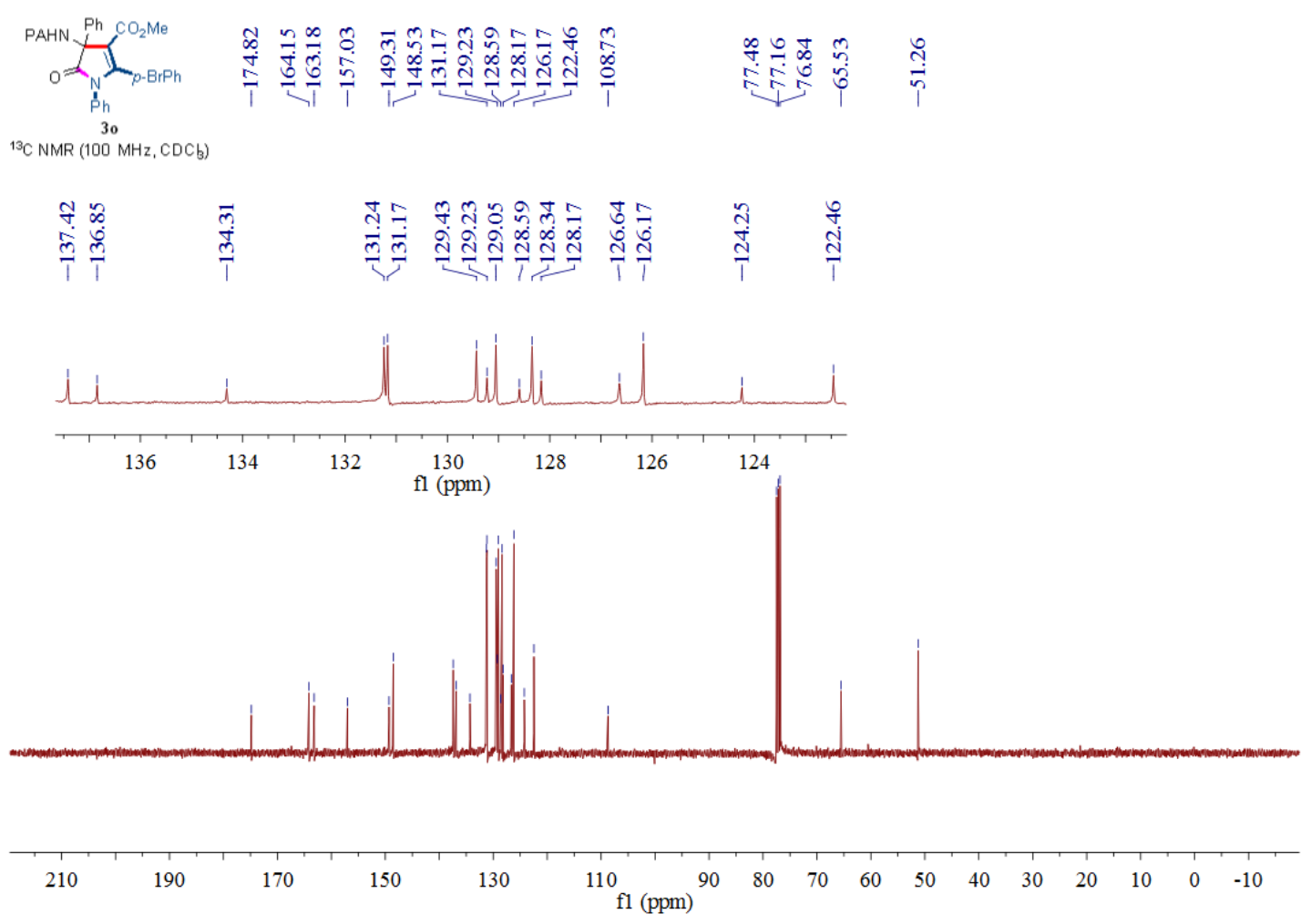

${ }^{1} \mathrm{H}$ NMR and ${ }^{13} \mathrm{C}$ NMR spectra for compound 30 (using $\mathrm{CDCl}_{3}$ as solvent)

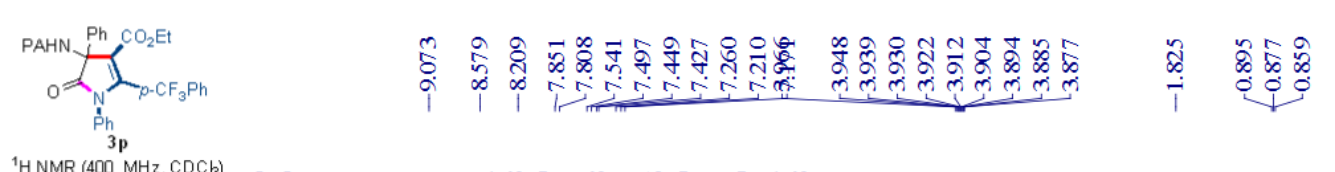

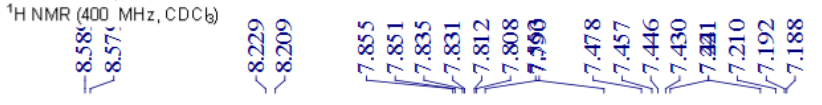
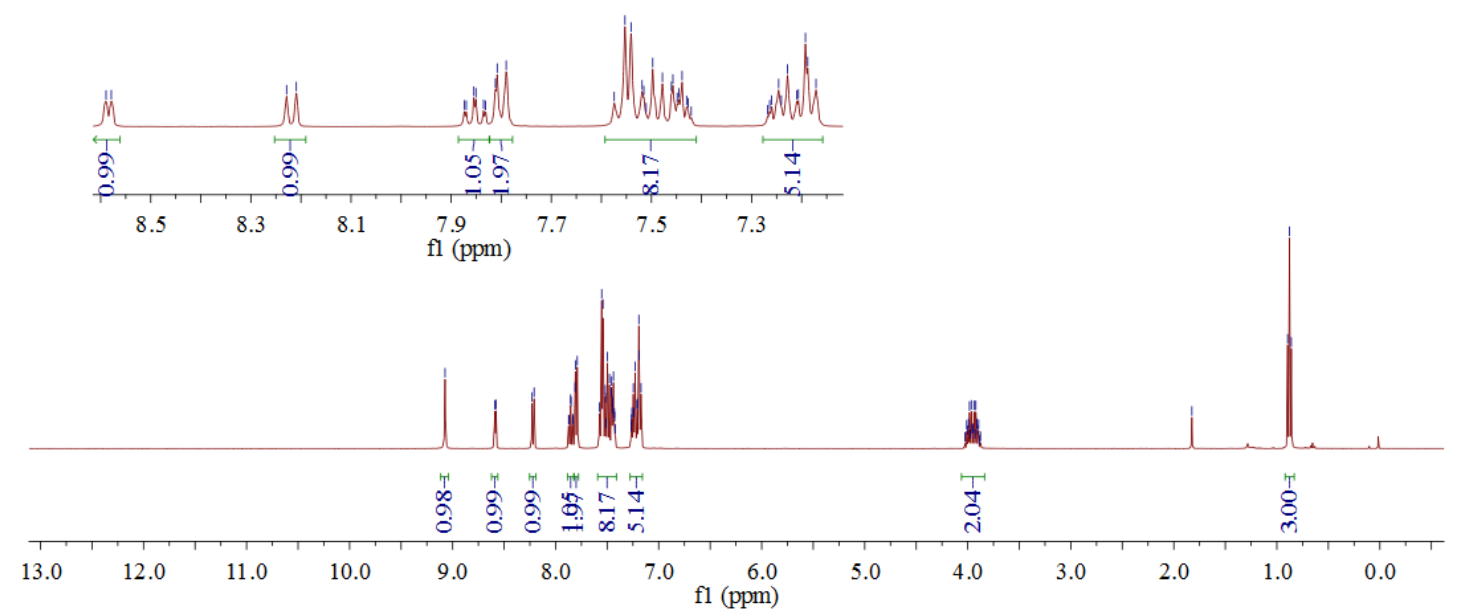


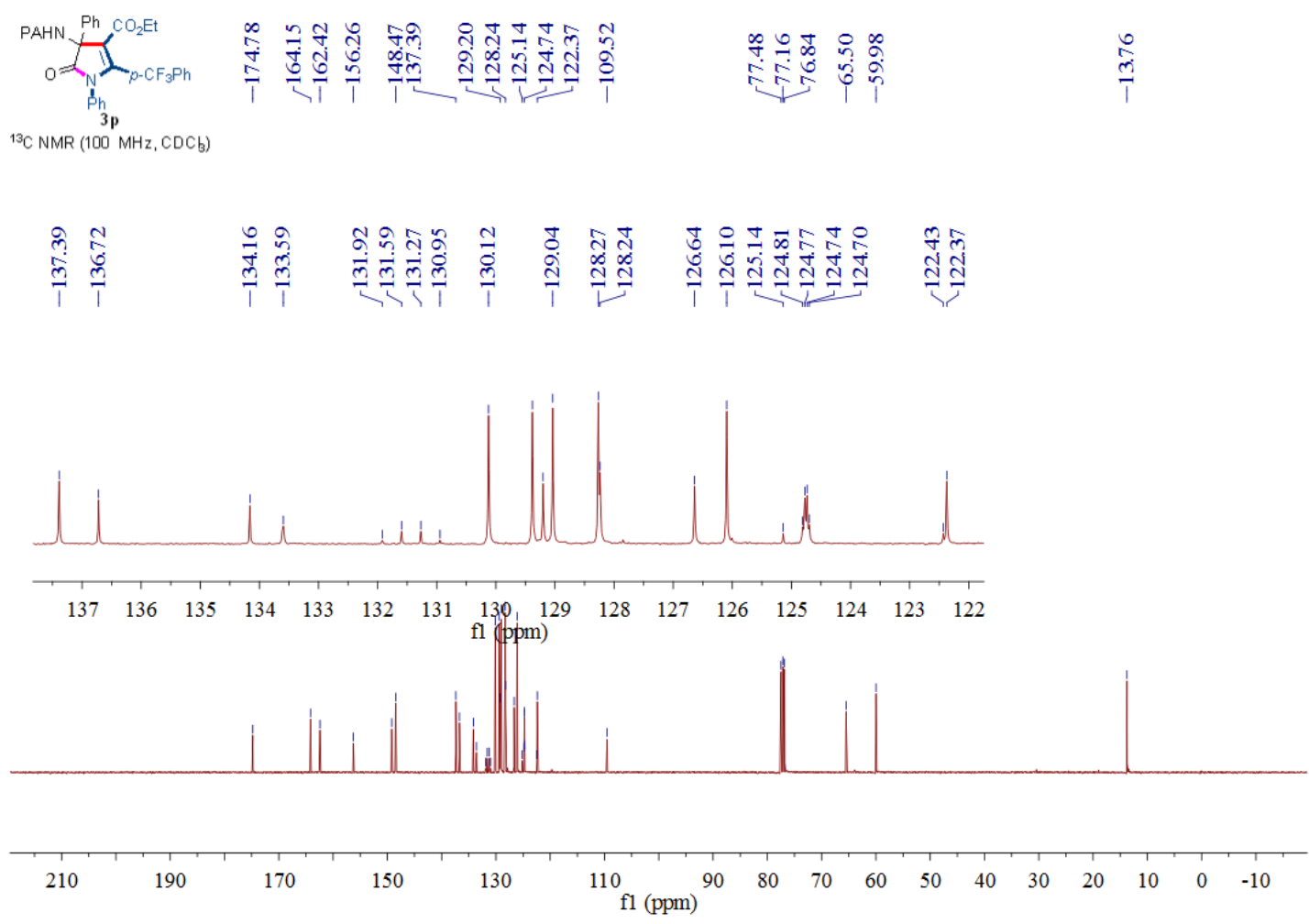

${ }^{1} \mathrm{H}$ NMR and ${ }^{13} \mathrm{C}$ NMR spectra for compound $\mathbf{3 p}$ (using $\mathrm{CDCl}_{3}$ as solvent)

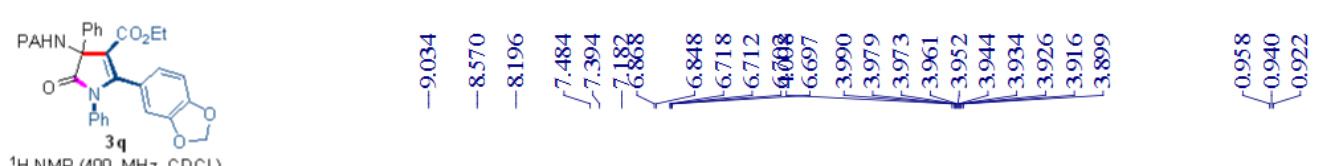

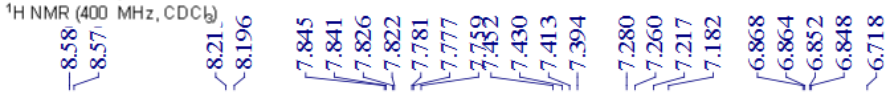

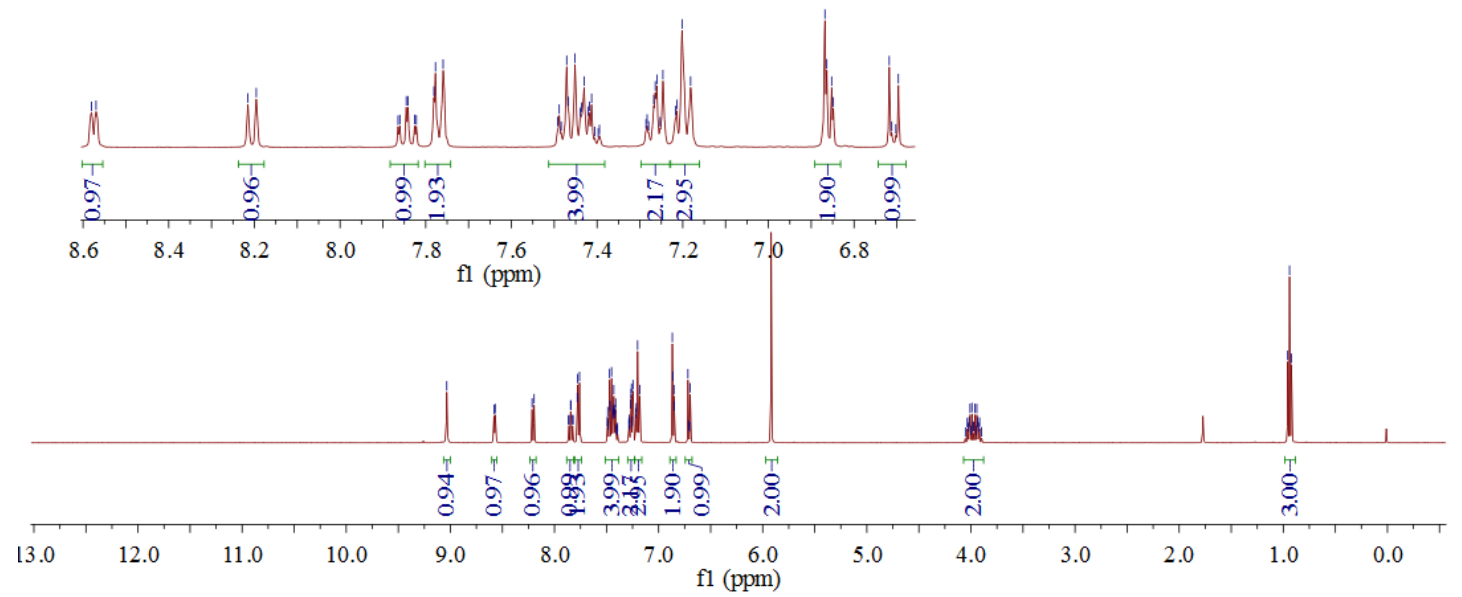



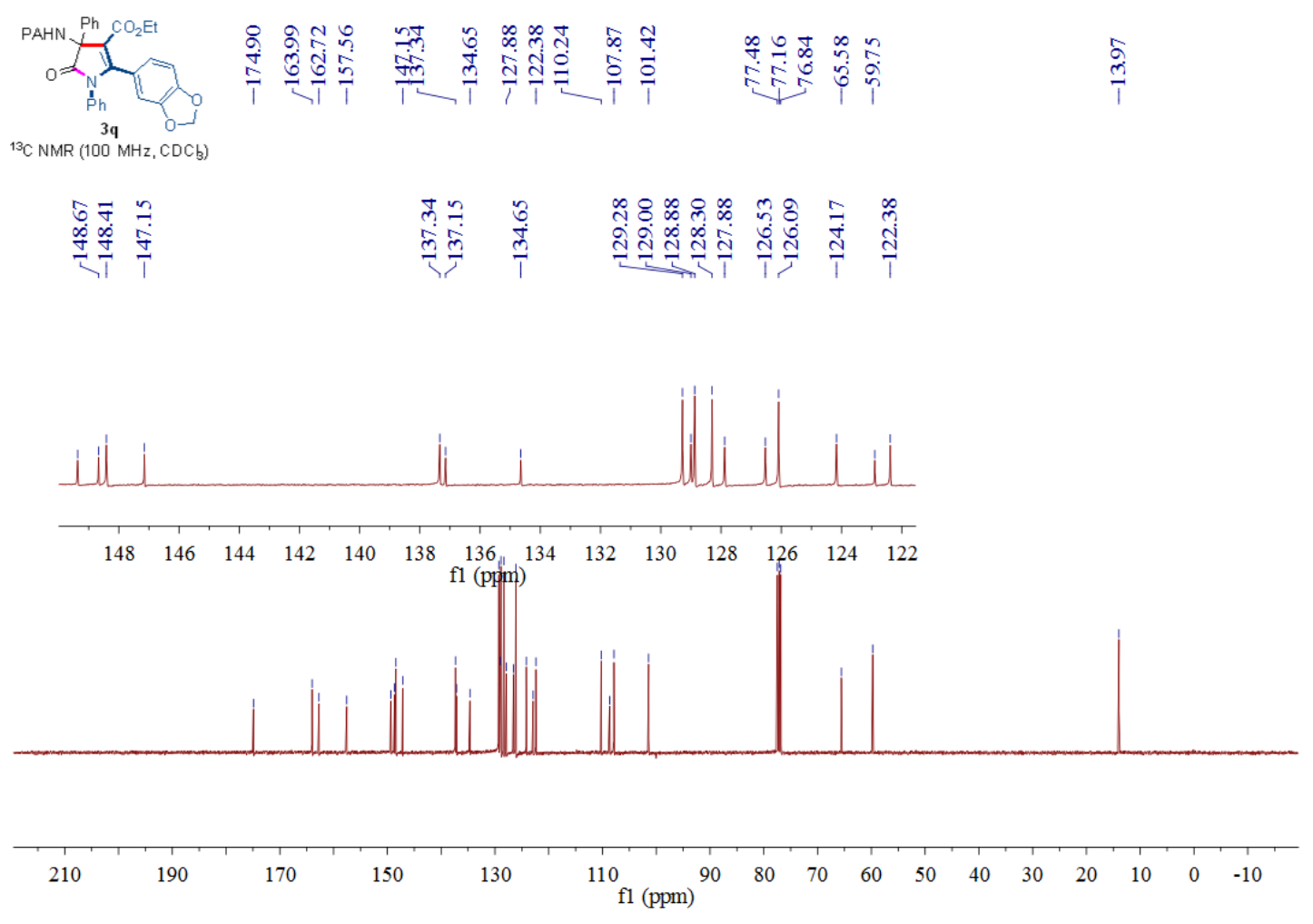

${ }^{1} \mathrm{H}$ NMR and ${ }^{13} \mathrm{C}$ NMR spectra for compound $\mathbf{3 q}$ (using $\mathrm{CDCl}_{3}$ as solvent)

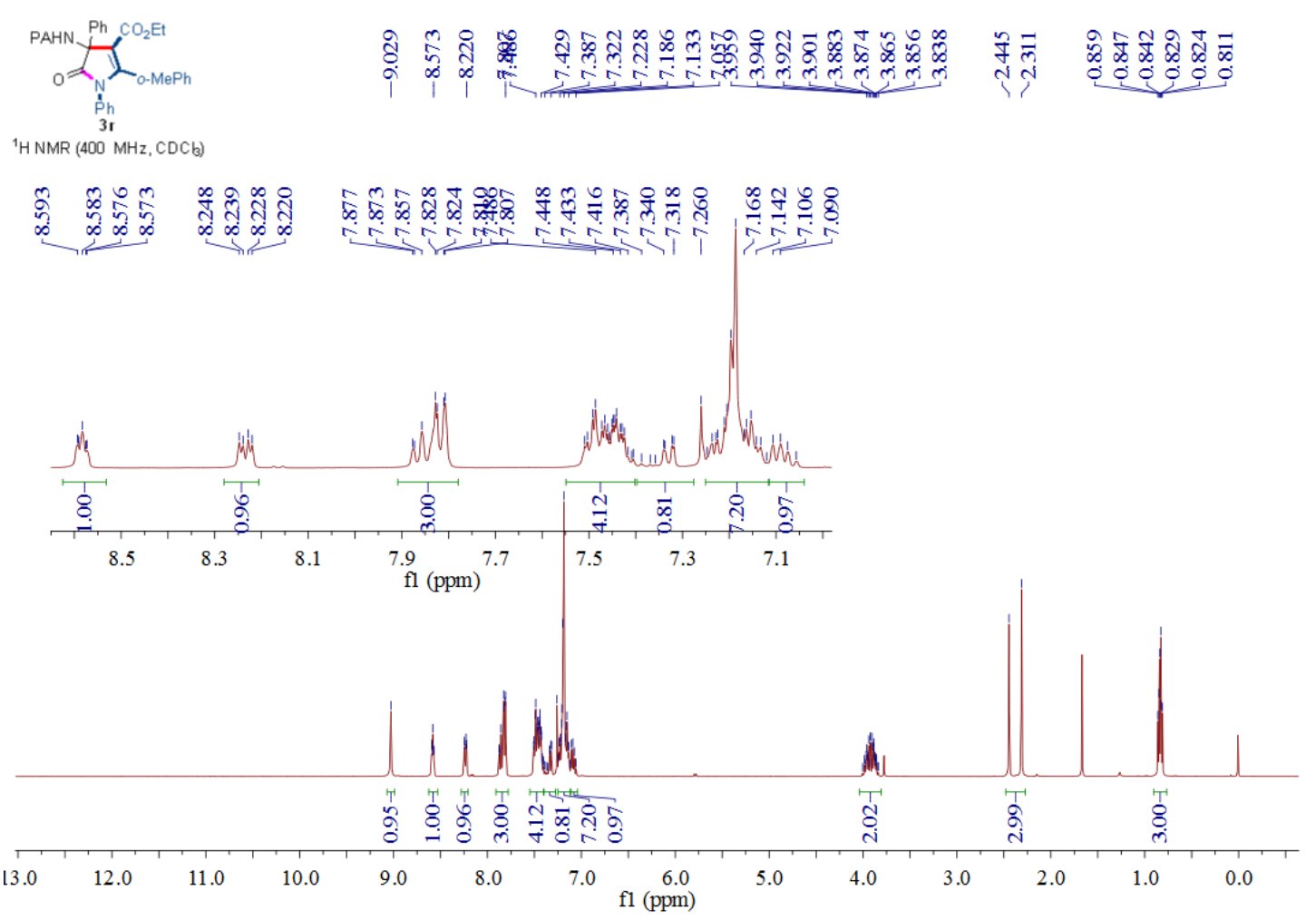



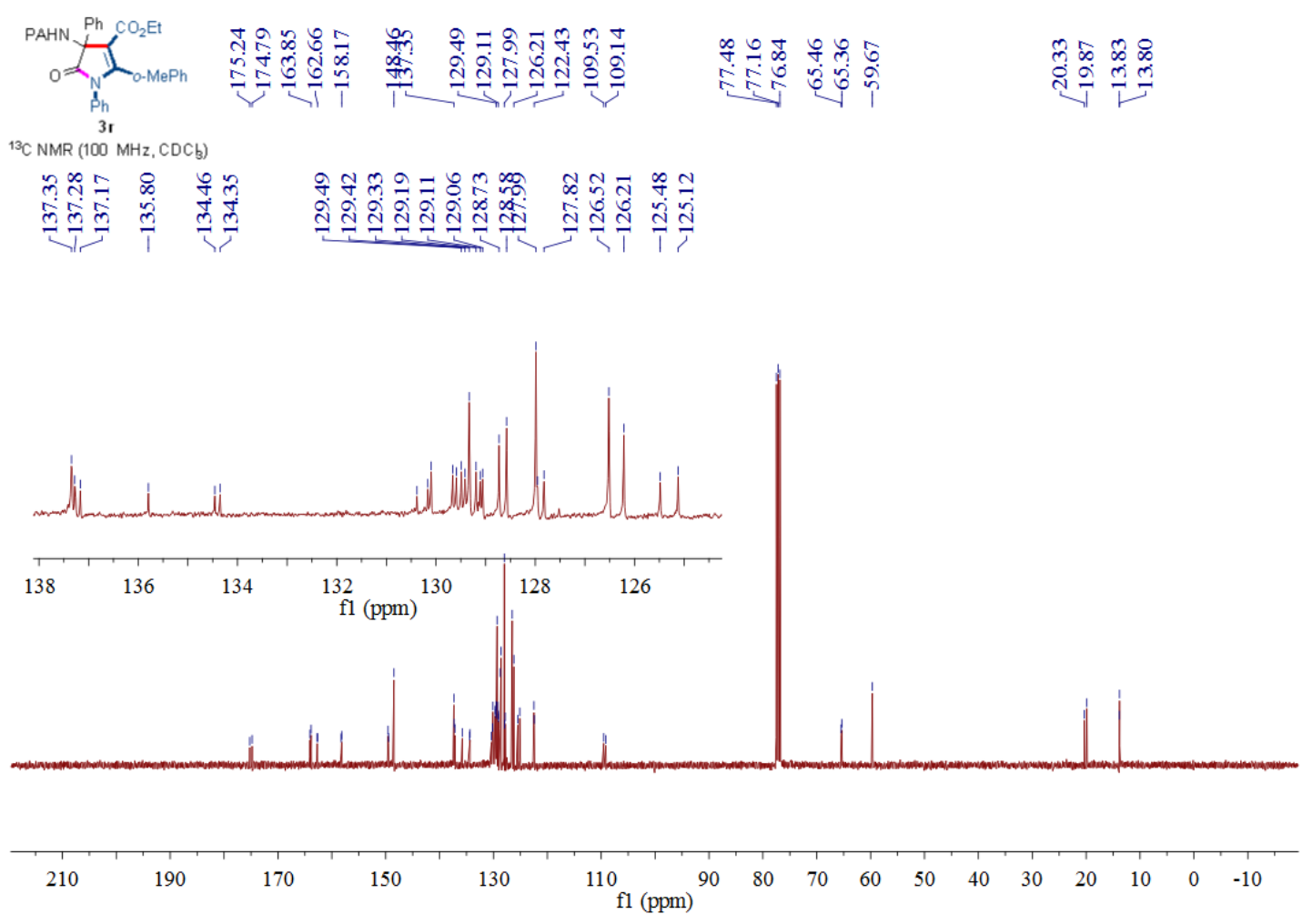

${ }^{1} \mathrm{H}$ NMR and ${ }^{13} \mathrm{C}$ NMR spectra for compound $3 \mathbf{r}$ (using $\mathrm{CDCl}_{3}$ as solvent)

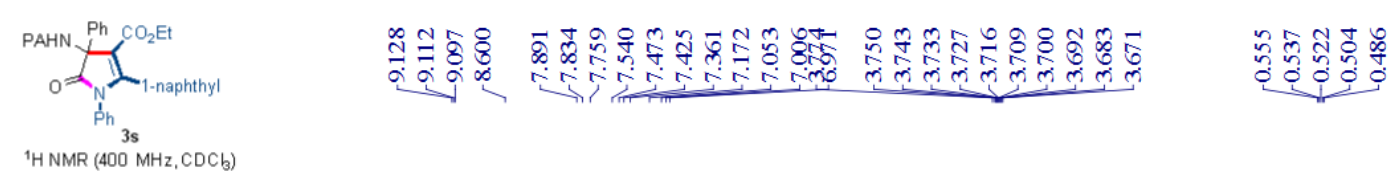

${ }^{1} \mathrm{H}$ NMR $(400 \mathrm{MHz}, \mathrm{CDCl})$
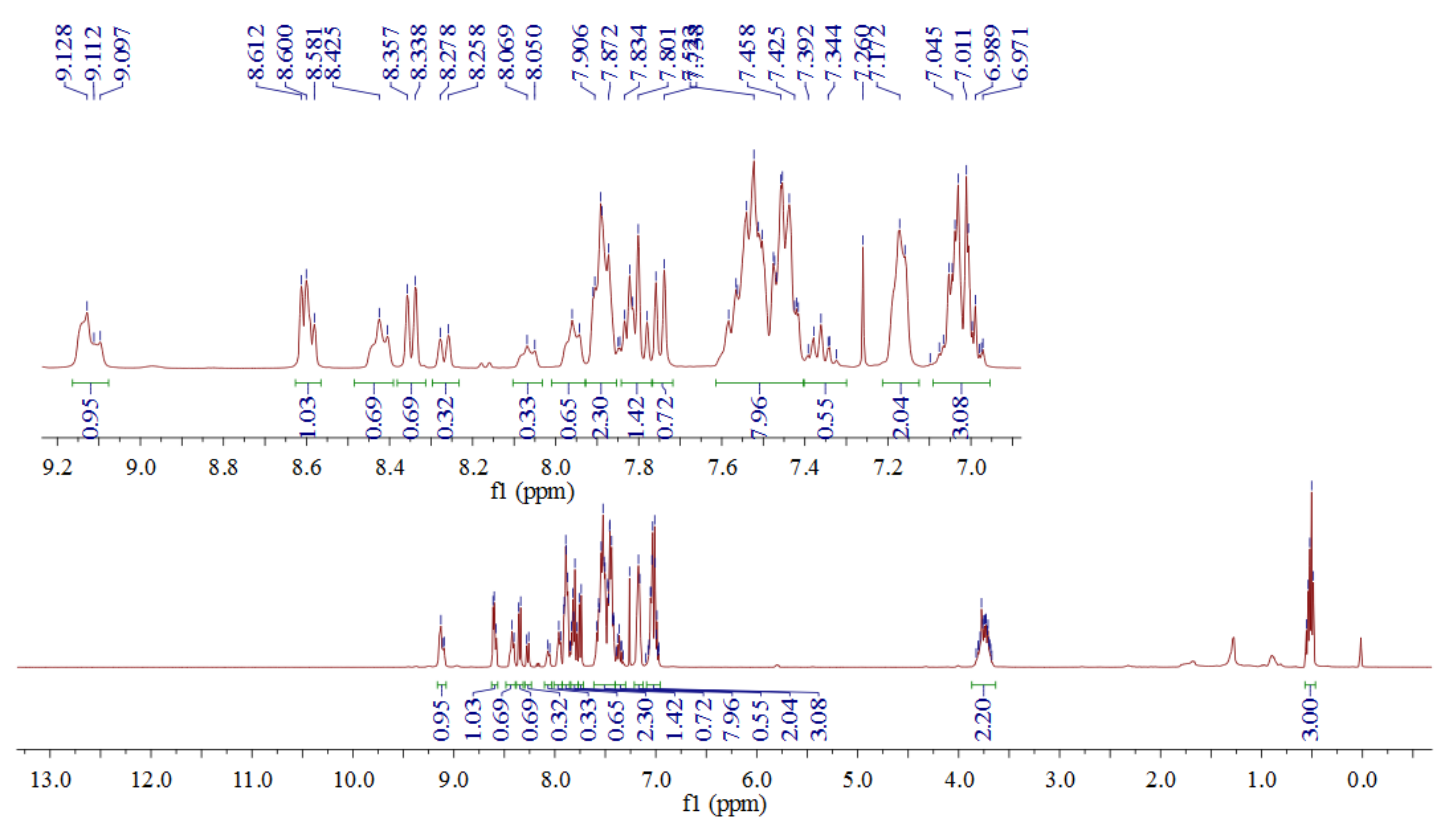


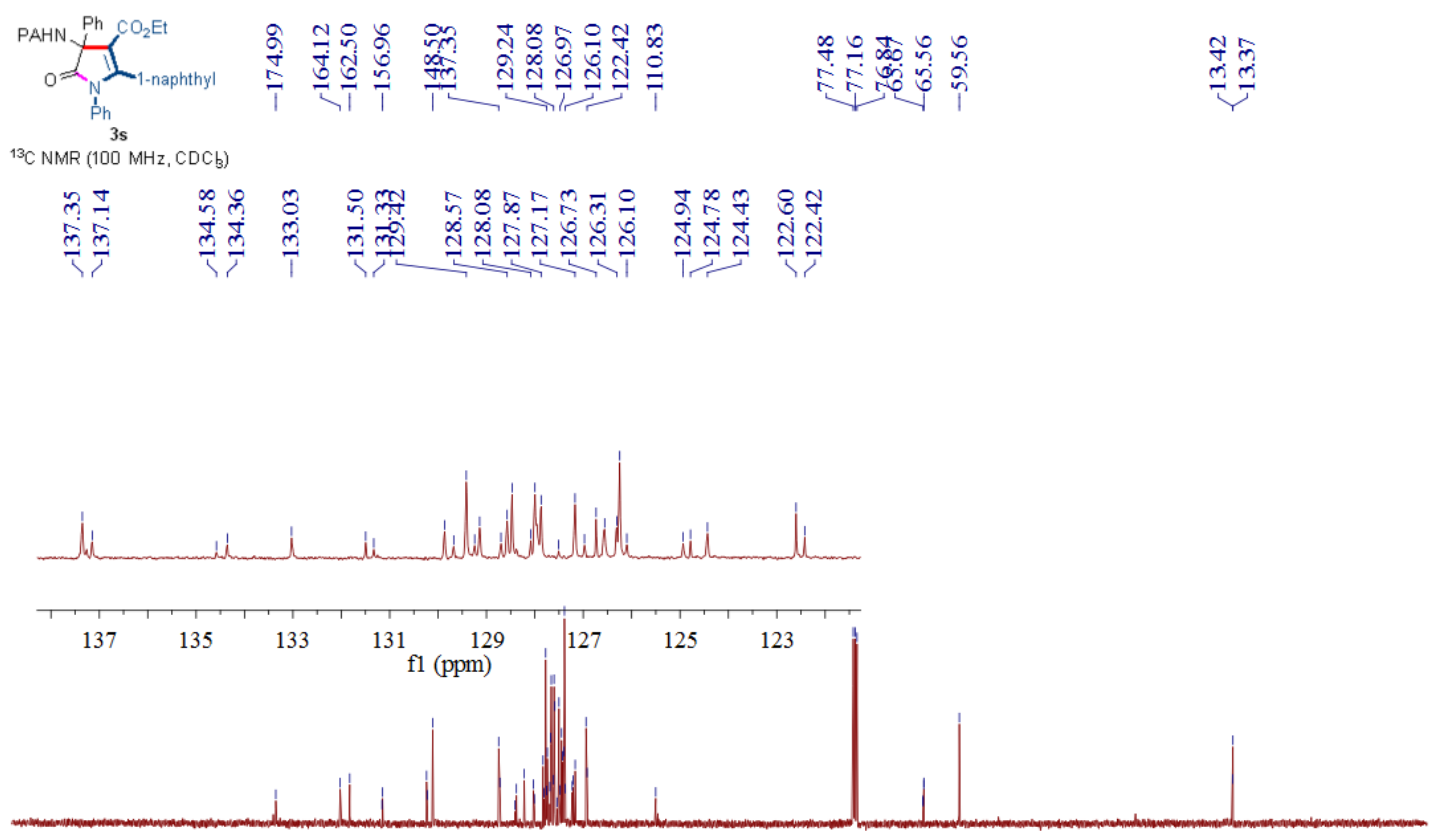

\begin{tabular}{rllllllllllllllll}
\hline 210 & 190 & 170 & 150 & 130 & 110 & 90 & 80 & 70 & 60 & 50 & 40 & 30 & 20 & 10 & 0 & -10
\end{tabular}

${ }^{1} \mathrm{H}$ NMR and ${ }^{13} \mathrm{C}$ NMR spectra for compound 3 s (using $\mathrm{CDCl}_{3}$ as solvent)

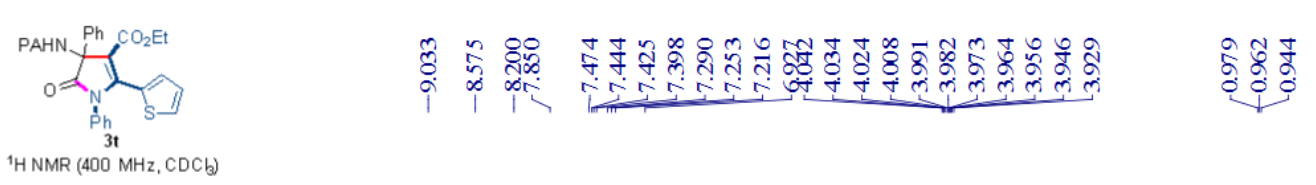

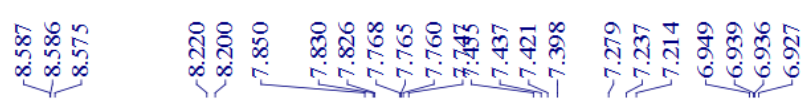

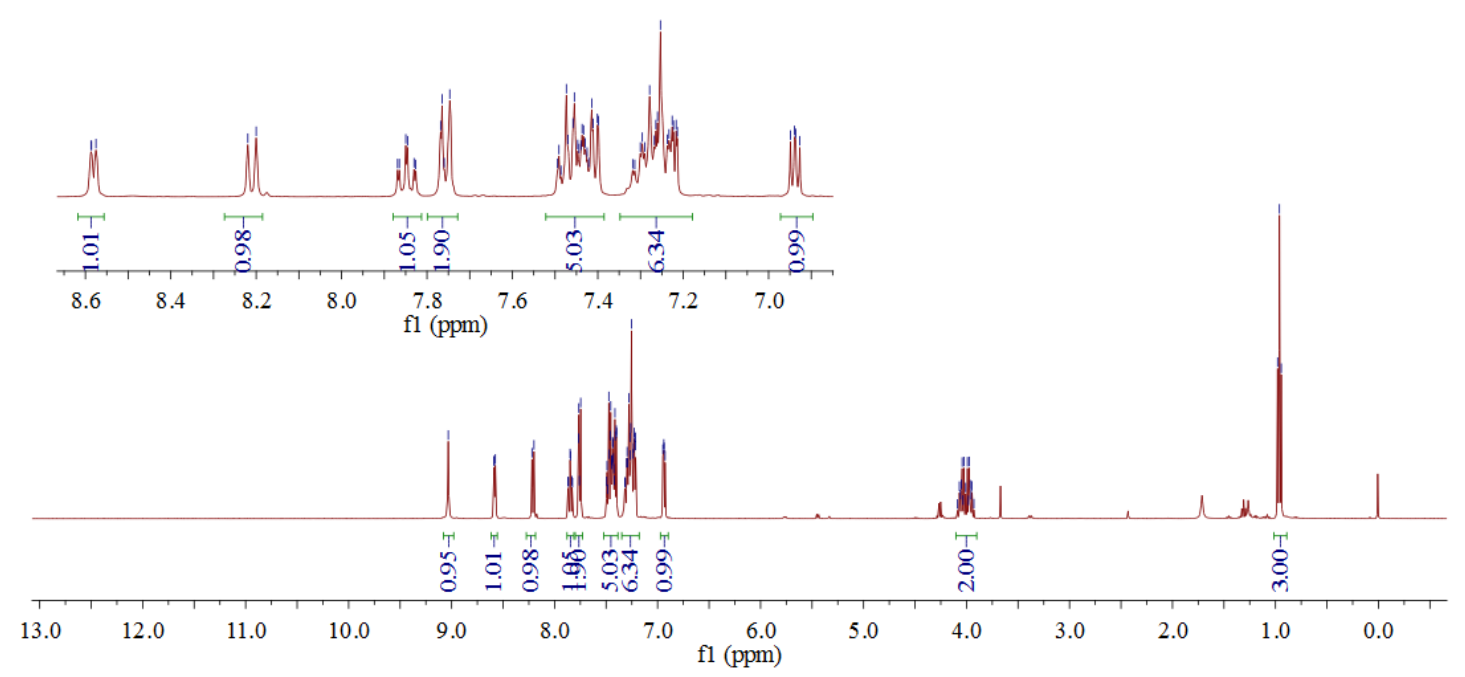




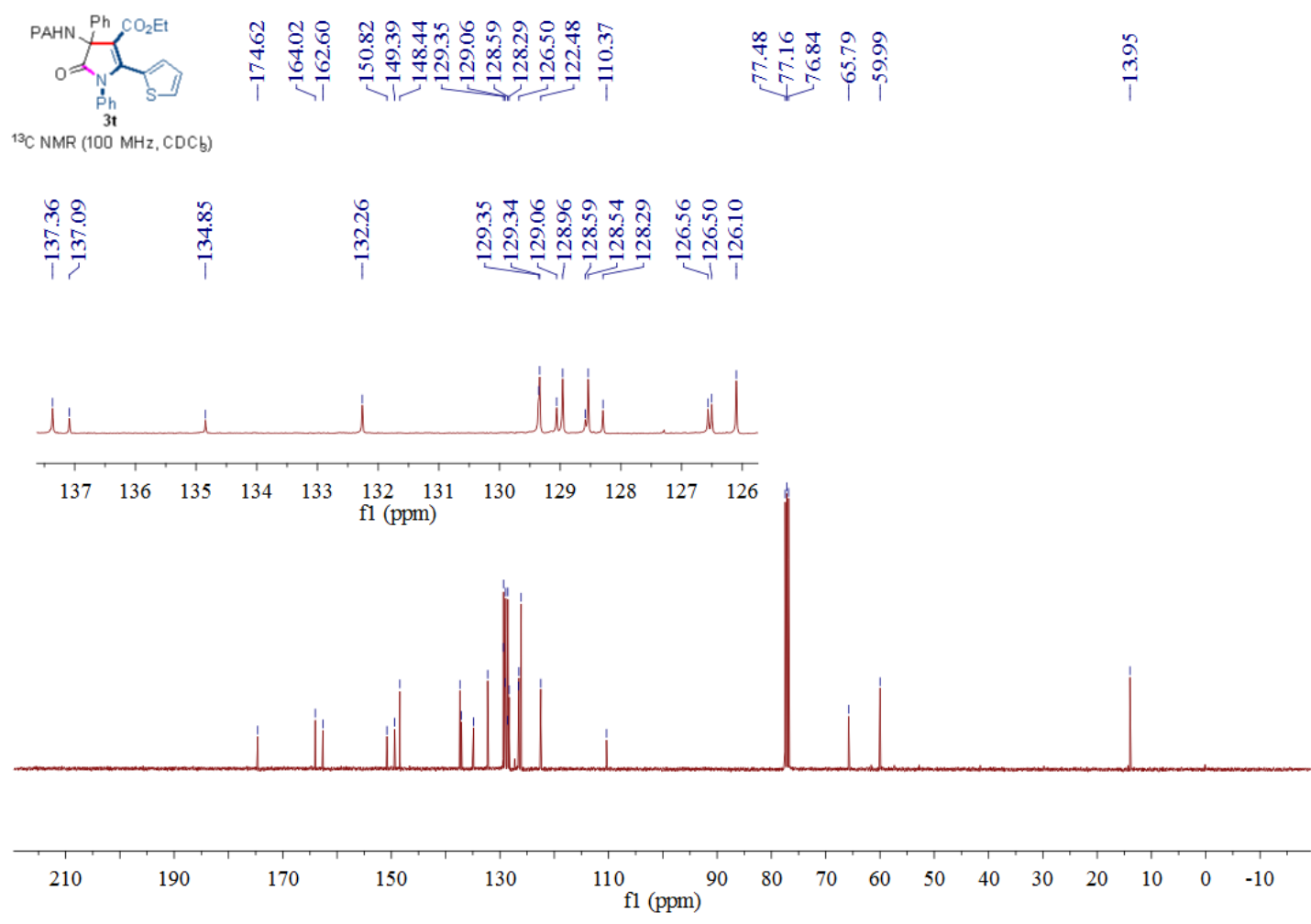

${ }^{1} \mathrm{H}$ NMR and ${ }^{13} \mathrm{C}$ NMR spectra for compound $3 t$ (using $\mathrm{CDCl}_{3}$ as solvent)

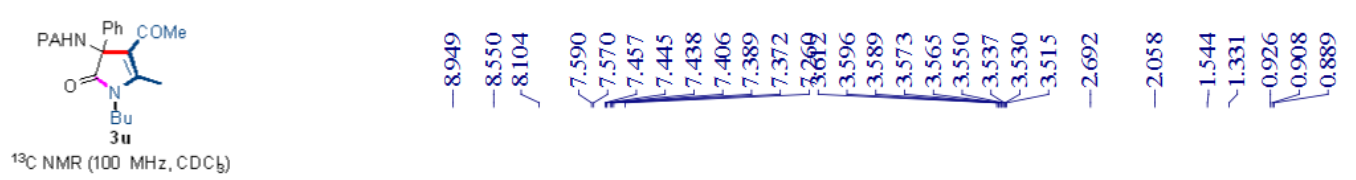

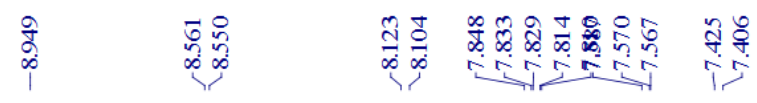

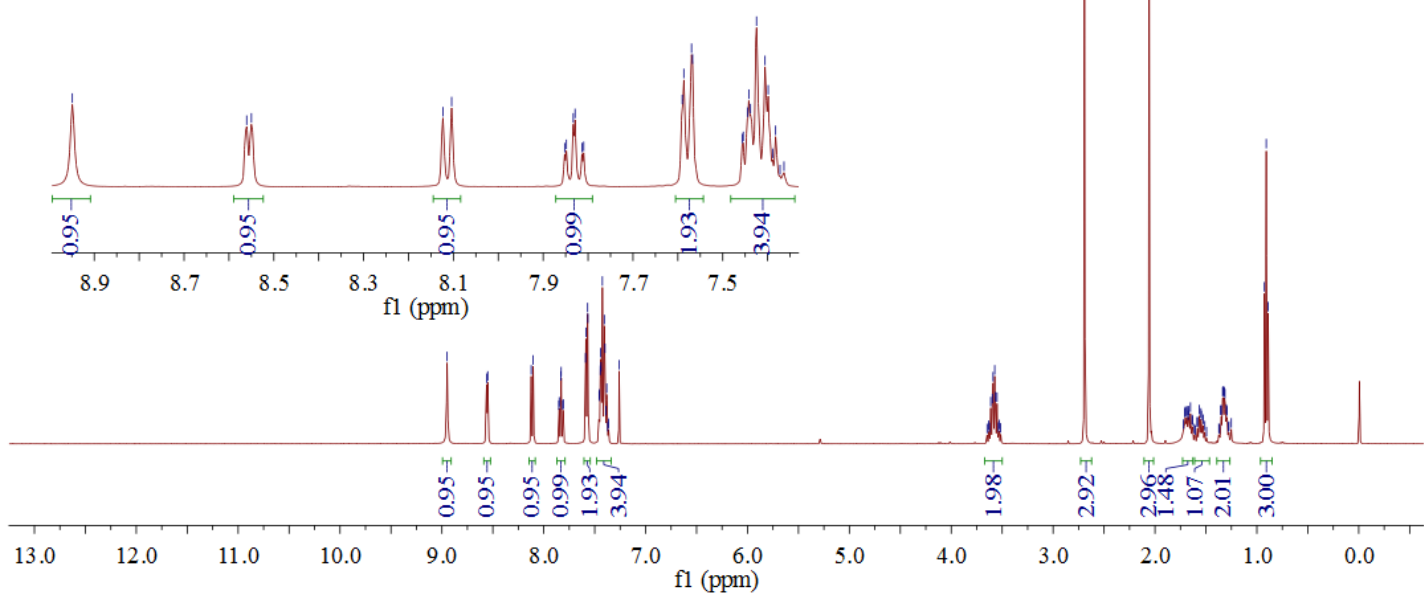



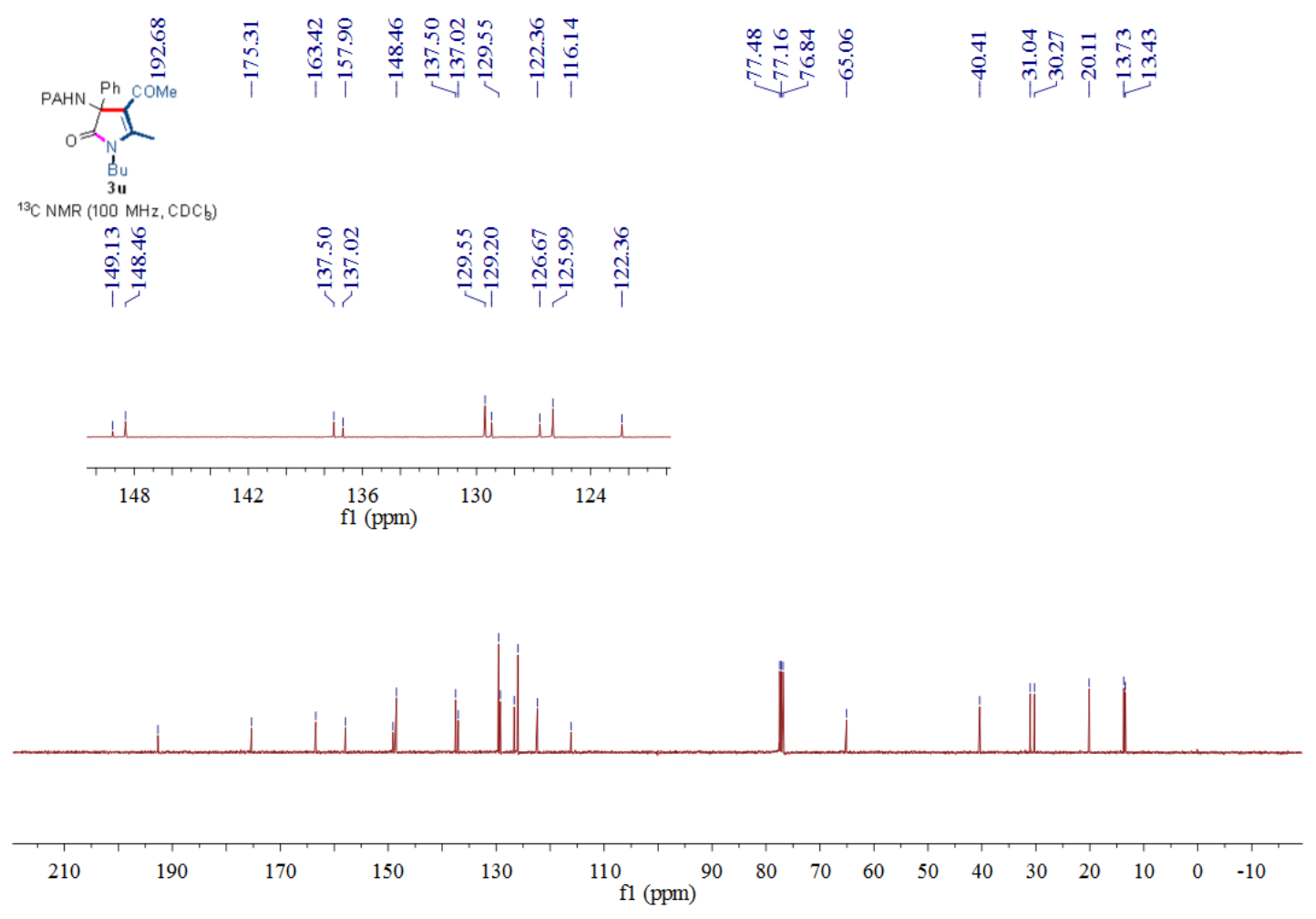

${ }^{1} \mathrm{H}$ NMR and ${ }^{13} \mathrm{C}$ NMR spectra for compound $\mathbf{3 u}$ (using $\mathrm{CDCl}_{3}$ as solvent)

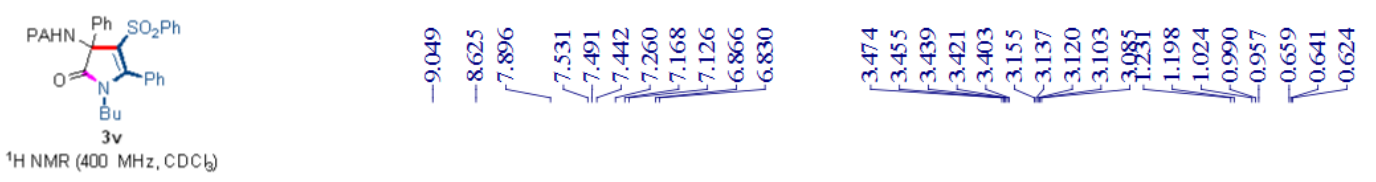

$\begin{array}{lll}0 & 0 \\ 0 & 0\end{array}$
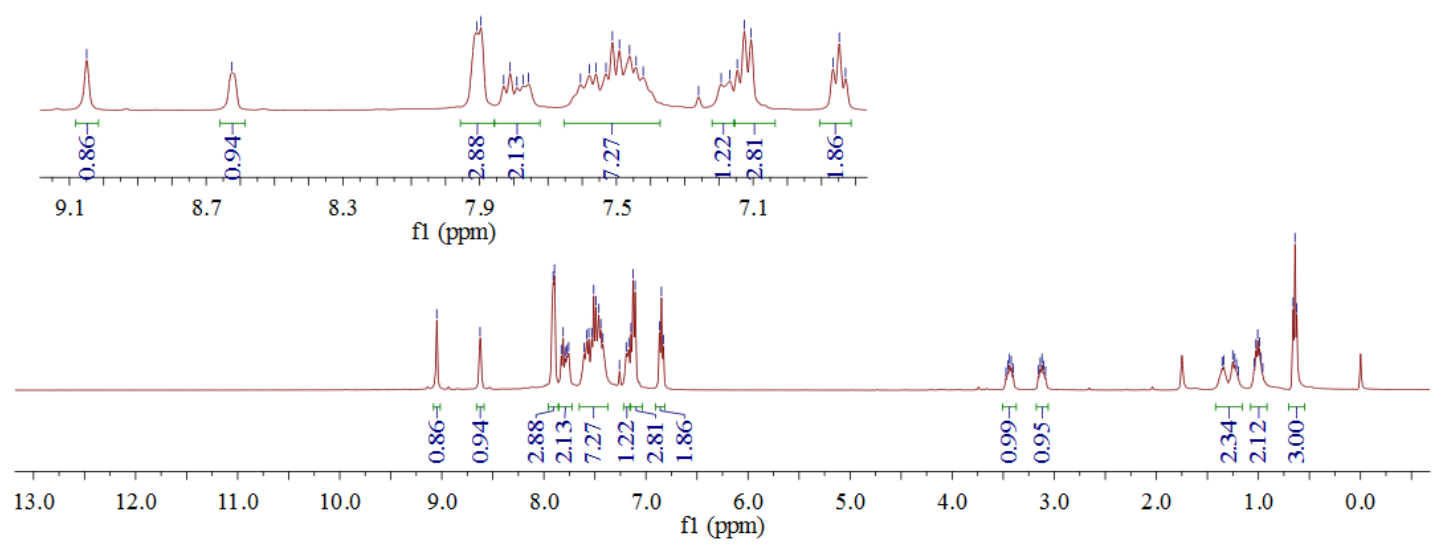


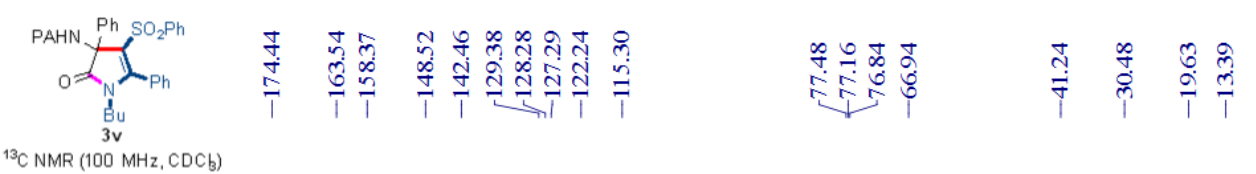

${ }^{13} \mathrm{C}$ NMR (100 MHz $\left.\mathrm{CDCH}\right)$
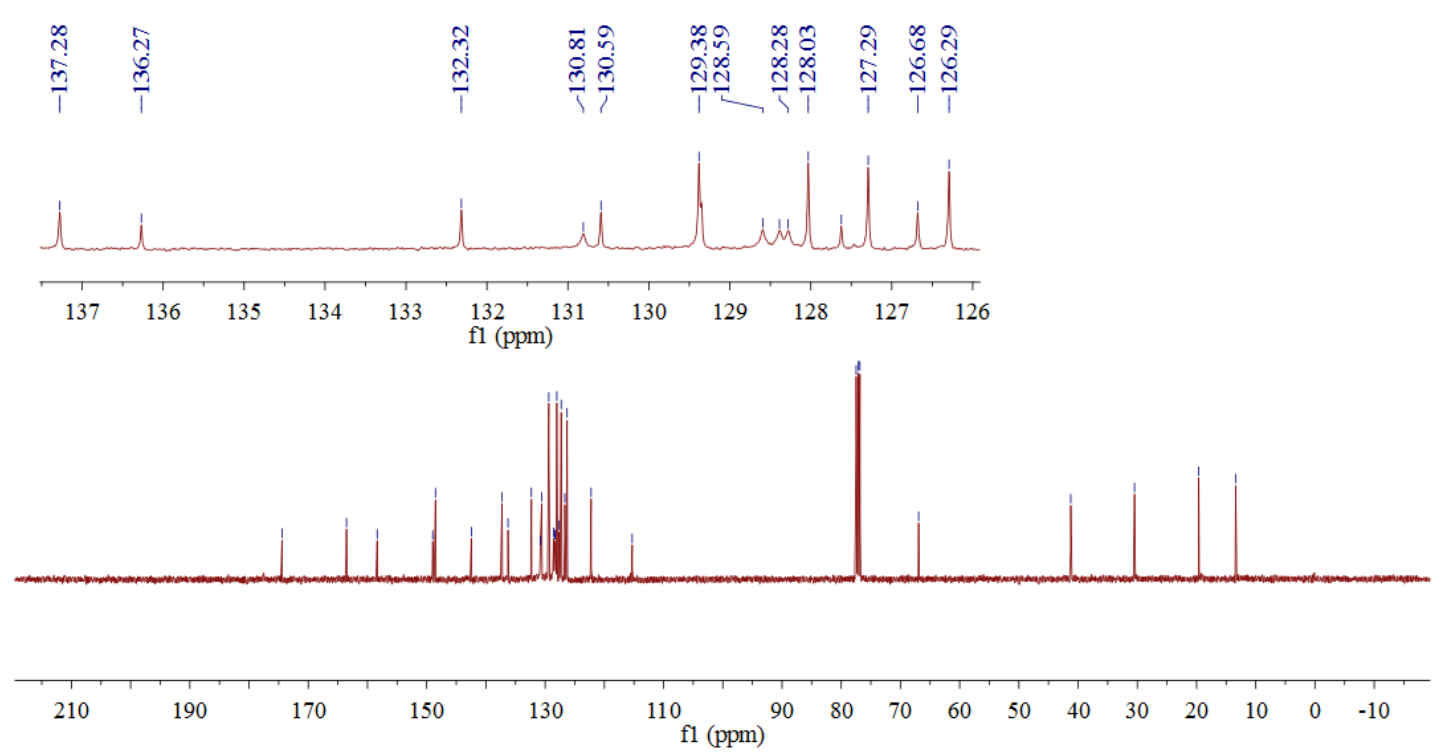

${ }^{1} \mathrm{H}$ NMR and ${ }^{13} \mathrm{C}$ NMR spectra for compound $\mathbf{3 v}$ (using $\mathrm{CDCl}_{3}$ as solvent)

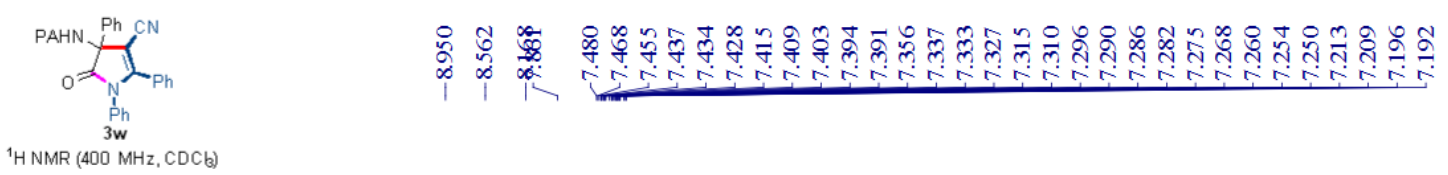

$\begin{array}{lll}\infty & \infty \\ 0 & \infty\end{array}$
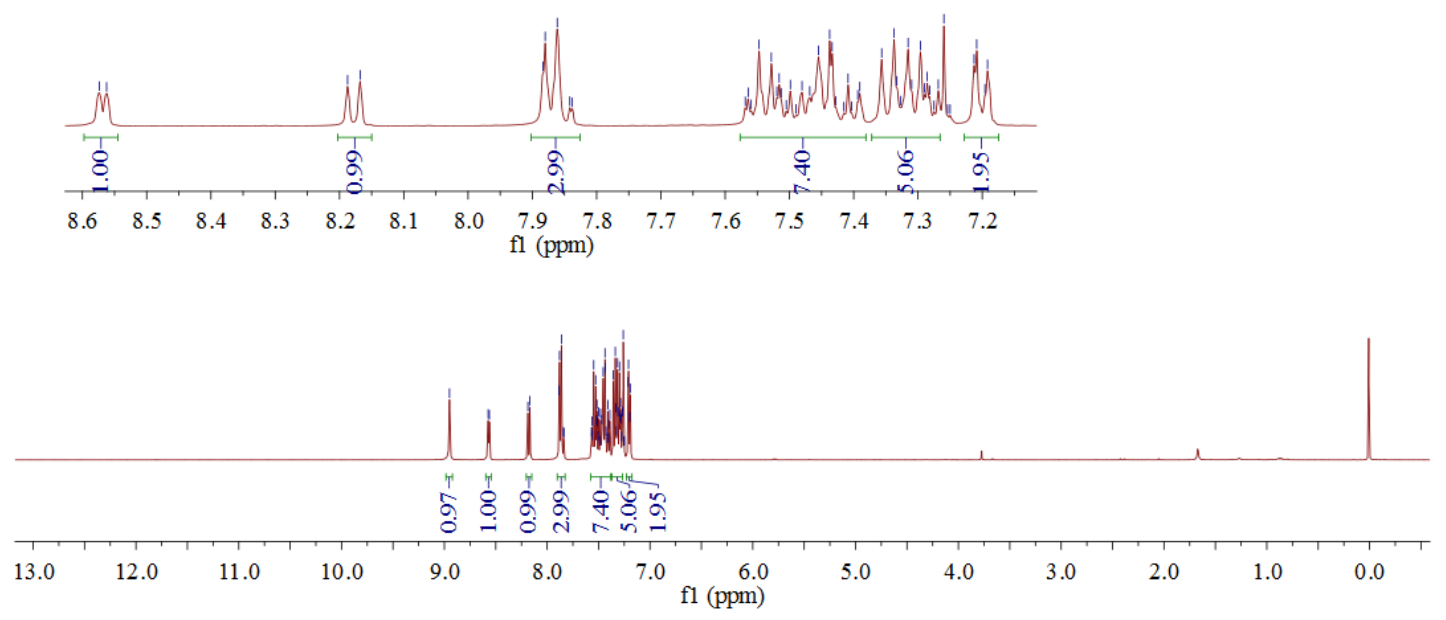

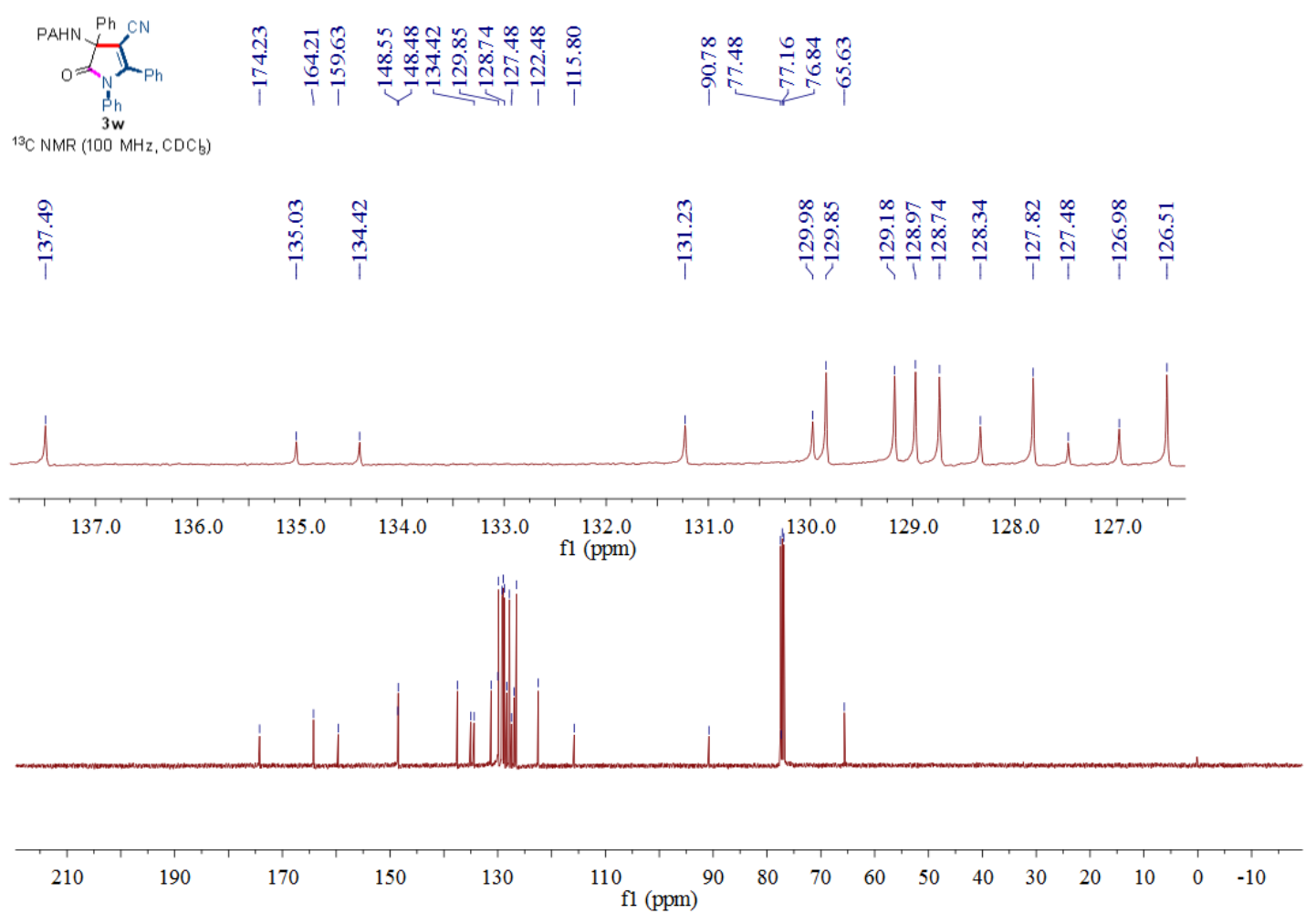

${ }^{1} \mathrm{H}$ NMR and ${ }^{13} \mathrm{C}$ NMR spectra for compound $\mathbf{3 w}$ (using $\mathrm{CDCl}_{3}$ as solvent)

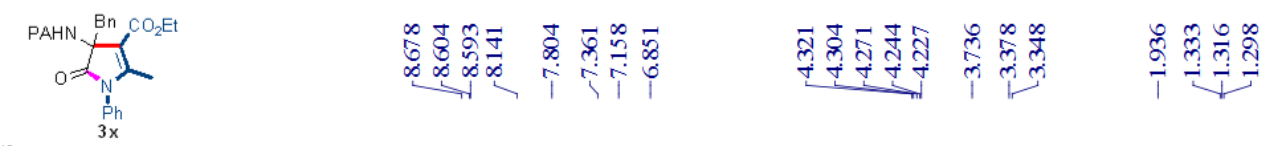

${ }^{13} \mathrm{C}$ NMR $(100 \mathrm{MHz}, \mathrm{CDC}$ )

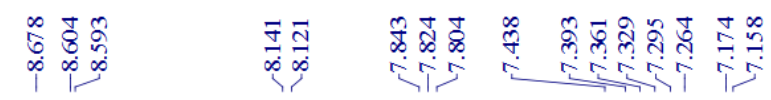
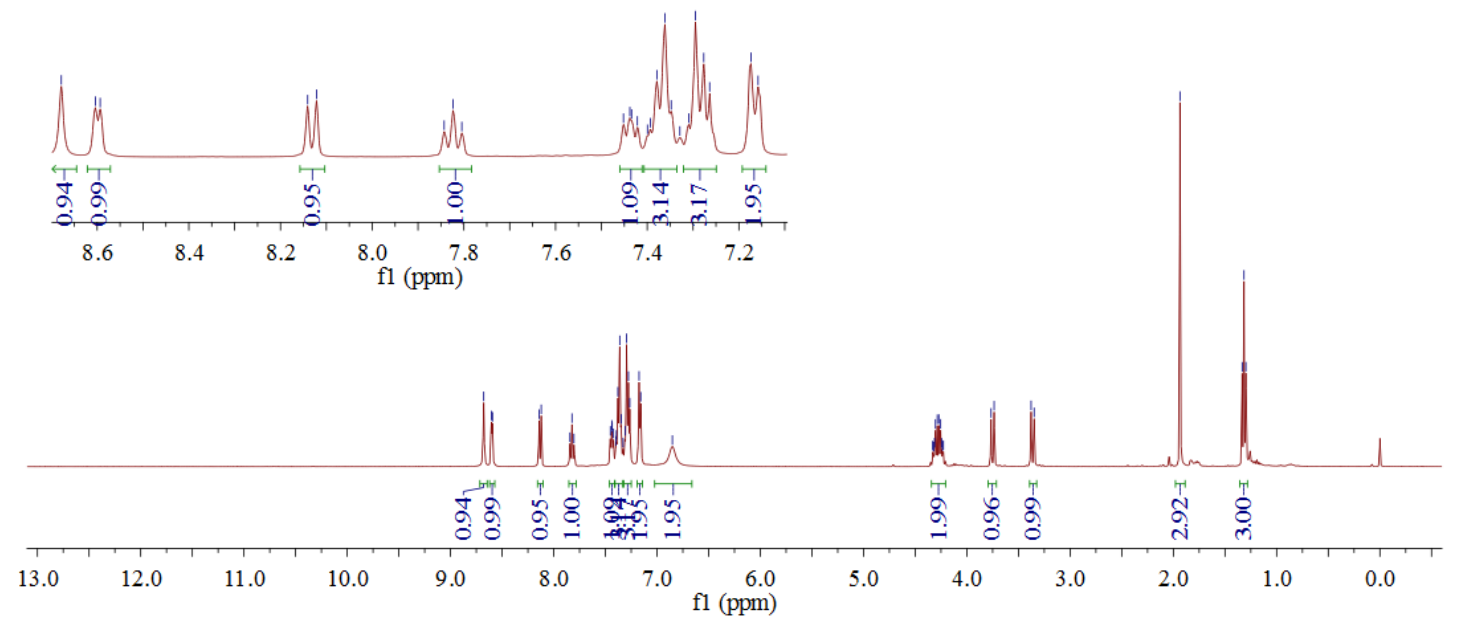


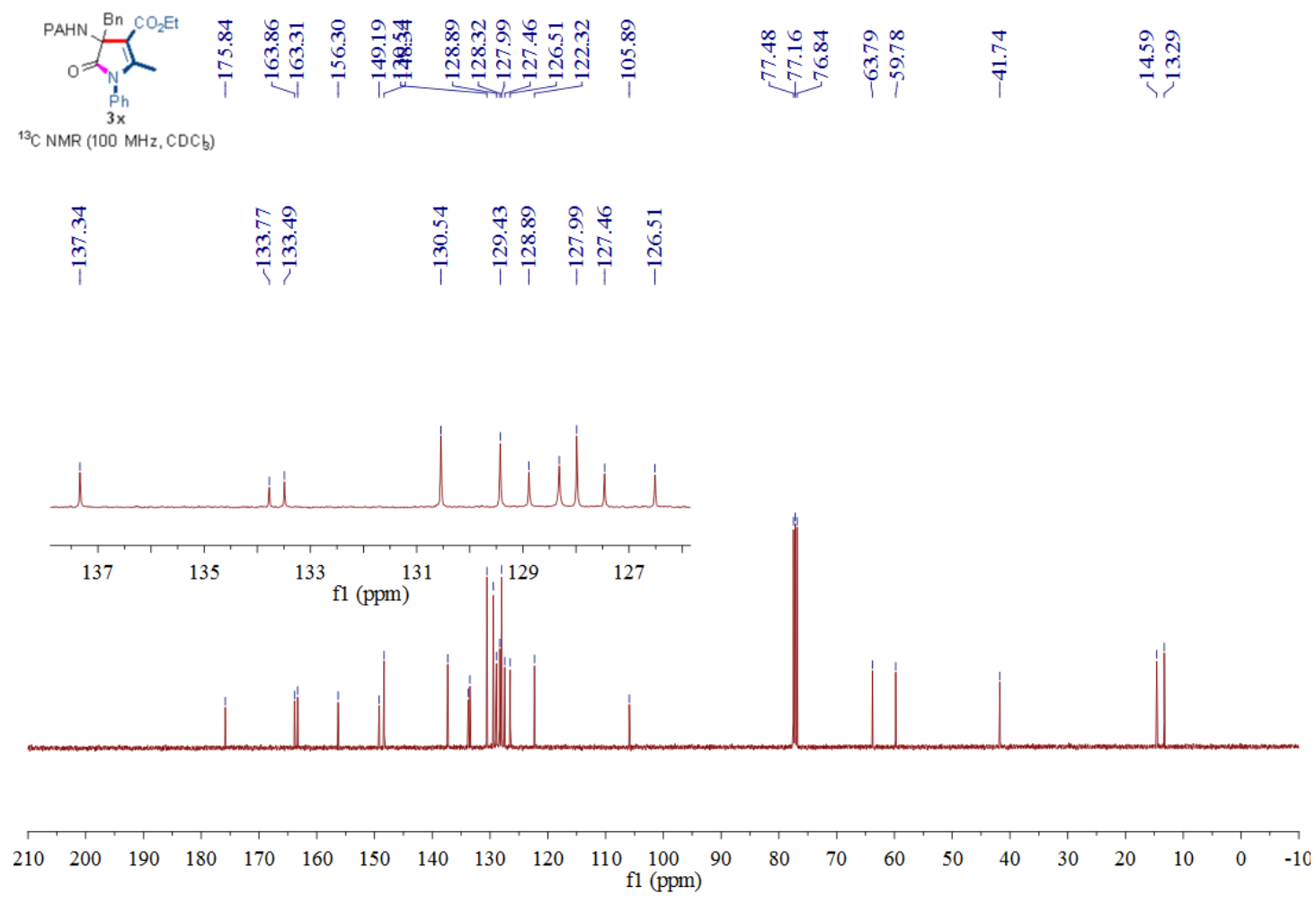

${ }^{1} \mathrm{H}$ NMR and ${ }^{13} \mathrm{C}$ NMR spectra for compound $\mathbf{3 x}$ (using $\mathrm{CDCl}_{3}$ as solvent)

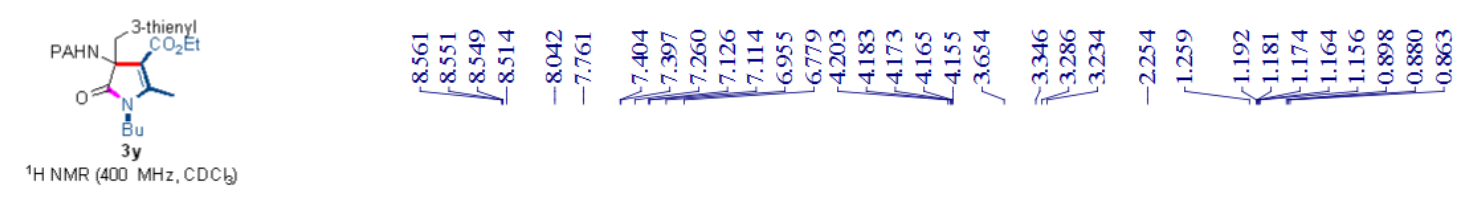

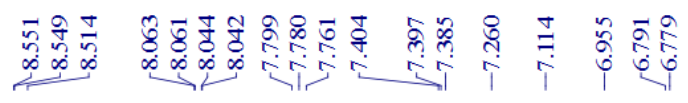
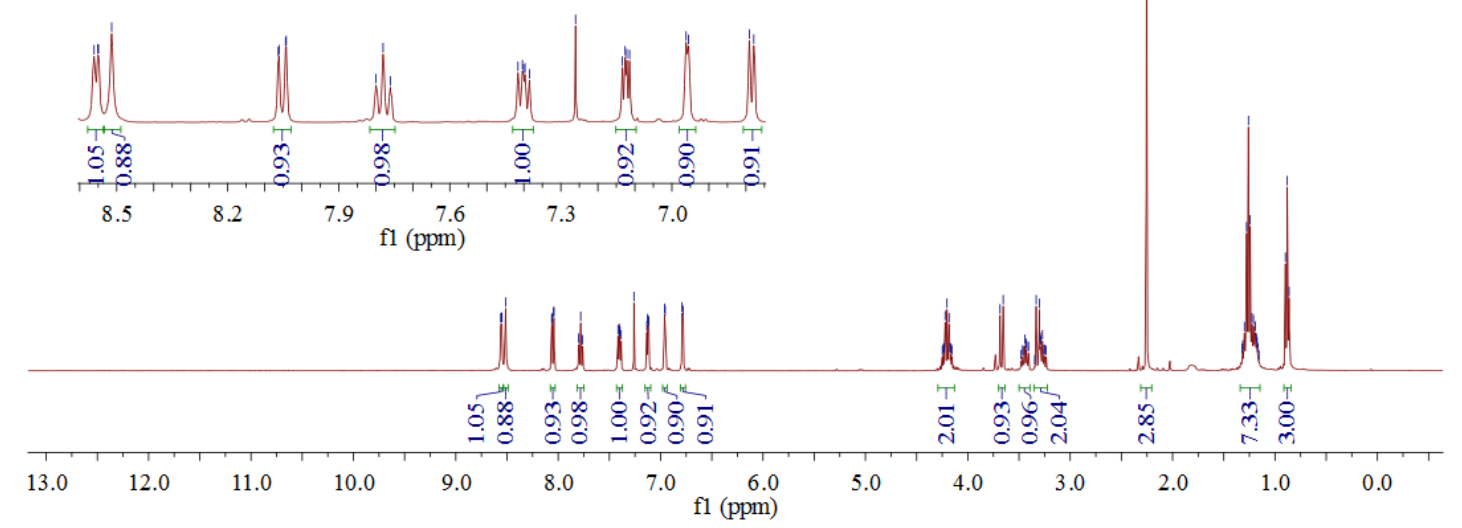

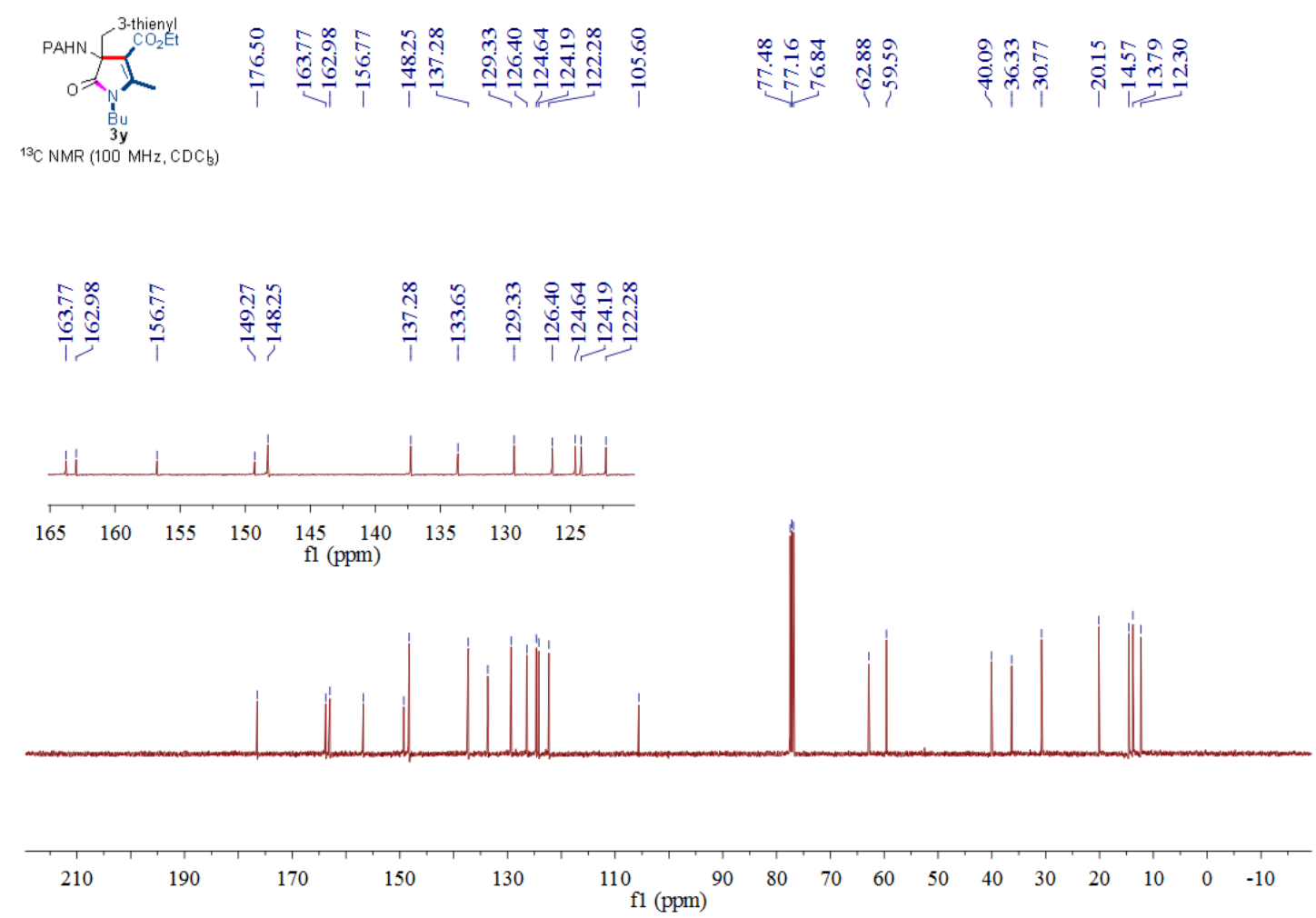

${ }^{1} \mathrm{H}$ NMR and ${ }^{13} \mathrm{C}$ NMR spectra for compound $\mathbf{3 y}$ (using $\mathrm{CDCl}_{3}$ as solvent)

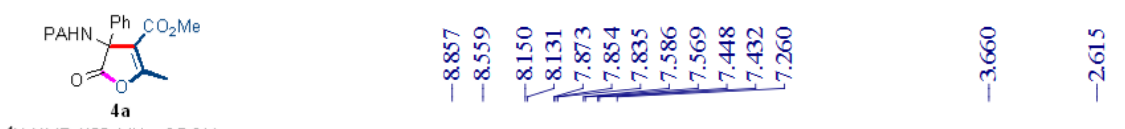

${ }^{1} \mathrm{H} N M R(400 \mathrm{MHz}, \mathrm{CDCb})$
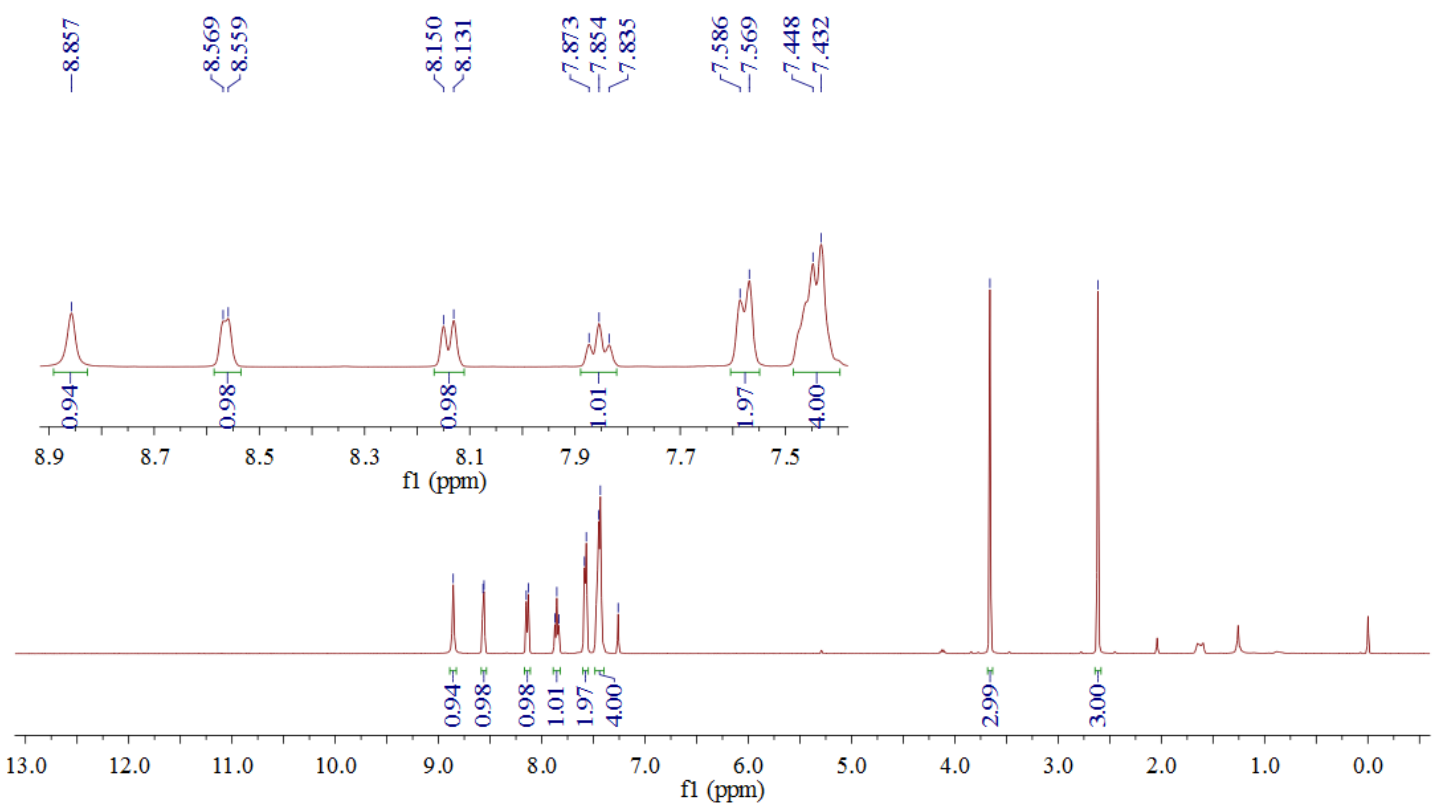


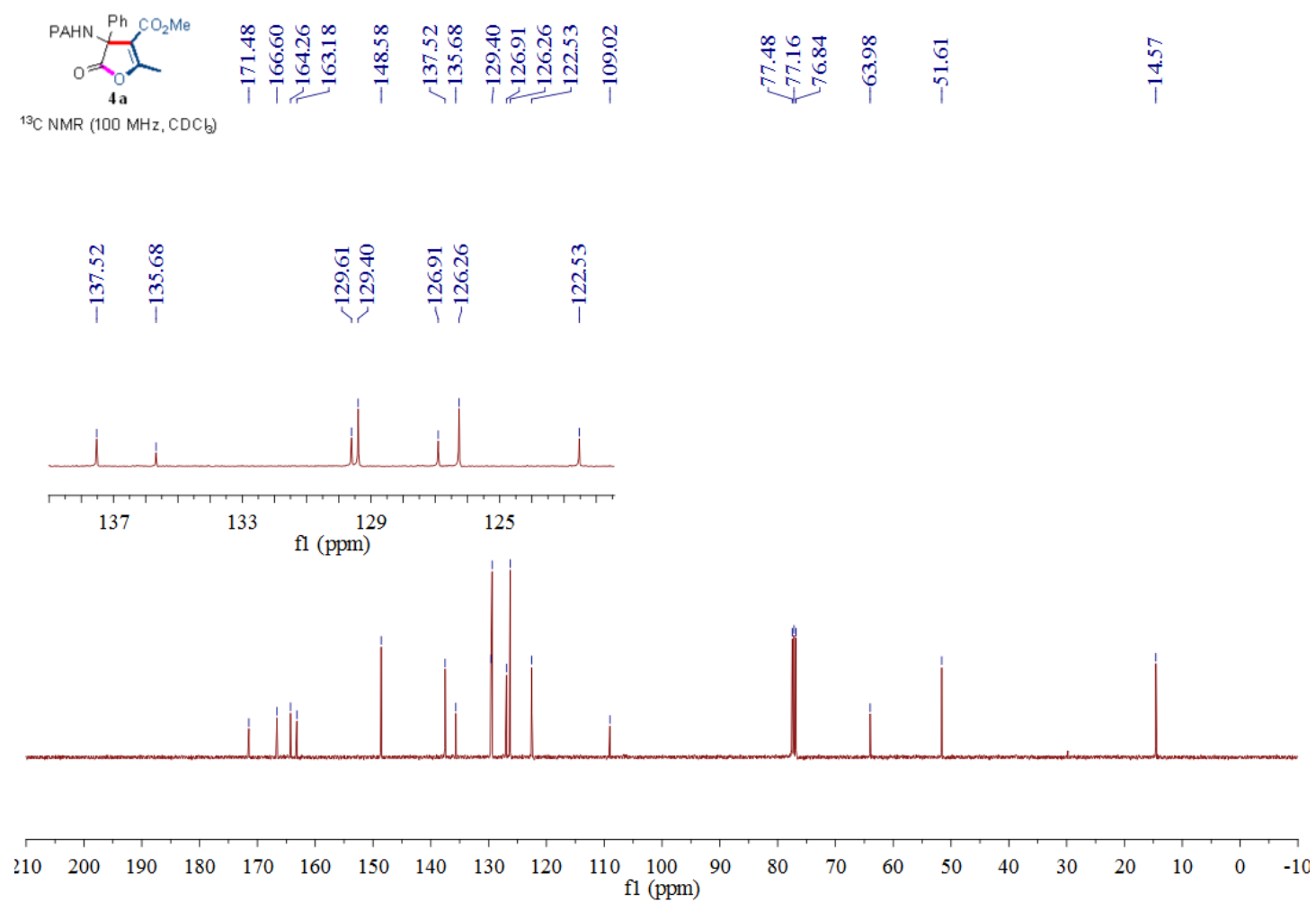

${ }^{1} \mathrm{H}$ NMR and ${ }^{13} \mathrm{C}$ NMR spectra for compound $4 \mathbf{a}$ (using $\mathrm{CDCl}_{3}$ as solvent)

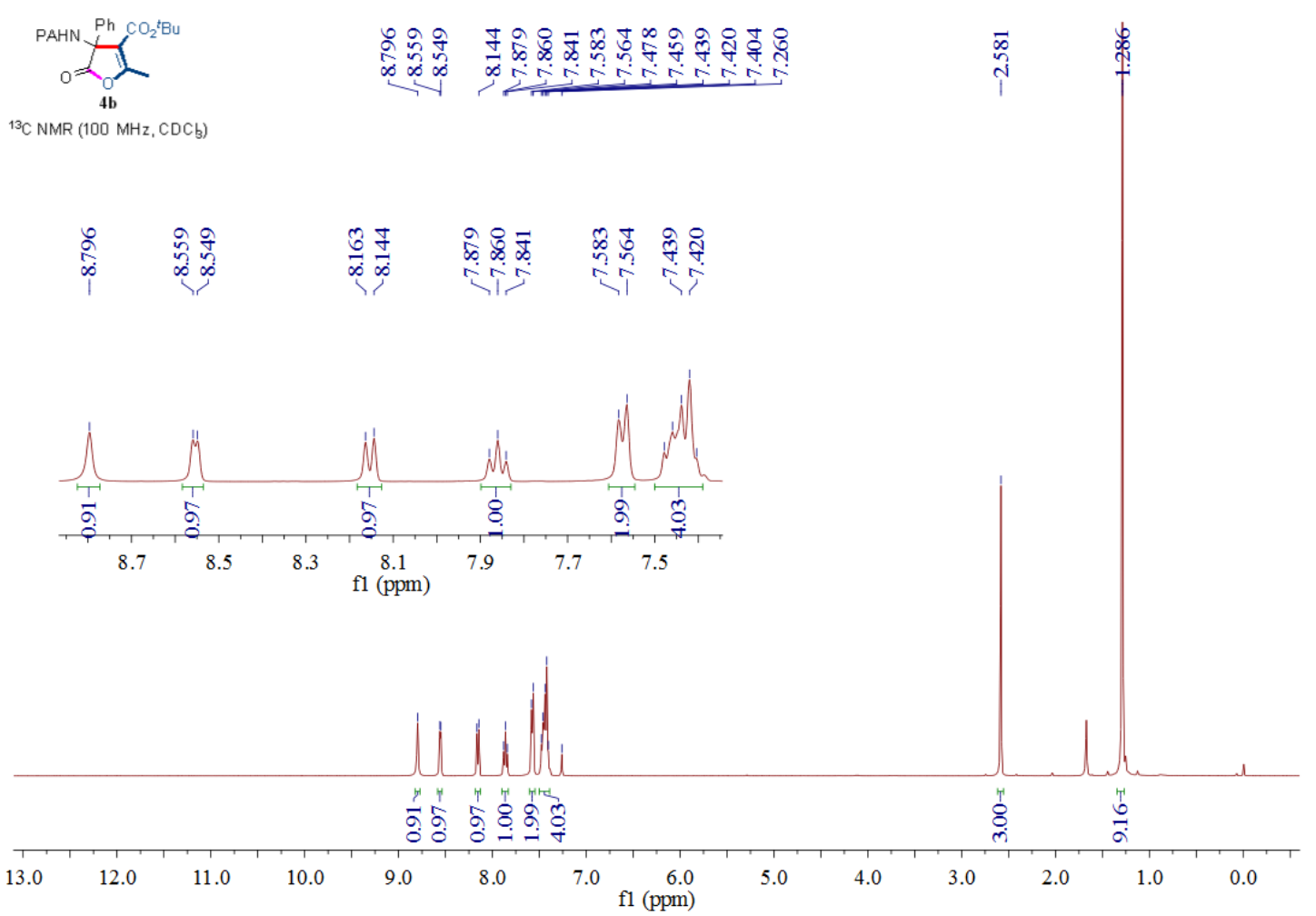



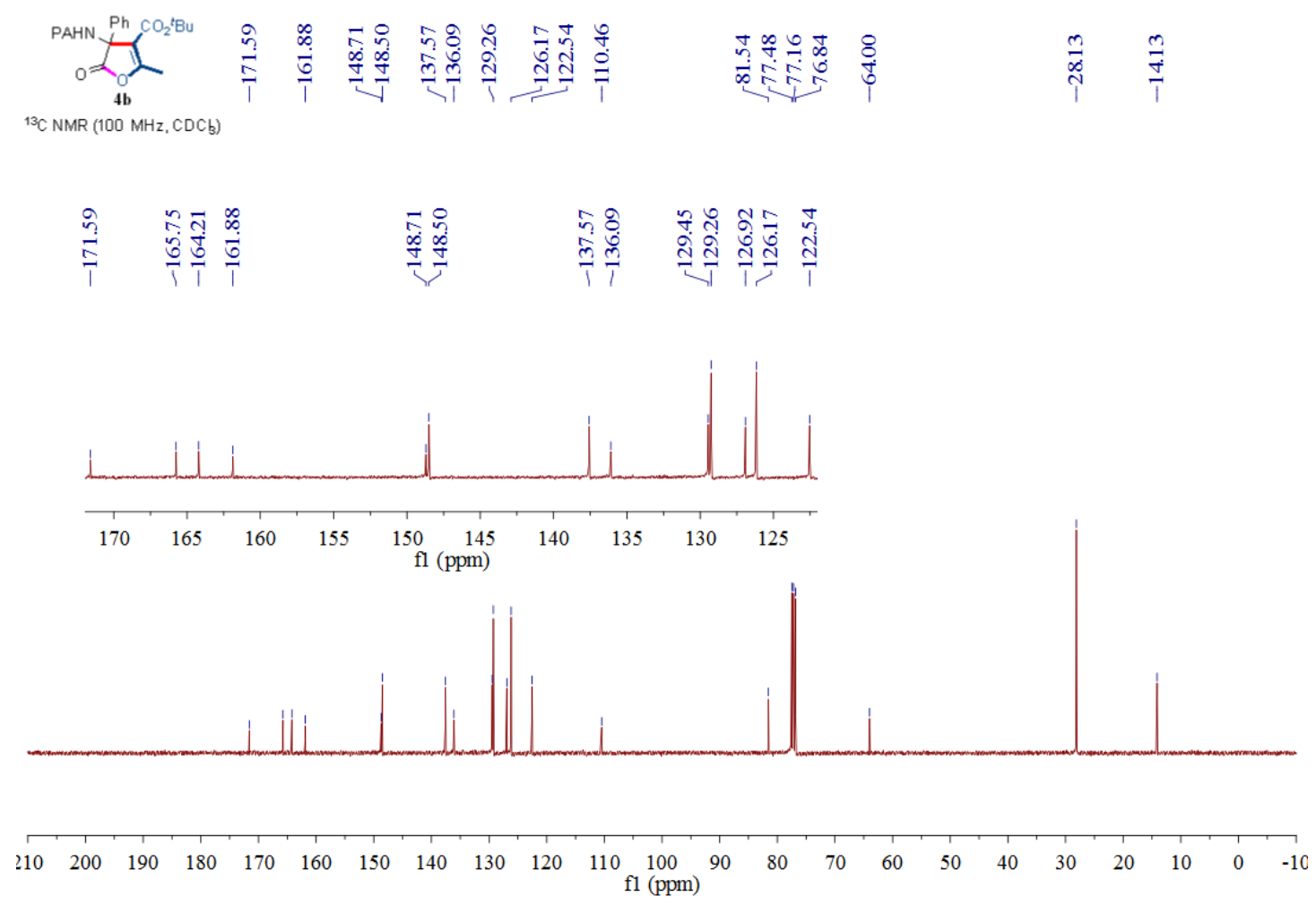

${ }^{1} \mathrm{H}$ NMR and ${ }^{13} \mathrm{C}$ NMR spectra for compound $\mathbf{4 b}$ (using $\mathrm{CDCl}_{3}$ as solvent)

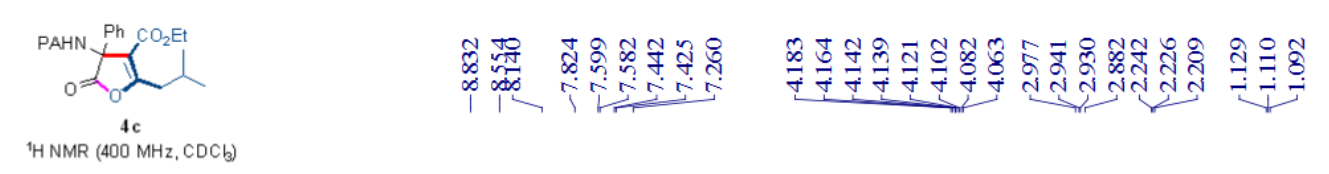

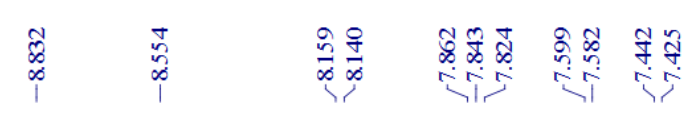
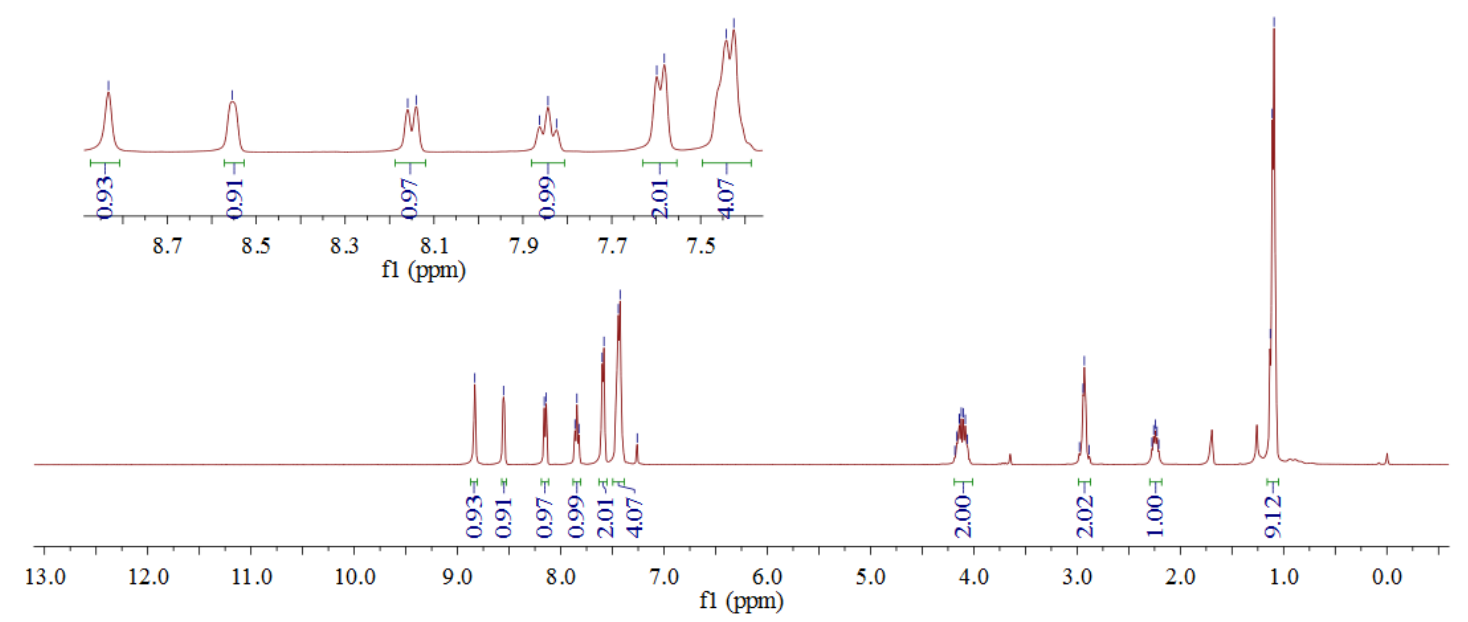


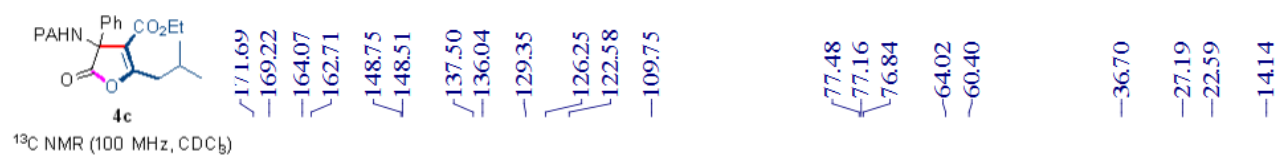

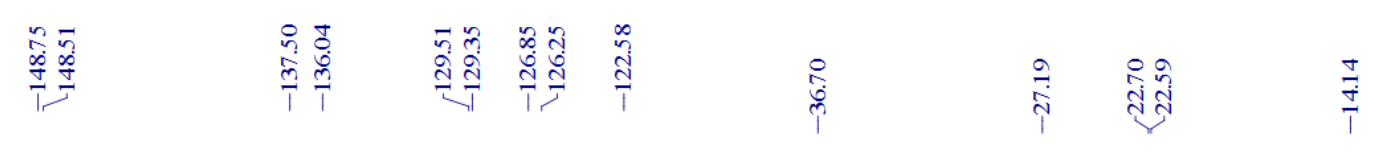
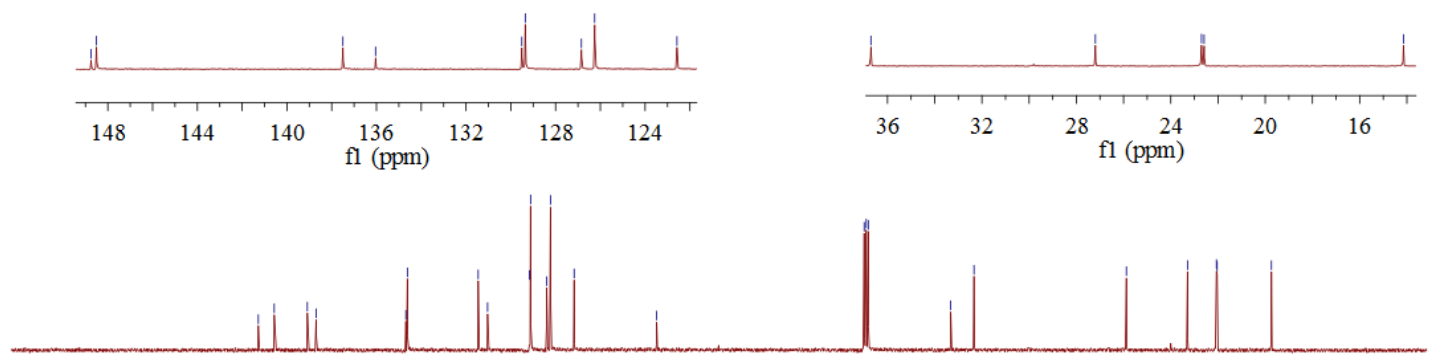

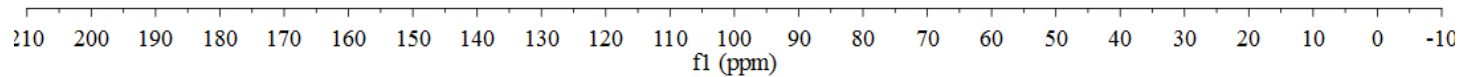

${ }^{1} \mathrm{H}$ NMR and ${ }^{13} \mathrm{C}$ NMR spectra for compound $4 \mathbf{c}$ (using $\mathrm{CDCl}_{3}$ as solvent)
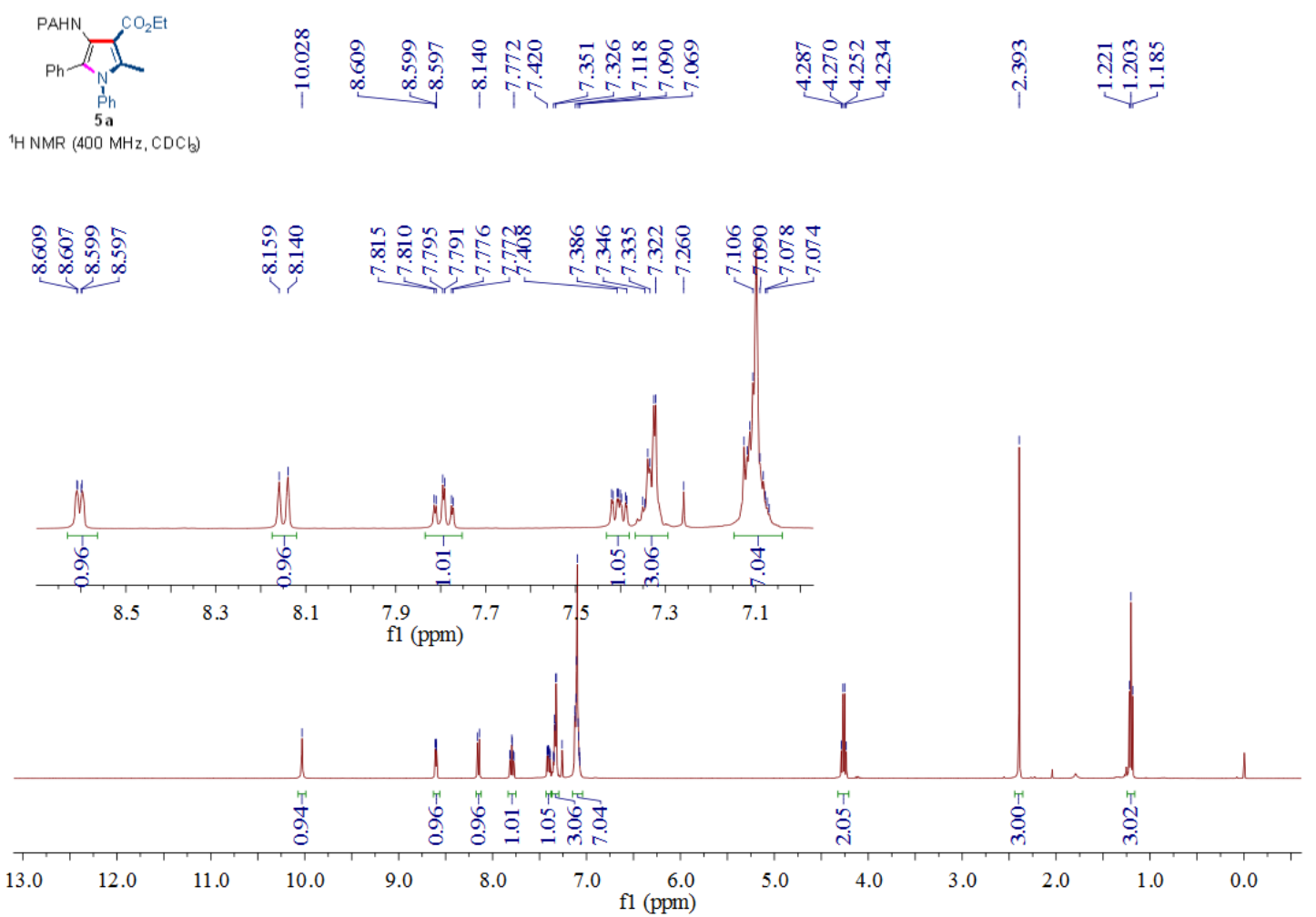


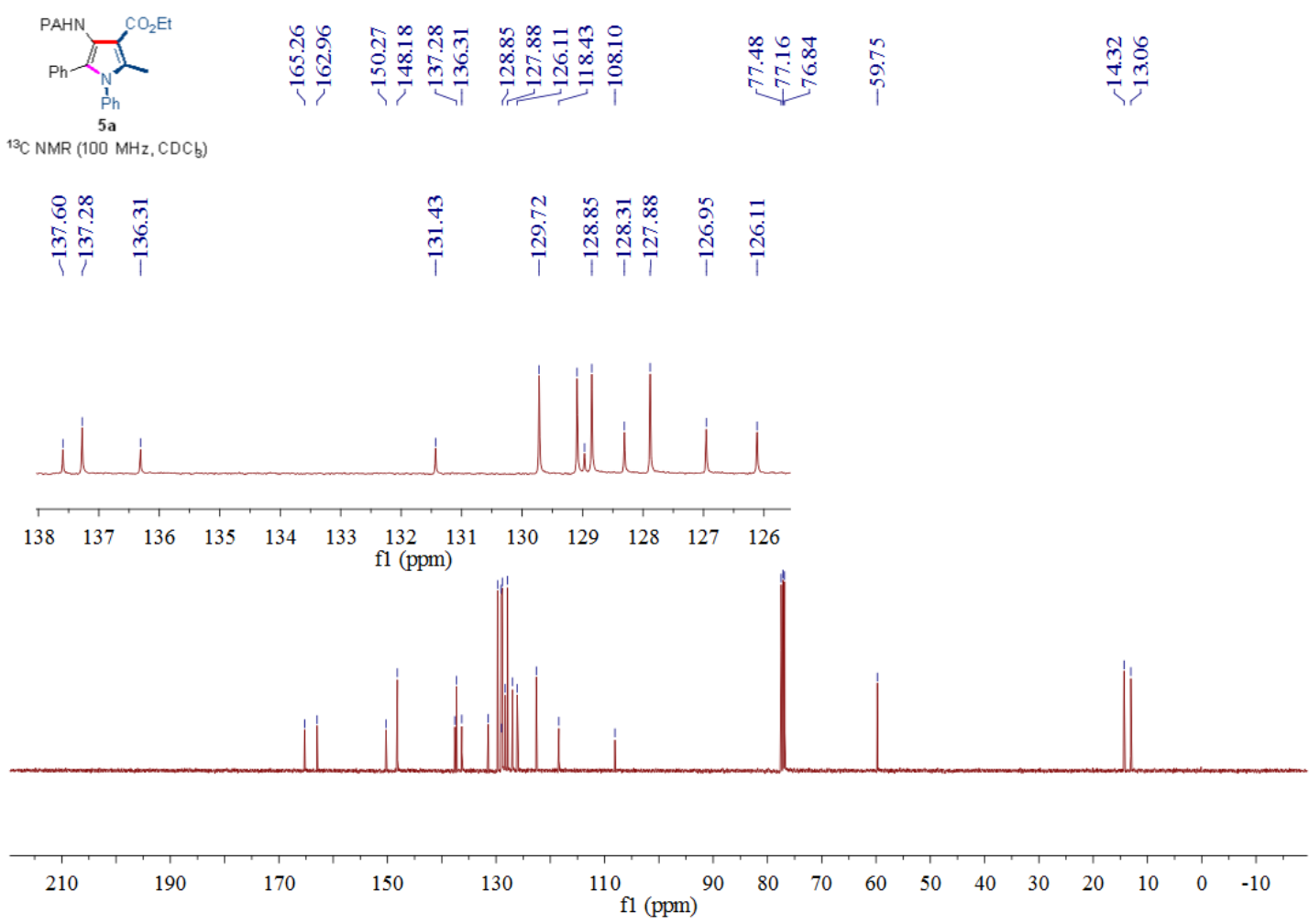

${ }^{1} \mathrm{H}$ NMR and ${ }^{13} \mathrm{C}$ NMR spectra for compound 5a (using $\mathrm{CDCl}_{3}$ as solvent)
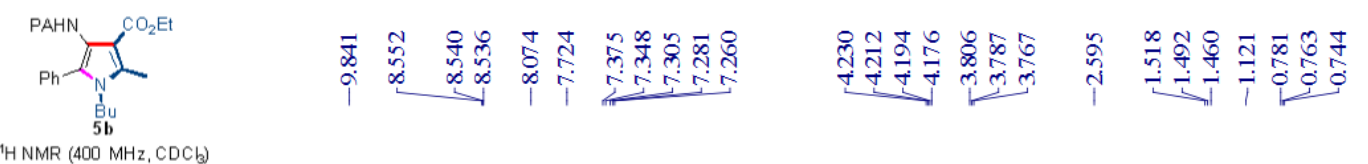

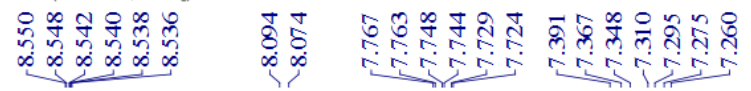
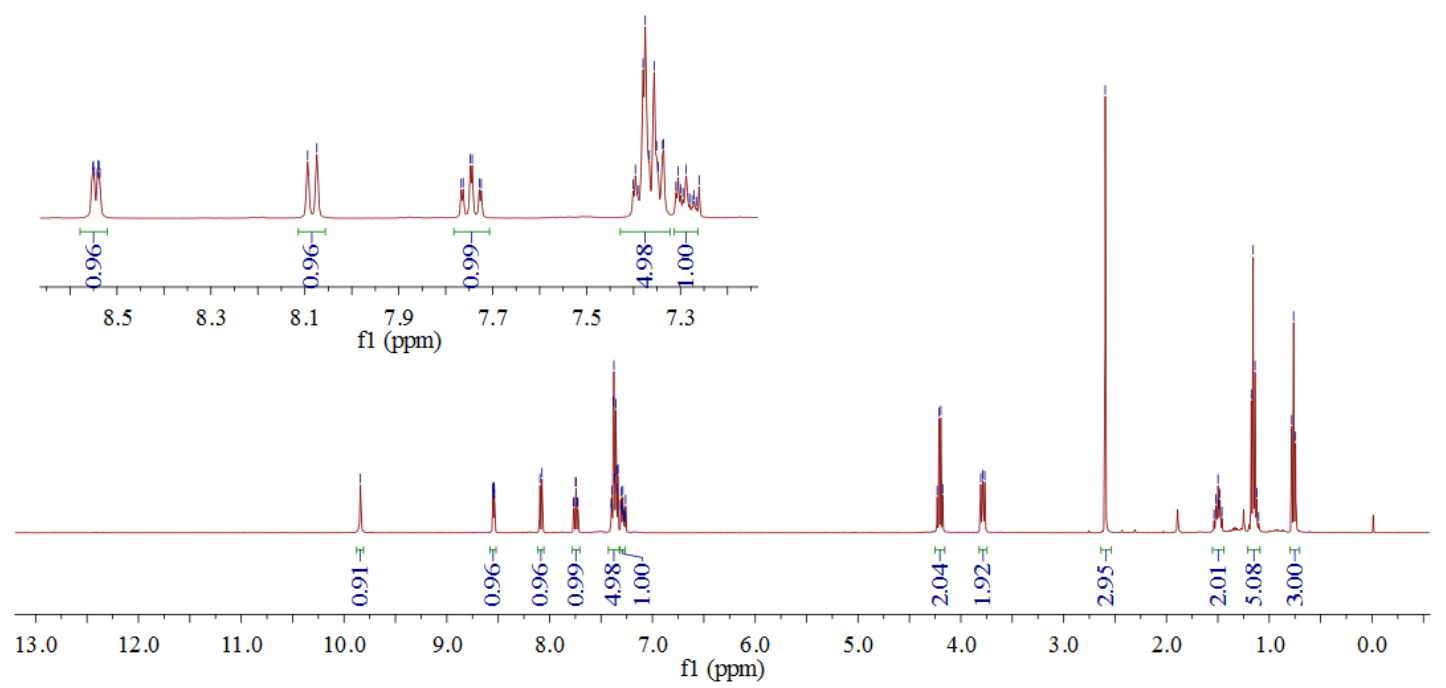


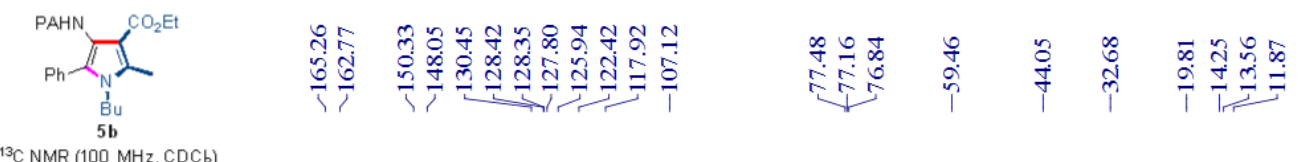

${ }^{13} \mathrm{C}$ NMR $(100 \mathrm{MHz}, \mathrm{CDC}$ )

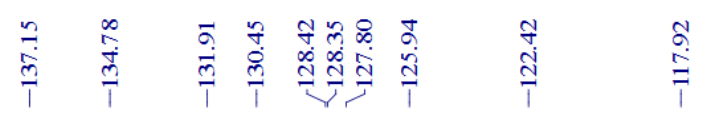

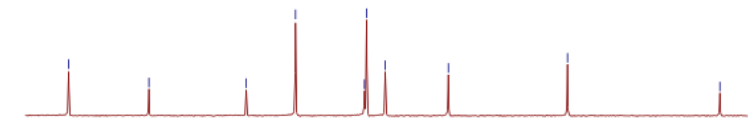

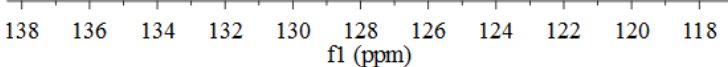
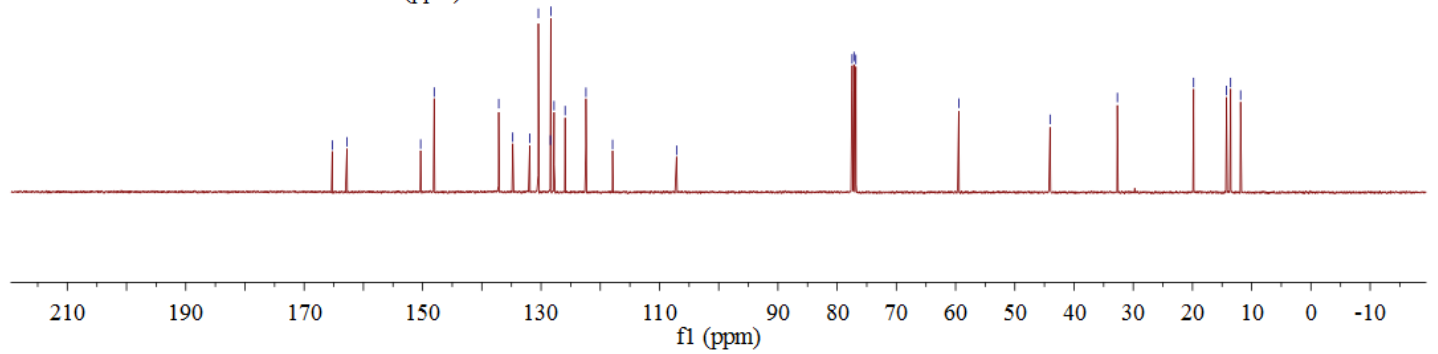

${ }^{1} \mathrm{H} \mathrm{NMR}$ and ${ }^{13} \mathrm{C}$ NMR spectra for compound $\mathbf{5 b}$ (using $\mathrm{CDCl}_{3}$ as solvent)
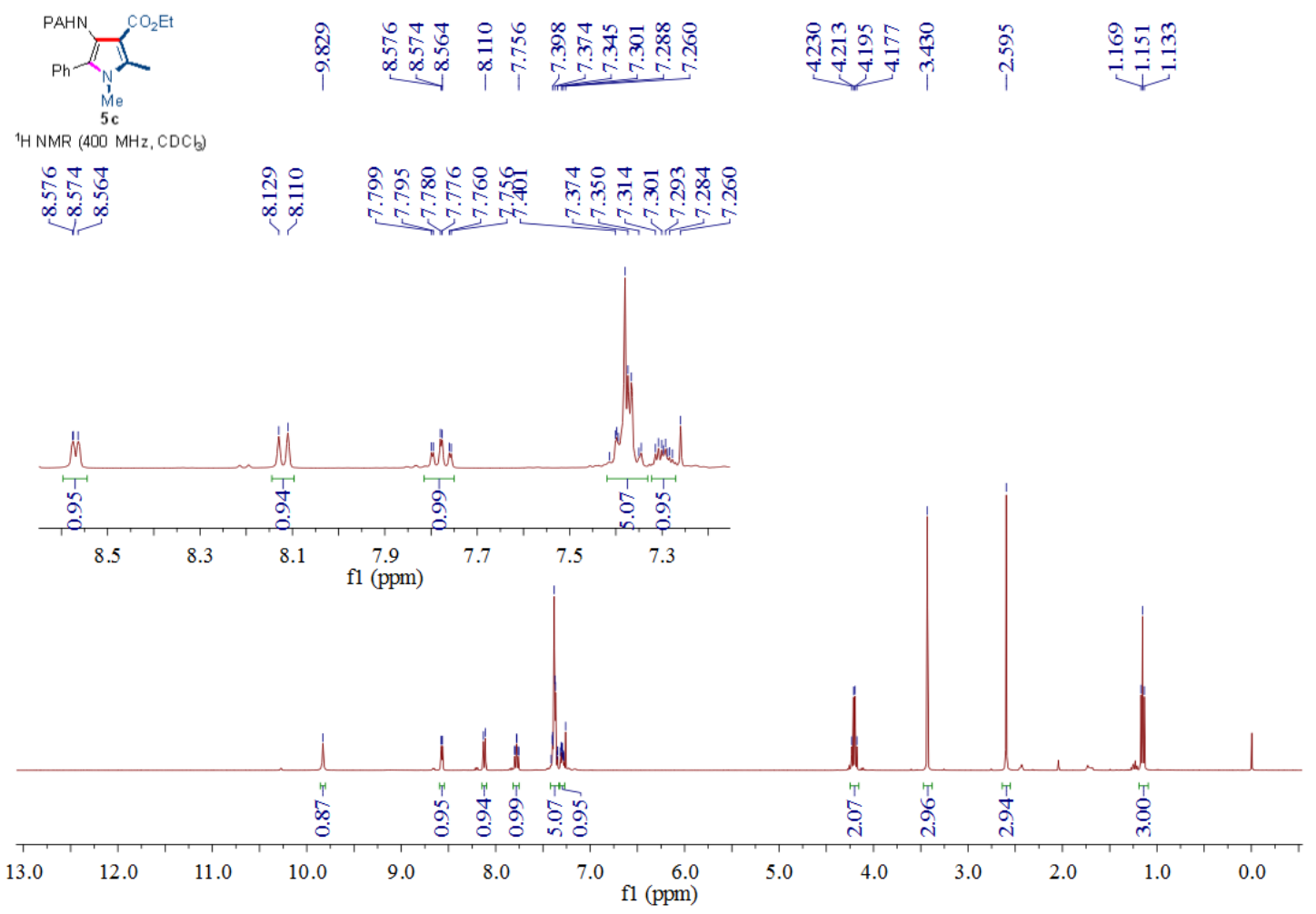


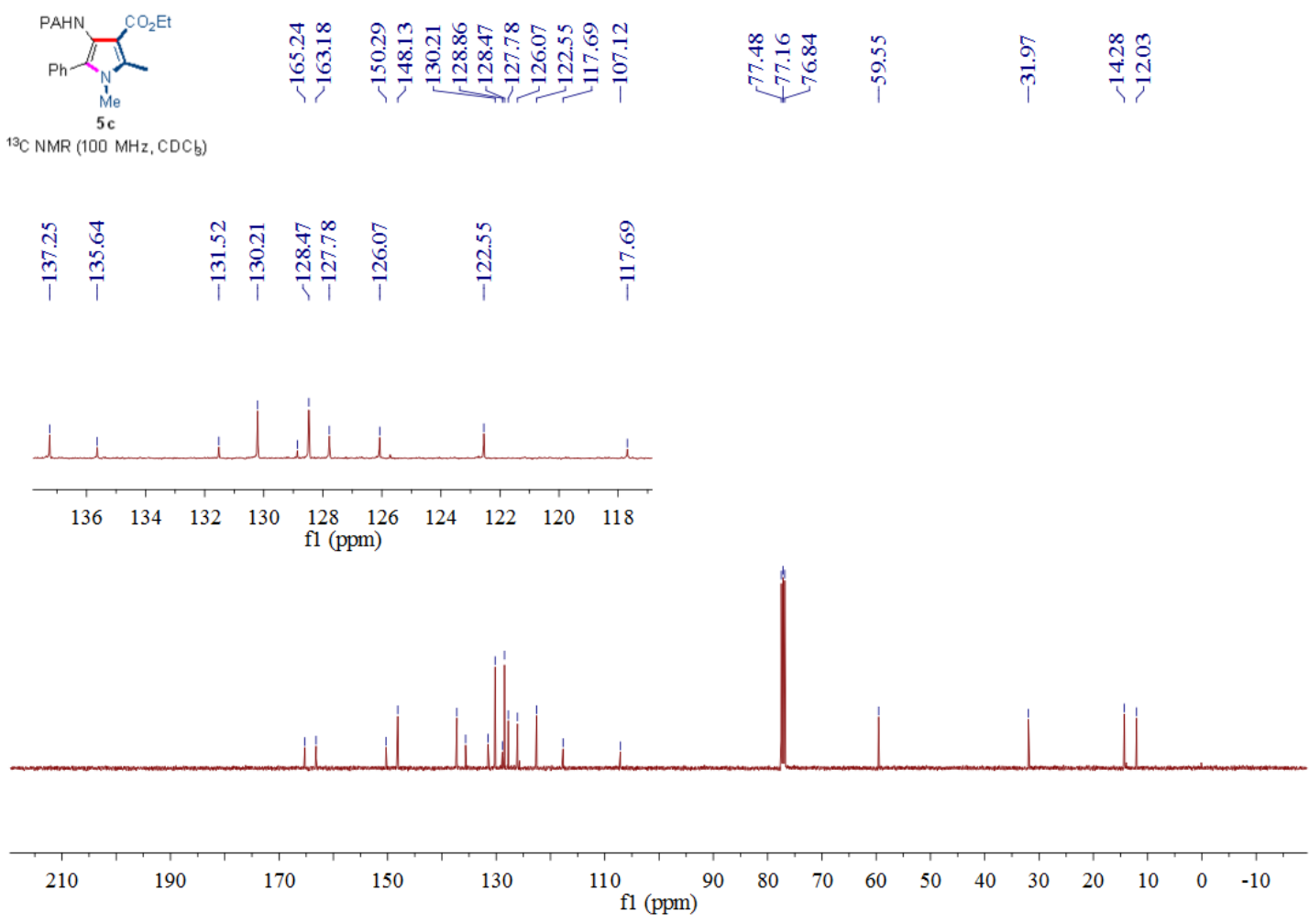

${ }^{1} \mathrm{H}$ NMR and ${ }^{13} \mathrm{C}$ NMR spectra for compound $\mathbf{5 c}$ (using $\mathrm{CDCl}_{3}$ as solvent)
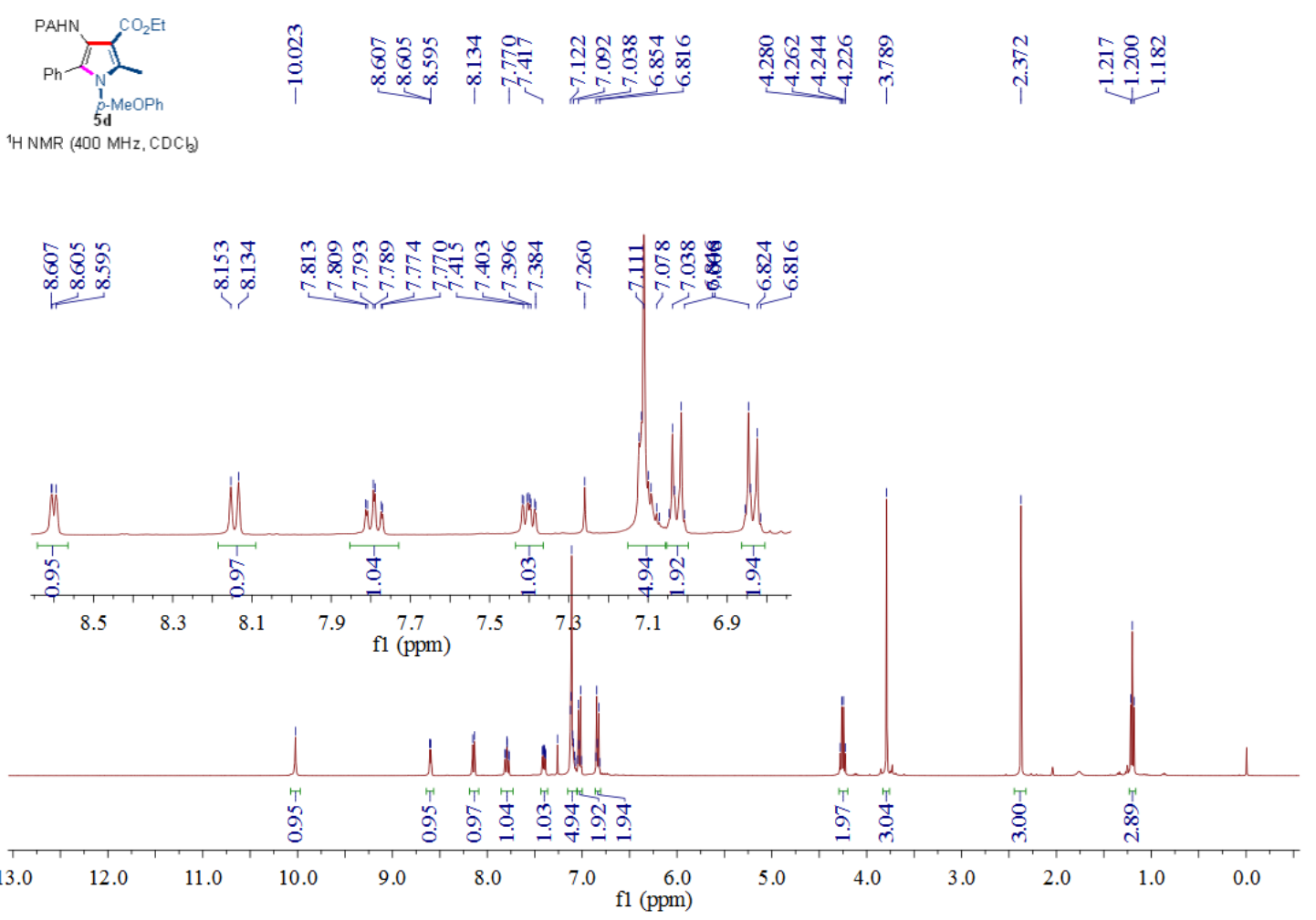


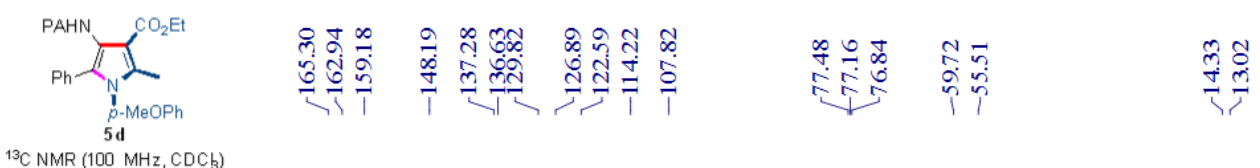

${ }^{13} \mathrm{C}$ NMR $(100 \mathrm{MHz}, \mathrm{CDC} b)$

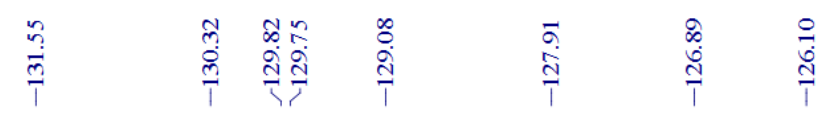
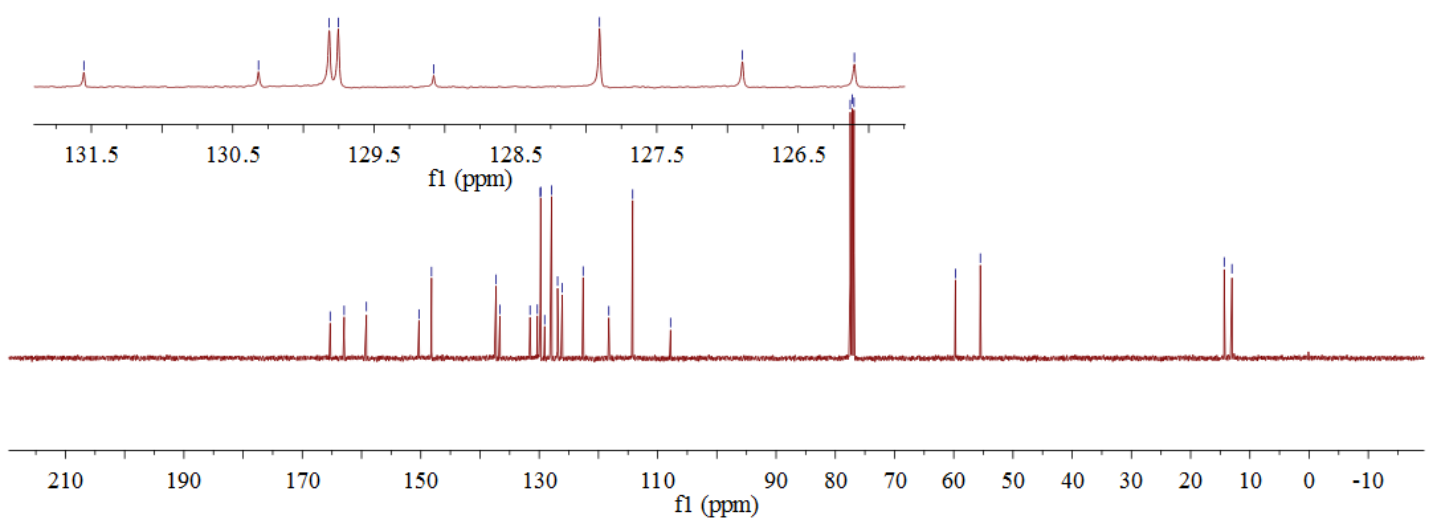

${ }^{1} \mathrm{H}$ NMR and ${ }^{13} \mathrm{C}$ NMR spectra for compound $5 \mathbf{d}$ (using $\mathrm{CDCl}_{3}$ as solvent)

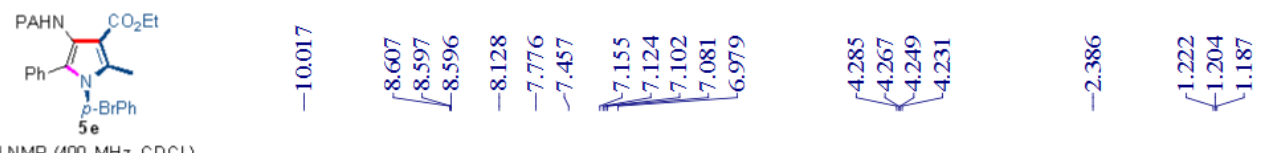

${ }^{1} \mathrm{H}$ NMR $(400 \mathrm{MHz}, \mathrm{CDCb})$

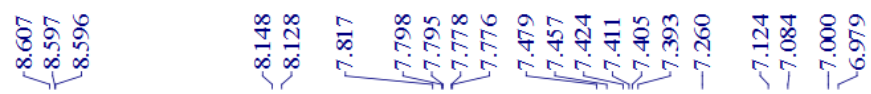
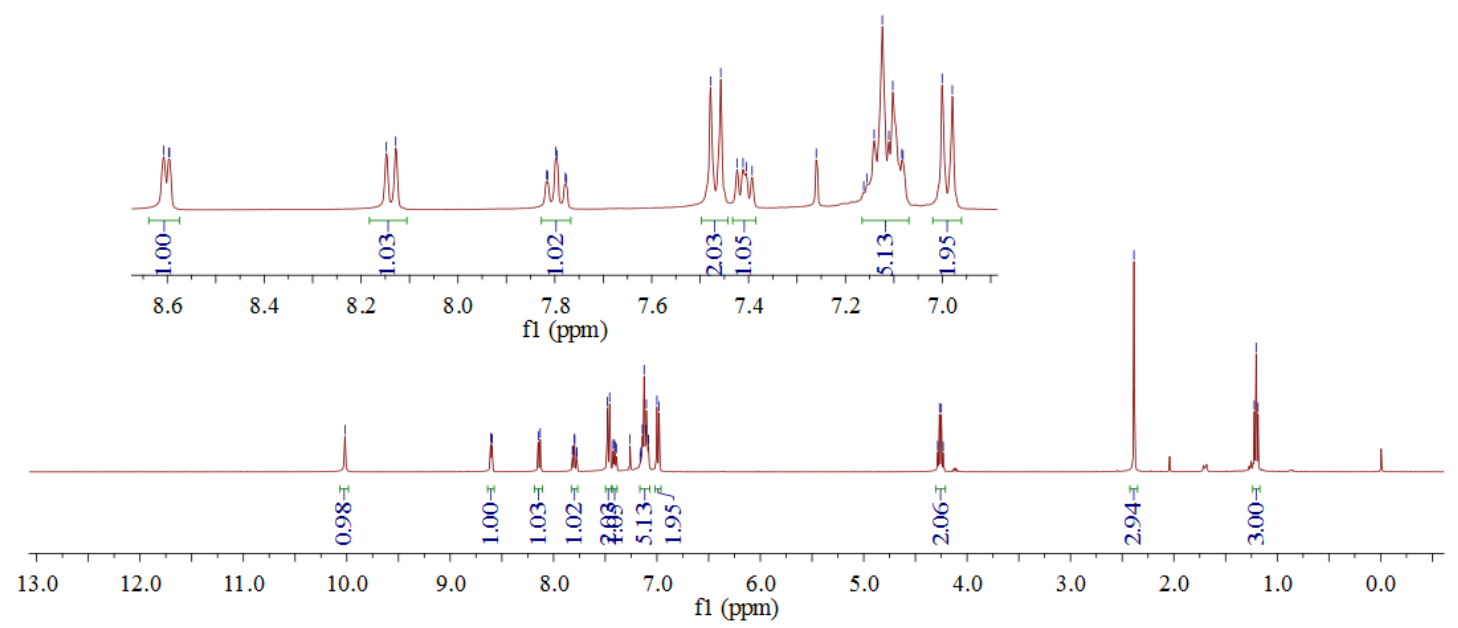


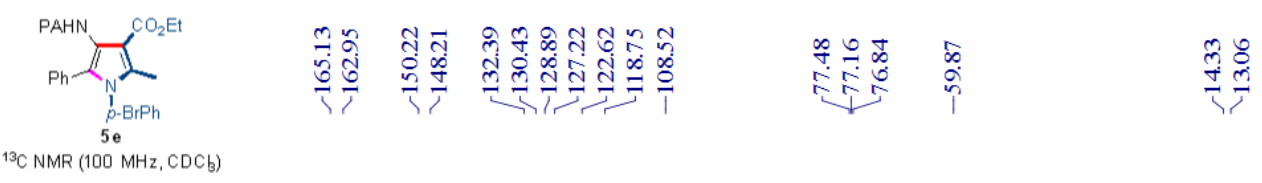

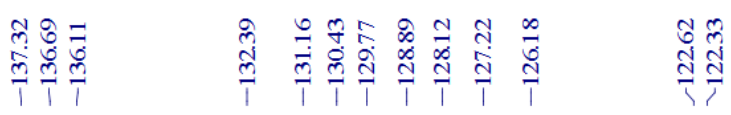
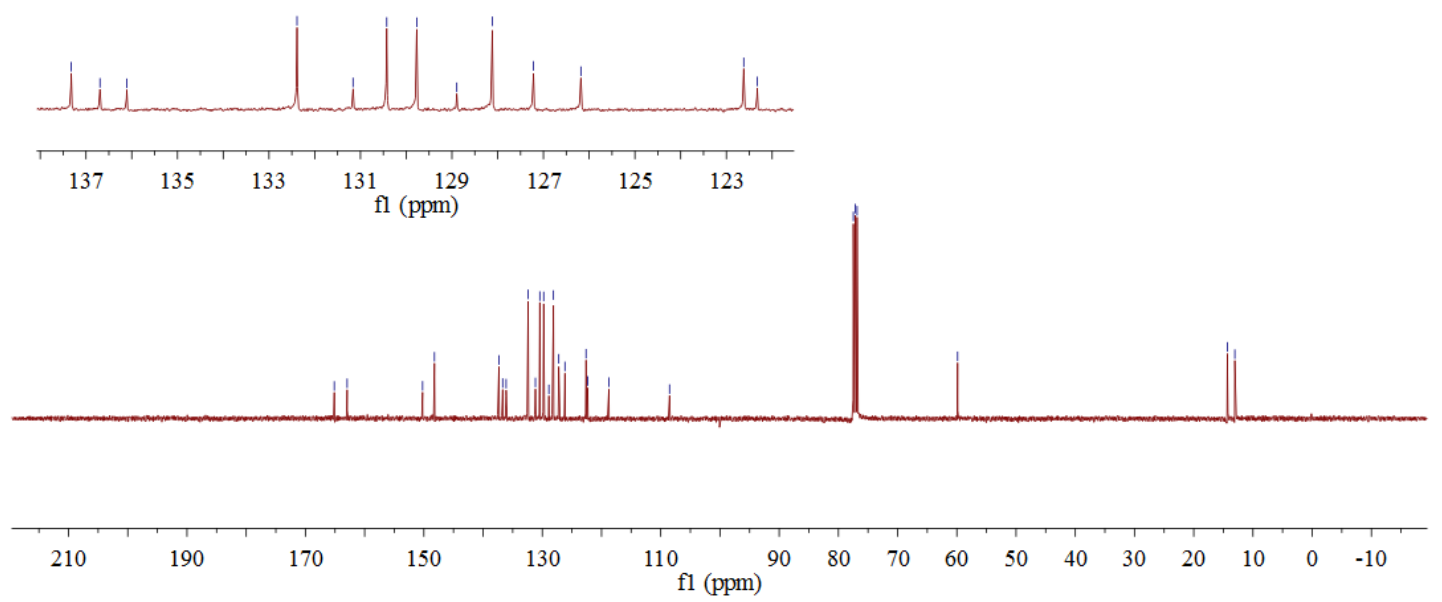

${ }^{1} \mathrm{H}$ NMR and ${ }^{13} \mathrm{C}$ NMR spectra for compound $5 \mathbf{e}$ (using $\mathrm{CDCl}_{3}$ as solvent)
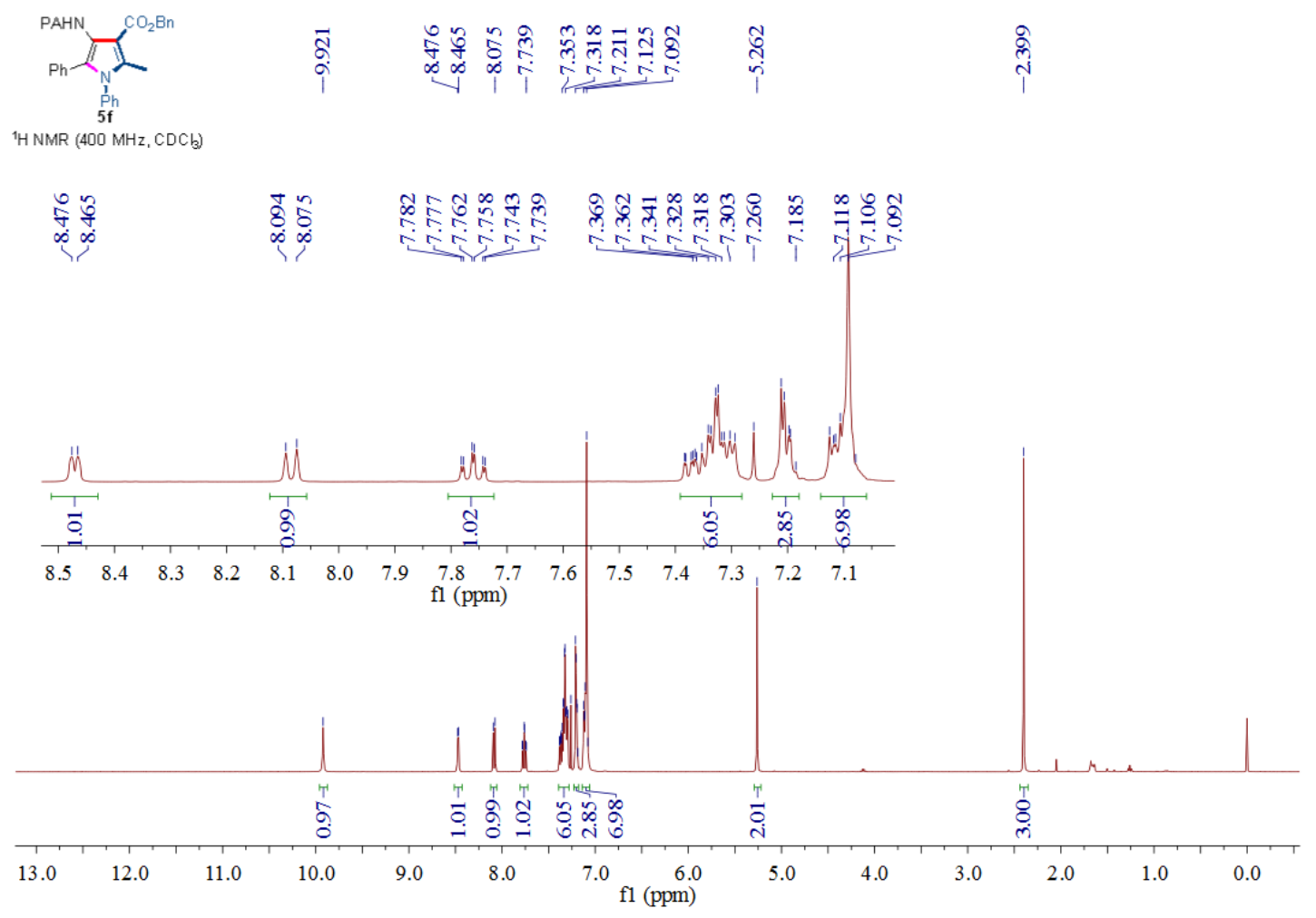


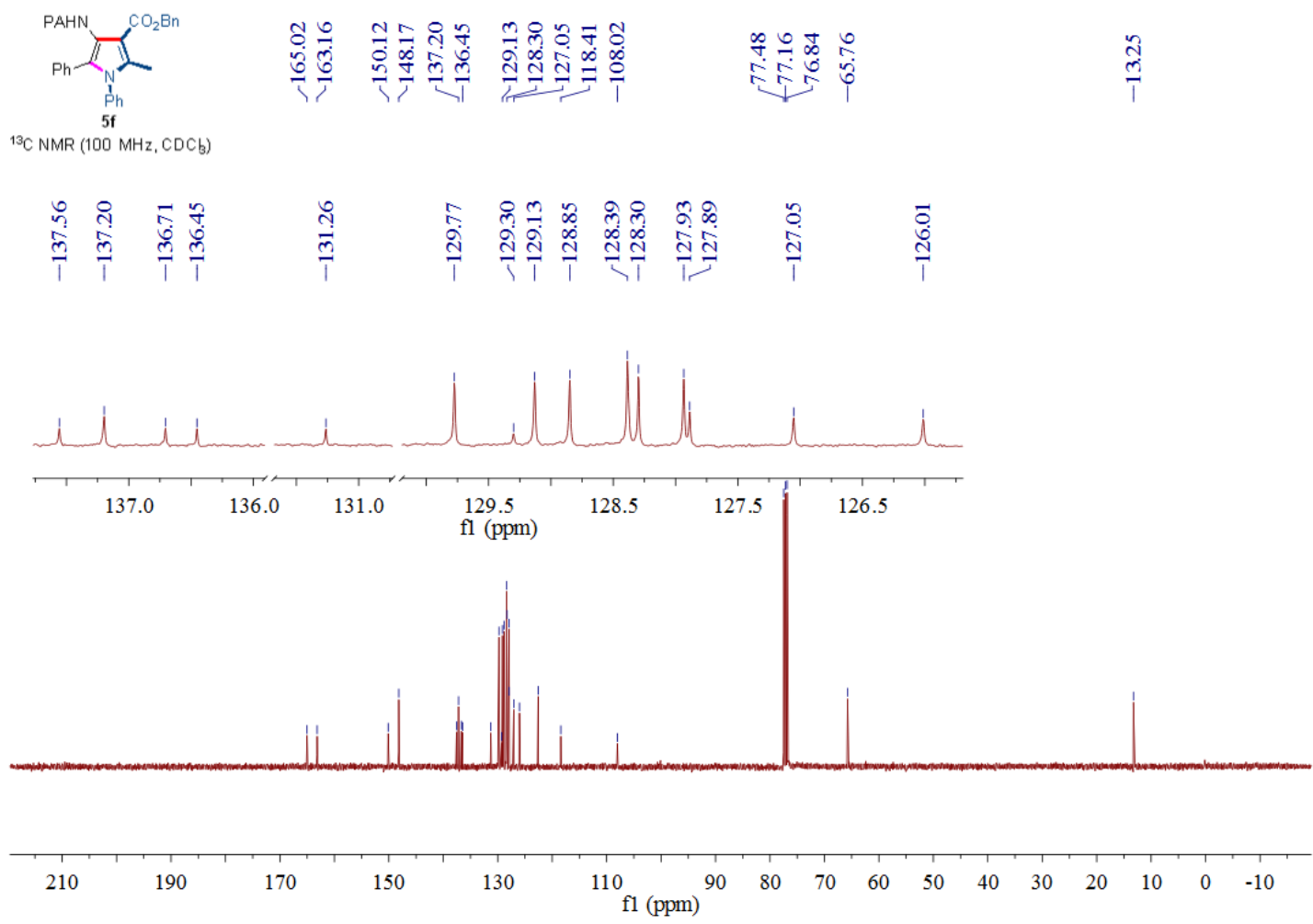

${ }^{1} \mathrm{H}$ NMR and ${ }^{13} \mathrm{C}$ NMR spectra for compound $\mathbf{5 f}$ (using $\mathrm{CDCl}_{3}$ as solvent)

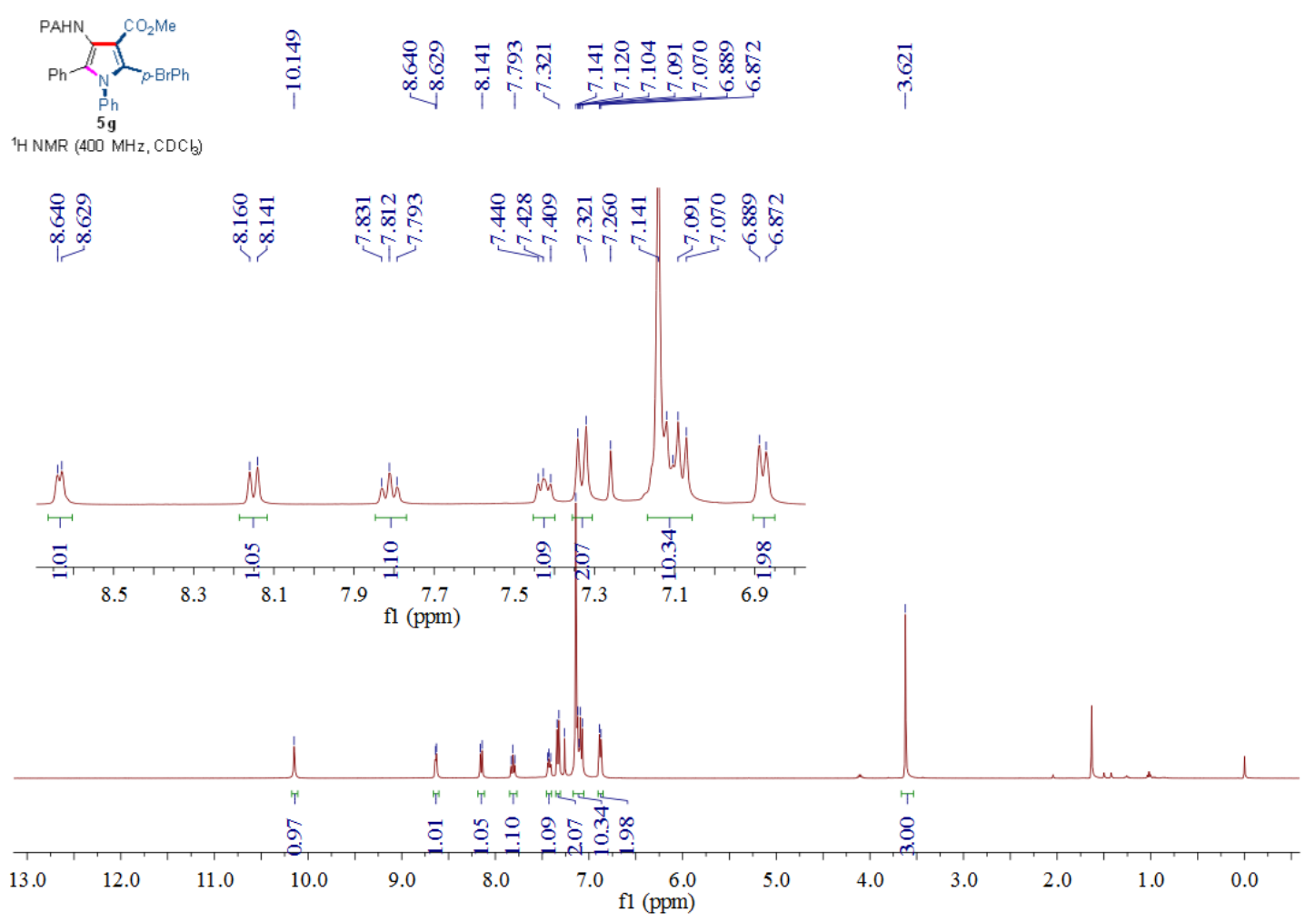



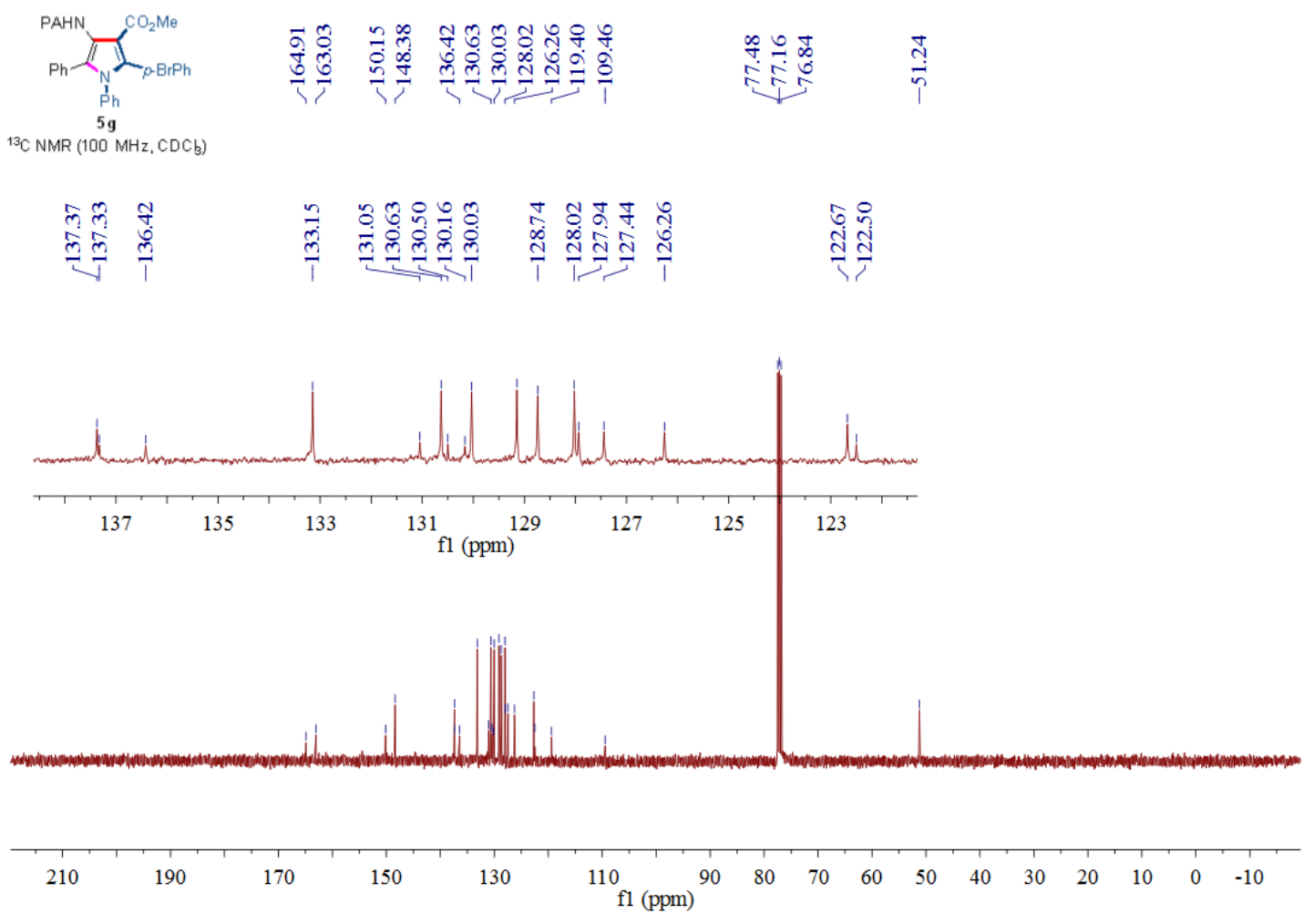

${ }^{1} \mathrm{H}$ NMR and ${ }^{13} \mathrm{C}$ NMR spectra for compound $\mathbf{5 g}$ (using $\mathrm{CDCl}_{3}$ as solvent)
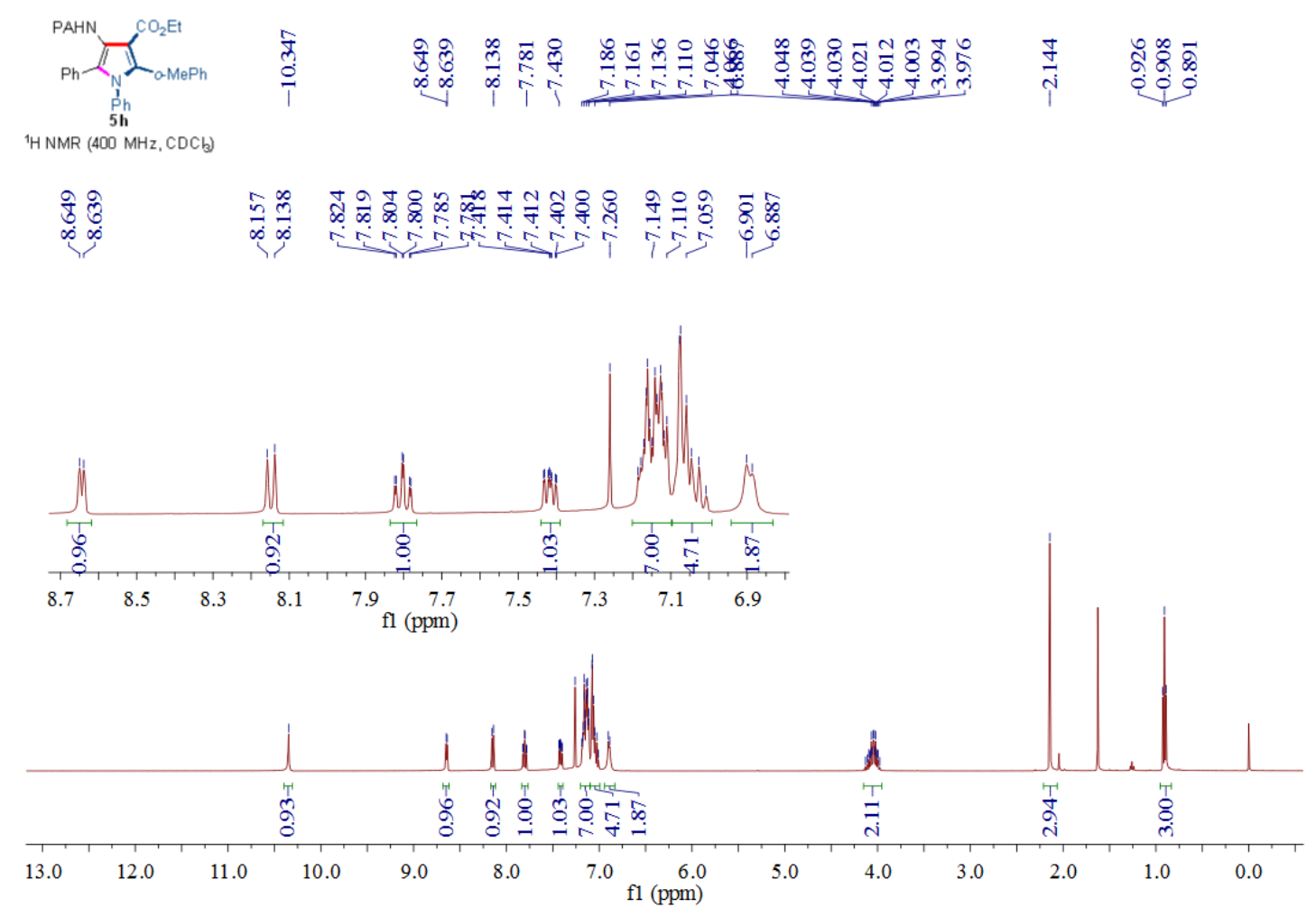

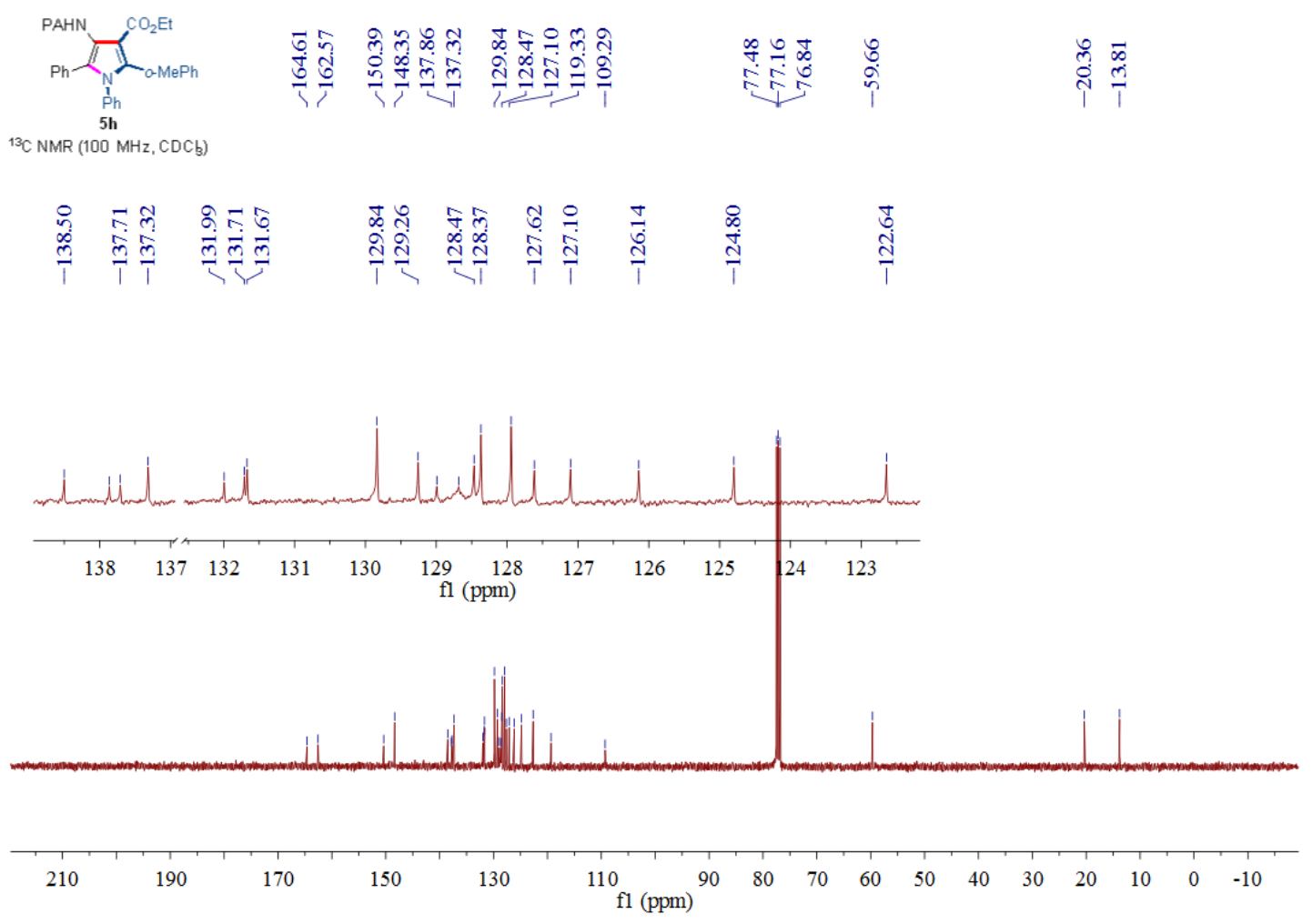

${ }^{1} \mathrm{H}$ NMR and ${ }^{13} \mathrm{C}$ NMR spectra for compound $5 \mathbf{h}$ (using $\mathrm{CDCl}_{3}$ as solvent)
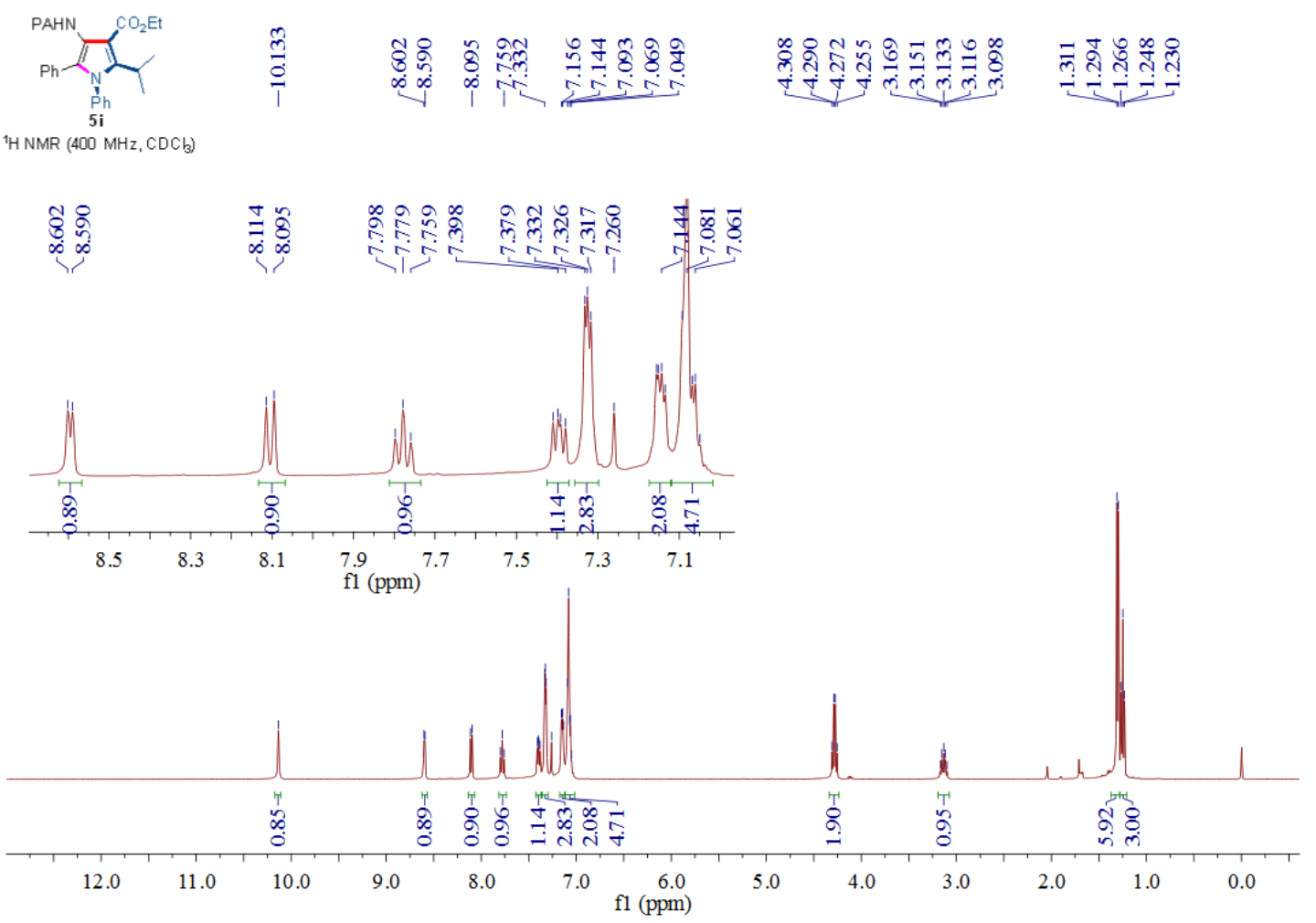

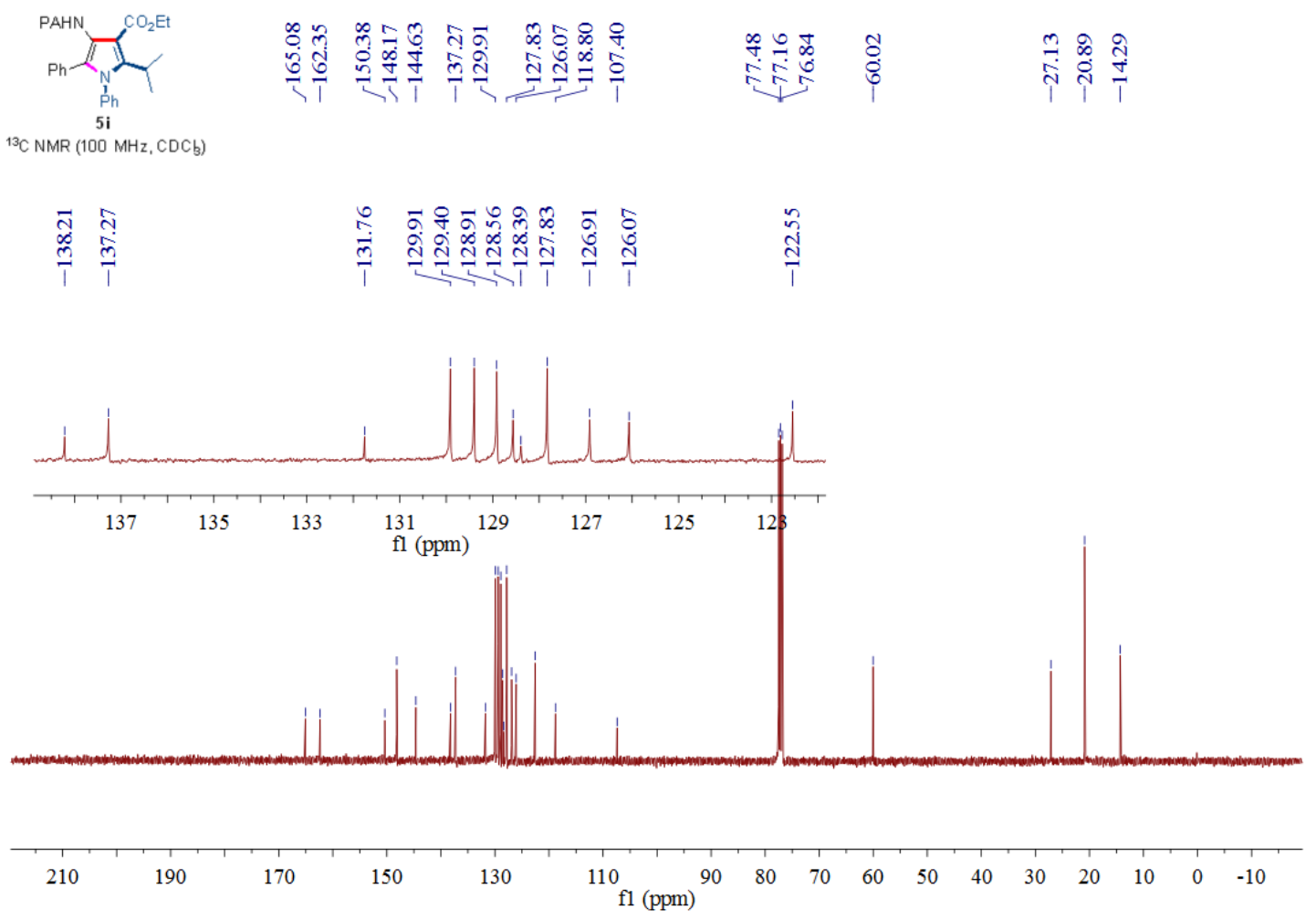

${ }^{1} \mathrm{H}$ NMR and ${ }^{13} \mathrm{C}$ NMR spectra for compound $\mathbf{5 i}$ (using $\mathrm{CDCl}_{3}$ as solvent)
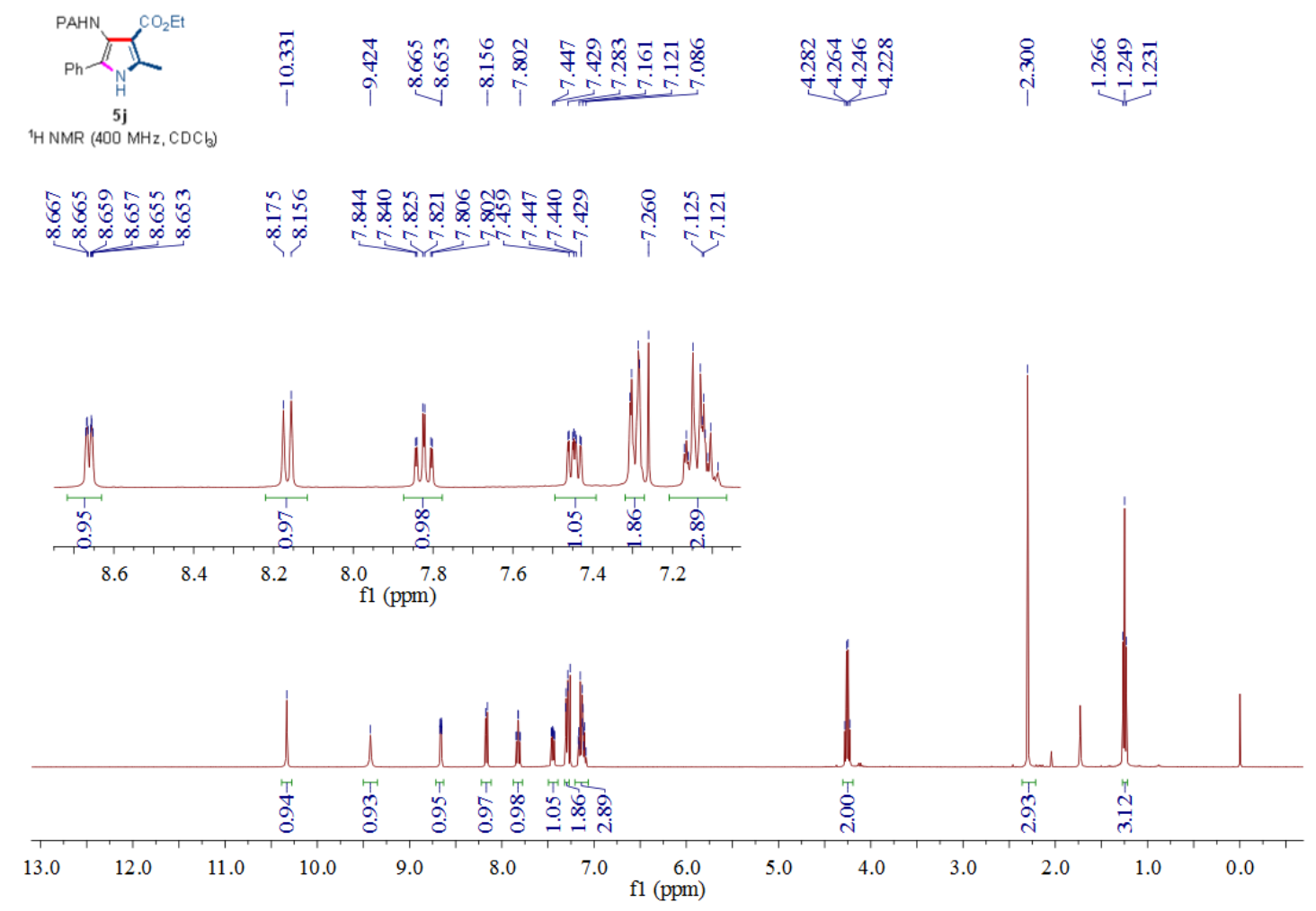


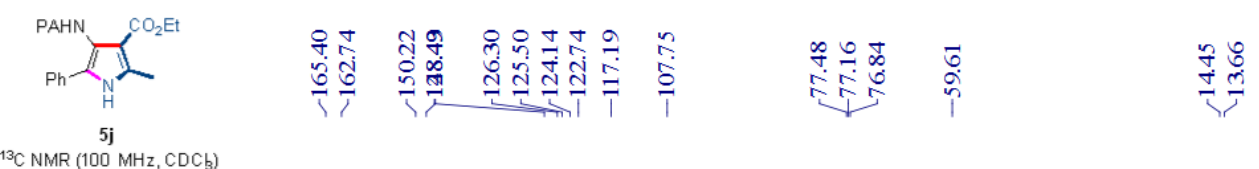

${ }^{13} \mathrm{C}$ NMR $(100 \mathrm{MHz}, \mathrm{CDC} b)$
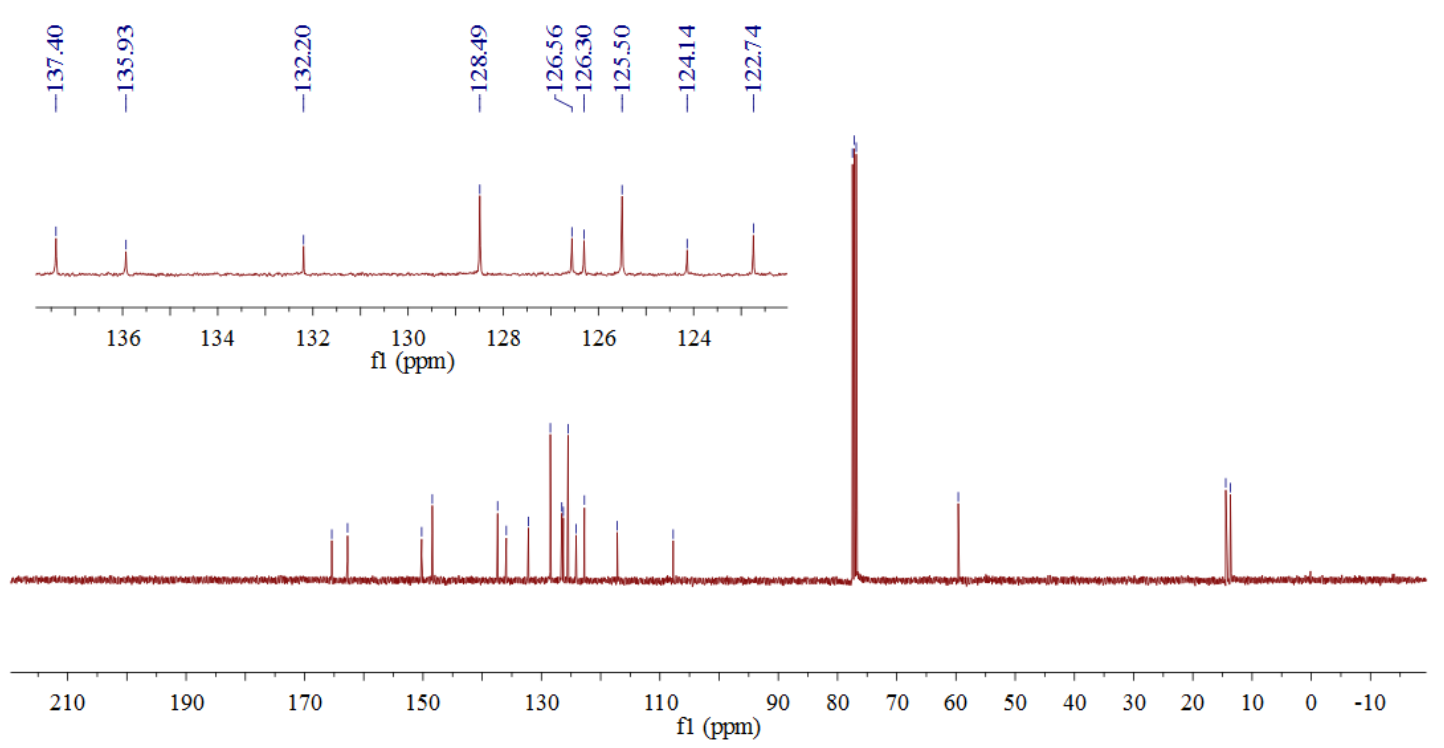

${ }^{1} \mathrm{H}$ NMR and ${ }^{13} \mathrm{C}$ NMR spectra for compound $\mathbf{5} \mathbf{j}$ (using $\mathrm{CDCl}_{3}$ as solvent)
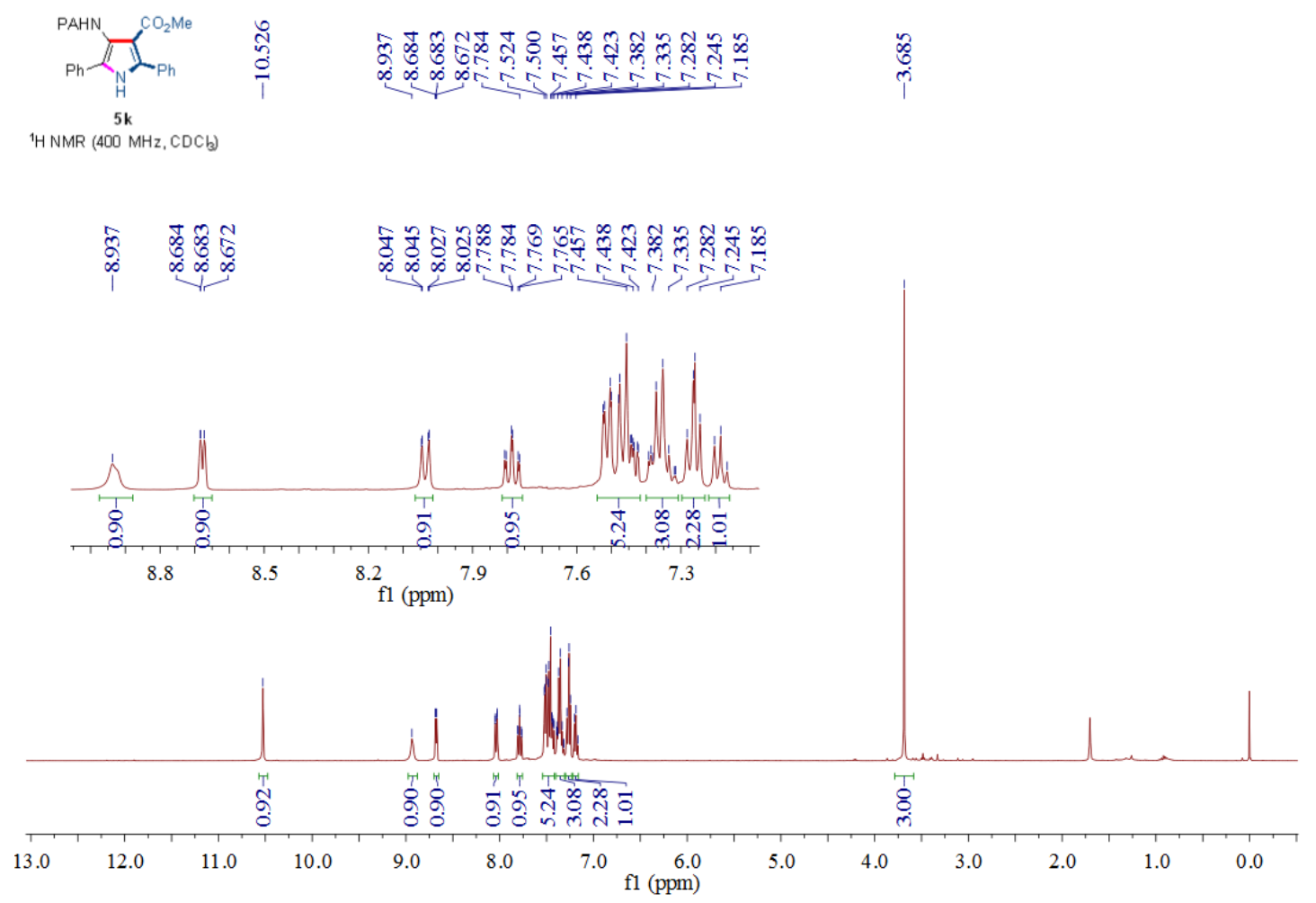


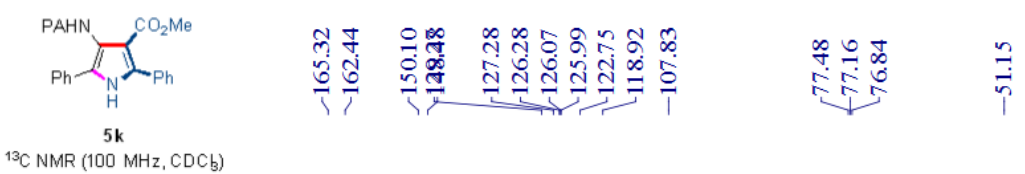

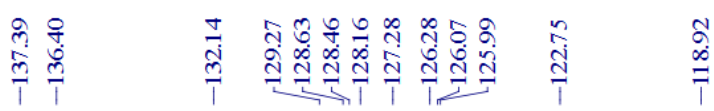
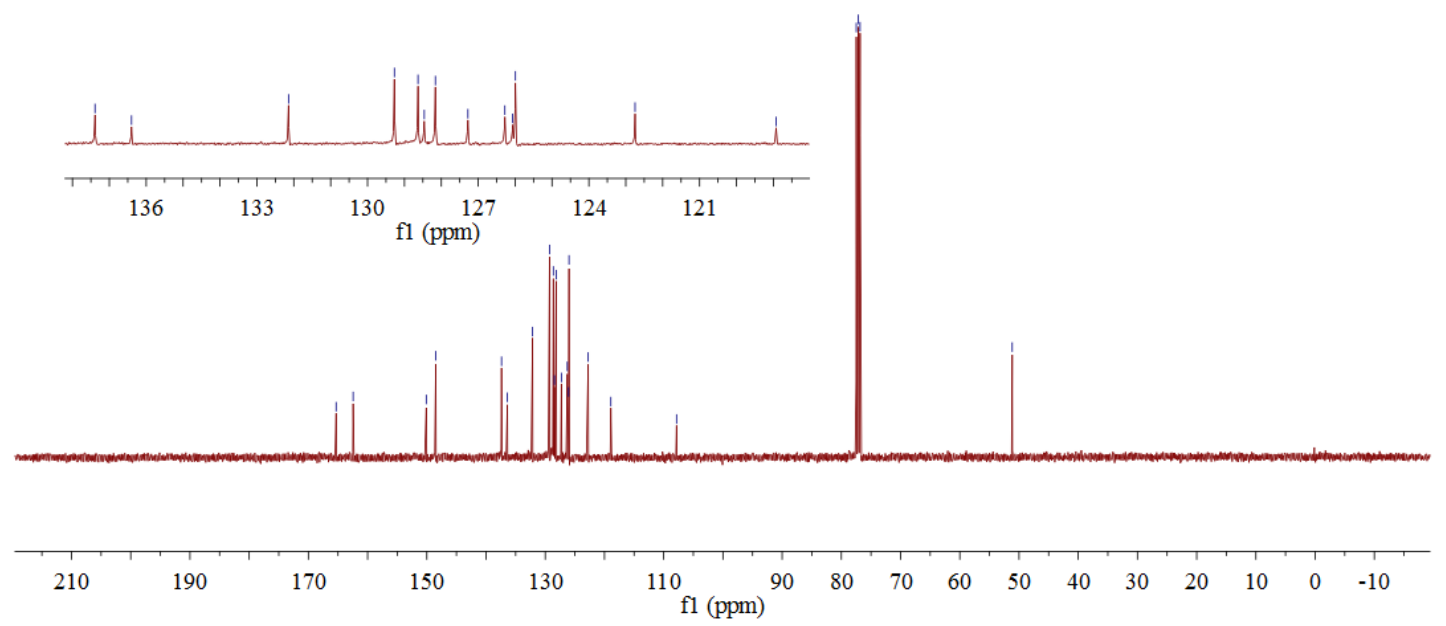

${ }^{1} \mathrm{H}$ NMR and ${ }^{13} \mathrm{C}$ NMR spectra for compound 5k (using $\mathrm{CDCl}_{3}$ as solvent)

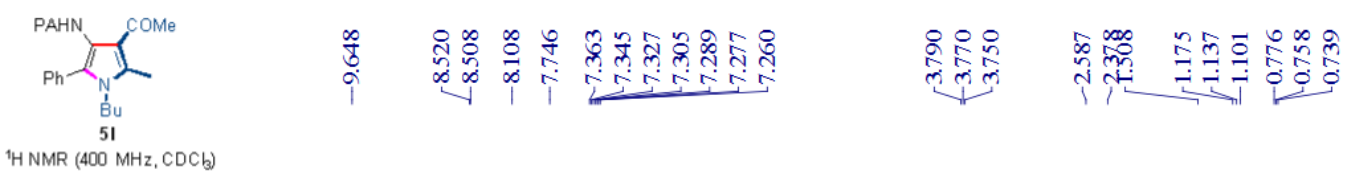

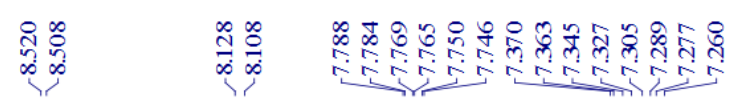

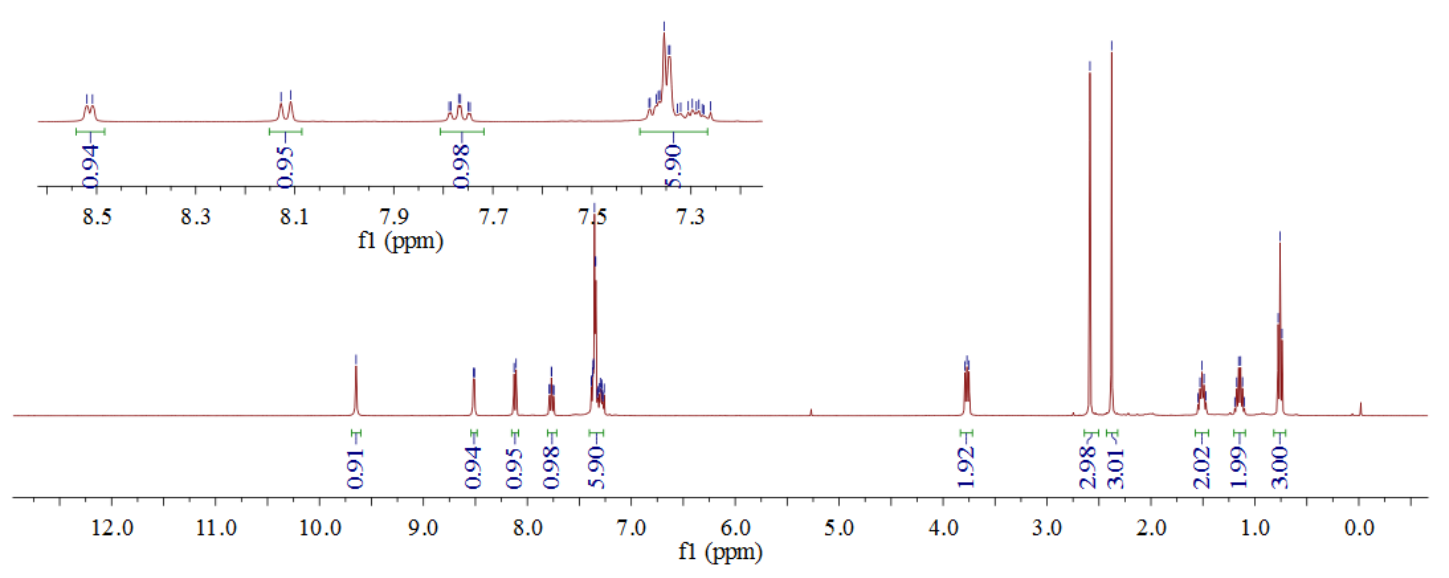




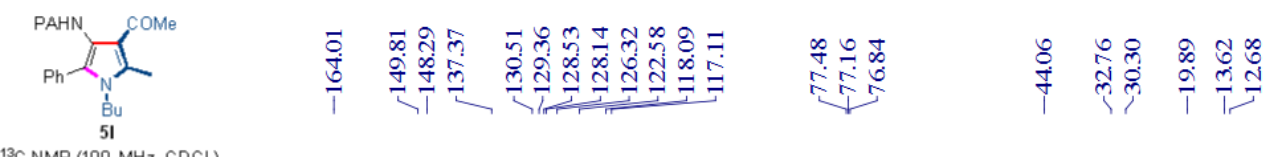

${ }^{13} \mathrm{C}$ NMR $(100 \mathrm{MHz}, \mathrm{CDC}$ )

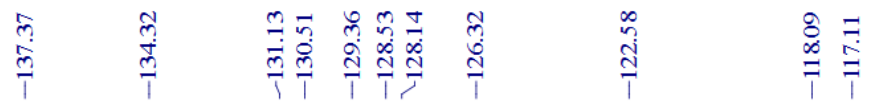
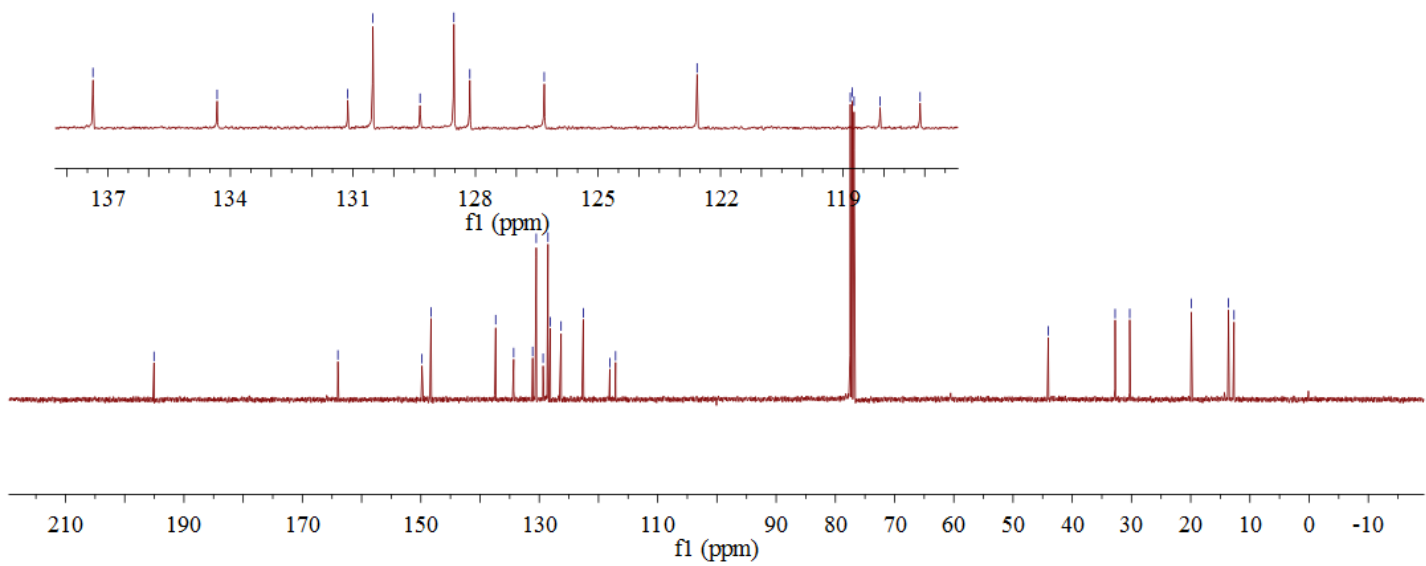

${ }^{1} \mathrm{H}$ NMR and ${ }^{13} \mathrm{C}$ NMR spectra for compound $\mathbf{5 l}$ (using $\mathrm{CDCl}_{3}$ as solvent)

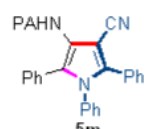

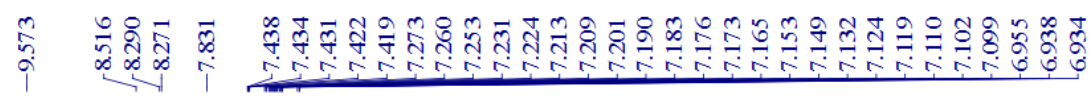

1H NMR (400 MHz, $\mathrm{CDC}$ )

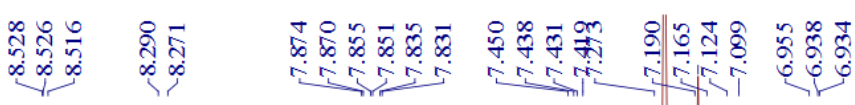

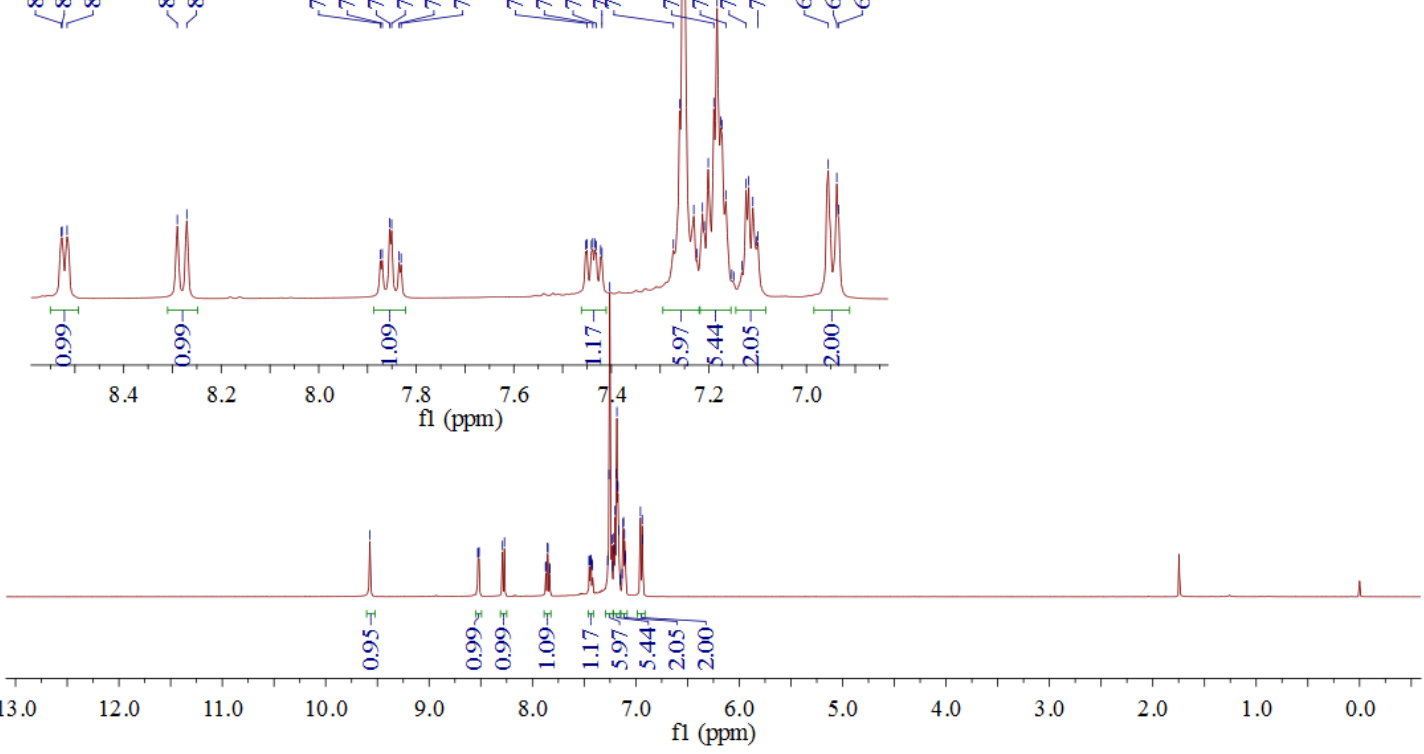




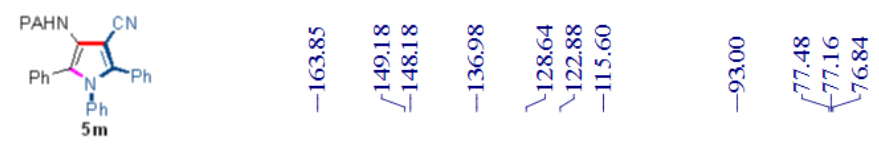

${ }^{13} \mathrm{C}$ NMR $(100 \mathrm{MHz}, \mathrm{CDCb})$

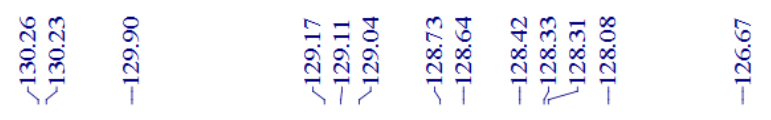
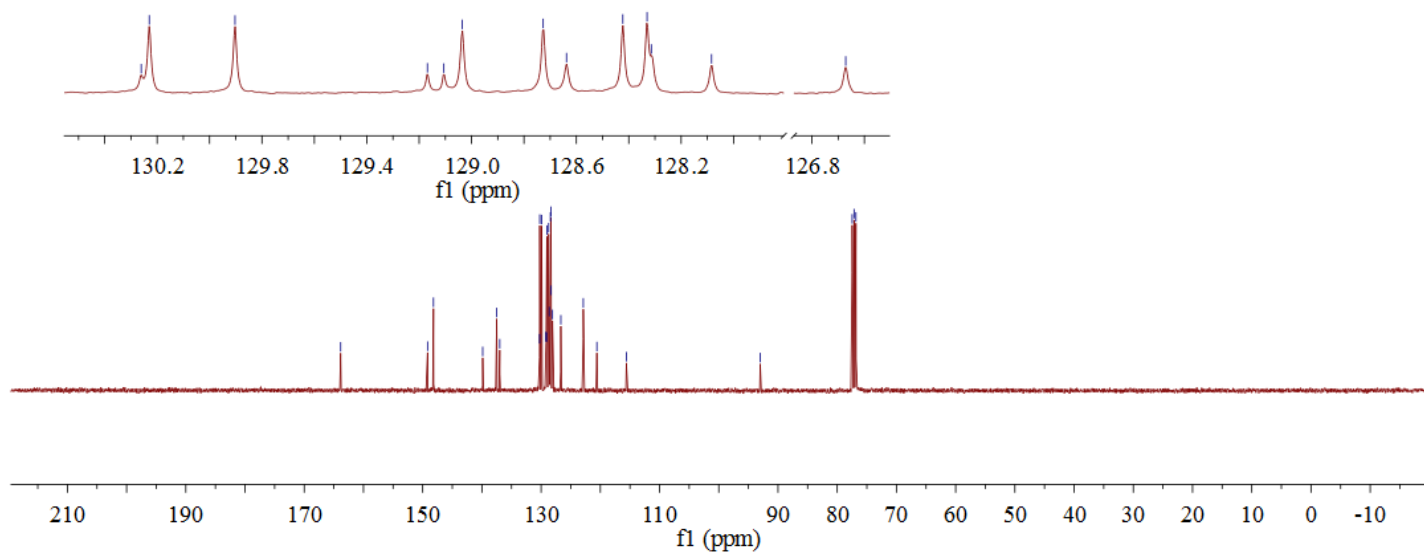

${ }^{1} \mathrm{H}$ NMR and ${ }^{13} \mathrm{C}$ NMR spectra for compound $\mathbf{5 m}$ (using $\mathrm{CDCl}_{3}$ as solvent)

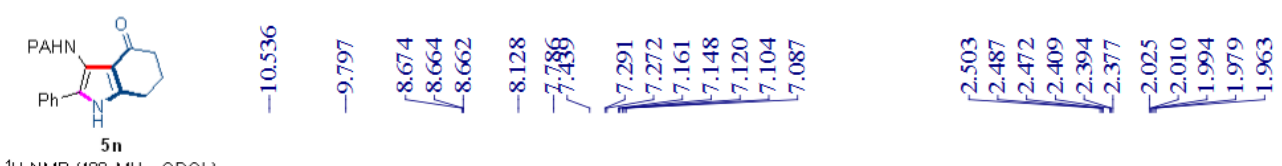

${ }^{1} \mathrm{H}$ NMR $(400 \mathrm{MHz}, \mathrm{CDC}$ )

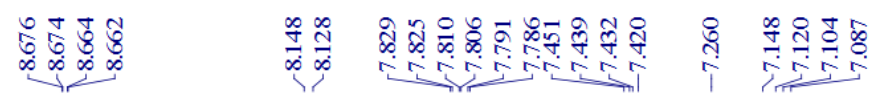
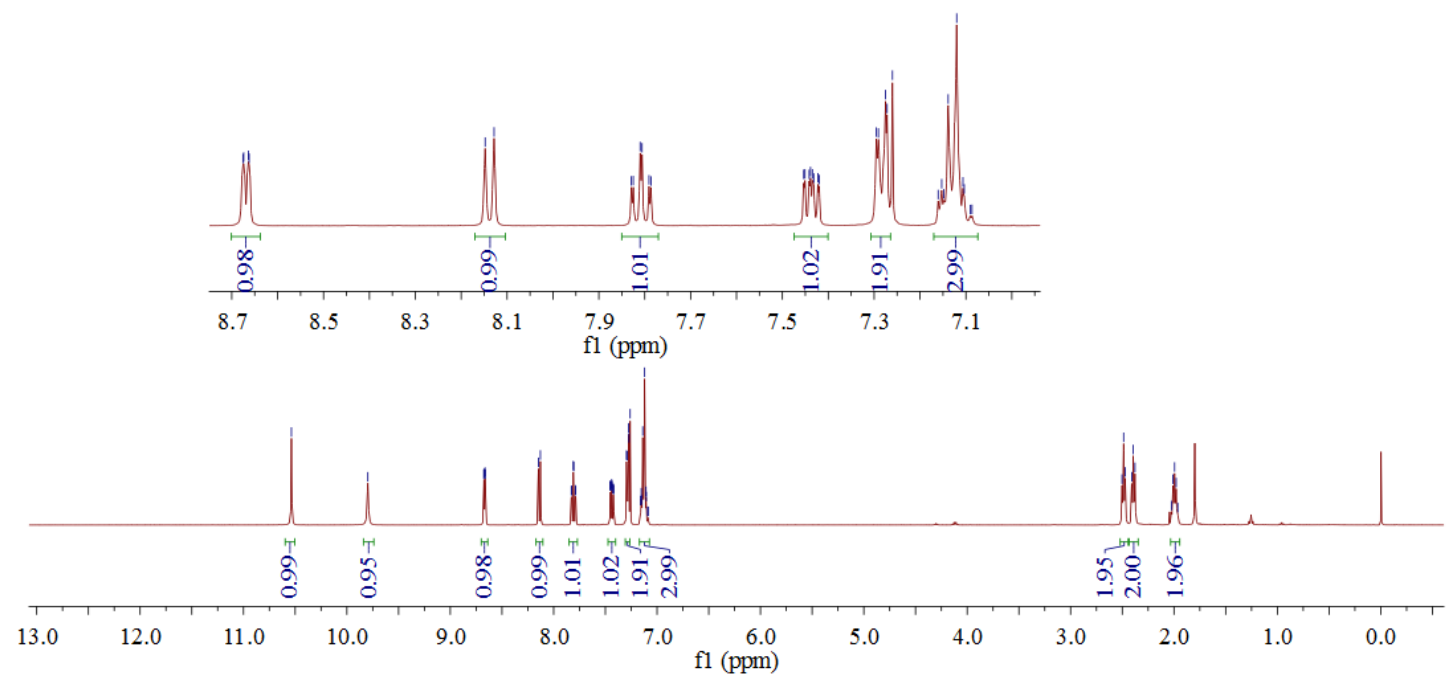

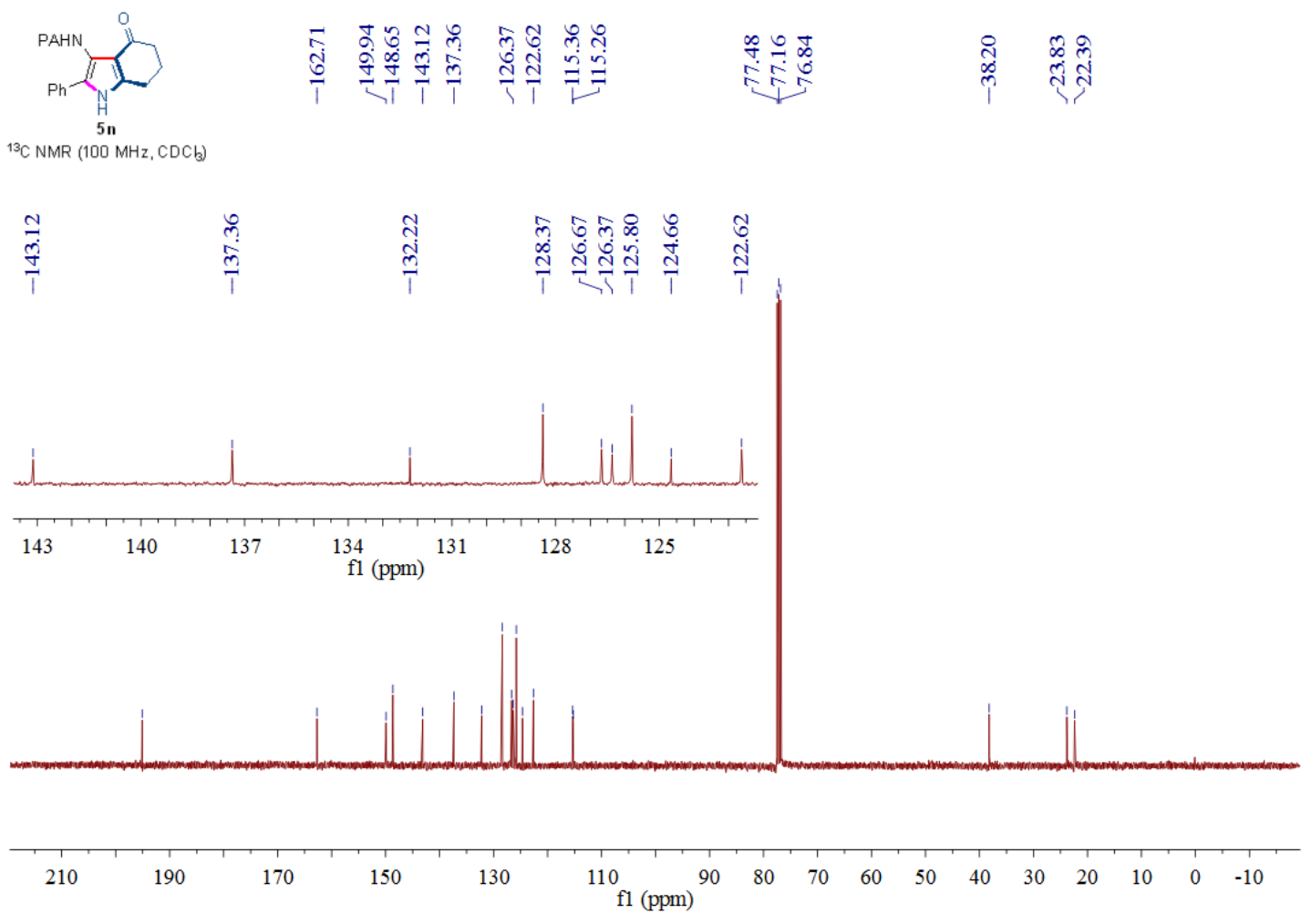

${ }^{1} \mathrm{H}$ NMR and ${ }^{13} \mathrm{C}$ NMR spectra for compound 5n (using $\mathrm{CDCl}_{3}$ as solvent)

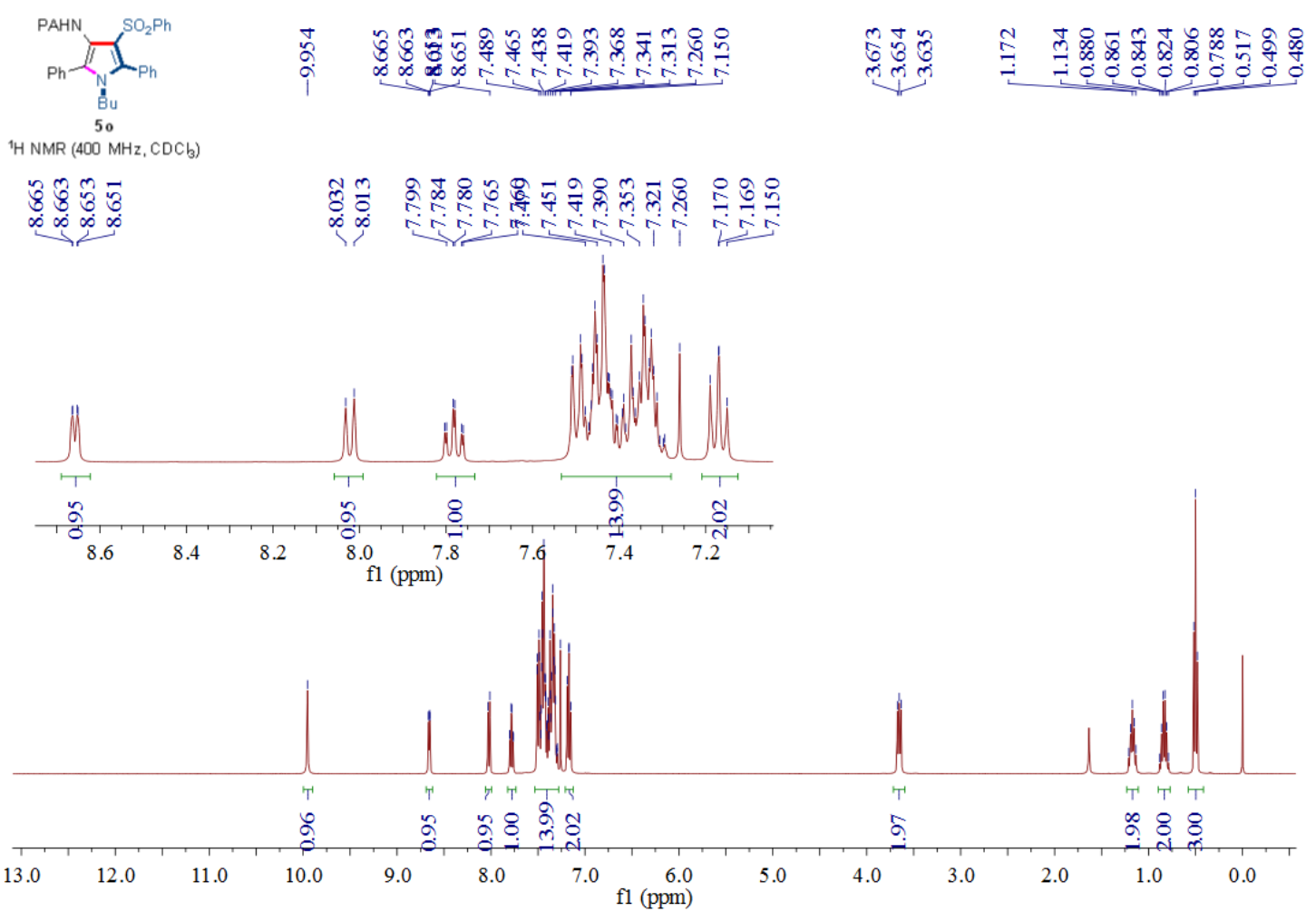




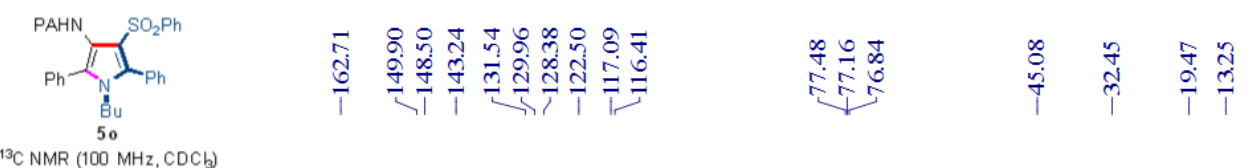

${ }^{13} \mathrm{C}$ NMR $(100 \mathrm{MHz}, \mathrm{CDCb})$

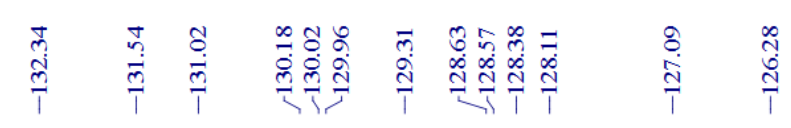
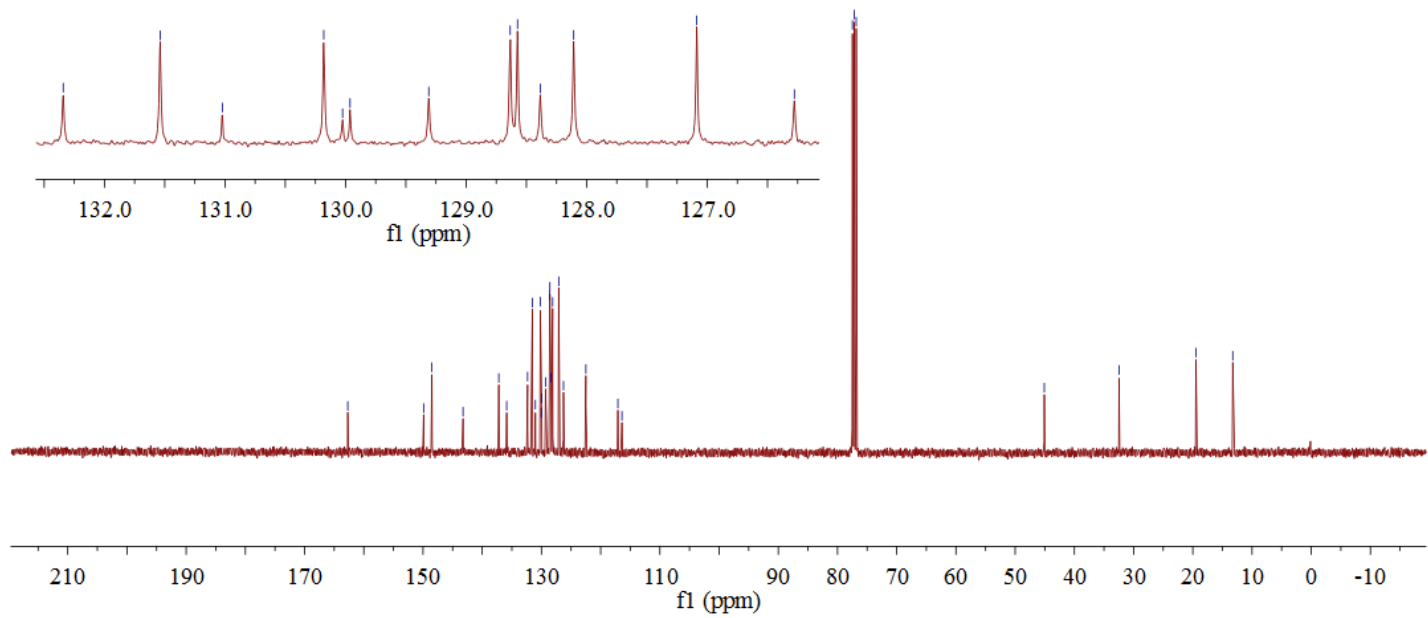

${ }^{1} \mathrm{H}$ NMR and ${ }^{13} \mathrm{C}$ NMR spectra for compound 50 (using $\mathrm{CDCl}_{3}$ as solvent)
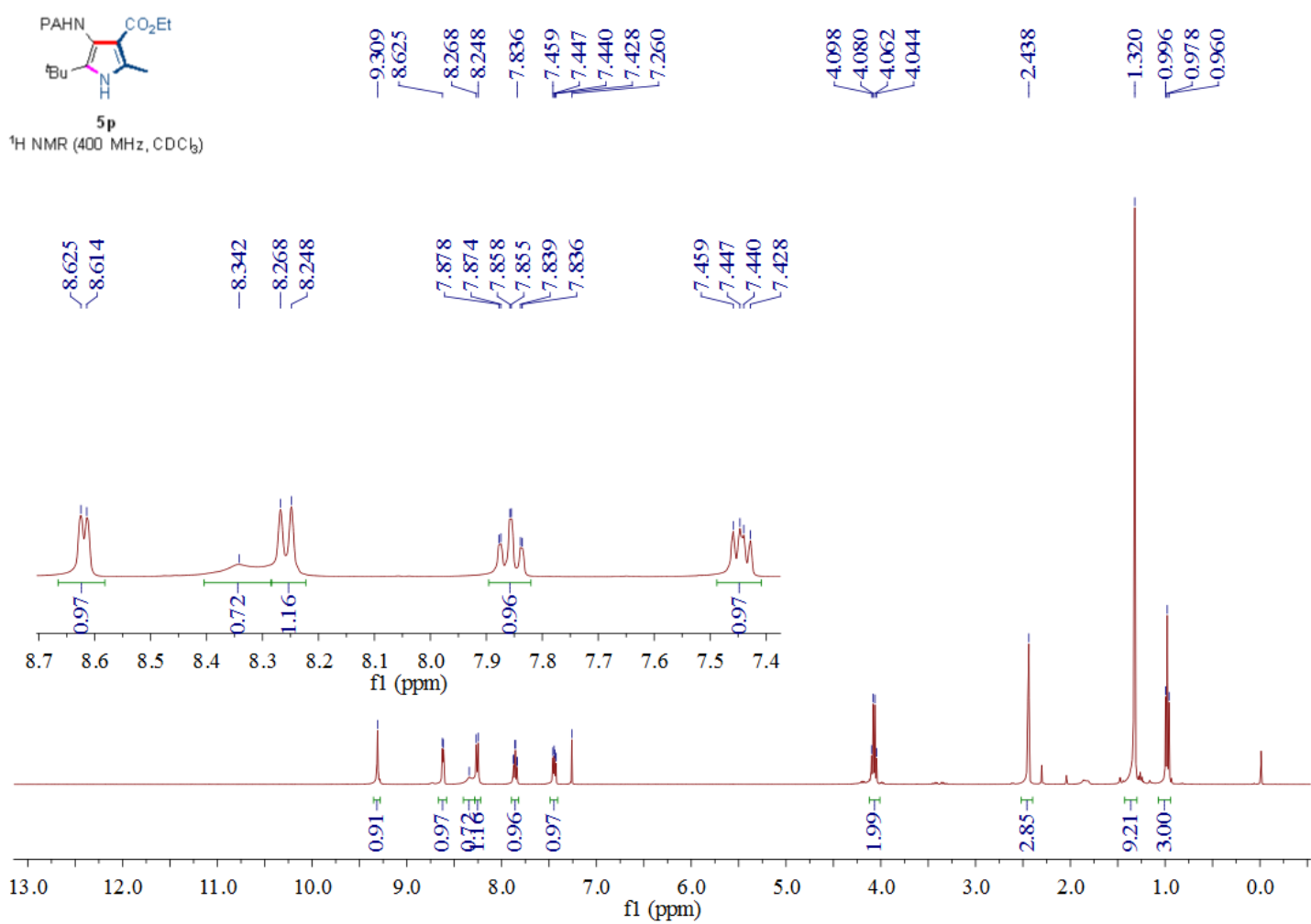

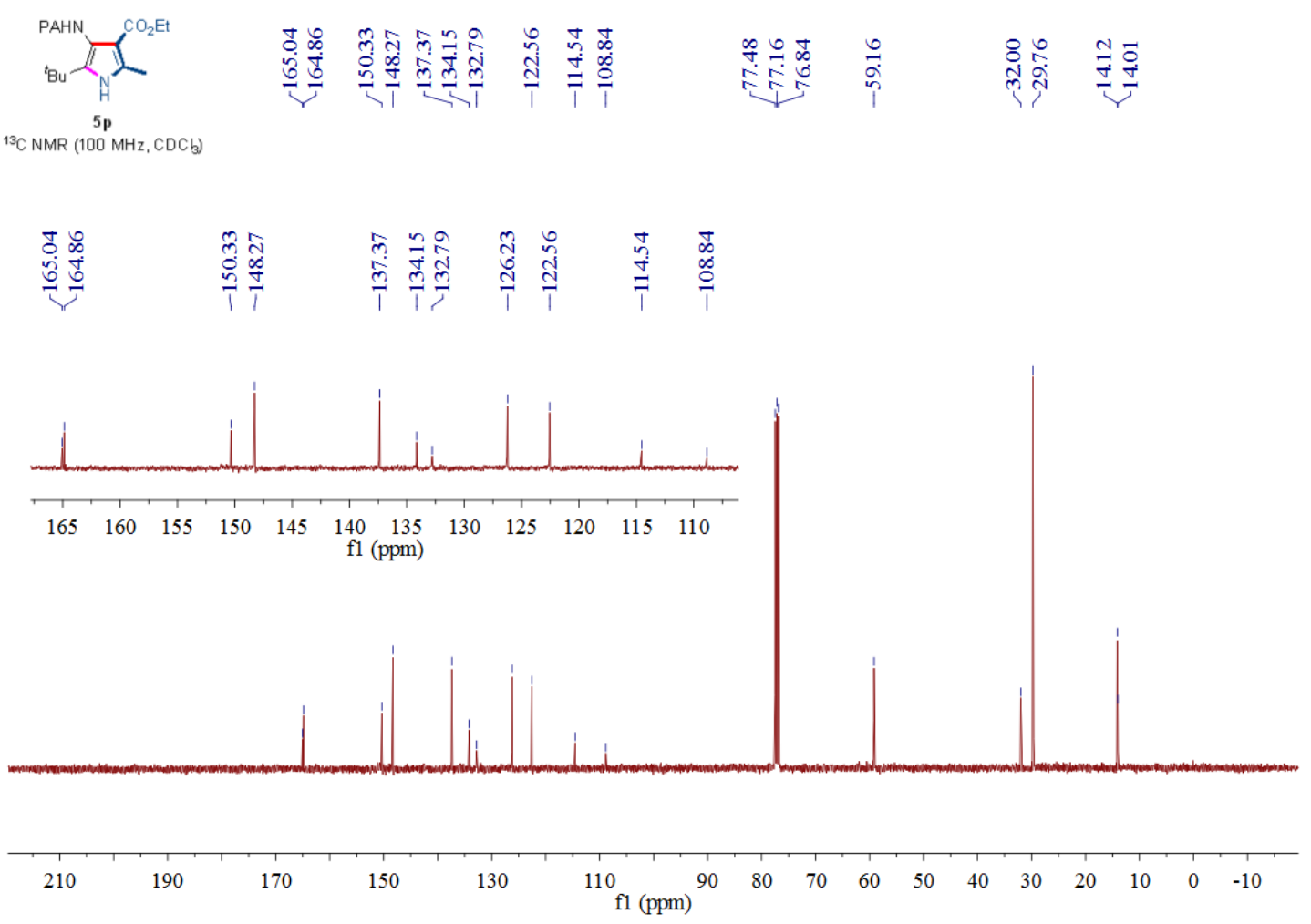

${ }^{1} \mathrm{H}$ NMR and ${ }^{13} \mathrm{C}$ NMR spectra for compound 5p (using $\mathrm{CDCl}_{3}$ as solvent)

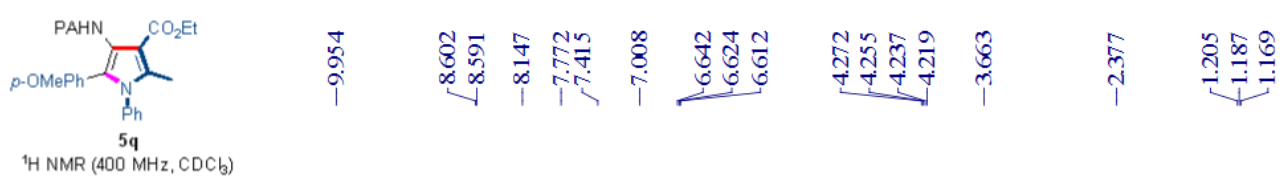

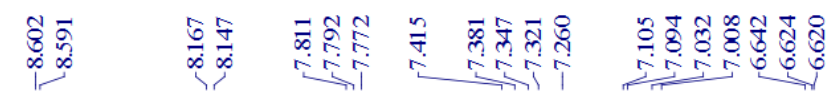
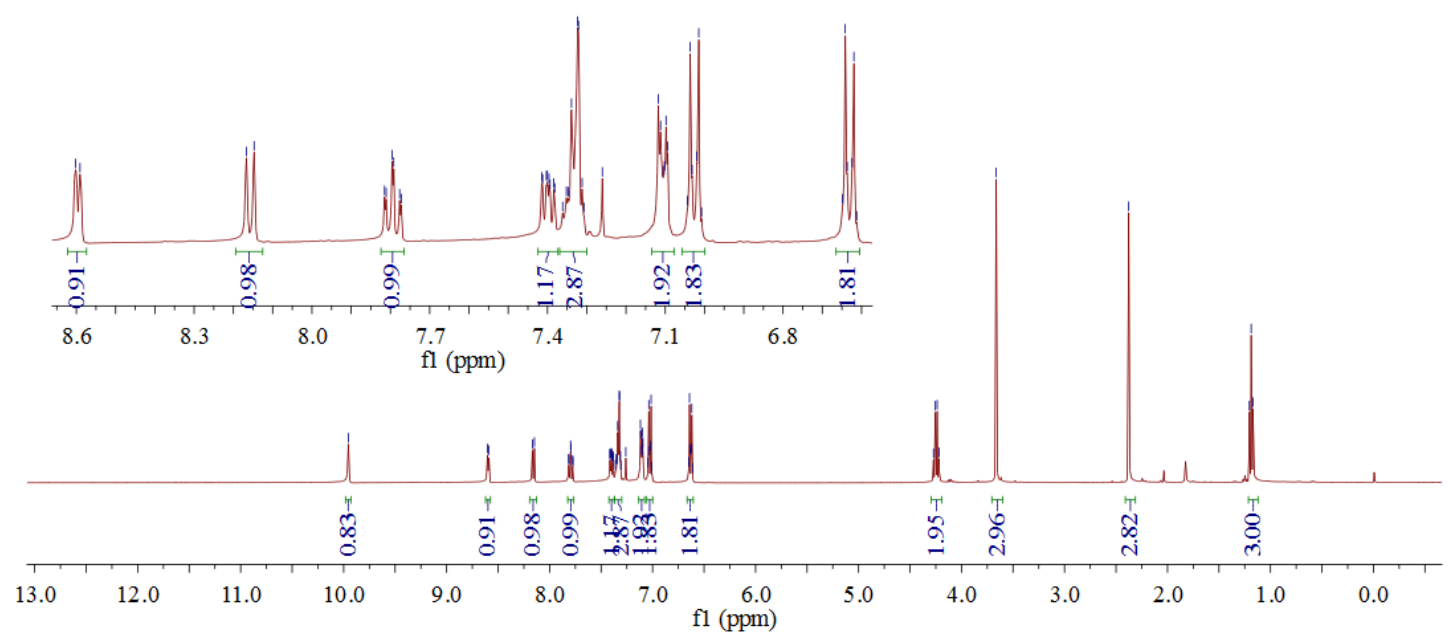

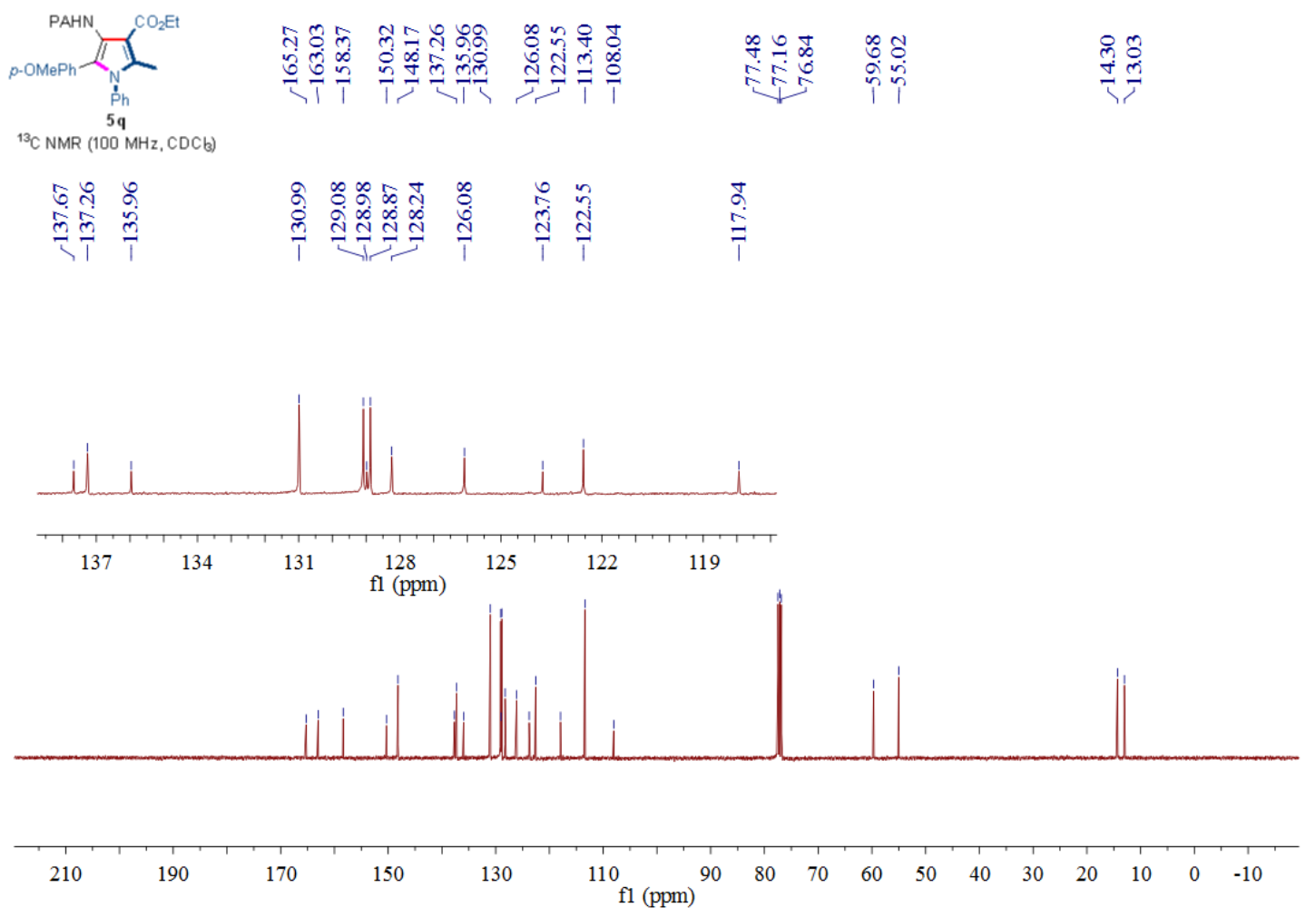

${ }^{1} \mathrm{H}$ NMR and ${ }^{13} \mathrm{C}$ NMR spectra for compound $\mathbf{5 q}$ (using $\mathrm{CDCl}_{3}$ as solvent)

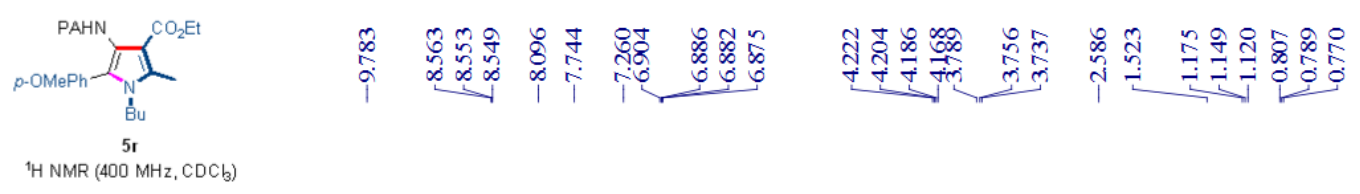

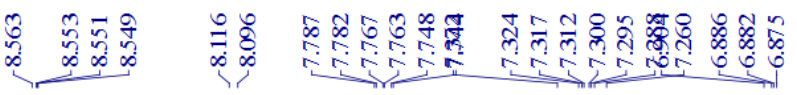

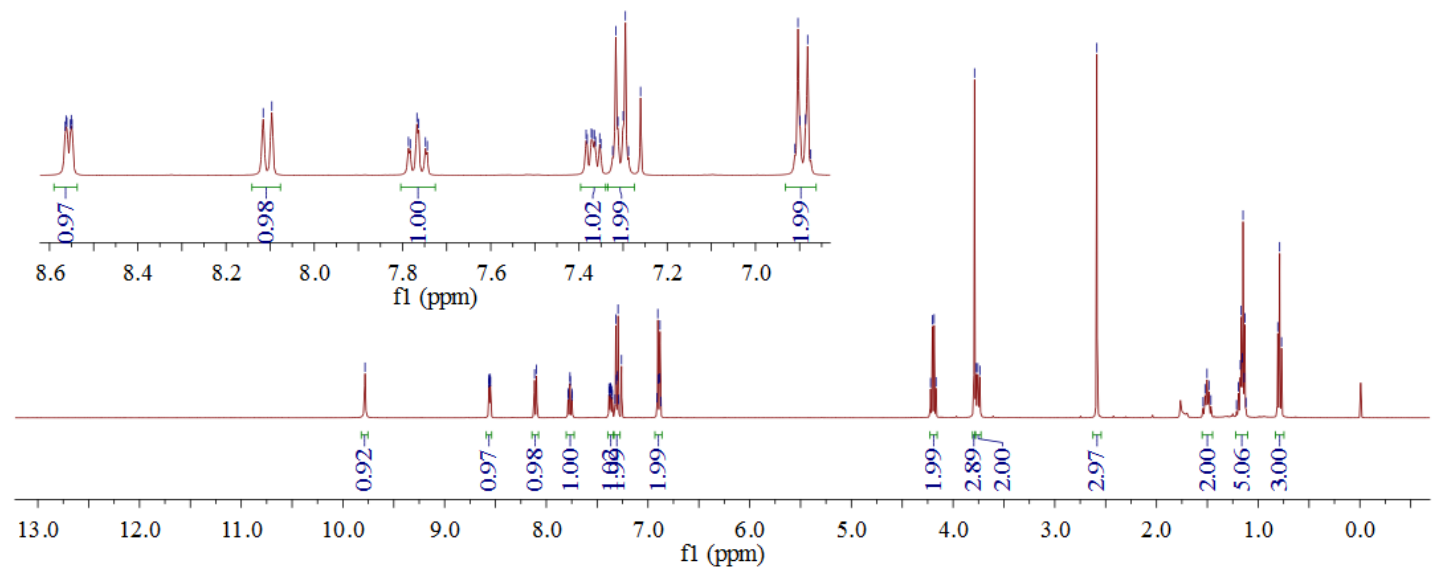



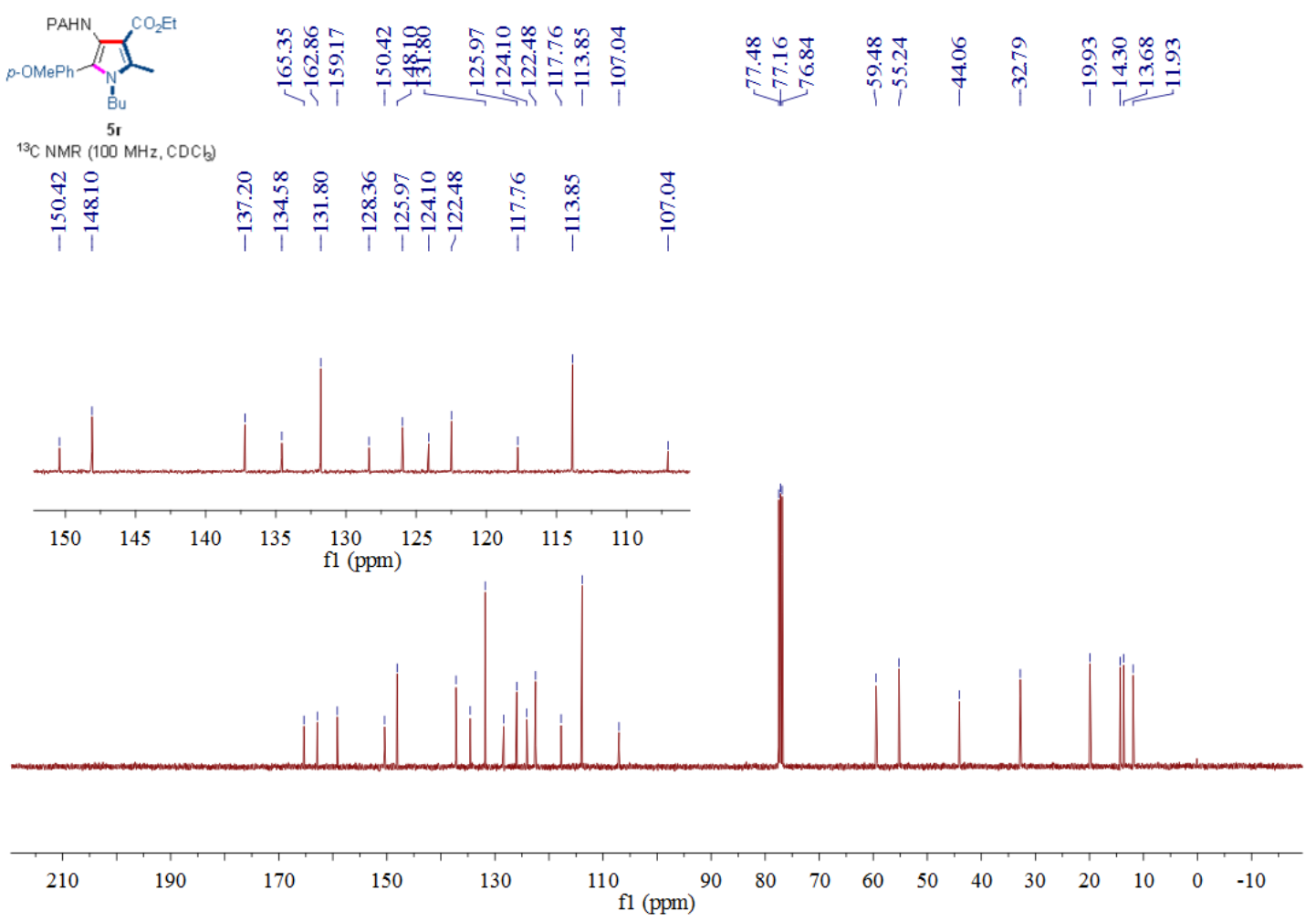

${ }^{1} \mathrm{H}$ NMR and ${ }^{13} \mathrm{C}$ NMR spectra for compound $\mathbf{5 r}$ (using $\mathrm{CDCl}_{3}$ as solvent)
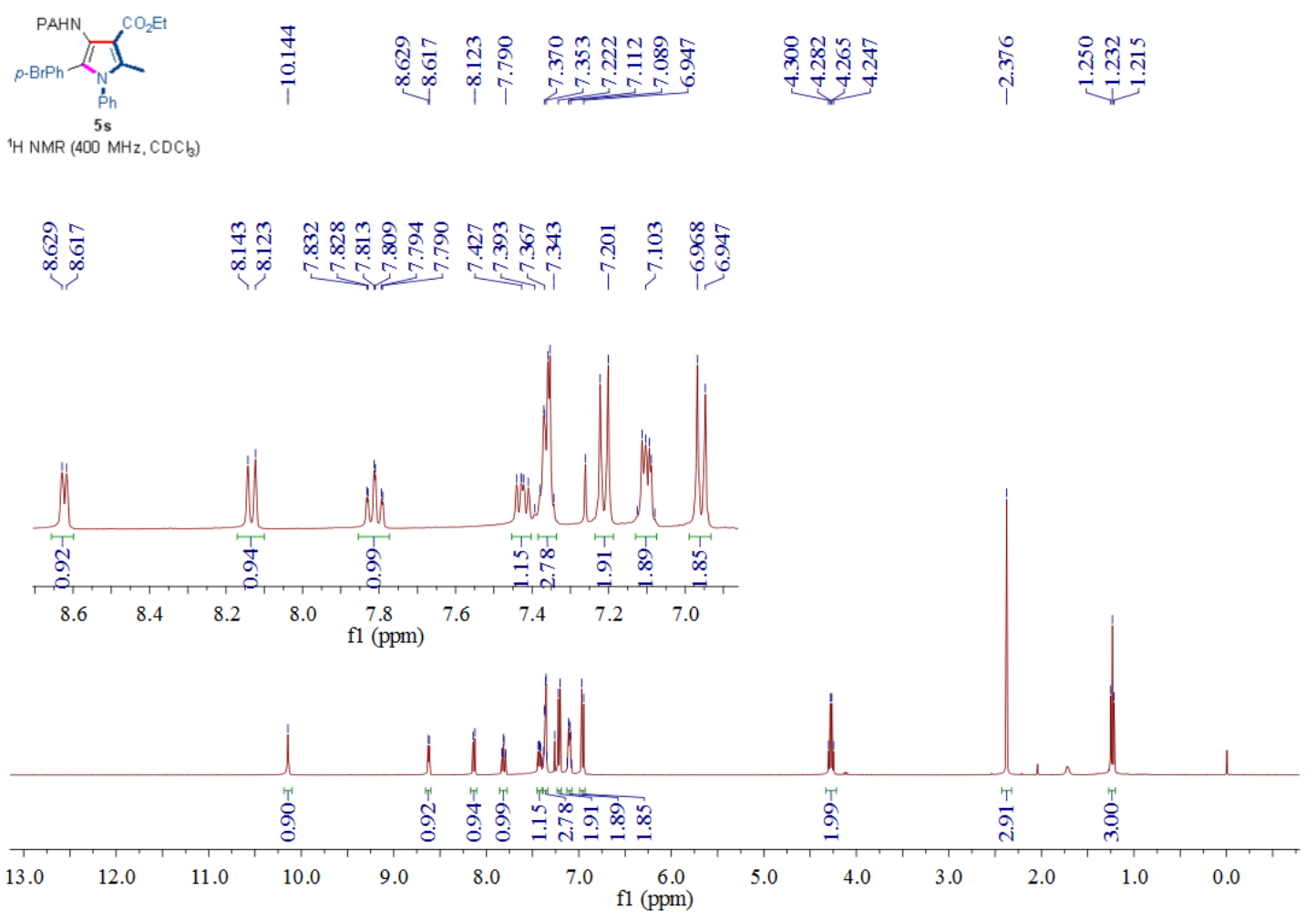


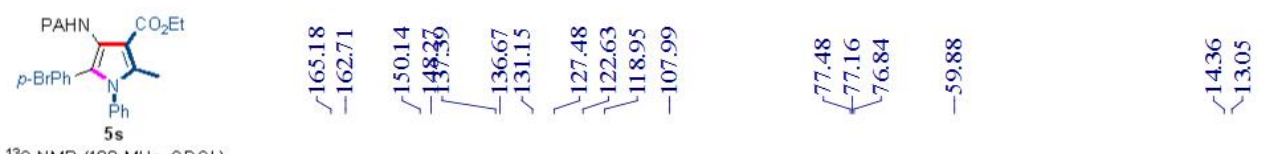

${ }^{13} \mathrm{C} N M R(100 \mathrm{MHz}, \mathrm{CDCb})$

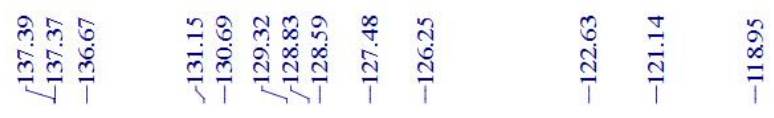

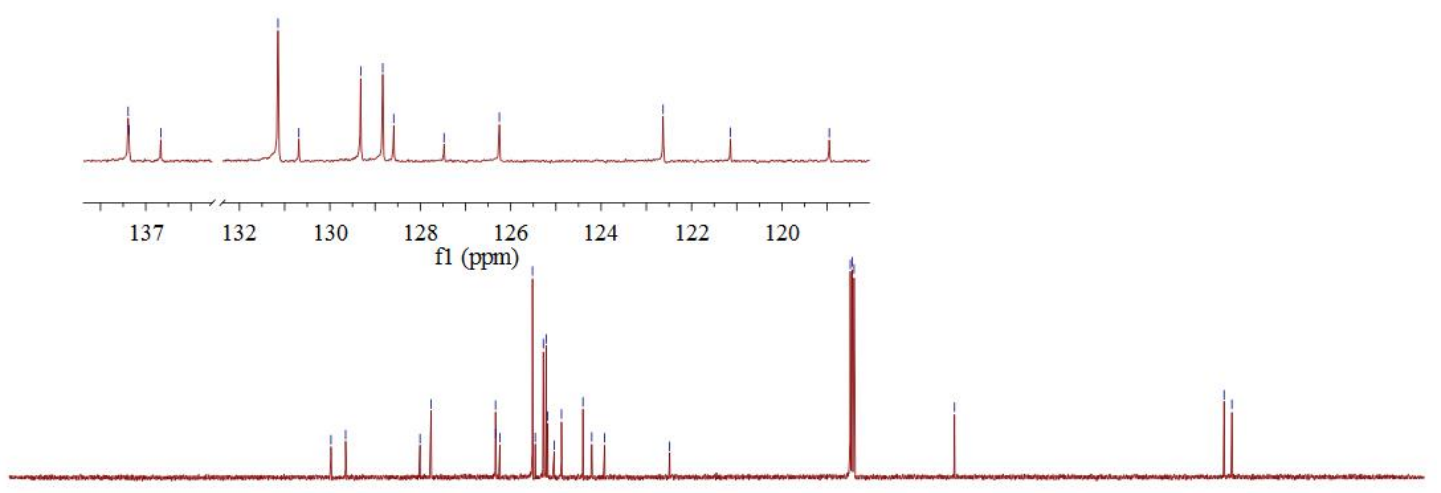

$\begin{array}{lllllllllllllllllll}210 & 190 & 170 & 150 & 130 & 110 & 90 & 80 & 70 & 60 & 50 & 40 & 30 & 20 & 10 & 0 & -10\end{array}$

${ }^{1} \mathrm{H}$ NMR and ${ }^{13} \mathrm{C}$ NMR spectra for compound $\mathbf{5 s}$ (using $\mathrm{CDCl}_{3}$ as solvent)
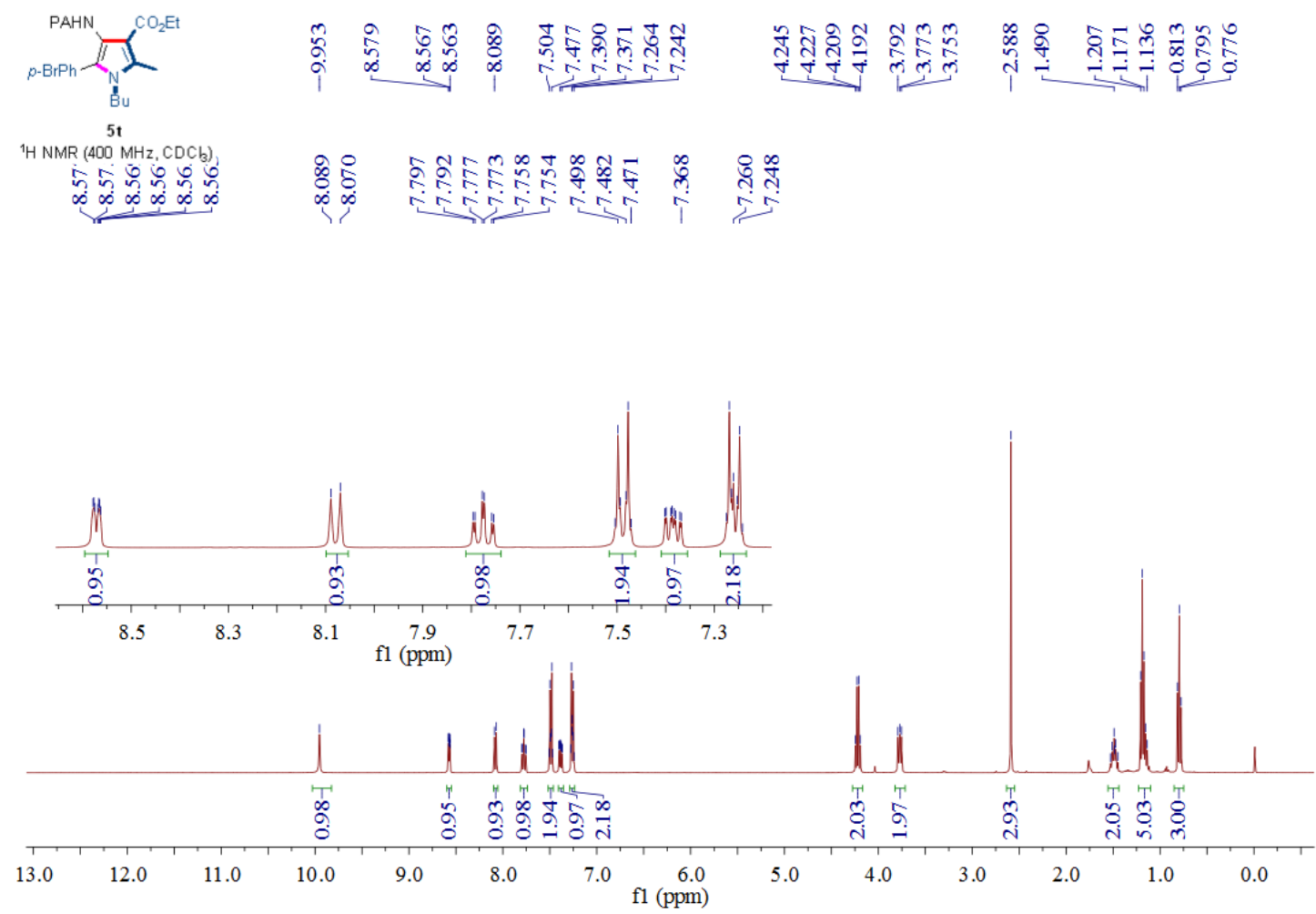


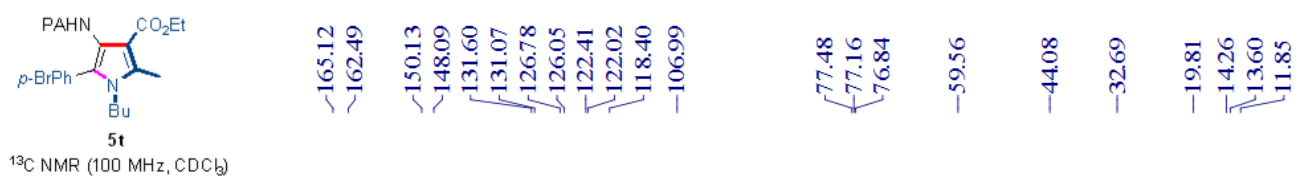

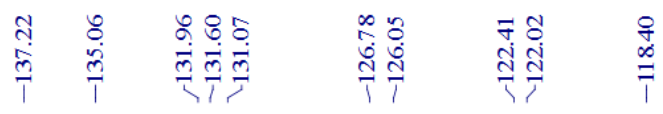
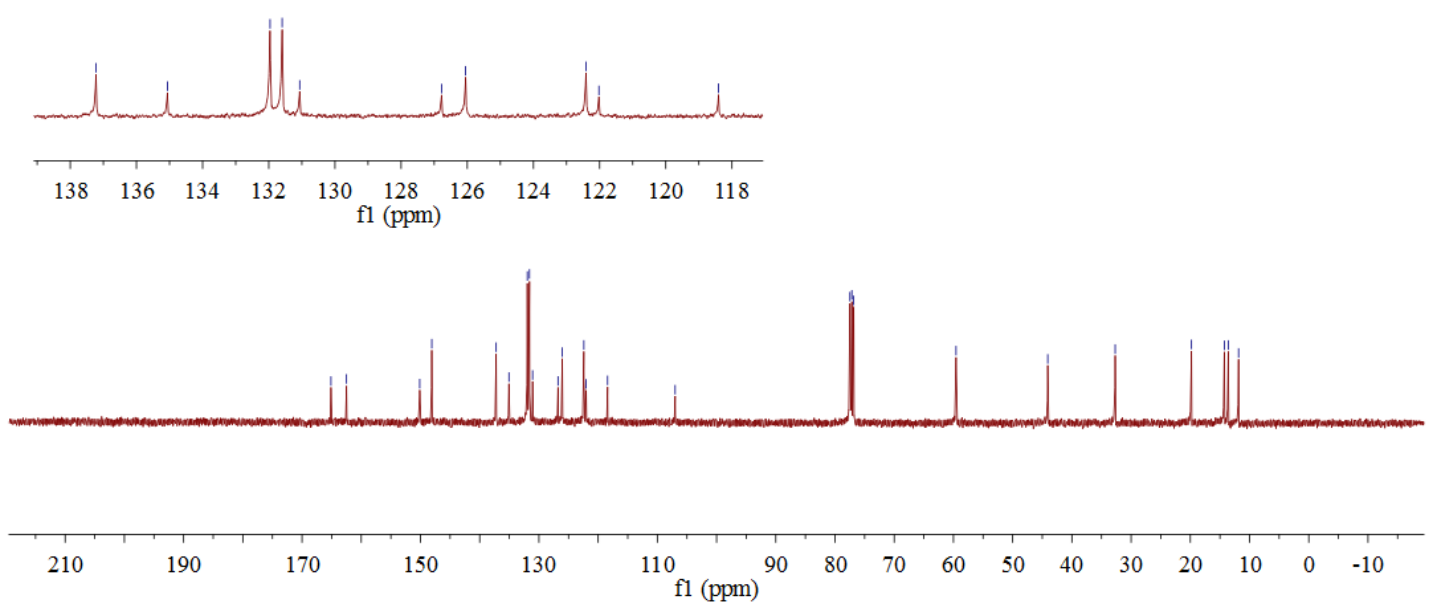

${ }^{1} \mathrm{H}$ NMR and ${ }^{13} \mathrm{C}$ NMR spectra for compound $\mathbf{5 t}$ (using $\mathrm{CDCl}_{3}$ as solvent)
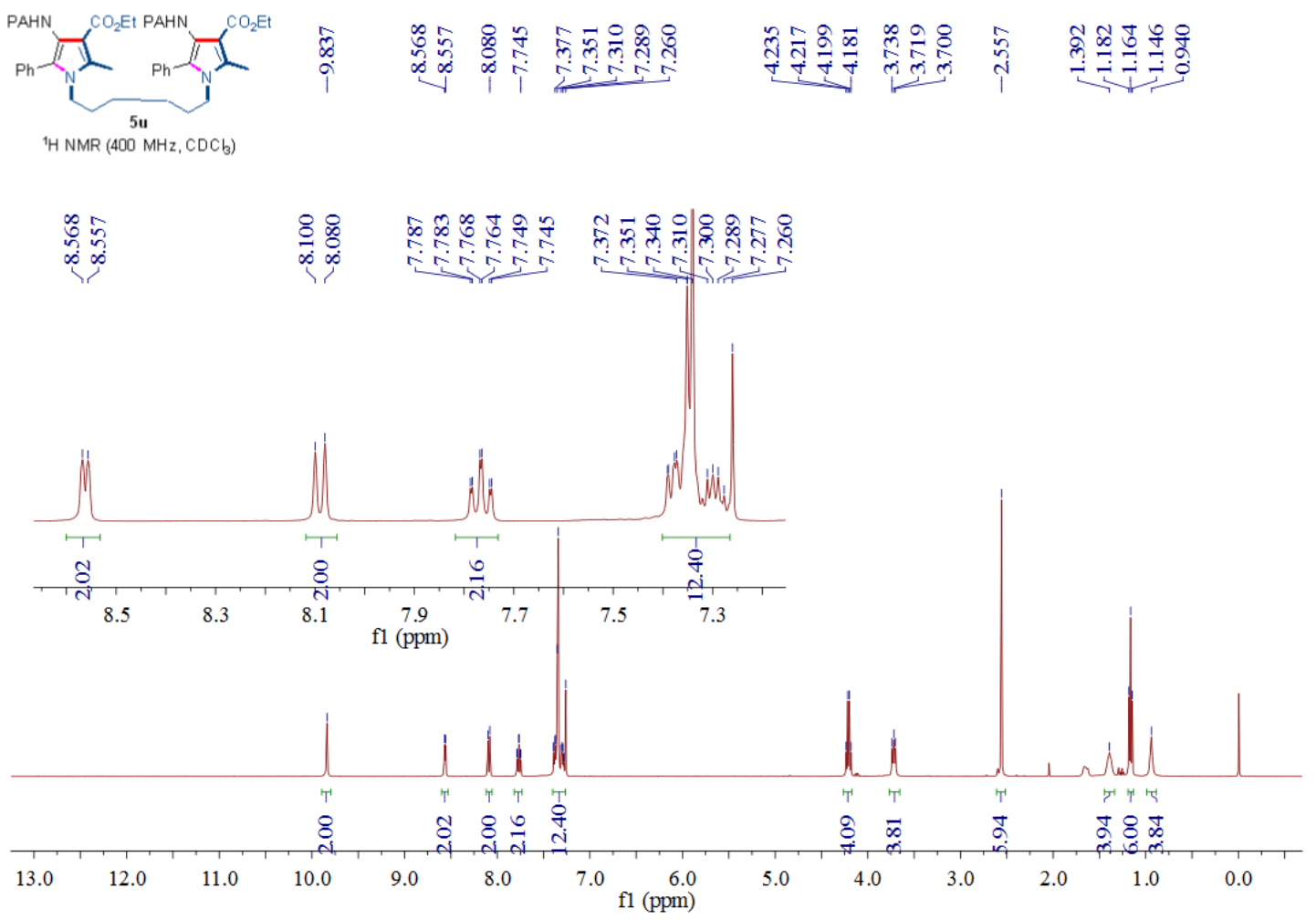


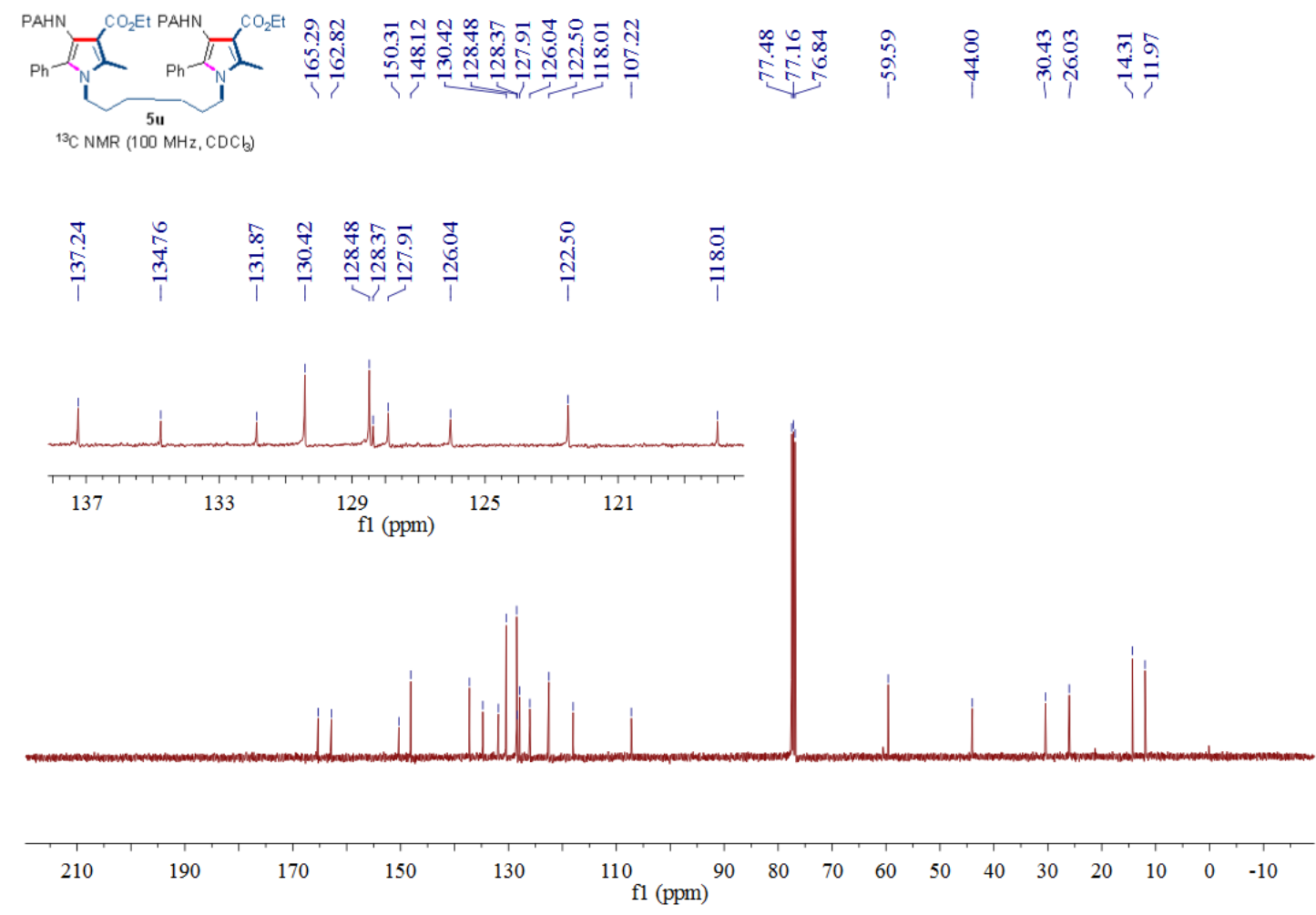

${ }^{1} \mathrm{H}$ NMR and ${ }^{13} \mathrm{C}$ NMR spectra for compound $\mathbf{5 u}$ (using $\mathrm{CDCl}_{3}$ as solvent)

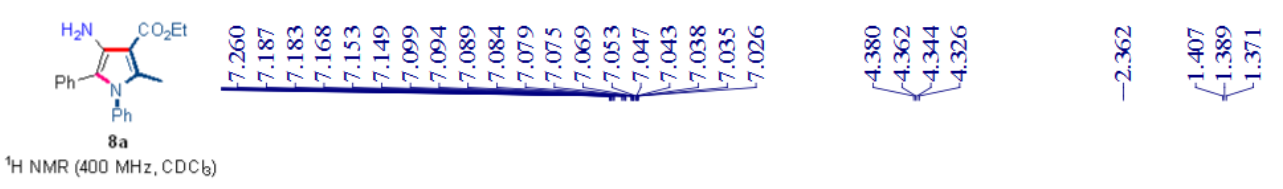

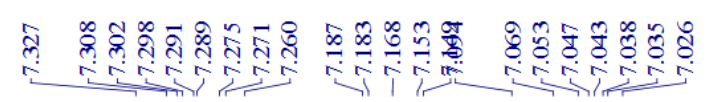
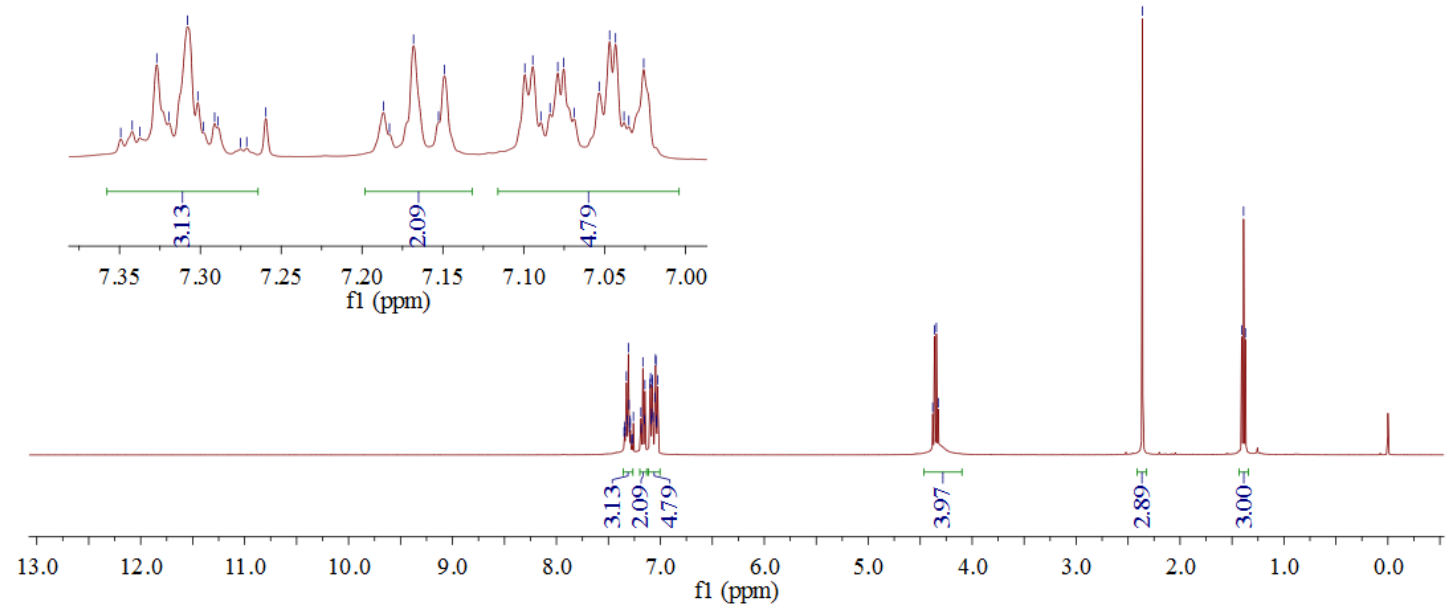

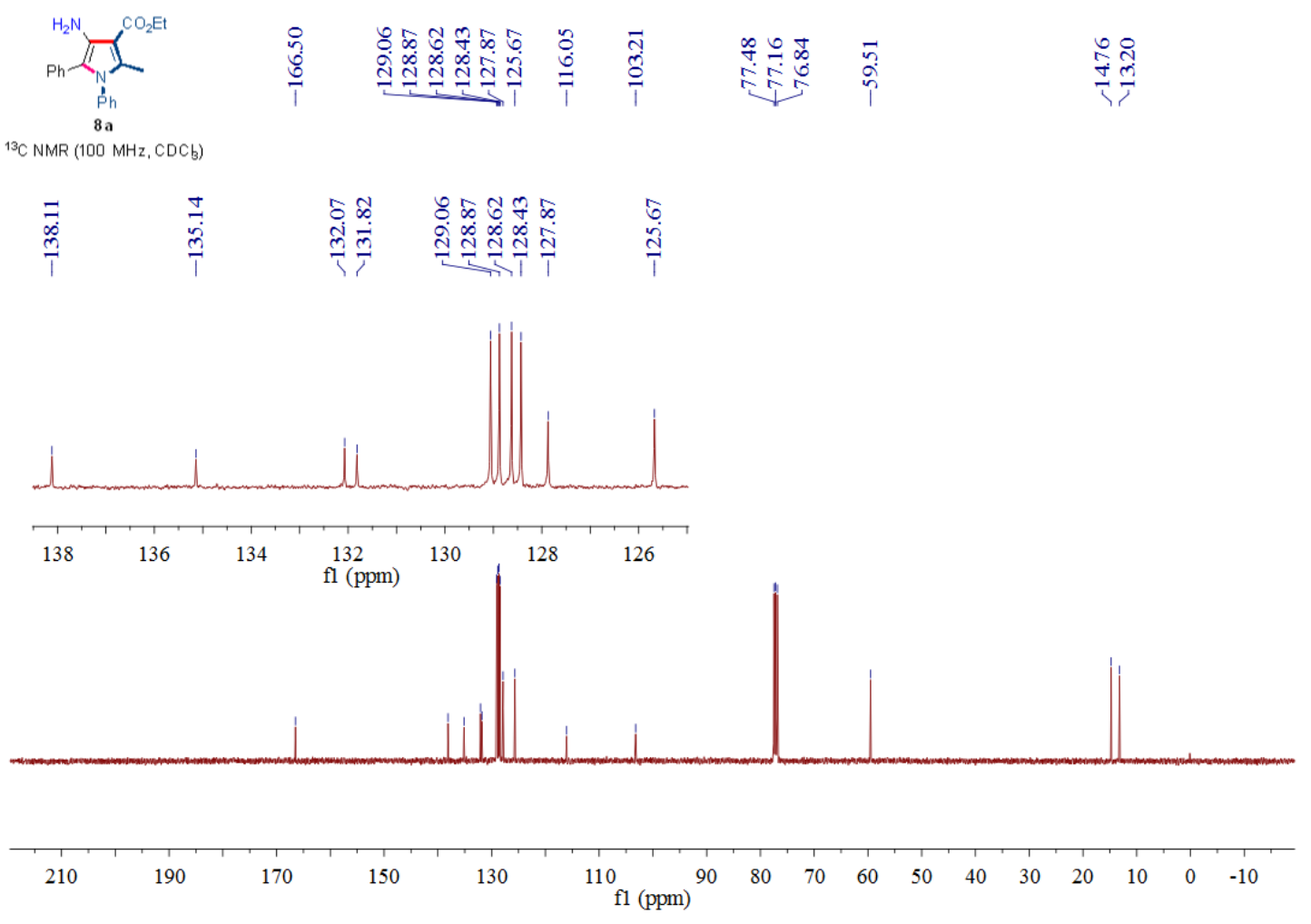

${ }^{1} \mathrm{H}$ NMR and ${ }^{13} \mathrm{C}$ NMR spectra for compound $\mathbf{8 a}$ (using $\mathrm{CDCl}_{3}$ as solvent)
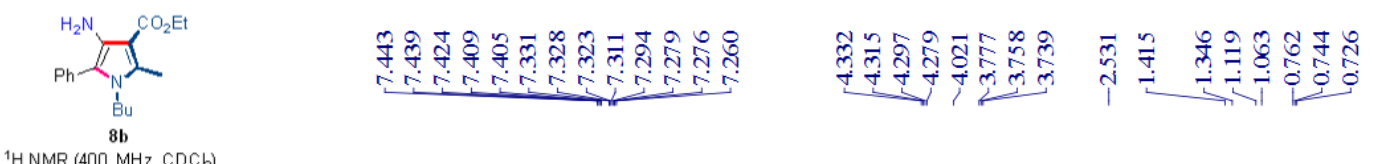

${ }^{1} \mathrm{HNMR}\left(400 \mathrm{MHz}, \mathrm{CDCl}_{3}\right)$

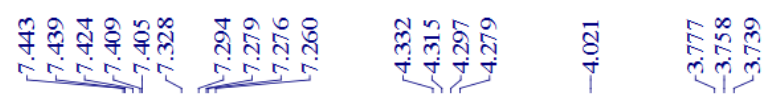
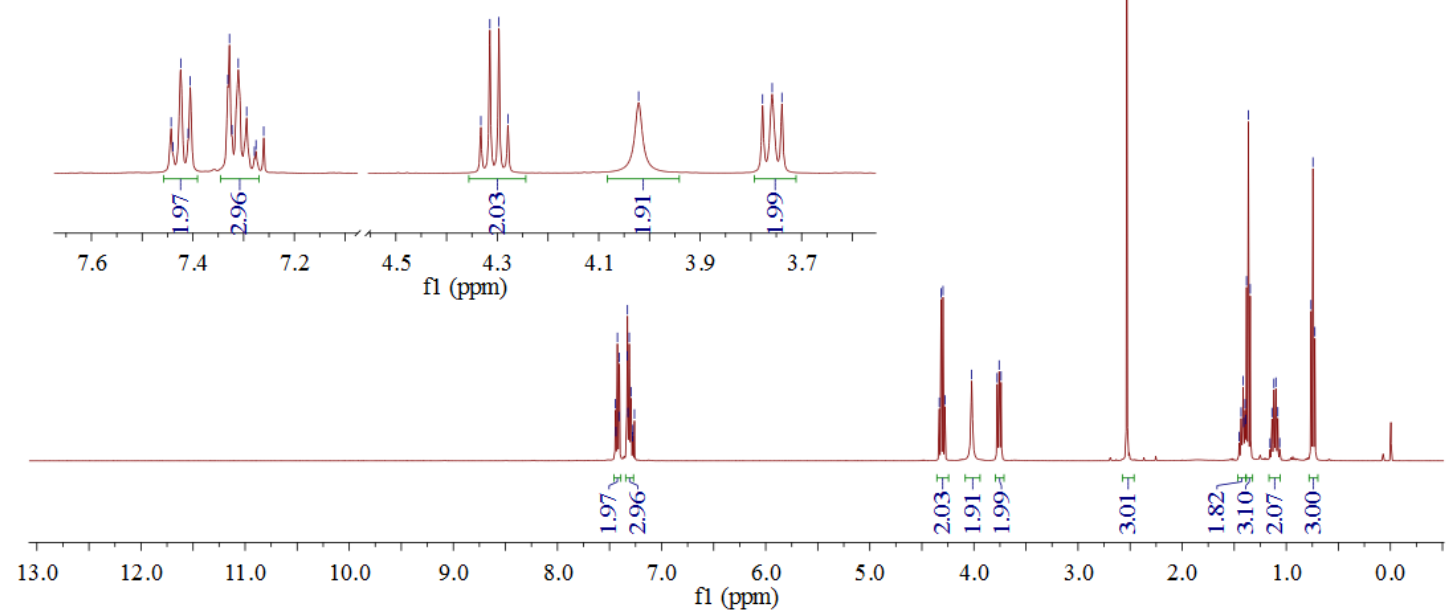

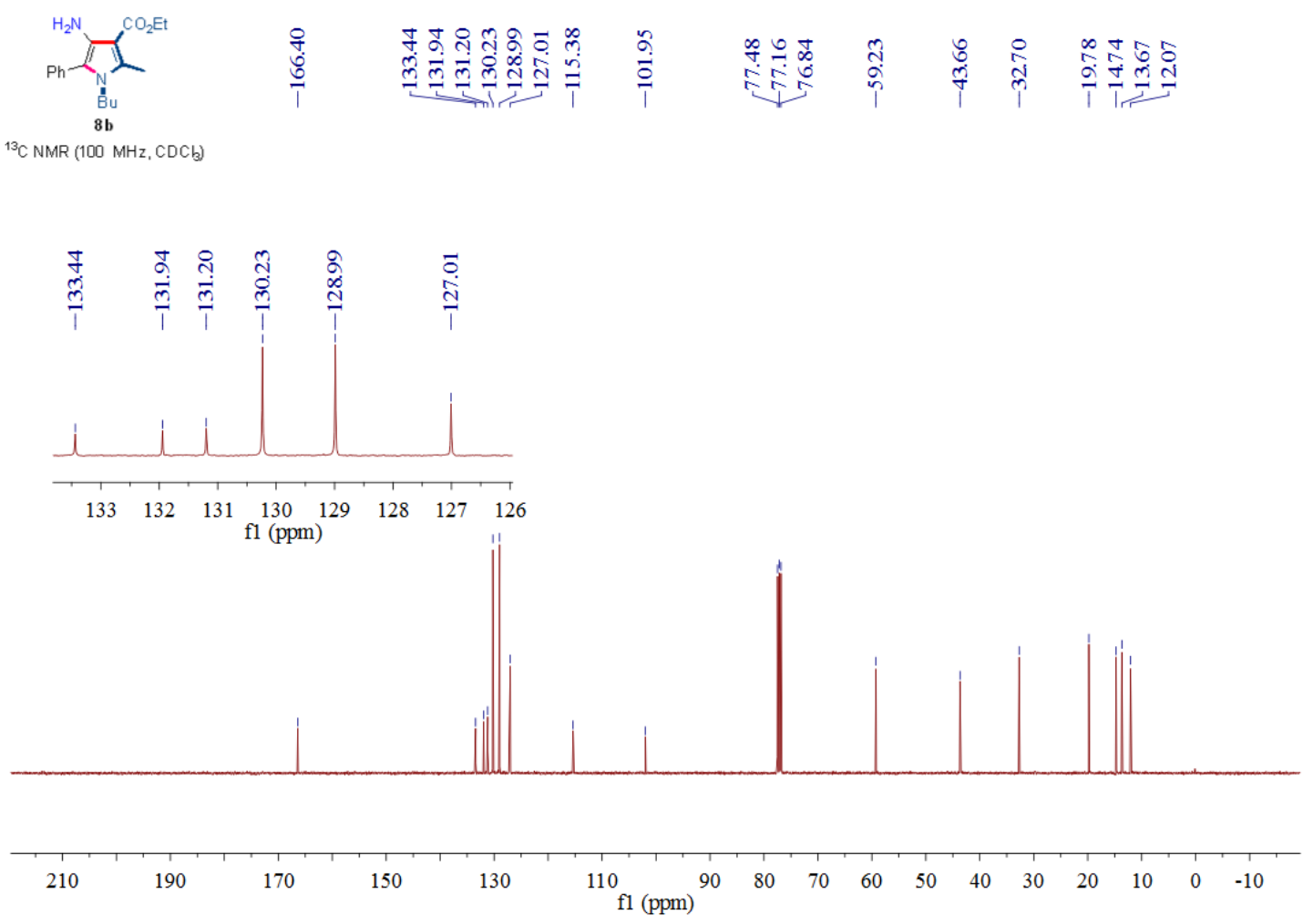

${ }^{1} \mathrm{H}$ NMR and ${ }^{13} \mathrm{C}$ NMR spectra for compound $\mathbf{8 b}$ (using $\mathrm{CDCl}_{3}$ as solvent)

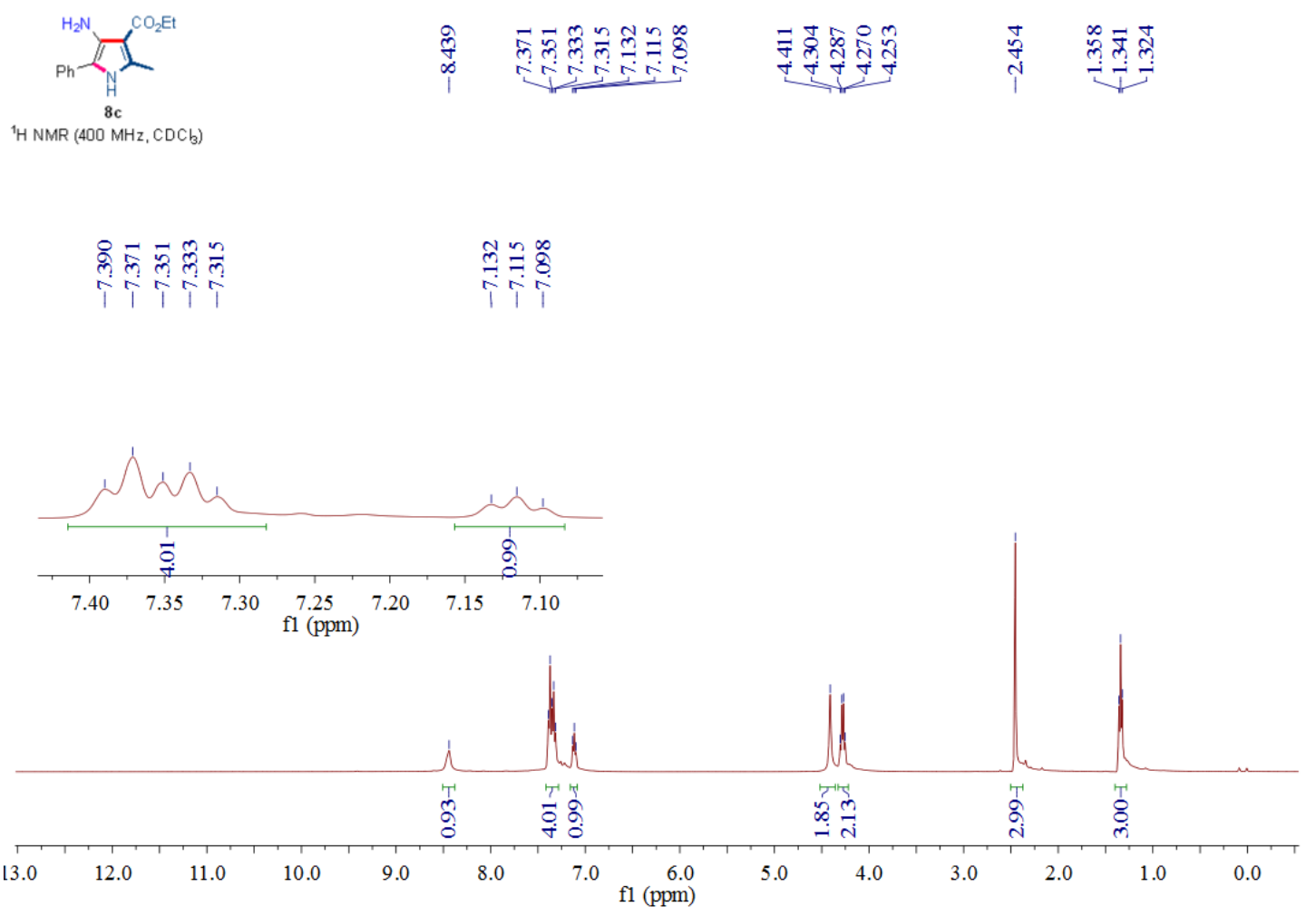




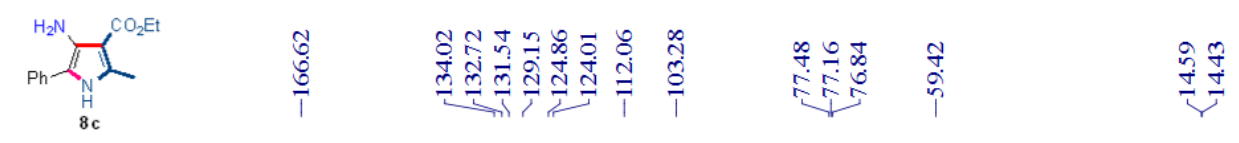

${ }^{13} \mathrm{C}$ NMR $(100 \mathrm{MHz}, \mathrm{CDCl})$

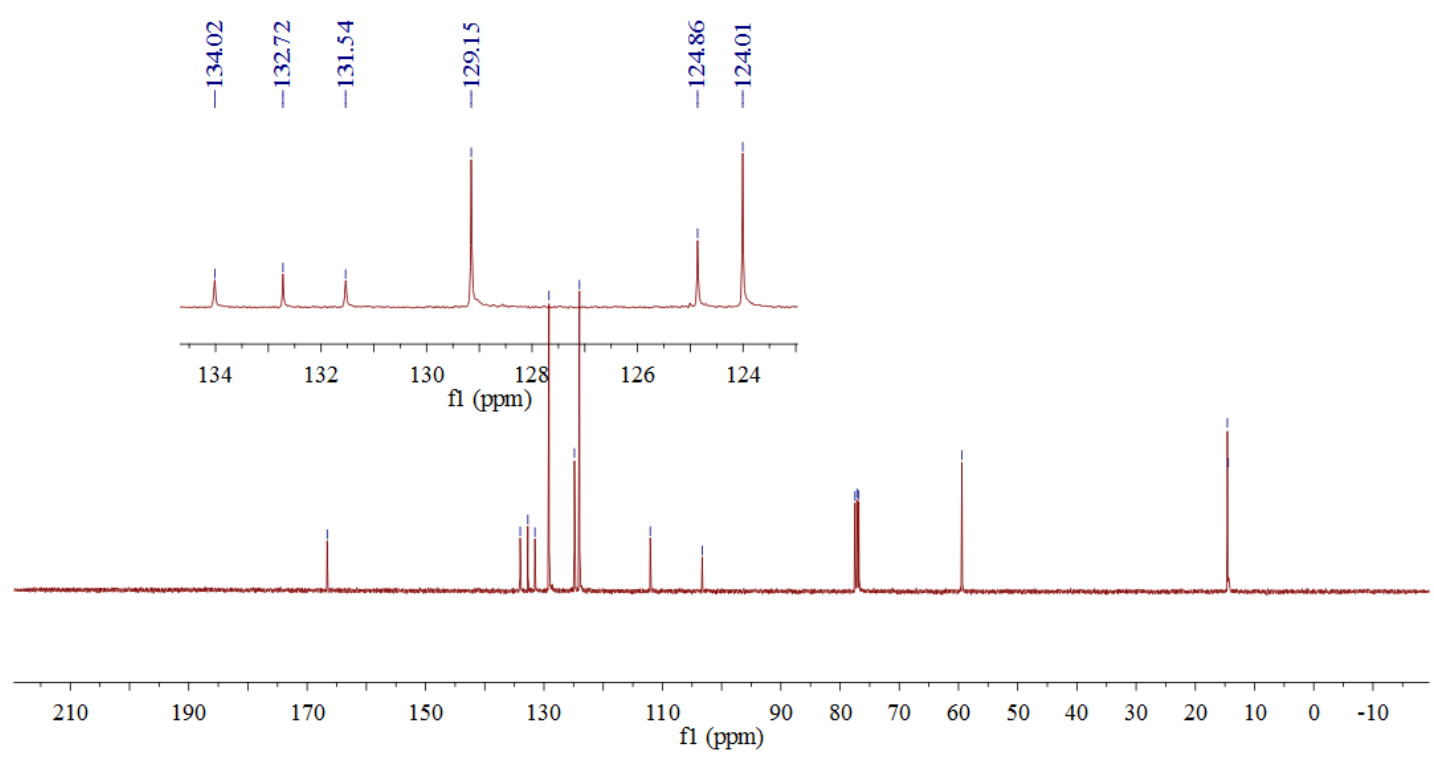

${ }^{1} \mathrm{H}$ NMR and ${ }^{13} \mathrm{C}$ NMR spectra for compound $\mathbf{8 c}$ (using $\mathrm{CDCl}_{3}$ as solvent) 